\title{
FOUR-DIMENSIONAL TOPOLOGY: AN INTRODUCTION
}

\author{
BY RICHARD MANDELBAUM ${ }^{1}$
}

Introduction. This paper is an elaboration of the talk I gave at the American Mathematical Society meeting held at Memphis State University in November, 1977. In that talk I tried to illustrate what was special about four-dimensional topology and mention some of the advances in that subject. I have expanded upon that talk somewhat in this paper in order to give a clearer picture of the geometric ideas and methods currently used in studying four-dimensional manifolds.

The basic problem of geometry is the characterization of spaces by means of algebraic invariants. In its simplest form it is the "Side-Angle-Side" theorem of high school geometry which characterizes triangles up to congruence or the 'Angle-Angle' theorem which characterizes them up to similarity. In the more rarefied realm of algebraic and geometric topology we are especially interested in attaching algebraic objects such as groups, rings, modules, etc., to a space $M$ already known to be a compact connected manifold in such a way that if two manifolds $M, M^{\prime}$ have the same objects associated to them then they are isomorphic (where isomorphic might mean 'homeomorphic' or 'piecewise-linearly homeomorphic' or 'diffeomorphic'). As a result of the undecidability of the word problem for finitely-presented groups [Bo], [BHP] there is, in general, no finite procedure for deciding in all cases whether two groups given by finite sets of generators and relations are isomorphic. Furthermore, as is well known, any finitely-presented group can be realized as the fundamental group of a compact $n$-manifold if $n>4$. Thus provided $n \geqslant 4$ we have no generally effective procedure for determining if $M_{1}^{n}$ is isomorphic to $M_{2}^{n}$. (Henceforth we write ' =' for isomorphic.)

Thus we must ask a more restricted question. In particular we can ask to what extent is $M^{n}$ determined by its homotopy type? We thus consider the following question.

Let $\mathfrak{T}^{n}(X)$ denote the equivalence classes of pairs $(M, f)$, where $M$ is a compact smooth (or PL) $n$-manifold, $f: M \rightarrow X$ is a homotopy equivalence and $(M, f) \sim\left(M^{\prime}, f^{\prime}\right)$ if and only if there is an isomorphism $M \rightarrow M^{\prime}$ making

Received by the editors March 1, 1978.

AMS (MOS) subject classifications (1970). Primary 14J10, 14J25, 32J15, 55A10, 57A15, 57A50, 57C25, 57C35, 57C45, 57D05, 57D10, 57D15, 57D60, 57D65, 57D80, 57E25; Secondary 55A25, 55A35, 57A10, 57D20, 57D40, 57D55.

Key words and phrases. 4-manifold, 4-dimensional $h$-cobordism conjecture, complex surface, almost complete decomposability, framed link, calculus of links, Rohlin's theorem, $\mu$-invariant, surgery, Whitney lemma, flexible handle, torus action, nonlocally flat PL-embedding, handlebody-decomposition.

'Author partially supported by NSF grant MCS 77-04165.

() 1980 American Mathematical Society 0002-9904/80/0000-0001/\$40.75 
the following diagram commute up to homotopy.

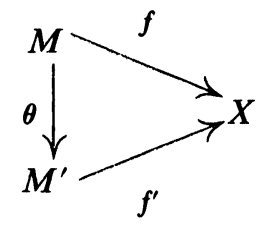

$\mathscr{T}^{n}(X)$ is thus the set of $n$-manifolds in the homotopy type of $X^{\prime}$. We assume $\mathfrak{T}^{n}(X)$ is nonempty and ask what can be said about $\mathfrak{N}^{n}(X)$ ?

If $n=1$ or $n=2$, then $\mathscr{T}^{n}(X)$ will consist of only one element, namely the abstract surface with $g$-handles or with $k$-crosscaps if $n=2$ and the circle $S^{1}$ if $n=1$. In fact if $n=2, \mathfrak{T}^{n}(X)$ is completely determined by its fundamental group $\pi_{1}(X)$, or even more computably by its first homology group $H_{1}(X)$ ! If $n \geqslant 3$ our question becomes much more difficult. To even begin to discuss it we focus our attention on the simpler situation which results if $\pi_{1}(X)=0$.

Thus suppose $\pi_{1}(X)=0$. Then if $n=3$ our question is the classical Poincaré conjecture! Skipping $n=4$ let us check what is known if $n \geqslant 5$.

In this case our problem is the basic simply-connected surgery problem as attacked in [ $\mathbf{B r} \mathbf{1}]$, [N]. Using surgery theory one can analyze the obstructions to deforming homotopy equivalences to isomorphisms. One obtains that for a given cohomology ring and choice of (rational) Pontryagin classes of a manifold $M$ there exists only a finite number of manifolds ring-cohomology equivalent to $M$ and having the same invariants. Furthermore fixing homotopy type and Pontryagin classes again gives only a finite number of isomorphism classes subordinate to the fixed homotopy type.

Summarizing our discussion we arrive at the following diagram (where we include the equivalence relation of $h$-cobordism. Recall that $M^{n}, M^{\prime n}$ are cobordant if there exists a manifold $W^{n+1}$ with $\partial W^{n+1}=M \cup M^{\prime}$. The triple $\left(W^{n+1}, M, M^{\prime}\right)$ is called a cobordism. It is an $h$-cobordism if the inclusion $M \stackrel{i}{\hookrightarrow} W$ is a homotopy equivalence and an $s$-cobordism if $i$ is a simple homotopy equivalence in the sense of Whitehead (see [M 5])).

$$
\begin{aligned}
&\left\{\begin{array}{c}
\text { diffeomorphism } \\
\text { classes of mfds }
\end{array}\right\} \stackrel{\Phi_{1}}{\rightarrow}\left\{\begin{array}{c}
h \text {-cobordism } \\
\text { classes }
\end{array}\right\} \stackrel{\Phi_{2}}{\rightarrow}\left\{\begin{array}{c}
\text { Homotopy type and } \\
\text { Pontryagin classes }
\end{array}\right\} \stackrel{\Phi_{3}}{\rightarrow} \\
&\left\{\begin{array}{c}
\text { Integral cohomology and } \\
\text { Pontryagin classes }
\end{array}\right\}
\end{aligned}
$$

where if $n \geqslant 5$ we have by the $h$-cobordism theorem that $\Phi_{1}$ is 1 -to- 1 , and by the preceding discussion that $\Phi_{2}$ and $\Phi_{3}$ are finite-to-one.

What happens if $n=4$ ? Then all the techniques used to establish the nature of $\Phi_{1}, \Phi_{2}$ and $\Phi_{3}$ above fail. As we shall see in Chapter 1, using entirely different methods one can show that for a simply-connected 4-manifold both $\Phi_{2}$ and $\Phi_{3}$ are in fact 1-to-1! However nothing is known about $\Phi_{1}$ and it may even be infinite-to-one!

It will shortly become apparent that four-dimensional manifolds occupy a peculiar position in the hierarchy of manifolds. On the one hand they are not 
'sufficiently large' to allow one to use the higher-dimensional transversality and general position arguments which have proven so spectacularly successful in high-dimensional topology. Yet on the other hand their dimension is large enough to prevent a straightforward application of more intuitive lowerdimensional methods. This, coupled with the great gaps in our knowledge of three-dimensional topology, makes the subject of 4-manifolds a particularly difficult, but interesting one.

This paper attempts to separate the two approaches. The first chapter establishes some basic theorems about 4-manifolds, while Chapters 2, 3, and parts of 7 explore an approach flavored by lower-dimensional intuition and analogy with lower-dimensional methods. The remaining chapters discuss modifications of higher-dimensional methods rendering them applicable to dimension 4. The paper seeks to be accurate in the statement of theorems, but is not rigorous in their proofs. Since the article originated as a survey talk, proofs of many theorems have been omitted while only geometric ideas and constructions have been sketched for others. Only a basic knowledge of modern topology is assumed, hence the somewhat long introductions and explanations which may be common knowledge to many readers.

This paper could not have been written without the help of many people who influenced me in the course of my study of 4-manifolds. First I owe a great debt of thanks to Boris Moishezon who induced me to study 4-manifolds and with whom I spent many pleasant days studying their properties. I also would like to thank John Morgan, Robert Connelly and Laurent Siebenmann for introducing me to the methods of PL-topology and showing me how topologists think about geometrical problems, as well as Bob Edwards, Cameron Gordon, Jose Montesinos, John Hollingsworth and Andrew Casson for many highly informative (as well as entertaining) conversations explaining various aspects of surgery and knot theory. In addition, thanks are due to Rob Kirby and his students Selman Akbulut, John Harer, Paul Melvin and Steve Kaplan for their invaluable aid in helping me understand the relationship between link theory and 4-manifolds and to Sylvain Cappell and Julius Shaneson for spending much time explaining their own work on 4-manifolds and its relation to higher-dimensional phenomena. In addition Julius Shaneson read the original draft of this manuscript and helped me significantly in producing a hopefully more intelligible final version. Without the help of these mathematicians this paper would never have been written. Of course, I alone am responsible for any inaccuracies in the exposition.

Clearly it is impossible to include all that is known about 4-manifolds in a survey article. I have essentially been guided by my own taste and thus tried to concentrate on areas which were unique from a four-dimensional point of view. I have, however excluded for the most part, results on wildness and taming, such as in [Mt 3], and those on Group actions on 4-manifold such as in ([Or 1], [Or 2], [Pao 1], [Pao 2], [Fin 1], [Fin 2], [FP]). Also I have mentioned only a few results on knottings of $S^{2}$ in $S^{4}$ which has a literature of its own. (See [Lom] or [Fox].)

Notation. By way of notation we will suppose that the basic notions of algebraic topology and differentiable manifolds, as given for example in [ $\mathbf{L g}]$ [Spr 1], are well known to the reader. We will also assume a knowledge of at 
least the elementary properties of piecewise-linear maps and manifolds and topological manifolds.

We recall that a topological (= TOP) manifold $M$ of dimension $n<\infty$ is a metrizable topological space $M$ such that each point $x$ in $M$ admits an open neighborhood $U$ and a homeomorphism $k: U \rightarrow k(U)$ onto an open set of Euclidian space $\mathbf{R}^{n}$.

A piecewise linear $(=\mathrm{PL})$, resp. smooth (DIFF), manifold structure $\Sigma$ on a topological manifold $M$ is a complete (= maximal) piecewise-linearly (PL), resp. smooth (DIFF), compatible atlas of coordinate charts $K$ to $\mathbf{R}^{n}$. PL, resp. DIFF, compatible charts are charts that are related on their overlaps by PL homeomorphisms, resp. diffeomorphisms.

An $H$ manifold where $H=$ PL or DIFF is now defined to be a topological manifold $M$ equipped with an $H$ manifold structure $\Sigma$. The symbol $M_{\Sigma}$ is the usual notation for it; $M$ alone is often used if this is unambiguous, as when $\Sigma$ is in some sense standard or fixed. For example, $\mathbf{R}^{n}, S^{n-1}$ are $H$ manifolds.

Every open subset $M_{0}$ of an $H$ manifold $M$ has an induced $H$ manifold structure; with this structure, $M_{0}$ is called an open $H$ submanifold.

If $\Sigma$ is an $H$ structure on $M$ and $h: M \rightarrow N$ is a homeomorphism, the image $H$ structure $h \Sigma$ on $N$ has as a typical chart a composed homeomorphism

$$
h U^{h-1} \rightarrow \stackrel{k}{\rightarrow} k U
$$

where $k: U \rightarrow k U$ is a chart of $\Sigma$.

A homeomorphism of $H$ manifolds $h: M_{\Sigma} \rightarrow N_{\Sigma^{\prime}}$ is an $H$ isomorphism if $\Sigma^{\prime}=h \Sigma$, or equivalently $\Sigma=h^{-1} \Sigma^{\prime}$. We then write $M_{\Sigma} \cong N_{\Sigma^{\prime}}$; and in case $N_{\Sigma^{\prime}}$ is in addition an open submanifold of an $H$ manifold $N^{+}$, we call the composition $h_{+}: M_{\Sigma} \rightarrow N^{+}$of $h$ with the inclusion $N_{\Sigma^{\prime}} \hookrightarrow N^{+}$an $H$ imbedding.

One can define an $H$ map $f: M \rightarrow N$ of $H$ manifolds to be a map of the underlying sets which, when expressed locally in terms of coordin $t$ chart is, respectively, continuous if $H=\mathrm{TOP}$, piecewise-linear if $H=\mathrm{PL}$, or differentiable $C^{\infty}$ if $H=$ DIFF. There results a category of $H$ manifolds which is occasionally itself denoted $\boldsymbol{H}$. Note that its isomorphisms are as described above. The sign $\cong$ indicates isomorphism in whatever category we happen to be working.

A PL map that is a homeomorphism is a PL isomorphism. This is clearly quite untrue for DIFF in place of PL, as the map $x \rightarrow x^{3}$ on $R^{1}$ shows.

A space $X$ is said to be triangulated if it is a simplicial complex; the term is particularly convenient if space carries additional structure such as a DIFF (manifold) structure.

A simplicial complex PL isomorphic to a PL manifold is known as a combinatorial manifold; it is characterized by the fact that the link of each simplex is PL isomorphic to a PL sphere or ball.

As we will point out later not every triangulation is combinatorial.

A map $f: X \rightarrow Y$ in any category $H$ we have met (from the category of topological spaces and continuous maps on) is called an $H$ trivial bundle if there exists an $H$ object $F$ and an $H$ isomorphism $\theta: F \times Y \rightarrow X$ such that $f \theta=p_{2}$ where $p_{2}$ is the projection to $Y$. More generally, $f$ is called an $H$ 
(locally trivial) bundle or bundle projection if each point $y$ in $Y$ has an open neighborhood over which $f$ is a $\boldsymbol{H}$ trivial bundle.

We can similarly define $H$ structures on manifolds with boundary by replacing $\mathbf{R}^{n}$ by $\mathbf{R}_{+}^{n}$ (= Euclidian half-space) in all our definitions above. This leads to some problems in the smooth case (one must consider manifolds with corners) which we ignore (see [KS 1] for an elegant treatment). Note that products always exist in $H$ (provided we amend DIFF to allow the possibility of corners, as noted above).

We define a homotopy as a map $F: I \times X \rightarrow I \times Y$ such that $p_{1}=p_{1} F$, where $p_{1}$ denotes projection to the first factor $I$. In case $F$ is an isomorphism it is called an isotopy. When $F$ is an open imbedding it is called an isotopy through open imbeddings.

This language applies in the several categories $H$ we have met, not just the category of continuous maps of topological spaces.

Note that $F$ is uniquely determined by the family $f_{t}: X \rightarrow Y, 0<t<1$, of maps such that $F(t, x)=\left(t, f_{t}(x)\right)$ for all $(t, x)$ in $I \times X$. We often write $f_{t}$, $0<t<1$, instead of $F$; and we often say that $F$ is a homotopy from $f_{0}$ to $f_{1}$, writing $f_{0} \simeq f_{1}$. Note that $f_{t}$ will inherit from $F$ the property of being an $H$ isomorphism or an $H$ open imbedding.

An $H$ concordance $\Gamma$ is an $H$ structure on a product $I \times M$ where $M$ is a TOP manifold, so that $\Gamma$ gives by restriction $H$ structures $\Sigma_{i} \times\{i\}$ on $M \times\{i\}$ for $i=0,1$; and we say that it gives an $H$ concordance $\Gamma: \Sigma_{0} \Omega \Sigma_{1}$.

Other than the standard notation on manifolds we use the notation $P$ घ $Q$ to denote the boundary-connected sum of the manifolds (with boundary) $P, Q$ and $P \# Q$ their interior connected sum. (See [RIf] or [KM 2] for definitions.)

We will have occasion to discuss isometries of inner-product spaces. We recall following $[\mathbf{M H}]$ that an inner-product space is a finitely generated projective module $G$ over a commutative ring with unit $R$ posessing a nondegenerate bilinear form $\lambda: G \times G \rightarrow R$. For our purposes such modules will always be assumed to be free and therefore the form $\lambda$ will be representable by a matrix $A_{\lambda}$ relative to any choice of basis of $G$. The nondegeneracy of $\lambda$ will then be equivalent to assuming some (and thus all) $A_{\lambda}$ has $\operatorname{det}\left(A_{\lambda}\right)$ a unit in $R$. The symmetry or skew-symmetry of $\lambda$ will be defined in the obvious fashion.

If $(G, \lambda),\left(G^{\prime}, \lambda^{\prime}\right)$ are inner-product spaces then an isomorphism between them will mean a module-isomorphism $\phi: G \rightarrow G^{\prime}$ satisfying $\lambda^{\prime} \circ(\phi \times \phi)=$ $\lambda$. This will sometimes also be called an isometry or in the case of automorphisms of $(G, \lambda)$ simply an automorphism of the form $\lambda$.

We can define orthogonal direct sums, annihilators, etc., in the obvious fashion and we use such concepts when necessary. A particularly significant invariant of a symmetric inner-product space $(G, \lambda)$ over $\mathbf{Z}$ is the signature $\sigma(G, \lambda)$ of $(G, \lambda)$. This is defined as the number of positive terms minus the number of negative terms in any diagonalization of $A_{\lambda}$ over $\mathbf{Q}$ and can be shown to be an invariant of $(G, \lambda)$. (See [MH], [Hirz 2].) (One can similarly define the signature of a Hermitian form in a similar fashion using the well-known diagonalization theorem for such forms. See [MS].) The most significant inner-product space we will encounter is the one obtained by restricting the cup-product form $\bar{L}_{M}$ of a compact orientable $4 k$-dimensional 
manifold $M^{4 k}$ to the symmetric bilinear form:

$$
L_{M}: H^{2 k}(M ; \mathbf{Z}) / \text { Torsion } \times H^{2 k}(M ; \mathbf{Z}) / \text { Torsion } \rightarrow \mathbf{Z} \approx H^{4 k}(M ; \mathbf{Z}) \text {. }
$$

We will discuss the properties of $L_{M}$ rather thoroughly (at least if $k=1$ ) in Chapter 1 and merely note at this point that one defines the index or signature $\sigma(M)$ of $M$ as the signature $\sigma\left(H^{2 k}(M), L_{M}\right)$ of $L_{M}$.

Closely related to the concept of an inner product space is that of a quadratic inner-product space.

We say $\mu: G \rightarrow R$ is a quadratic form if $\mu(\alpha x)=\alpha^{2} \mu(x)$ and $\mu(x+y)-$ $\mu(x)-\mu(y)=x \cdot y$ defines a bilinear form - on $G$. Clearly if $\lambda$ is a bilinear form on $G$ then $q(x)=\lambda(x, x)$ is a quadratic form on $G$. We say $(G, M)$ is a quadratic inner-product space if the bilinear form $\cdot$ on $G \times G$ defined by $M$ is nondegenerate. Notice that any inner-product space $(G, \lambda)$ has an associated quadratic form $M$ such that $x \cdot y=\lambda(x, y)+\lambda(y, x)$. One then immediately sees that if 2 is a unit in $R$ then symmetric bilinear forms correspond bijectively with quadratic forms (up to units). Furthermore as long as $\mathbf{2}$ is not a zero-divisor in $\boldsymbol{R}$ then any symmetric bilinear form $\lambda$ satisfying $\lambda(x, x) \equiv 0 \bmod 2 R$ arises from a unique quadratic form.

We shall pay particular attention to inner-product spaces over $\mathbf{Z}$ and over $\mathbf{Z}_{2}$. As 2 is not a zero-divisor in $\mathbf{Z}$ we shall generally only speak about $\mathbf{Z}$-bilinear forms with the associated quadratic form being understood to be the canonical one. For spaces over $\mathbf{Z}_{2}$ we shall generally specify both quadratic and bilinear forms and their relationship. In Chapter 1 we shall discuss the classification of inner-product spaces and their relationship to the theory of 4-manifolds. Generally rather than speak about classifying the space $(G, \lambda)$ we shall refer to classifications of the form $\lambda$.

In Chapter 5 we shall also speak about Hermitian forms on $G$. If $R$ has an involution $\alpha \rightarrow \bar{\alpha}$ then a Hermitian form on $G$ will be a form $\lambda: G \times G \rightarrow R$ satisfying $\lambda(y, x)=\overline{\lambda(x, y)}$ and linear in the first variable. Note that $\lambda(\alpha x, \beta y)=\alpha \lambda(x, y) \bar{\beta}$. $\lambda$ will be called nondegenerate if the Hermitian matrix corresponding to it is nonsingular.

In the course of our article we will have occasion to discuss cobordisms and bordism groups. The definition of these concepts will be recalled in Chapter 1. It would take us too far afield to go through a complete definition of simple homotopy equivalence and Whitehead groups $\mathrm{Wh}(\Lambda)$, and for these we refer the reader to [M 5]. Since most of the examples we give will have fundamental groups $\pi$ with $\mathrm{Wh}(\pi)=0$ the reader will not suffer if he regards all homotopy equivalences as simple in any example he encounters. We shall consider the definition and properties of vector bundles and their characteristic classes (Pontryagin, Steifel-Whitney and Chern) to be part of the subject matter of elementary algebraic topology (see [Spr 1] for example) and thus known to the reader. (See [Hus], or [MS] for a very pretty and straightforward exposition.) We will therefore use without comment the notion of classifying spaces $B_{\mathrm{O}(n)}, B_{\mathrm{SO}(n)}$ for smooth, resp. smooth oriented, vector bundles and the bijective correspondence between $\operatorname{Vect}_{n}(X)$ and $\left[X, B_{\mathrm{O}(n)}\right]$ where $\operatorname{Vect}_{n}(x)$ are the $n$-plane bundles over the CW-complex $X$ and $[X, Y]$ denotes the homotopy classes of maps of $X$ into $Y$. [There is a corresponding bijection for smooth oriented vector bundles as well.] We shall discuss in Chapters 6 and 7 
the corresponding bijections for the piecewise-linear and topological categories. We note in this regard that DIFF, PL and TOP will stand for the categories of smooth manifolds and smooth maps, PL manifolds and PL maps, and topological manifolds and topological maps; $B_{\mathrm{TOP}(n)}$, resp. $B_{\mathrm{PL}(n)}$, will then refer to the classifying spaces for the topological, resp. PL, analogue of $n$-plane bundles. [We recall that the notation $B_{\mathrm{O}(n)}$ comes from the homotopy equivalence between $\operatorname{DIFF}(n)=$ \{smooth origin-preserving diffeomorphisms of $\left.\mathbf{R}^{n}\right\}$ and $\mathrm{O}(n)=\{n$-dimensional orthogonal group $\}$.]

We will also use some elementary facts about stable vector bundles and their PL and TOP analogues. Here [Hus] is a good reference for the smooth case with the PL and TOP categories behaving similarly. Whenever we refer to a stable bundle over an $n$-dimensional manifold $X^{n}$ the reader can simply think of a $k$-plane bundle $\xi$ over $X$ with $k \gg n$. Stable bundles have classifying spaces which we write as $B_{\mathrm{O}}, B_{\mathrm{PL}}, B_{\mathrm{TOP}}$ but here again the corresponding $B_{\mathrm{O}(k)}, B_{\mathrm{PL}(k)}, B_{\mathrm{TOP}(k)}$ serve as well if $k$ is sufficiently large. (We note for the convenience of the reader that if $\xi^{k}$ and $\xi^{l}$ are a $k$-plane and an $l$-plane bundle over $X$ then they are stably equivalent if there exist trivial bundles $\varepsilon^{m-k}, \varepsilon^{m-l}$ over $X$ of fiber dimension $m-k \geqslant 0$ and $m-l \geqslant 0$ such that $\xi^{k} \oplus \varepsilon^{m-k}$ is isomorphic to $\xi^{l} \oplus \varepsilon^{m-l}$.)

For the convenience of the reader we also recall that if $X$ is a manifold then the characteristic classes of $X$ always refer to the characteristic classes of the tangent bundle $\mathcal{T}_{X}$. Then we have, writing $w_{i}(X), c_{i}(X), p_{i}(X)$ for the Steifel-Whitney, Chern, Pontryagin classes, respectively, of $X$ that:

(1) $X$ is orientable iff $w_{1}(X)=0$.

We can always interpret $w_{1}(X)$ as an element in $\operatorname{Hom}_{\mathbf{z}_{2}}\left(\pi_{1}(X), \mathbf{Z}_{2}\right)$ sending loops preserving orientation to +1 and those reversing orientation to -1 $\left(\mathbf{Z}_{2}=\{ \pm 1\}\right)$.

(2) We say $X$ is a spin manifold if $w_{2}(X)=0 . w_{1}(X)$ is the obstruction to orienting the tangent bundle $\mathscr{T}_{X}$ of $X$ while $w_{2}(X)$ is the obstruction to putting a spin structure on $\mathscr{T}_{X}$, i.e. lifting

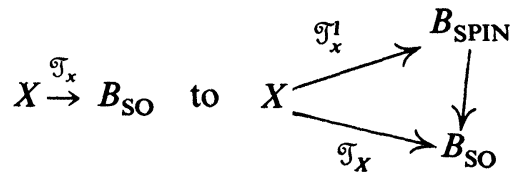

If $X$ is a 4-manifold then $w_{2}(X)$ is the unique class in $H^{2}\left(X ; \mathbf{Z}_{2}\right)$ such that $w_{2}(X) \cup Y=Y \cup Y$ for all $Y \in H^{2}\left(X ; Z_{2}\right)$.

(3) If $C_{1}[X]_{\bmod 2}$ is a mod 2 reduction of $c_{1}[X] \in H^{2}\left(X ; \mathbf{Z}_{2}\right)$ to a class in $H^{2}\left(X ; \mathbf{Z}_{2}\right)$ then $c_{1}[X]_{\bmod 2}=w_{2}[X]$. One also has that under the isomorphism $H^{4}(X ; \mathbf{Z}) \approx \mathbf{Z}$, for almost complex manifolds $X$ we have $c_{2}[X]$ goes to $\chi[X]=\Sigma_{0}^{4}(-1)^{i}$ rk $H^{i}(X ; \mathbf{Z})$ and under $H^{4}\left(X ; \mathbf{Z}_{2}\right) \approx \mathbf{Z}_{2}, \quad w_{4}[X]=c_{2}[X]$ $(\bmod 2)($ if $X$ is almost complex $=\chi[X] \bmod 2)$.

(4) For oriented 4-manifolds $p_{1}[X]=3 \sigma(X), \sigma(X)$ the signature of the cup-product pairing on $X$, and by [Frk], $w_{2}[X]$ is always the reduction of an integral class so that its dual homology class is always representable by an oriented submanifold.

We shall also consider the Poincare duality theorem for orientable mani- 
folds with $\mathbf{Z}$-coefficients and all manifolds with $\mathbf{Z}_{2}$-coefficients as being known to the reader. (See [Spr 1], [MS], or [Br 1].)

In Chapter 5 we shall consider Poincaré complexes. The reader can either replace the word 'Poincare complex' by 'topological manifold' wherever he finds it or refers to [Br 2], [Wa 3] for definitions. We shall sometimes also speak of twisted Z-coefficients in the context of duality or isomorphism theorems. Here again the reader may either substitute $\mathbf{Z}$, resp. $\mathbf{Z}_{2}$, for twisted $\mathbf{Z}$ in the orientable, resp. nonorientable, case or refer to [Wa 3] for definitions.

Lastly we will on occasion make use of transversality, general position and local-flatness arguments. The full statement of the transversality theorem we use can be found in Chapter 5. However, intuitive concepts of transversality and general position will usually suffice to follow our arguments.

We shall say that a proper PL or TOP embedding $M^{m} \hookrightarrow N^{m+q}$ is locally flat if each point $p \in M$ has a neighborhood $U$ in $N^{m+q}$ such that $(N, M) \cap$ $U$ is isomorphic to $D^{m} \times D^{q}, D^{m} \times 0$. Note that smooth embeddings are clearly always locally flat [Lg] while any embedding is locally flat if $q \geqslant 3$ [RS, Chapter 7].

If $P$ and $Q$ are submanifolds of a given manifold $M$ we will use the notation $P \pitchfork Q$ to mean that $P$ intersects $Q$ transversely. Note that $P \pitchfork Q$ must then always be a submanifold of $M$.

The specific contents of our paper can be elucidated from the following table of contents.

\section{TABLE OF CONTENTS}

Chapter 1. The basic structure of 4-manifolds

1.1 The theorems of Whitehead and Wall

1.2 Four manifolds and symmetric forms

1.3 Almost complete decomposability and analytic surfaces

1.4 Rohlin's theorem

1.5 Aspherical 4-manifolds

Chapter 2. Complex surfaces

2.1 Classifying complex surfaces

2.2 Decomposing surfaces. I

2.3 Decomposing elliptic surfaces

2.4 Surfaces of general type

Chapter 3. Four-manifolds and the calculus of links

3.1 Framed links and the Kirby calculus

3.2 Handlebody decompositions of 4-manifolds

3.3 Special handlebody-decompositions

Chapter 4. The $\mu$-invariants, homology spheres and fake 4-manifolds

4.1 The $\mu$-invariant

4.2 Fake 4-manifolds

4.3 Triangulating high-dimensional manifolds

Chapter 5. Surgery theory and its applications

5.1 Surgery theory in higher dimensions

5.2 Surgery and the smoothing and triangulations of manifolds

5.3 Triangulating 4-manifolds and some consequences of Rohlin's theorem 
Chapter 6. Surgery in dimension 4

6.1 Obstructions to four-dimensional surgery

6.2 Four-dimensional analogues of high-dimensional surgery theory

6.3 Simply-connected four-dimensional (stable) surgery

6.4 Non-simply-connected surgery in dimension 4

6.5 Surgery and fake 4-manifolds

6.6 Some five-dimensional $h$-cobordisms

6.7 Stable surgery and the Whitney trick

6.8 Flexible handles

Chapter 7. Four-manifolds as sub-and super-manifolds

7.1 Representing 2-dimensional homology classes of 4-manifolds

7.2 PL-representations

7.3 Surgery and embedding phenomena

7.4 Codimension 2 phenomena: Knotting

7.5 Codimension 2 phenomena II: The Zariski conjecture

Afterword

References

\section{CHAPTER 1. THE BASIC STRUCTURE OF 4-MANIFOLDS}

1.1 The theorems of Whitehead and Wall. Consider the following diagram of equivalence classes of simply-connected compact $n$-manifolds $\boldsymbol{M}^{n}$ :

$$
\begin{aligned}
&\left\{\begin{array}{c}
\text { Isomorphism } \\
\text { classes }
\end{array}\right\} \stackrel{\Phi_{1}}{\rightarrow}\left\{\begin{array}{c}
h \text {-cobordism } \\
\text { classes }
\end{array}\right\} \stackrel{\Phi_{2}}{\rightarrow}\left\{\begin{array}{c}
\text { Homotopy type and } \\
\text { characteristic classes }
\end{array}\right\} \stackrel{\Phi_{3}}{\rightarrow} \\
&\left\{\begin{array}{c}
\text { Integral cohomology and } \\
\text { characteristic classes }
\end{array}\right\}
\end{aligned}
$$

where the $\Phi_{i}$ are the obvious forgetful maps. Then if $n \geqslant 5$ one can show that $\Phi_{1}$ is 1-to-1 and $\Phi_{2}, \Phi_{3}$ are both finite-to-one.

What happens if $n=4$ ? We then have the following result of Whitehead [W 2] as sharpened by Milnor [M 1].

THEOREM 1.1. Suppose $M_{1}, M_{2}$ are compact simply connected smooth 4-manifolds. Let $L_{M_{1}}, L_{M_{2}}$ be the symmetric bilinear forms on $H^{*}\left(M_{1}\right), H^{*}\left(M_{2}\right)$ induced by the cup-product pairing. Then $M_{1}$ is homotopy equivalent to $M_{2}$ if and only if $\left(H^{*}\left(M_{1}\right), L_{M_{1}}\right)$ is isomorphic to $\left(H^{*}\left(M_{2}\right), L_{M_{2}}\right)$.

PROof. [We outline the proof given in [MH].] Let $M$ be a simply-connected 4-manifold and let $\boldsymbol{M}^{\prime}$ be $M-$ 44-ball $\}$. Then it is not difficult to see that $M^{\prime}$ has the homotopy type of a wedge of $r$ 2-spheres, where $r=\operatorname{rank} \mathrm{H}_{2}(M ; \mathrm{Z})$. Thus $M$ has the homotopy type $\bigvee_{r} S^{2} \cup_{g} D^{4}$ where $g: \partial D^{4} \rightarrow \bigvee S^{2}$ is some attaching map. In particular then the homotopy type of $M$ is completely determined by $[g] \in \pi_{3}\left(\bigvee_{r} S^{2}\right)$. Let $K=X_{i=1}^{i=r}\left(C P^{\infty}\right)_{i}$ and note that the inclusion $\bigvee_{r} S^{2} \hookrightarrow K$ gives us isomorphisms $\pi_{3}\left(\bigvee_{r} S^{2}\right) \simeq \pi_{4}\left(K, \bigvee S^{2}\right) \simeq$ $H_{4}\left(K, \bigvee S^{2}\right) \simeq H_{4}(K)$, where the last isomorphisms arise from the relative Hurewicz theorem and the homology exact sequence of $\left(K, \bigvee S^{2}\right)$. But calculating $H_{4}(K)$ we find that its elements consist precisely of the isomorphism classes of bilinear forms on $H_{2}\left(\bigvee_{i=1}^{r} S^{2}\right) \approx H_{2}(M)$. Thus the attaching maps $[g] \in \pi_{3}\left(\bigvee S^{2}\right)$ are in 1-to-1 correspondence with the appropriate 
isomorphism classes and so the theorem is proven.

In dimension 4 we can therefore now contract our diagram to

$$
\begin{aligned}
&\left\{\begin{array}{c}
\text { diffeomorphism } \\
\text { classes }
\end{array}\right\} \stackrel{\Phi_{1}^{\prime}}{\rightarrow}\left\{\begin{array}{c}
h \text {-cobordism } \\
\text { classes }
\end{array}\right\} \stackrel{\Phi_{2}^{\prime}}{\rightarrow}\left\{\begin{array}{c}
\text { Homotopy } \\
\text { type }
\end{array}\right\} \stackrel{\Phi_{3}^{\prime}}{\rightarrow} \\
&\left\{\begin{array}{c}
\text { Cohomology } \\
\text { ring }
\end{array}\right\}
\end{aligned}
$$

and note that $\Phi_{3}^{\prime}$ is always 1-to-1. (We note that in dimension 4 the only nontrivial Pontryagin class $p_{4}(M)$ of $M$ is uniquely determined by the signature $\sigma(M)$ of $M$ which in turn is uniquely determined by $L_{M}$. Thus we need not bother with Pontryagin class data in (2).)

What about $\Phi_{2}^{\prime}$ ? We have the following improvement of Whitehead's result by Novikov and Wall [N], [Wa 2]:

$$
\text { " } \Phi_{2}^{\prime} \text { above is 1-to-1". }
$$

More precisely

THEOREM 1.2 [N], [Wa 2]. Suppose that $M_{1}, M_{2}$ are compact simply-connected 4-manifolds. Then $M_{1}$ is $h$-cobordant to $M_{2}$ if and only if $L_{M_{1}}$ is isomorphic to $L_{M_{2}}$.

Proof. We shall divide the proof up into a few steps. As a preliminary step we review the definition of the bordism groups $\Omega_{*}^{\text {SO }}, \Omega_{*}^{\text {SPIN }}$. Recall that $\Omega_{*}^{\text {SO }}$ $\left(\Omega_{*}^{\text {SPIN }}\right)$ is the group of oriented (spin) cobordism classes of oriented (spin) manifolds. That is, elements of $\Omega_{*}^{\text {SO }}\left(\Omega_{*}^{\text {SPIN }}\right)$ are equivalence classes of oriented (spin) manifolds under the equivalence $M_{1}^{n} \sim M_{2}^{n}$ if there exists an oriented (spin) cobordism between them (i.e., the manifold $W^{n+1}$ with $\partial W=M_{1} \cup$ $M_{2}$ is an oriented (spin) manifold and the boundaries have the induced orientations. Thus $\partial W=M_{1}-M_{2}$ as oriented manifolds). The group operation in $\Omega_{*}^{\text {SO }}\left(\Omega_{*}^{\text {SPIN }}\right)$ is disjoint union and the identity element can be taken to be a point. Inverses are obtained by reversing orientation. $\Omega_{*}^{\text {SO }}\left(\Omega_{*}^{\text {SPIN }}\right)$ is a graded group, $\Omega_{*}^{S}=\oplus \Omega_{i}^{S}, \Omega_{i}^{S}$ is the group of cobordism classes of $i$-dimensional $S$-manifolds $\left(S=S O\right.$ or SPIN). (Note that the identity element in $\Omega_{i}$ is just $S^{i}$ and since $S^{i}$ bounds $D^{i+1}$ saying that $M^{i}$ is cobordant to zero is equivalent to saying that $M^{i}$ is the oriented boundary of a manifold $W^{i+1}$.)

By a direct calculation using the methods in [Thm] one can show $\Omega_{4}^{\text {SO }} \stackrel{0}{\approx} \mathbf{Z}$; $\Omega_{4}^{\text {SPIN }} \approx \mathbf{Z}$ and the "forgetful map" $\Omega_{4}^{\text {SPIN }} \rightarrow \Omega_{4}^{\text {SO }}$ is essentially $\mathbf{Z} \stackrel{16}{\rightarrow} \mathbf{Z}$. (The isomorphism $\sigma$ above is simply $M \rightarrow \sigma(M) \in \mathbf{Z}$.)

Now clearly if $M_{1}$ is $h$-cobordant to $M_{2}$ we have $L_{M_{1}}$ is isomorphic to $L_{M_{2}}$. Therefore to prove the converse let us suppose $\alpha: H_{2}\left(M_{1}\right) \rightarrow H_{2}\left(M_{2}\right)$ is an inner-product isomorphism. Let $N=M_{1} \#\left(-M_{2}\right)$. Then $H_{2}(N ; \mathbf{Z}) \approx$ $H_{2}\left(M_{1} ; \mathbf{Z}\right) \oplus H_{2}\left(M_{2} ; \mathbf{Z}\right)$ and $L_{N_{1}} \simeq L_{M_{1}} \oplus\left(-L_{M_{2}}\right)$. Thus $\sigma(N)=\sigma\left(M_{1}\right)-$ $\sigma\left(M_{2}\right)=0$. Therefore $N$ is cobordant to $S^{4}$ and thus bounds an oriented 5-manifold $Z$. Furthermore by our remarks on the map $\Omega_{4}($ Spin $) \rightarrow \Omega_{4}$ we see that if $N$ is spin we may assume that about $Z$ also.

Step (1). We may without loss of generality suppose that $Z$ has the homotopy type of a wedge of 2-spheres.

Proof. Recall that if $M^{n}$ is an $n$-manifold and $f: S^{p} \times D^{n-p}$ is an 
embedding with $\alpha$ being the homotopy class represented by $f\left(S^{p} \times 0\right)$ then the spherical modification of $M^{n}$ along $\alpha$ is the manifold

$$
\chi(M)=\overline{M-f\left(S^{p} \times D^{n-p}\right)} \cup_{S^{p} \times S^{n-p-1}} D^{p+1} \times S^{n-p-1} .
$$

It is easy to see that $\alpha$ goes to zero in $\chi(M)$ and in [M3] it is shown (see also Chapter 5) that if $\operatorname{dim} V \geqslant 4$ any manifold $V$ can be modified by a sequence of spherical modifications to obtain a 1 -connected manifold $V^{\prime}$. Note that such modifications do not affect $\partial V$. (Spherical modifications are also called surgeries.) Thus we can always so modify $Z$ until it is 1 -connected. We continue to call the modified manifold $Z$. Clearly what must first be shown is that further spherical modifications can be performed on $Z$ until $H_{2}(Z, N)$ vanishes since by duality this would mean $H_{3}(Z)=0$.

Consider the homology exact sequence

$$
0 \rightarrow H_{3}(Z) \rightarrow H_{3}(Z, N) \rightarrow H_{2}(N) \rightarrow H_{2}(Z) \stackrel{j_{*}}{\rightarrow} H_{2}(Z, N) \rightarrow 0 .
$$

It is not difficult to show using $w_{2}(N)=0 \Rightarrow w_{2}(Z)=0$ that if $y_{1}, \ldots, y_{t}$ generate the infinite part of $H_{2}(Z, N)$ then there exist elements $x_{1}, \ldots, x_{t} \in$ $H_{2}(Z)$ representable by embeddings $f_{i}: S^{2} \times D^{3} \rightarrow \operatorname{int}(Z)$ and such that $j_{*}\left(x_{i}\right)=y_{i}$. Surgering $Z$ along $x_{i}$ has the effect of 'killing' all the $x_{i}$ and thus all the $y_{i}$. We can thus assume without loss of generality that $Z$ has been so modified. Thus $H_{2}(Z, N)$ is a finite group. Note that since $N$ is simply connected, $H_{2}(N)$ has no torsion and by Poincare duality and the universal coefficient theorem we may assume $H_{2}(Z)$ is infinite.

One can now show that, provided $H_{2}(Z, N) \neq \varnothing$, there exist elements $u_{i} \in H_{2}(Z)$ representable by embeddings $g_{i}: S^{2} \times D^{3} \rightarrow \operatorname{int}(Z)$ with $u_{i}$ generating an infinite direct summand of $H_{2}(Z)$ and $j_{*}\left(u_{i}\right) \neq 0$. Again via surgery we can 'kill' $u_{i}$ and thus $j_{*}\left(u_{i}\right)$, thereby reducing the order of $H_{2}(Z, N)$. By induction one can thus reduce $H_{2}(Z, N)$ to the zero group. (There is actually a small technical difficulty here if $\mathrm{rk} H_{2}\left(M_{1}\right)=1$ which we suppress. See [Wa 2, pp. 143, 146] for details.) Again call the modified manifold $Z$ and note that $H_{2}(Z)$ now must be a free abelian group and so $Z$ has the desired homotopy type.

Step (2). There exists a manifold $H$, diffeomorphic to a boundary-connected sum of $D^{3}$-bundles over $S^{2}$, embedded as a deformation retract in $Z$. Furthermore, the closure of $Z-H$ gives an $h$-cobordism of $N$ to $\partial H$.

Proof. To construct $H$, first embed a disc $D^{5}$ in $Z$ and then embed discs $D_{i}^{2}$ in $Z$ with interiors avoiding $D^{5}$ and boundaries lying on it such that their homology classes represent generators of $H_{2}(Z)$. (Such embedding are always possible via a general position argument which also guarantees that we may take the $D_{i}^{2}$ disjoint.) Let $H$ be a neighborhood of $D^{5} \cup \cup{ }_{i} D_{i}^{2}$. Then it is easy to see that $H$ is as claimed above. A straightforward calculation of homology groups shows that $Z-H$ is an $h$-cobordism.

Step (3). There exists a unique nontrivial orientable $S^{2}$-bundle over $S^{2}$ (which we denote by $S^{2} \times S^{2}$ ) and $\partial H$ is diffeomorphic to a connected sum

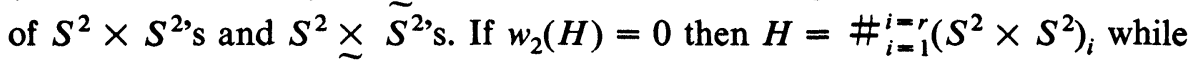
if $w_{2}(H) \neq 0$ then $H=\mathbb{Z}_{i=1}^{i=r}\left(S^{2} \underset{\sim}{\times} S^{2}\right)_{i}$.

Proof. We note that orientable $S^{2}$ - and $D^{3}$-bundles over $S^{2}$ are classified 
by $\pi_{1}(\mathrm{SO}(3)) \approx \mathbf{Z}_{2}$ and thus there is only one nontrivial bundle of that type, which we denote by $S^{2} \underset{\sim}{S^{2}}$, resp. $D^{3} \underset{\sim}{\times} S^{2}$. See [Stn, \$26]. (If $P$ denotes the complex projective plane $\mathbf{C} P^{2}$ with its standard orientation and $Q$ denotes $\mathbf{C} P^{2}$ with orientation opposite to the usual, then a straightforward construc-

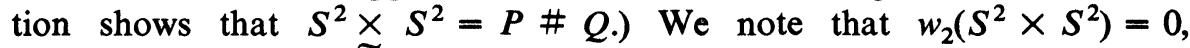
$w_{2}\left(S^{2} \times S^{2}\right) \neq 0$. Thus if $A$ is any connected sum of orientable $S^{2}$-bundles over $S^{\widetilde{2}}$, then $w_{2}(A)=0$ if and only if $A=\#_{i=1}^{i=r} S^{2} \times S^{2}$. We also note that if $M$ is an arbitrary 4-manifold with $w_{2}(M) \neq 0$ then $M \#\left(S^{2} \times S^{2}\right)$ is diffeomorphic to $M$ \# $\left(S^{2} \times S^{2}\right)$. See [Wa 1]. Thus any connected sum $A$ of $S^{2} \times S^{2}$ and $S^{2} \times S^{2}$ with $w_{2}(A) \neq 0$ is diffeomorphic to a connected sum $\# S^{2} \times S^{2}$. Clearly the boundary of a boundary-connected sum of $D^{3}$-bundles over $S^{2}$ is a connected sum of $S^{2}$-bundles over $S^{2}$ and thus $\partial H$ is as desired.

Step (4). $M_{1}$ is $h$-cobordant to $M_{2}$.

Proof. (See Figure 1.1.) Let $W$ be the closure of $Z-H$. Thus $W$ is an $h$-cobordism between $N$ and $\partial H$. Now $N=M_{1}-\dot{D}^{4} \cup\left(S^{3} \times I\right) \cup\left(M_{2}-\right.$ $\left.\dot{D}_{4}\right)$. We can thus attach a $D^{4} \times I$ to $W$ 'filling in' the tube $S^{3} \times I$ of the connected sum above and obtain an orientable 5-manifold $W^{\prime}$ with $\partial W^{\prime}$ having $M_{1}, M_{2}$ and $\partial H$ as disjoint boundary components. Thus to construct an $h$-cobordism we will need to attach $H$ to $W^{\prime}$ along $\partial H$ using an autodiffeomorphism $f$ such that if $R_{f}=W^{\prime} \cup_{f} H$ then $H_{*}\left(R_{f}, M_{1}\right)=0$. (This condition on homology groups guarantees of course that $M_{1} \hookrightarrow R_{f}$ is a homotopy equivalence.) Let $K$ be the subgroup of $H_{2}(\partial H) \approx H_{2}(N) \approx H_{2}\left(M_{1}\right) \oplus$ $H_{2}\left(M_{2}\right)$ equal to $\left\{(X, \alpha X) \mid X \in H_{2}\left(M_{1}\right)\right\}$ (recall $\alpha: H_{2}\left(M_{1}\right) \rightarrow H_{2}\left(M_{2}\right)$ is our original isometry). Let $L$ be the kernel of $H_{2}(\partial H) \stackrel{i_{*}}{\rightarrow} H_{2}(H)$, where $i$ is the inclusion map. Notice that both $K$ and $L$ satisfy rank $K=\operatorname{rank} L=\frac{1}{2}$ rank $H_{2}\left(M_{1}\right)$ and $Z \cdot Z=0$ for any $Z \in K$ or $Z \in L$ (where $\cdot$ is the intersection pairing on $\left.\mathrm{H}_{2}(\partial H) \approx \mathrm{H}_{2}(\mathrm{~N})\right)$. A straightforward calculation on homology shows that if $K=L$ then $H_{*}\left(R, M_{1}\right)=0$ and we have the $h$-cobordism we sought. Thus the proof reduces to showing that there is an autodiffeomorphism $f: \partial H \rightarrow \partial H$ with $f_{*}(K)=L$. This is demonstrated in Chapter 6, Theorem 6.26 .

Thus Theorem 2 shows us that for simply-connected 4-manifolds their integral cohomology alone determines their $h$-cobordism type. This is definitely false in higher-dimensions. See [Wa 3].

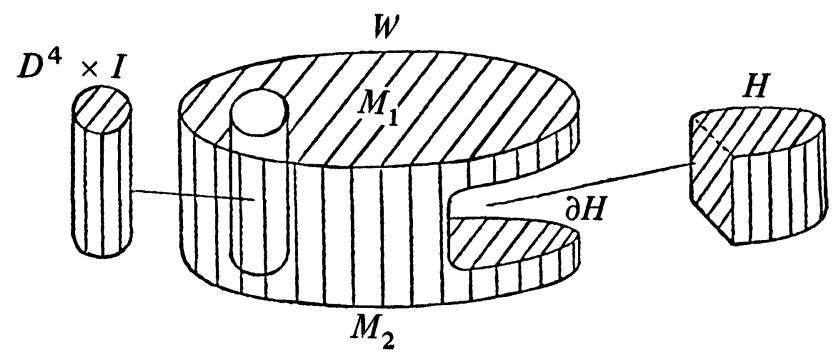

FIgURE 1.1 
Step (3) in the above proof is important enough to extract as a separate lemma. The proof is straightforward except for (iv)c which we prove in Chapter 6.

Lemma 1.3. (i) There exist two distinct orientable $S^{2}$-bundles over $S^{2}$. The trivial one $S^{2} \times S^{2}$ and the nontrivial one $S^{2} \underset{\sim}{\times} S^{2}$.

(ii) $S^{2} \times S^{2}$ is diffeomorphic to $P \# Q$, where $P=\mathbf{C} P^{2}$ with its usual orientation and $Q$ is $\mathbf{C} P^{2}$ with orientation opposite to the usual.

(iii) $w_{2}\left(S^{2} \times S^{2}\right)=0, w_{2}\left(S^{2} \underset{\sim}{\times} S^{2}\right) \neq 0$.

(iv) If $M$ is any compact connected 4-manifold then

(a) $w_{2}(M)=0 \Rightarrow w_{2}\left(M \# S^{2} \times S^{2}\right)=0$,

(b) $w_{2}\left(M \# S^{2} \underset{\sim}{S^{2}}\right) \neq 0$,

(c) $w_{2}(M) \neq 0 \Rightarrow M \# S^{2} \times S^{2}=M \# S^{2} \underset{\sim}{\times} S^{2}$.

Lastly let us consider

$\Phi_{1}^{\prime}:\{$ diffeomorphism classes $\} \rightarrow\{h$-cobordism classes $\}$

for simply-connected 4-manifolds. (We recall that by a theorem of Cairns [Ca 2], [Ca 3], [Hi 1] every PL 4-manifold has a unique DIFF structure and so in dimension 4, PL $=$ DIFF and we shall use the two interchangeably.) Now in dimension $n>4$ the map $\Phi_{1}^{\prime}$ above is 1-to-1 as a consequence of the $h$-cobordism theorem (see [Sm], [M 4] in the smooth case and [Z 1], [St 1] in the PL case. We shall continue our discussion in the PL case but our theorems and proofs could be redone in the other categories also. We follow the treatment in $[\mathbf{R S}]$.)

We have

THEOREM H ( $h$-COBORDISM THEOREM). Let $\left(W^{n+1}, M_{0}^{n}, M_{1}^{n}\right)$ be an $h$-cobordism with $\pi_{1}\left(W^{n+1}\right)=0$. Then if $n \geqslant 5, W^{n+1}$ is isomorphic to $M_{0}^{n} \times I$.

We will run through a PL-version of the proof of Theorem $\mathrm{H}$. Thus we will use handles and handlebody-decompositions. (A proof of the smooth version using Morse functions can be found in [M 4].)

We recall that if $W^{m}$ is an $m$-manifold (all our manifolds and maps are PL) and $H$ an $m$-ball with $W \cap H \subset \partial W$ then $H$ is called a $p$-handle on $W$ if there exists an isomorphism $h: D^{p} \times D^{q} \rightarrow H$ with $h\left(\partial D^{p} \times D^{q}\right)=H \cap W$. Clearly then setting $f=h \mid \partial D^{p} \times D^{q}$ we see that $W^{\prime}=W \cup H \approx W \cup \cup_{f} D^{m}$ is also an $m$-manifold. We say $W^{\prime}$ is formed from $W$ by attaching a $p$-handle and sometimes write $W^{\prime}=W \cup_{f} H^{(p)}$. We refer to $h\left(D^{p} \times 0\right)$ as the core and $h\left(0 \times D^{q}\right)$ as the cocore of $H . h\left(\partial D^{p} \times 0\right)$ is the attaching sphere ( $a$-sphere) and $h\left(0 \times D^{q}\right)$ the belt sphere ( $b$-sphere). $h$ is called the characteristic map and $f$ the attaching map. Now if $\left(W, M_{0}, M_{1}\right)$ is a cobordism and $H$ is a handle on $W$ then $H$ is a handle on the cobordism if $H \cap W \subset M_{1}$. Letting $M_{2}=\partial W^{\prime}-M_{0}$ where $W^{\prime}=W \cup H$ we obtain a new cobordism $\left(W^{\prime}, M_{0}, M_{2}\right)$ (see Figure 1.2) which we say is obtained from the original cobordism by attaching a handle. We will sometimes write a cobordism $W^{\prime}=W \cup H^{\left(r_{1}\right)} \cup H^{\left(r_{2}\right)} \cup \cdots \cup H^{\left(r_{m}\right)}$ to mean $W^{\prime}$ is a cobordism obtained by successively adding the handle $H^{\left(r_{j}\right)}$ to the cobordism $W \cup H^{\left(r_{1}\right)}$ $\cup \cdots \cup H^{\left(r_{j-1}\right)}$. (Note that if $W^{\prime}=W \cup H^{r}$ as above, then $M_{2}$ can easily 
be seen to be diffeomorphic to

$$
\chi\left(M_{1}\right)=M_{1}-f\left(S^{r-1} \times D^{n-r+1}\right) \cup_{S^{r-1} \times S^{n-r}} D^{r} \times S^{n-r},
$$

where $f$ is the attaching map of $H^{r}$.) (See Figure 1.2.) Thus attaching a handle to $W$ along $M_{1}$ has the effect of surgering $M_{1}$ along $f\left(S^{r-1} \times D^{n-r+1}\right)$. If $W^{\prime n+1}=W \cup H^{(r)} \cup H^{(r+1)}$ then if $S_{1}^{n-r}=b$-sphere of $H^{(r)}=h^{(r)}(0 \times$ $\left.\partial D^{n-r+1}\right)$ and $S_{2}^{r}=a$-sphere of $H^{(r+1)}=h^{(r+1)}\left(\partial D^{r+1} \times 0\right)$ then $S_{1}$ and $S_{2}$ lie in $M_{2}=\partial\left(W \cup H^{(r)}\right)-M_{0}$ and have complementary dimensions. Thus we can define $\varepsilon\left(H^{(r)}, H^{(r+1)}\right)$, the incidence number of $H^{(r)}$ and $H^{(r+1)}$ as the algebraic intersection number $S_{1} \cdot S_{2}$. (See [RS, p. 77] for details.) Now if $S_{1}$ and $S_{2}$ intersect transversely in only one point we say that $H^{(r)}$ and $H^{(r+1)}$ are complementary handles. If $S_{1}$ and $S_{2}$ have algebraic intersection \pm 1 (so that $S_{1}$ and $S_{2}$ by general position intersect transversely in $2 m+1$ points (for some $m \geqslant 0)$ with $m$ pairs of $(+1,-1)$ intersections), we shall say $H^{(r)}$ and $H^{(r+1)}$ are algebraically complementary.

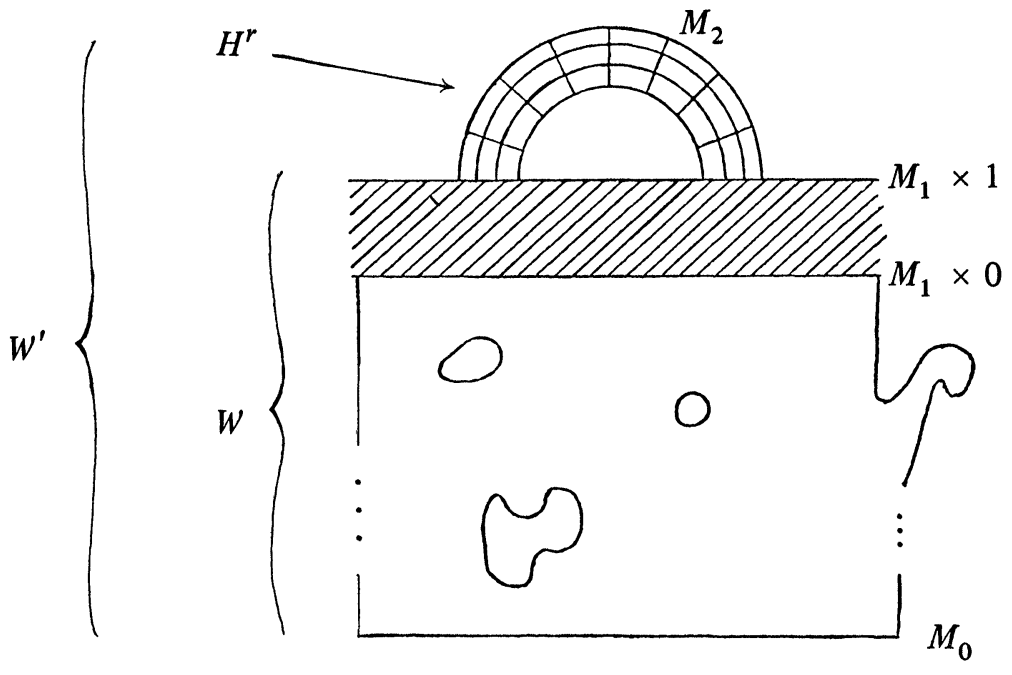

Note that $M_{2}=\chi\left(M_{1}\right)=M_{1}-f\left(S^{r-1} \times D^{n-r+1}\right) \cup_{S^{r-1} \times S^{n-r}} D^{r} \times S^{n-r}$.

FIGURE 1.2

If $W^{n+1}$ is a closed manifold, then a handle-decomposition of $W$ is a decomposition $W=H_{0} \cup H_{1} \cup \cdots \cup H_{t}$ where $H_{0}$ is an $(n+1)$-ball and $H_{i+1}$ is a handle on $W_{i-1}=\cup_{j<i} H_{j}$. If $\left(W, M_{0}, M_{1}\right)$ is a cobordism then a handle-decomposition of $W$ on $M_{0}$ is a decomposition $W=M_{0} \times I \cup H_{1}$ $\cup \cdots \cup H_{t}$ where $M_{0} \times I$ is regarded as a cobordism in the obvious fashion and $H_{j+1}$ is a handle on the cobordism $M_{0} \times I \cup \cup{ }_{i<j} H_{i}$. Using the PL-structure on $W$ it is possible to show that every PL manifold or cobordism admits a handle-decomposition. (This is not true for TOP manifolds, see Chapter 5 and [Sb 1].)

There are a few basic geometric principles involved in manipulating handle-decompositions.

PRINCIPLe 1 (ReORDeRING). If $W^{\prime}=W \cup H^{(r)} \cup H^{(s)}$ with $s \leqslant r$ then $W^{\prime} \simeq W \cup H^{(s)} \cup H^{(r)}$ with $H^{(s)}$ disjoint from $H^{(r)}$. 
PRINCIPLe 2 (HANDle ADdition). Let $W^{n+1}=W \cup H^{(r-1)} \cup H_{1}^{r} \cup H_{2}^{r}$ with $r \geqslant 2$ and $n \geqslant r+1$. Then $\partial\left(W \cup H^{(r-1)}\right)-M_{0}$ is simply-connected implies that there exists a new handle-decomposition $\tilde{W}^{n+1}=W \cup H^{(r-1)}$ $\cup H_{1}^{r} \cup H_{3}^{r}$ of $W^{n+1}$ with

$$
\varepsilon\left(H_{3}^{r}, H^{(r-1)}\right)=\varepsilon\left(H_{2}^{r}, H^{(r-1)}\right) \pm \varepsilon\left(H_{1}^{r}, H^{(r-1)}\right)
$$

( $H_{3}^{r}$ is called the handle obtained by adding $H_{1}^{r}$ to (or subtracting $H_{1}^{r}$ from) $\left.H_{2}^{r}\right)$.

Principle 3 (Geometric Cancellation and Introduction). If $W^{\prime}=W$ $\cup H^{(r)} \cup H^{(r+1)}$ and $H^{(r)}$ and $H^{(r+1)}$ are complementary handles then there is an isomorphism $h: W^{\prime} \rightarrow W$ which is the identity outside a neighborhood of $H^{(r)} \cup H^{(r+1)}$. Conversely if $W^{\prime n+1}=W \cup B^{n+1}$, where $B^{n+1}$ is an $n+1$ ball with $B^{n+1} \cap W=B^{n+1} \cap M_{1}=\mathrm{a}$ face $B_{1}$ of $B$, then we can write $W^{\prime}=W \cup H^{(r)} \cup H^{(r+1)}$ with $H^{(r)}$ and $H^{(r+1)}$ complementary.

Principle 4 (Algebraic Cancellation) [RS, p. 79]. Suppose $W^{\prime}=W^{n+1}$ $\cup H^{(r)} \cup H^{(r+1)}$ and $M_{1}$ is simply-connected, $n-r \geqslant 3, r \geqslant 2$ and $n \geqslant 5$. Then if $H^{(r)}$ and $H^{(r+1)}$ are algebraically complementary then $W^{\prime} \simeq W$.

There is a fundamental difference between Principles 1,2,3 and Principle 4. The first three principles are proven using rather elementary techniques of PL-geometry such as general position and regular neighborhoods [see RS pp. 76-80]. They are true without any dimensional restrictions. Principle 4, however, is somewhat deeper and is not universally true. Its proof is a consequence of the Whitney trick which states

Whitney Lemma (Simply-connected) [RS, p. 68], [Whit 1]. Suppose $P^{p}$, $Q^{q}$, are oriented submanifolds of the oriented manifold $M^{m}$ and that $p+q=$ m. Suppose further that $P^{p} \pitchfork Q^{q}$ and $p, q \in P^{p} \cap Q^{q}$ with $(P \cdot Q)_{p}=-(P$. $Q)_{q}$, where $(P \cdot Q)_{x}$ is the algebraic intersection index of $P$ and $Q$ at $x$. Then if $p>3, q \geqslant 3$ and $\pi_{1}(M)=0$ or $p=2, q \geqslant 3$ and $\pi_{1}(M-Q)=0$ there exists an isotopy of $M$ carrying $P$ to $P^{\prime}$ with $P^{\prime} \pitchfork Q$ and $P^{\prime} \cap Q=P \cap Q-\{p\}$ $-\{q\}$. Moreover the isotopy has support in a compact set not meeting any other intersection points.

Corollary WL1. Under the above hypothesis $P$ can be isotoped to $P^{\prime}$ with $P^{\prime} \pitchfork Q$ and if $n=P \cdot Q$ then $P^{\prime} \cap Q=\left\{x_{1}, \ldots, x_{|n|}\right\}$ with $\left(P^{\prime} \cdot Q\right)_{x_{i}}=$ sign $n$ if $n \neq 0$ and $P^{\prime} \cap Q=\varnothing$ if $n=0$.

Thus the Whitney trick tells us that with suitable dimensional restrictions we can always replace algebraic intersection by geometric intersection.

We can also use the Whitney trick to eliminate self intersections of homology classes. More precisely we have

COROLLARY WL2 [M 3]. Suppose $W$ is a simply-connected manifold and $V$ is a compact, connected manifold. Then (i) If $2 \operatorname{dim} V<\operatorname{dim} W$, any map $f$ : $V \rightarrow W$ is homotopic to an immersion $f^{\prime}: V \rightarrow W$ having only a finite number of transversal double points as singularities.

(ii) If $\operatorname{dim} W \geqslant 5$ then $f^{\prime}$ is in turn homotopic to an embedding.

The Whitney trick does not work however if $p=q=2$. This is the cause for the anomalous behavior of dimension 4 in general, and the breakdown of 
the proof of the $h$-cobordism theorem in particular. We shall discuss further implications of the failure of the Whitney trick in \$6.7. At this point we shall go through the proof of the $h$-cobordism theorem and see where the Whitney lemma is used.

Thus suppose $\left(W^{n+1}, M_{0}^{n}, M_{1}^{n}\right)(n \geqslant 4)$ is a simply-connected $h$-cobordism. Then $W^{n+1}$ has a handle-decomposition which, using Principle 1 and the triviality of the product cobordism, can be written as

$$
\begin{aligned}
W^{n+1}= & M_{0} \times I \cup\left(\bigcup^{a_{0}} H_{i}^{0}\right) \cup\left(\bigcup^{a_{1}} H_{j}^{1}\right) \\
& \cup \cdots \cup\left(\bigcup^{a_{n}} H_{k}^{n}\right) \cup\left(\bigcup^{a_{n+1}} H_{p}^{n+1}\right)
\end{aligned}
$$

where $H_{k}^{m}$ is an $m$-handle and all the handles we added are disjoint. Then using the connectedness of $W^{n+1}$ we can show that every 0 -handle can be cancelled by an appropriate 1-handle. Thus we can cancel the $a_{0} 0$-handles by $a_{0}$ 1-handles (for homological reasons we have $a_{1} \geqslant a_{0}$ ) to obtain a new handle-decomposition with no 0 -handles and $a_{1}-a_{0} 1$-handles.

Now notice that if $W=M_{0} \times I \cup H_{1} \cup \cdots \cup H_{t}$ is a handle-decomposition of $W$ then we can add on the trivial cobordism $M_{1} \times I$ to $W$ without altering it. Thus $W=M_{0} \times I \cup H_{1} \cup \cdots \cup H_{t} \cup M_{1} \times I$. Furthermore, using the interchange automorphism $t: I^{p} \times I^{q} \rightarrow I^{q} \times I^{p}$ we define $W_{i+1}^{*}=$ $M_{1} \times I \cup \cup\left\{H_{j} \mid j \geqslant i+1\right\}$ and see that we can regard the $p$-handle $H_{j}$ as a $q$-handle $H_{i}^{*}$ on $W_{i+1}^{*}$ with charactereristic map $h_{i}^{*}=h_{i} \circ t$. Thus $W_{1}^{*}$ gives a dual handle-decomposition of our cobordism wherein every $p$-handle becomes an $(n+1-p)$-handle. In particular then cancelling 0 -handles by 1-handles in the dual handle-decomposition translates into cancellation of $(n+1)$ - by $n$-handles in the original decomposition. Thus by using the connectivity of $W$ we can always assume (provided $M_{0} \neq \varnothing, M_{1} \neq \varnothing$ ) that our cobordism has no 0 - or $(n+1)$-handles. Using the simple-connectivity and Principle 2 (but not necessarily the Whitney trick) we can also always exchange 1-handles for 2-handles and $n$-handles for $(n-1)$-handles and thus we can always assume that $a_{1}=a_{n}=0$ in (3) above. To proceed further we must now resort to a use of Principle 4. Using the fact that $W$ is simply-connected and $M_{0} \hookrightarrow W$ is a homotopy equivalence we can show that, for every $s$, $2<s<n-3$, if $H^{s}$ is an $s$-handle then by handle additions and subtractions (Principle 2$)$ of $(s+1)$-handles we can produce an $(s+1)$-handle $H^{(s+1)}$ which is algebraically complementary to $H^{(s)}$. Now if $n \geqslant 5$ we can resort to Principle 4 which will then allow us to cancel $H^{s}$ and $H^{s+1}$. Thus we can get rid of all the $s$-handles $2<s<n-3$ until we are left only with $(n-2)$-handles and $(n-1)$-handles. By the fact that $M_{0} \hookrightarrow W$ is a homotopy equivalence there must be exactly as many $(n-2)$-handles as $(n-1)$ handles left and dualizing the above procedure we can add and subtract the $(n-2)$-handles among themselves until they are all algebraically complementary to $(n-1)$-handles. Applying Principle 4 then eliminates all the remaining $(n-2)$ and $(n-1)$-handles and we are thus left with $W^{n+1} \approx M_{0} \times I$ as desired, provided $n \geqslant 5$. 
What happens if $n=4$ ? Then we can still eliminate 0-, 1-, 4- and 5-handles to get

$$
W^{5}=M_{0} \times I \cup \bigcup_{i=1}^{k_{1}} H_{i}^{2} \cup \bigcup_{j=1}^{k_{2}} H_{j}^{3}
$$

and since $M_{0} \hookrightarrow W_{5}$ is a homotopy equivalence we must have $k_{1}=k_{2}$. Note that we have, even in principal, no control of how large $k_{1}$ must be in order that $\left(3^{\prime}\right)$ be valid.

Actually by being more careful in our proof we could have proven the following $s$-cobordism theorem.

THEOREM S ( $s$-COBORDISM THEOREM). Let $\left(W^{n+1}, M_{0}, M_{1}\right)$ be an $s$-cobordism. Then if $n \geq 5, W^{n+1}$ is isomorphic to $M_{0}^{n} \times I$.

The basic modification in our proof consists of:

(1) using a non-simply-connected version of the Whitney lemma to obtain a non-simply-connected version of the algebraic cancellation principle, and

(2) taking care of base points in all our definitions and operations.

We will not go through the proof here but refer the interested reader to [RS, p. 88]. We will, however, state for future use the non-simply-connected version of Whitney's lemma on which the proof is based. See [RS, p. 72].

We first must redefine the notion of an algebraic intersection. Recall that if $M^{m}$ is an oriented manifold and $P^{p}, Q^{q}(p+q=m)$ are oriented submanifolds then $P \cdot Q$ is essentially the homological intersection of the homology classes $[P],[Q]$ in $H_{m}(M ; \mathbf{Z}) \approx \mathbf{Z}$.

More geometrically, we can by general position always suppose that $P$ and $Q$ intersect transversely in a finite number of points $p_{1}, \ldots, p_{n}$. Then we define the intersection number $\varepsilon\left(p_{i}\right)$ of $P$ and $Q$ at $p_{i}$ as being +1 if the local orientation of $P$ and $Q$ at $p_{i}$ give the orientation of $M$ at $p_{i}$ and -1 otherwise. Then $P \cdot Q=\sum_{i=1}^{i=n} \varepsilon\left(p_{i}\right)$ and $(P \cdot Q)_{p_{i}}=\varepsilon\left(p_{i}\right)$. Now suppose $\pi_{1}(M) \neq 0$ and $P, Q$ are simply-connected and oriented. Let * be a base point for $M$ and $e_{P}$, $e_{Q}$ fixed paths in $M$ to the base points of $P, Q$. Fix a local orientation $\mathscr{F}$ for $M$ at $*$. We then define $\varepsilon(x)$ for $x \in P \cap Q$ as the elements $\pm g$, where $g \in \pi_{1}(M)$ is the element determined by $e_{P} \rho \tau e_{Q}^{-1}, \rho$ a path in $P$ from its base point to $x$ and $\tau$ a path in $Q$ from $x$ to the base point of $Q$. The \pm depends on comparing the local orientation $\mathscr{F}_{P}$ of $M$ at $p$ obtained by transporting * to $x$ via $e_{P} \rho$ with the local orientation given by $P$ and $Q$ at $x$. Then we define $\varepsilon(P, Q) \in \mathbf{Z}\left[\pi_{1}(M)\right]$ to be $\Sigma_{x \in P \cap Q} \varepsilon(x)$.

Then the non-simply-connected version of Whitney's lemma is

WhitNey LeMMa (NON-SIMPLY-CONNECTED VERSION) [RS, p. 72]. Suppose $P^{p}, Q^{q}$ are oriented connected locally flat submanifolds of the connected manifold $M^{m}$ with $m=p+q$. Suppose $P^{p} \pitchfork Q^{q}$ and $\varepsilon(x)$ for $x \in P \cap Q$ is defined as above. Let $x, y \in P^{p} \cap Q^{q}$ with $\varepsilon(x)=-\varepsilon(y)$. Then if either $p>3$, $q \geqslant 3$ or $p=2, q \geqslant 3$ and $\pi_{1}(M)=\pi_{1}(M-Q)$ there exists an isotopy of $M$ carrying $P$ to $P^{\prime}$ with $P^{\prime} \pitchfork Q=P \cap Q-\{x\}-\{y\}$. The isotopy has support in a compact set not meeting any other intersection points.

One also has the obvious analogues of Corollaries WL1 and WL2. 
One then must replace Principle 4 by

PRINCIPLE 4'. Suppose $W^{\prime}=W^{n+1} \cup H^{(r)} \cup H^{(r+1)}$ and $\pi_{1}\left(M_{1}\right) \simeq \pi_{1}\left(M_{2}\right)$ $\simeq \pi_{1}(W)$ where $M_{2}=\partial\left(W \cup H^{(r)}\right)-M_{0}$ and $M_{1}=\partial W-M_{0}$. If $2 \leqslant r \leqslant$ $n-3$ and $\varepsilon\left(H^{(r+1)}, H^{(r)}\right)= \pm g$, where $g \in \pi$, then $W^{\prime} \approx W$.

Let us now return to the case of 4-dimensional manifolds. In particular what information can be extracted from ( $\left(3^{\prime}\right)$ above? We have the following theorem of Wall [Wa 2].

THEOREM 1.4. Suppose $M_{0}, M_{1}$ are $h$-cobordant simply-connected compact 4-manifolds. Then there exists an integer $k \geqslant 0$ such that $M_{1} \# k\left(S^{2} \times S^{2}\right)$ is diffeomorphic to $M_{2} \# k\left(S^{2} \times S^{2}\right)$.

Proof. We first require a lemma.

Lemma 1.5. Suppose $X$ is a 4-manifold and $c$ is a null homotopic circle in $X$. Let $f: S^{1} \times D^{3} \rightarrow X$ be an embedding with $f\left(S^{1} \times 0\right)=c$. Let $Y$ be $X$ surgered along $c$ via $f$. Then $Y=X \# S^{2} \times S^{2}$ or $X \# S^{2} \times S^{2}$. We can always choose $f$ so that $Y=X \# S^{2} \times S^{2}$.

Proof. Since $X$ is a 4-manifold we may by [RS] assume that $c$ lies in a 4-disc $D^{4} \subset X$. Then

$$
\begin{aligned}
Y & =\overline{X-D^{4}} \cup D^{4}-f\left(S^{1} \times D^{3}\right) \cup D^{2} \times S^{2} \\
& =\overline{X-D^{4}} \cup \overline{\left(D^{2} \times S^{2} \cup S^{1} \times S^{2} D^{2} \times S^{2}\right)-D^{4}} .
\end{aligned}
$$

But $D^{2} \times S^{2} \cup S^{1} \times S^{2} D^{2} \times S^{2}$ is simply an orientable $S^{2}$-bundle over $S^{2}$ and thus equals either $S^{2} \times S^{2}$ or $S^{2} \times S^{2}$. Thus $Y$ is as desired. Clearly we can always modify $f$ by adding an additional twist in $D^{3}$ as we go along $S^{1}$ so that the bundle obtained is trivial.

Suppose now that $\left(W^{5}, M_{0}, M_{1}\right)$ is the requisite $h$-cobordism. As a consequence of our discussion above $W^{5}$ admits a handlebody-decomposition

$$
W^{5}=M_{0} \times I \cup \bigcup_{i=1}^{k} H_{i}^{2} \cup \bigcup_{i=1}^{k} H_{i}^{3} \cup M_{1} \times I .
$$

Let

$$
W_{1}=M_{0} \times I \cup \bigcup_{i=1}^{k} H_{i}^{2}, \quad W_{2}=\bigcup_{i=1}^{k} H_{i}^{3} \cup M_{1} \times I .
$$

Dualizing $W_{2}$ we obtain a cobordism $W_{2}^{*}=M_{1} \times I \cup \cup_{i=1}^{k}\left(H_{i}^{3}\right)$ where each $\left(H_{i}^{3}\right)$ is a 2 -handle attached to $M_{1} \times I$ along $M_{1} \times 1$. Clearly $\partial W_{1}-M_{0}$ $=\partial W_{2}-M_{1}$. Set $M=\partial W_{1}-M_{0}$.

Now since $W$ is an $h$-cobordism we must have that $\pi_{1}(M)=\pi_{1}\left(M_{0}\right)=$ $\pi_{1}\left(M_{1}\right)$. Thus the attaching map $f: S^{1} \times D^{3} \rightarrow M_{i}$ of any 2-handle in the cobordisms above is null-homotopic. But as we mentioned before adding a 2-handle to a cobordism $\left(V^{5}, N_{0}, N_{1}\right)$ along $N_{1}$ gives rise to a new cobordism $\left(V^{\prime}, N_{0}, N_{2}\right)$ with $N_{2}=\chi\left(N_{1}\right)=N_{1}$ surgered along the image of the attaching map. Thus by Lemma 1.5 above we have that 


$$
\begin{aligned}
M_{0} \# k_{1}\left(S^{2} \times S^{2}\right) & \# k_{2}\left(S^{2} \underset{\sim}{\times} S^{2}\right) \\
& =M=M_{1} \# k_{1}^{\prime}\left(S^{2} \times S^{2}\right) \# k_{2}^{\prime}\left(S^{2} \underset{\sim}{ } S^{2}\right)
\end{aligned}
$$

with $k_{1}+k_{2}=k_{1}^{\prime}+k_{2}^{\prime}=k$.

Furthermore, since $W$ is an $h$-cobordism $w_{2}\left(M_{0}\right)=w_{2}(M)=w_{2}\left(M_{1}\right)$, so by Lemma 1.3 we have that, for some $k$,

$$
M_{0} \# k\left(S^{2} \times S^{2}\right)=M_{1} \# k\left(S^{2} \times S^{2}\right)
$$

as desired.

COROLlary 1.6. Let $M_{1}, M_{2}$ be h-cobordant simply-connected compact smooth 4-manifolds. Then there exist integers $k_{1}, k_{2}$ such that

$$
M_{1} \# k_{1} P \# k_{2} Q=M_{2} \# k_{1} P \# k_{2} Q \text {. }
$$

Furthermore for any simply-connected such $M$ there exist integers $l_{1}$ and $l_{2}$ such that

$$
M \# l_{1} P \# l_{2} Q=a P \# b Q
$$

where $a=l_{1}+\frac{1}{2}\left(b_{2}(M)+\sigma(M)\right)$ and $b=l_{2}+\frac{1}{2}\left(b_{2}(M)-\sigma(M)\right)$.

Proof. We note that it is not difficult to check that $S^{2} \times S^{2} \# P=$ $(P \# Q) \# P=2 P \# Q$. Thus adding $\# P$ to both sides of (4) above immediately gives us result $\left(4^{\prime}\right)$. Result (5) follows by noting that any 1-connected 4-manifold $M$ will be cobordant to $\sigma(M) P$ (where we identify $-n P$ with $n Q$ ). Using Theorem 1.2 we can always obtain an $h$-cobordism

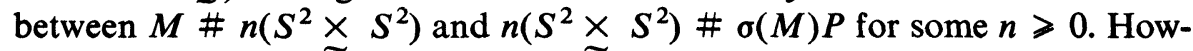
ever, $S^{2} \underset{\sim}{\times} S^{2}=\widetilde{P} \# Q$. Thus $M \# n P \# n Q$ is $h$-cobordant to $n P \# n Q \# \sigma(M) P$ and (5) follows from (4'). We note that $k_{1}$ (or $l_{1}$ ) can be taken as $k_{2}+1$ (or $\left.l_{2}+1\right)$ in ( $\left.4^{\prime}\right)$ (or (5) above).

REMARK 1. The existence of $k$ above was demonstrated in a highly nonconstructive fashion. To quote Wall, "our result is a pure existence theorem; we have obtained, even in principle, no bound whatever on the integer $k$." It is of course a question of some interest to obtain bounds on $k$. (If the $h$-cobordism conjecture were true in dimension 4 , then $k$ could of course always be taken to be zero. However its validity is of course not known, and in what follows we will discuss at length attempts to estimate $k$.)

REMARK 2. We will discuss to what extent Theorems 1.2 and 1.4 can be generalized in the non-simply-connected case in \$6.4. Roughly speaking, we can obtain generalizations for 4-manifolds $X$ with $\pi_{1}(X)=\mathbf{Z}$ or $\pi_{1}(X)=$ $\mathbf{Z}_{2 k+1}$ or for any manifold with $H_{2}\left(X, \mathbf{Z}_{2}\right)=0$ and $L_{5}\left(\pi_{1}(X)\right)=0$. However the theorems do not generalize for $\pi_{1}(X)=\mathbf{Z}_{2}$ or $\pi_{1}(X)=\mathbf{Z}^{4}$. In particular, if $X=\mathbf{R} P^{4}$ we have the following remarkable theorem of Cappell and Shaneson [CS 2]

Theorem (See ChAPTER 4), [CS 2]. There exists a compact smooth 4-manifold $X$ such that $X$ is simple homotopy equivalent to $\mathbf{R} P^{4}$ but $\mathbf{R} P^{4} \# k\left(S^{2} \times\right.$ $\left.S^{2}\right)$ is not diffeomorphic to $X \# k\left(S^{2} \times S^{2}\right)$ for any value of $k$. In fact, $\mathbf{R} P^{4} \# k\left(S^{2} \times S^{2}\right)$ is not smooth or PL $h$-cobordant or normally corbordant to $X \# k\left(S^{2} \times S^{2}\right)$ for any $k$. ( $X$ is topologically $h$-cobordant to $\mathbf{R} P^{4}$ but it is not known whether $X$ and $\mathbf{R} P^{4}$ are homeomorphic or not.) 
Using either the techniques of [CS 1] or extensions of [Nor] one can actually prove the following. (See also [Law 1], [Law 2], [Law 3] and [Q] and Chapter 6 of this paper.)

THEOREM 1.7. Suppose $\left(W^{5}, M_{0}, M_{1}\right)$ is an h-cobordism between compact 4-manifolds. Then for some $k \geqslant 0, W \# k\left(S^{2} \times S^{2} \times I\right)$ is diffeomorphic to $\left(M_{0} \# k\left(S^{2} \times S^{2}\right)\right) \times I$. In particular, $M_{0} \# k\left(S^{2} \times S^{2}\right)$ is diffeomorphic to $M_{1} \# k\left(S^{2} \times S^{2}\right)$.

REMARK 3. As we have mentioned the proof of the $h$-cobordism theorem breaks down when we have a 5-dimensional $h$-cobordism between 4-dimensional manifolds. Thus if $V^{5}$ is an $h$-cobordism between $M_{1}$ and $M_{2}$ with $\pi_{1}\left(M_{i}\right)=0$ we can not conclude that $V^{5}$ is diffeomorphic to a product. However if it is known that $V^{5}$ induces a diffeomorphism between its ends then a theorem of Barden ([Bd], [Sh 1]) does indeed let us conclude that $V^{5} \approx M_{1} \times I$. Although properly a result about 5-dimensional manifolds we will sketch a proof in $\$ 6.6$.

Returning to our main line of thought we shall call a manifold $M$ completely decomposable if $l_{1}=l_{2}=0$ in (5) above (i.e. $M=a P \# b Q$ for appropriate $P$ and $Q$ ). Now although it is not unreasonable to conjecture that $k_{1}, k_{2}$ equal 0 in $\left(4^{\prime}\right)$ for all $h$-cobordant simply-connected $M_{i}$, a similar conjecture in (5) about the $l_{i}$ (i.e., that all such simply-connected 4-manifolds are completely decomposable) is clearly false. In fact any 4-dimensional spin manifold (such as $S^{2} \times S^{2}$ or $k\left(S^{2} \times S^{2}\right)$ ) can never be completely decomposable. In fact if $M$ is Spin it can never even be homotopy equivalent to a connected sum of the form $a P \# b Q$. To see this clearly, we digress to discuss the classification of bilinear forms. As the bilinear forms arising from the cup-product on compact 4-manifolds are symmetric and unimodular we restrict our attention solely to such forms.

1.2 Four-manifolds and symmetric forms. Let $M$ be an orientable closed 4-manifold. Then $M$ has a symmetric cup-product pairing

$$
H^{2}(M ; \mathbf{Z}) \times H^{2}(M ; \mathbf{Z}) \stackrel{L_{M}}{\rightarrow} H^{4}(M ; \mathbf{Z}) .
$$

Furthermore if $\tilde{H}^{2}(M ; \mathbf{Z}) \approx H^{2}(M ; \mathbf{Z}) /$ Torsion then we shall let $\tilde{H}^{2}(M ; \mathbf{Z})$ $\times \tilde{H}^{2}(M ; \mathbf{Z}) \stackrel{L_{M}}{\rightarrow} \mathbf{Z}$ denote the induced form. By Poincare duality it is a nonsingular form and we can therefore represent $L_{M}$ by a symmetric unimodular integral matrix $A_{M}$. Clearly isomorphism classes of the $L_{M}$ are in 1-to-1 correspondence with congruence classes of the matrices $A_{M}$.

If $M$ has a boundary $\partial M \neq \varnothing$ one still has a pairing

$$
H^{2}(M, \partial M ; \mathbf{Z}) \times H^{2}(M ; \mathbf{Z}) \rightarrow H^{4}(M, \partial M ; \mathbf{Z}) \approx \mathbf{Z}
$$

and using the homomorphism $H^{2}(M, \partial M ; \mathbf{Z}) \stackrel{j}{\rightarrow} H^{2}(M ; Z)$ one again obtains a bilinear form $H^{2}(M ; \mathbf{Z}) \times H^{2}(M ; \mathbf{Z}) \rightarrow \mathbf{Z}$ which will be nonsingular on $H^{2}(M ; Z) /$ torsion. We continue to use the terminology $\bar{L}_{M}, L_{M}$ for the pairings in this case also. We shall discuss the classification of the $L_{M}$ following [MH], [Ser 1], and [Ser 2].

Thus we shall say a symmetric bilinear form $L: H \times H \rightarrow \mathbf{Z}$ is of type II 
(or is even) if its associated quadratic form $Q_{L}$ takes on only even values (which is equivalent to some associated matrix $A_{L}$ having only even entries on its diagonal). Otherwise we say $L$ is of type $I$. Now every $Q_{L}$ can be diagonalized over the rationals and thus we define $\sigma(L)$, the signature of $L$, as the signature of $Q_{L}$ over the rationals (= number of positive terms $p$ minus the number of negative terms $n$ in the diagonalization of $Q_{L}$ ). Now rank $Q_{L}=\operatorname{dim} H$ (since $L$ is assumed to be nonsingular) and if this in turn equals $|\sigma(L)|$ we say $L$ is definite. If $|\sigma(L)|<\operatorname{dim} H, L$ is said to be indefinite. An element $x \in H$ is called primitive if there exists a $y \in H$ such that $L(x, y)=$ \pm 1 . If $x \in H$ is such that $L(x, y)=Q_{L}(y)(\bmod 2)$ for all $y \in H$, then $x$ is said to be characteristic, otherwise it is ordinary. It is easy to see that characteristic elements always exist and are unique (modulo $2 H$ ). (We use the nonsingularity of $Q$ here.)

We shall also have occasion to consider quadratic forms over free $\mathbf{Z}_{2}$-modules $H$. Thus suppose $\mu: H \rightarrow \mathbf{Z}_{2}$ is a quadratic form with associated symmetric bilinear form $\lambda: H \times H \rightarrow \mathbf{Z}_{2}$ (the relationship between $\lambda$ and $\mu$ is given by $\lambda(x, y)=\mu(x+y)-\mu(x)-\mu(y)$, of course). Suppose $\mu$ is nonsingular. Then there exists a basis $a_{i}, b_{i}, i=1, \ldots, n$, for $H$ such that $\lambda\left(a_{i}, a_{j}\right)=$ $\lambda\left(b_{i}, b_{j}\right)=0$ and $\lambda\left(a_{i}, b_{j}\right)=\delta_{i j}([\mathrm{Br} 1$, p. 54]). We define the Arf invariant $c(\mu) \in \mathbf{Z}_{2}$ by $c(\mu)=\sum_{i=1}^{n} q\left(a_{i}\right) q\left(b_{i}\right)(\bmod 2)$. Then one can show: See $[\operatorname{Br} 1$, p. 55]

(1) $c(\mu)$ is independent of the choice of basis of $H$.

(2) If $\left(H_{1}, \mu_{1}\right),\left(H_{2}, \mu_{2}\right)$ are quadratic $Z_{2}$-inner-product spaces, then $\left(H_{1}, \mu_{1}\right)$ is isomorphic to $\left(H_{2}, \mu_{2}\right)$ if and only if $\operatorname{rk} H_{1}=\operatorname{rk} H_{2}$ and $c\left(\mu_{1}\right)=c\left(\mu_{2}\right)$.

(3) Let $H=\mathbf{Z}_{2}(a) \oplus \mathbf{Z}_{2}(b)$ and define quadratic forms $q_{0}, q_{1}$ on $H$ by $q_{0}(a)=q_{0}(b)=0, q_{1}(a)=q_{1}(b)=1, q_{0}(a+b)=q_{1}(a+b)=1$ (note that for both $q_{0}, q_{1}$ we have that the associated $\lambda_{0}, \lambda_{1}$ satisfy $\lambda_{0}(a, b)=\lambda_{1}(a, b)=$ 1 and $\left.\lambda_{0}(a, a)=\lambda_{0}(b, b)=\lambda_{1}(a, a)=\lambda_{1}(b, b)=0\right)$. Then if $(H, \mu)$ is a quadratic $\mathbf{Z}_{2}$-inner-product space we have $\operatorname{dim}_{\mathbf{Z}_{2}} H=2 m$ for some $m>0$, and

(a) $c(\mu)=0$ implies $(H, \mu)$ is equivalent to $\left(H, m q_{0}\right)$,

(b) $c(\mu) \neq 0$ implies $(H, \mu)$ is equivalent to $\left(H, q_{1}+(m-1) q_{0}\right)$ where we note that $2 q_{0}$ is always equivalent to $2 q_{1}$.

For closed manifolds $M^{4}$, we take $H=\tilde{H}^{2}(M ; Z)$ and $L$ the cup-product form. Then $x \in H$ is characteristic if and only if its mod 2 reduction is the second Steifel-Whitney class $w_{2}(M)$. It is then clear that $L$ is of type II if and only if $w_{2}(M)=0$, that is if and only if $M$ is spin. (We note that in our situation spin is equivalent to almost parallelizable. (See $\$ 4.1$ for a definition.) To see this we note that if $\mathcal{T}: M \rightarrow$ BSO is the map corresponding to the tangent bundle, then the obstruction to homotoping $\mathcal{T}$ to a constant lies in $H^{i}\left(M, \pi_{i}(\mathrm{BSO})\right)$. Now $w_{2}^{*}(M)=\sigma^{*}\left(\omega^{2}\right)$ where $\omega^{2} \in H^{2}(\mathrm{BSO}) \approx \pi_{2}(\mathrm{BSO}) \approx$ $\mathbf{Z}_{2}$ is the generator. Thus $w_{2}(M)=0 \Rightarrow \mathscr{T}_{M} \mid K_{M}^{3}$ is trivial (where $K_{M}^{3}$ is the 3-skeleton of $M$ ). Thus $\mathscr{T}_{M} \mid M-\{\mathrm{pt}\} \sim 0$ and so $M$ is almost parallelizable.)

As we have just noted spin manifolds have forms of type II and thus can never be isomorphic to connected sums of $P$ 's and $Q$ 's which are of type $I$. To formulate a more realistic conjecture about decomposability we continue our discussion of bilinear and quadratic forms.

We first define a few canonical forms. We let $\pm I$ be the form with matrix 
representatives $\pm\langle 1\rangle, U$ the form represented by $\left(\begin{array}{ll}0 & 1 \\ 1 & 0\end{array}\right)$ and $E_{8}$ the form with matrix representative

$$
\left(\begin{array}{llllllll}
2 & 1 & 0 & 0 & 0 & 0 & 0 & 0 \\
1 & 2 & 1 & 0 & 0 & 0 & 0 & 0 \\
0 & 1 & 2 & 1 & 0 & 0 & 0 & 0 \\
0 & 0 & 1 & 2 & 1 & 0 & 0 & 0 \\
0 & 0 & 0 & 1 & 2 & 1 & 0 & 1 \\
0 & 0 & 0 & 0 & 1 & 2 & 1 & 0 \\
0 & 0 & 0 & 0 & 0 & 1 & 2 & 0 \\
0 & 0 & 0 & 0 & 1 & 0 & 0 & 2
\end{array}\right) .
$$

By a misuse of notation we shall let $\pm I, U, E_{8}$ represent the corresponding inner-product spaces.

(More generally if $n=4 k$ let $\Gamma_{n}$ be the subspace of $\mathbf{R}^{n}$ generated by all linear combinations of the vectors $\pm e_{i} \pm e_{j}, \frac{1}{2} \sum_{i=1}^{4 k} e_{i}$. Let $V_{1}, \ldots, V_{n}$ be any vector basis of $\Gamma_{n}$ and let $M_{n}$ be the corresponding intersection form. Then $E_{n}$ is the bilinear form with matrix representative congruent to $M_{n}$. We note that a straightforward calculation shows that $M_{n}$ is unimodular and definite; and of type II if and only if $k$ is even.)

We note that $I, U, E_{8}$ are all unimodular and $I$ and $E_{8}$ are definite. We then have the following classification theorems.

Lemma 1.8 (VAN DER BLIJ). SeE [MH, p. 24]. Let $L$ be a symmetric unimodular form on $H \times H$. Then there exists $u \in H$ such that $u$ is characteristic and $u \cdot u=\sigma(L)(\bmod 8)$.

In particular if $L$ is of type II, $\sigma(L) \equiv 0(\bmod 8)$.

If $L$ is indefinite we obtain

THEOREM 1.9 (SERRE) [Ser 1] [Ser 2]. Let $L$ be an indefinite unimodular symmetric form of rank $r=p+n$ and signature $\sigma=p-n(p, n \geqslant 0)$

(1) if $L$ is of type I then $L \approx p I \oplus n(-I)$,

(2) if $L$ is of type II then $L \approx a E_{8} \oplus b U$ where $a=\frac{1}{8} \sigma$ and $b=\frac{1}{2}(r-|\sigma|)$ ( $a \in \mathbf{Z}$ by the lemma given above).

We will not attempt a sketch of Serre's proof of the above theorem but refer the reader to [MH] for both Serre's classification theorem and van der Blij's lemma. If $L$ is definite then a complete classification as above is not known. For what partial results exists see [MH] and [Ser 1].

Now suppose that $L$ is a symmetric unimodular form. When will there exist a compact 1-connected manifold $M$ whose cup-product $L_{M}$ is isomorphic to $L$ ?

Clearly we must have $\operatorname{dim}(M)=4 k$. Now if we allow $k>1$ then we have a great deal of flexibility in finding the appropriate $M^{4 k}$. In fact if $L$ is a form corresponding to a 'weighted tree' as described in [Hirz 2] then $L$ can always be realized by a $4 k$-dimensional PL-manifold for every $k>1$. More particularly for every $k \geqslant 2$, there exists a $(2 k-1)$-connected closed $4 k$-dimensional PL spin-manifold $M_{1}^{4 k}$ with $\sigma\left(M_{1}^{4 k}\right)=8$ and even more explicitly every form $E_{8 n}$ can be realized by a $(2 k-1)$-connected closed $4 k$-dimensional PL-manifold $M_{n}^{4 k}$ for every $k \geqslant 2$. To construct $M_{1}^{4 k}$ we can proceed as 
follows: Let $Y_{k} \subset C^{2 k+1}$ be the locus $\sum_{i=1}^{2 k-1} Z_{i}^{2}+Z_{2 k}^{3}+Z_{2 k+1}^{5}=1$. Let $B$ be a large ball about the origin such that $\partial B$ intersects $Y_{k}$ transversely and let $V_{k}=\overline{Y_{k} \cap B}$. Then $\partial V_{k}$ is a homology sphere, and if $k>1$ it is also simply connected. Therefore by the generalized Poincaré conjecture [Sm] $H_{k}=\partial V_{k}$ is PL-isomorphic to $S^{4 k-1}$ and thus the cone on $H_{k}, c H_{k}$, is PL-isomorphic to $D^{4 k}$. Letting $M_{1}^{4 k}=V_{k} \cup c H_{k}$ we obtain a PL-manifold of dimension $4 k$, which is $(2 k-1)$-connected and has an associated quadratic form isomorphic to $E_{8}$. (We note that 'coning off' is a piecewise-linear operation and thus as defined $M_{1}^{4 k}$ is not a smooth manifold. We shall see later in this chapter that the $M_{1}^{4 k}$ can not admit any smooth structure and thus furnish us with examples of nonsmoothable PL-manifolds.)

Now if $k=1$ it is possible to show that $\partial V_{1}$ is, in fact, the Poincare homology sphere $P$ with fundamental group $\tilde{A}_{5}=$ binary icosahedral group. Attempting to build an $M_{1}^{4}=V_{1} \cup c P$ gives us a 1-connected Poincaré complex realizing the form $E_{8}$. The complex has a nonmanifold point at the vertex $\nu$ of $c P$. However recent results of Edwards and Cannon [Ed 1], [Can] show that for any homology 3-sphere $H, c H \times R^{n}$ is a topological manifold for all $n \geqslant 1$. Thus although $M_{1}^{4}$ is not a manifold, $M_{1}^{4} \times R^{n}$ always will be! We will use these results later to exhibit compact topological manifolds which admit no PL-structure!

Whether or not there exists a 4-dimensional simply-connected closed manifold $M$ with $L_{M} \approx E_{8}$ is an unresolved question. However the following remarkable theorem of Rohlin [R 1] shows that were such a manifold to exist it could not have a PL (or smooth) structure.

TheOREM 1.10 (Rohlin). Let $M^{4}$ be a compact oriented PL-manifold with $w_{2}\left(M^{4}\right)=0$. Then $\sigma\left(M^{4}\right) \equiv 0(\bmod 16)$ !

(Thus the 4-dimensional case presents a definite anomaly from the PL point of view.) We postpone a discussion of the proof of Rohlin's theorem to $\$ 1.4$ and limit ourselves here to a discussion of its consequences for our classification problem.

We first note that Rohlin's theorem implies that if $M^{4}$ is a spin manifold with indefinite intersection form then $L_{M}=a^{\prime}\left(E_{8} \oplus E_{8}\right) \oplus b U$, where $a^{\prime}=$ $a / 2, a, b$ as in Theorem 1.9. So subject to this new restriction we still ask which forms can be realized. We note that if $M=\mathbf{C} P^{2}=P$ then $L_{P}=I$ and similarly $L_{Q}=-I$ and so any form, indefinite of type $I$, can be realized as a connected sum of $P$ 's and $Q$ 's. Now if $M=S^{2} \times S^{2}$ then $L_{M}=U$. So suppose $L$ is of type II and indefinite, with $\sigma(L) / 16 \in \mathbf{Z}$. Then were there to exist a simply-connected PL spin-manifold $V$ with $b_{2}(V)=\sigma(V)=16$ we could assert that $a^{\prime} V \# b\left(S^{2} \times S^{2}\right)$ realizes $L$, where $a^{\prime}=\sigma(L) / 16$ and $b=\frac{1}{2}(\operatorname{rk} L-|\sigma(L)|)$. However no such manifold $V$ is known to exist! In fact no closed 4-manifolds (TOP or PL) with definite intersection forms other than $\# P$ or $\# Q$ are known to exist!

The simplest known 4-manifold with $|\sigma(M)|=16$ known has $b_{2}(M)=22$ and $\sigma(M)=-16$. The Kummer or $K 3$ surface [Spr 2], [K 3] is such a manifold and by a theorem of Kodaira [K 1] is diffeomorphic to the projective algebraic variety $V_{4} \subset \mathbf{C} P^{3}\left(V_{4}\right.$ the nonsingular quadratic hyper- 
surface in $\mathbf{C} P^{3}$ with equation $\Sigma_{i=0}^{3} Z_{i}^{4}=0$, where $\left(Z_{0}: Z_{1}: Z_{2}: Z_{3}\right)$ are homogeneous coordinates in $\mathbf{C} P^{3}$ ).

Thus the most that can be said about realizing type II intersection forms is that if $L$ is such an indefinite form with $a^{\prime}, b$ as above, and if in addition $b>3\left|a^{\prime}\right|$, then $M_{L}=a^{\prime}\left(-V_{4}\right) \# b^{\prime}\left(S^{2} \times S^{2}\right)$ realizes $L$, where $b^{\prime}=b-$ $3\left|a^{\prime}\right|$. Thus any simply-connected smooth 4-manifold $M$ with form $L$ is $h$-cobordant to $M_{L}$. However if $M$ is an arbitrary simply-connected closed 4-manifold then either $M \# P$ or $(-M) \# P$ has an indefinite form of type $I$. Thus either $M \# P$ or $(-M) \# P$ is $h$-cobordant to a completely decomposable manifold. (We say $M$ is completely decomposable if it is diffeomorphic to a connected sum of $P$ 's and $Q$ 's.) If $M \# P$ is completely decomposable we shall say that $M$ is almost completely decomposable (ACD).

We then conjecture that

Conjecture I. Let $M^{4}$ be a simply-connected compact PL manifold. Then either $M$ or $-M$ is almost completely decomposable.

1.3 Almost complete decomposability and analytic surfaces. Before tackling this conjecture we recall a few lessons from the classification theory of compact orientable 2-manifolds.

We recall that every such 2-manifold $F_{g}$ is topologically just a connected sum $\#_{i=1}^{q}\left(T^{2}\right)$; of 2-tori (where the empty sum $F_{0}$ is defined to be $S^{2}$ ). Now although this classification can be done in a purely topological fashion, one of the beautiful things about orientable 2-manifold is that every such manifold is diffeomorphic to a nonsingular algebraic curve in $\mathbf{C} P^{3}$. (In fact, every such manifold is diffeomorphic to a hypersurface in either $S^{2} \times S^{2}$ or $P \# Q$.)

Thus the topology of orientable 2-manifolds can be studied by algebraic or analytic methods and in fact it can be shown that if $M$ is a compact complex 1-dimensional manifold, then $M=F_{h}$; where $h$ is the complex dimension of the space of holomorphic 1-forms on $M$.

Now if $n=4$ it is of course not the case that all 4-manifolds, even all simply-connected 4-manifolds, admit complex structures. For example neither $S^{4}$, nor $Q$, nor $S^{2} \times S^{2} \# S^{2} \times S^{2}$ admit such structures. However the compact algebraic surfaces do provide a wide variety of examples of 4-manifolds. (It can in fact be shown ([Shaf 1]) that given any finite group $G$ there exists a compact algebraic surface $V_{G}$ with $\pi_{1}\left(V_{G}\right)=G$.) Furthermore as a consequence of a theorem of Yau ([Y]; see also [Reid], [My]) all simply-connected analytic surfaces other than $\mathbf{C} P^{2}$ have indefinite intersection forms. It is therefore reasonable to conjecture that

CONJECTURE $I^{\prime}$. Let $V$ be a simply-connected compact analytic surface. Then $V$ is almost completely decomposable.

The bulk of the next chapter will analyze what progress has been made on Conjecture $I^{\prime}$ and related questions. Before beginning our discussion we would like to (1) indicate just how badly the $h$-cobordism conjecture could conceivably fail in dimension 4, and (2) sketch some proofs of Rohlin's theorem and its generalizations.

To this end let $\Lambda=\left\{(p, m, n) \in \mathbf{Z}^{3} \mid p \geqslant 0, m \geqslant 1, n \geqslant 1\right.$ and g.c.d. $(m, n)=1\}$.

Let $\Lambda^{\prime}=\{(p, m, n) \in \Lambda \mid p$ is odd and $m+n$ is even $\}$ and let $\Lambda^{\prime \prime}=\Lambda-$ $\Lambda^{\prime}$. 
Lastly for every $p \geqslant 0$ let $\Lambda_{p}=\{(m, n) \mid(p, m, n) \in \Lambda\} ; \Lambda_{p}^{\prime}=\Lambda_{p} \cap \Lambda^{\prime} ;$ $\Lambda_{p}^{\prime \prime}=\Lambda_{p} \cap \Lambda^{\prime \prime}$. Then we have

Proposition 1.11. Let $p$ be an integer $\geqslant 0$. Let $\sim_{h}$ denote "is $h$-cobordant to". Then

(1) If $p$ is odd then for every $(m, n) \in \Lambda_{p}^{\prime}$ there exists a compact algebraic surface

$$
V_{(p, m, n)} \sim_{h}((p+1) / 2) V_{4} \#((p-1) / 2)\left(S^{2} \times S^{2}\right)=W_{p}^{\text {II }}
$$

such that if $\{m, n\} \neq\left\{m^{\prime}, n^{\prime}\right\}$ then it is not known whether or not $V_{(p, m, n)}=$ $V_{\left(p, m^{\prime}, n^{\prime}\right)}$.

(2) If $p$ is $>1$ then for every $(m, n) \in \Lambda_{p}^{\prime \prime}$ there exists a compact algebraic surface

$$
V_{(p, m, n)} \sim_{h}(2 p+1) P \#(10 p+9) Q=W_{p}^{\mathrm{I}}
$$

such that if $\{m, n\} \neq\left\{m^{\prime}, n^{\prime}\right\}$ then it is not known whether or not $V_{(p, m, n)}=$ $V_{\left(p, m^{\prime}, n^{\prime}\right)}$

(3) If $p=0$ and $m \geqslant 2$ and $n \geqslant 2$ then the conclusions of (2) above hold unchanged. If however either $m=1$ or $n=1$ then in fact $V_{(0, m, 1)}=V_{(0,1, n)}=$ $P \# 9 Q=W_{0}^{\mathrm{I}}$.

We note that $\sigma\left(V_{(p, m, n)}\right)=-(8 p+8), b_{2}\left(V_{(p, m, n)}\right)=12 p+10$ and $V_{(p, m, n)}$ is spin, in case (1) and not spin in cases (2) and (3). All of the above surfaces are in fact algebraic elliptic surfaces and will be discussed at length in the next chapter.

We note further that by Theorem 4, there need not exist any finite $k$ such that for fixed $p$ every $V_{(p, m, n)}$ with $(m, n) \in \Lambda_{p}^{\prime}$ (resp. $\left.\Lambda_{p}^{\prime \prime}\right)$ satisfies $V_{(p, m, n)} \# k\left(S^{2} \times S^{2}\right)=W_{p}^{\mathrm{II}} \# k\left(S^{2} \times S^{2}\right)\left(\right.$ resp. $V_{(p, m, n)} \# k\left(S^{2} \times S^{2}\right)=$ $\left.W_{p}^{1} \# k\left(S^{2} \times S^{2}\right)\right)$.

Thus in dimension 4, the higher-dimensional surgery classification of $[\mathbf{B r} \mathbf{1}]$, [N] can fail completely and there might in fact be infinite families of $h$-cobordant simply-connected manifolds (all diffeomorphic to projective algebraic varieties), no two of which are diffeomorphic.

1.4 Rohlin's theorem. Let $M^{4}$ be a closed, orientable PL 4-manifold. Let $a \cdot b$ denote the intersection pairing (dual to the cup-product pairing) on $H_{2}(M ; Z) /$ torsion $\times H_{2}(M ; Z) /$ torsion to $Z$. As before we say that $\omega \in$ $H_{2}(M ; Z) /$ torsion is characteristic if its mod 2 reduction is Poincaré dual to the second Steifel-Whitney class $w_{2}(M)$ of $M$. Now if $\omega \in H_{2}(M ; Z) /$ torsion then one can always represent $\omega$ by an orientable 2-manifold $K$. When $K$ is characteristic (i.e., represents a characteristic homology class) it is possible to associate a quadratic form $\tilde{q}: H_{1}\left(K ; \mathbf{Z}_{2}\right) \rightarrow \mathbf{Z}_{2}$ to $K$ and an Arf invariant $\phi(M, K) \in \mathbf{Z}_{2}$ to the pair $(M, K)($ See $[\mathbf{F K}]$.

We can then state the following generalization of Rohlin's theorem.

Theorem 1.12 (Generalized Rohlin AS IN [FK], [CS 2], [R2], [R3]). Let $M^{4}$ be a closed, orientable PL 4-manifold. Let $K$ be an orientable 2-manifold embedded in $M$ and representing a characteristic homology class. Then $(K \cdot K-$ $\sigma(M)) / 8 \equiv \phi(M, K) \bmod 2$. 
Corollary 1.13 (See Theorem 1, [KM 1]). If $K$ above is a 2-sphere then $K \cdot K-\sigma(M) \equiv 0(\bmod 16)$.

Corollary 1.14 (Rohlin's TheOREM). If $w_{2}(M)=0$ then $\sigma(M) \equiv 0$ $(\bmod 16)$.

The full proof of Theorem 1.12 can be found in [FK]. We will give a rather detailed outline of Matsumoto's proof [Mt 1] of Theorem 1.12 in the case when $H_{1}(M)=0$. (The major simplification in the case when $H_{1}(M)=0$ arises in the definition of $\tilde{q}$.) We will also recall the homotopy theoretic proof of Rohlin's theorem as found in [MK]. We note that the basic germ of the more geometric version of the proof of Rohlin's theorem is due to Andrew Casson [Cas 1]. (A similar geometric construction showing how Corollary 1.13 implies Theorem 1.12 can also be found in [CS 2].)

Firstly assuming Theorem 1.12 we see that if $K$ is a 2-sphere $H_{1}(K)=0$ and so $\phi(M, K)=0$. Corollary 1.13 then follows by checking that if $w_{2}(M)=0$ we can take $K=\varnothing$ so that $K \cdot K=\phi(M, K)=0$.

Now assume that $M^{4}$ is compact, PL with (or without) boundary $\partial M^{4}$ and that $H_{1}\left(M^{4}\right)=0$. Let $K$ be an orientable characteristic surface properly embedded in $M^{4}$ with $\partial K=\varnothing$ or $\partial K=S^{1}$. (Note that if $\partial K \neq \varnothing$ we have $K \in H_{2}(M, \partial M)$ and $K$ characteristic then means that $K \cdot X+X \cdot X=0$ $(\bmod 2)$ for $X \in H_{2}(M)$, where $K \cdot X$ is the pairing on $H_{2}(M, \partial M) \times$ $H_{2}(M)$.) We define $\tilde{q}: H_{1}\left(K ; \mathbf{Z}_{2}\right) \rightarrow \mathbf{Z}_{2}$ as follows: Suppose $u \in H_{1}\left(K ; \mathbf{Z}_{2}\right)$ is represented by an embedded circle $\delta \subset K$. Using $H_{1}(M)=0$ we can find an orientable embedded surface $\Delta \subset M$ such that $\partial \Delta=\delta$ and $\operatorname{int}(\Delta)$ intersects $K$ transversely.

Then set $\tilde{q}_{K}(\delta)=\theta(\Delta)+\operatorname{card}(\operatorname{Int} \Delta \cap K)(\bmod 2)$, where $\theta(\Delta) \in \pi_{1}\left(\mathrm{SO}_{2}\right)$ $\approx \mathbf{Z}$ is the obstruction to extending the normal 1-field of $\delta \subset K$ over $\Delta$ (i.e., $\theta(\Delta)=\Delta \cdot \Delta^{\prime}$ ', where $\Delta^{\prime}$ is obtained by 'deforming' $\Delta$ slightly, using the normal 1-field of $\delta$, such that $\partial \Delta \cap \partial \Delta^{\prime}=\varnothing$ ).

It is then straightforward to verify that

$$
\tilde{q}\left(\delta_{1}+\delta_{2}\right)=\tilde{q}\left(\delta_{1}\right)+\tilde{q}\left(\delta_{2}\right)+\delta_{1} \cdot \delta_{2}
$$

( $\cdot$ is intersection of 1-cycles on $K(\bmod 2)) \cdot \tilde{q}$ is thus a well-defined quadratic function and has an associated Arf invariant $\operatorname{Arf}(\tilde{q})=\sum_{i=1}^{q} \tilde{q}\left(\alpha_{i}\right) \cdot \tilde{q}\left(\beta_{i}\right)$ where the $\alpha_{i}, \beta_{i}, i=1, \ldots, g$, are any symplectic basis of $H_{1}\left(K ; \mathbf{Z}_{2}\right)$ (i.e., $\left.\alpha_{i} \cdot \alpha_{j}=\beta_{i} \cdot \beta_{j}=0, \alpha_{i} \cdot \beta_{j}=-\beta_{j} \cdot \alpha_{i}=\delta_{i j}\right)$.

We define $\operatorname{Arf}(K)=\operatorname{Arf} \tilde{q}_{K}$ and verify that

(i) If $\partial K=\varnothing, \operatorname{Arf}(K)$ depends only on $[K] \in H_{2}(M)$.

(ii) If $\partial K=S^{1}, \operatorname{Arf}(K)$ depends on $[K, \partial K] \in H_{2}(M, \partial M)$ and on the isotopy class of $\partial K \rightarrow \partial M$.

We now demonstrate that

$$
([K] \cdot[K]-\sigma(M)) / 8=\operatorname{Arf}(K) \quad(\bmod 2) .
$$

First we recall that if $T$ is the 2-torus $S^{1} \times S^{1}$ then letting $(1,0),(0,1)$ denote the obvious basis of $H_{1}\left(T, \mathbf{Z}_{2}\right)$, we have that $(p, q) \in H_{1}(T)$ can be represented by an embedded circle if and only if $p=q=0$ or g.c.d. $(p, q)=$ 1. If g.c.d. $(p, q)=1$ we let $T_{p, q}$ denote any embedded circle representing $(p, q)$ where we think of $T \hookrightarrow S^{3}$ by the standard embedding. $T_{p, q}$ is called a 
torus knot of type $(p, q)$ and we also recall [RIf] that $T_{p, q}$ is ambient isotopic to $T_{p^{\prime}, q^{\prime}}$ in $S^{3}$ if and only if $(p, q)= \pm\left(p^{\prime}, q^{\prime}\right)$ or $(p, q)= \pm\left(q^{\prime}, p^{\prime}\right)$.

Now if $L$ is any tame knot sitting in $S^{3}$ we can by (ii) above define $\operatorname{Arf}(L)$ as $\operatorname{Arf}(F)$, for any surface $F \subset D^{4}$ with $\partial F=L \subset \partial D^{4}=S^{3}$. We then can show by direct calculation that if $p$ is odd and $q$ even,

$$
\operatorname{Arf}\left(T_{p, q}\right)=\operatorname{Arf}\left(T_{q, p}\right) \equiv\left(1-p^{2}\right) / 8 \quad(\bmod 2) .
$$

Now to demonstrate the validity of $(*)$ we first note that we can surger $M$ without affecting (*) until it is 1-connected. Then by Corollary 1.4 there exist integers $l_{1}, l_{2}, a, b$ such that (5) holds. (I.e., $M \# l_{1} P \# l_{2} Q=a P \# b Q$.) Let $\eta \in H_{2}(P)=\mathbf{Z}, \bar{\eta} \in H_{2}(Q) \approx \mathbf{Z}$ be the generators. We note that $\eta(\bar{\eta})$ are characteristic elements of $H_{2}(P)$ and that both are representable by embedded spheres. Furthermore since $\eta \cdot \eta=+1(\bar{\eta} \cdot \bar{\eta}=-1)$ it is immediately evident that the pair $(P, \eta)[(Q, \bar{\eta})]$ satisfies the equality (*). However all the quantities in (*) are additive with respect to connected sum. Thus utilizing (5) it is easy to see that (*) is true in general if and only if it holds for characteristic elements of $\mathbf{C} P^{2}$. Now $s \eta \in H_{2}\left(\mathbf{C} P^{2}\right)$ is characteristic if and only if $s$ is odd. Let $C_{s}: X_{2}^{s}+X_{1}^{s-1} X_{0}=0$ be the algebraic curve representing $s \eta$. Then $C_{s}$ is homeomorphic to $S^{2}$ and smoothly embedded except at $(0: 0: 1)$, where it has a cusp singularity. Then letting $B$ be a small 4-ball centered at the origin it is clear that $\operatorname{Arf}\left(C_{s}\right)=\operatorname{Arf}\left(\partial B \cap C_{s}\right)$ and $\left(\partial B \cap C_{s}\right)$ is isotopic to $T(s, s-1), s$ odd. But $\operatorname{Arf}\left(C_{s}\right) \equiv\left(1-s^{2}\right) / 8(\bmod 2)$ which is precisely what $(s \eta \cdot s \eta-\sigma(P)) / 8$ is $(\bmod 2)$. Thus $(*)$ is demonstrated.

The original proof of Rohlin's theorem was more homotopy theoretic. In outline we proceed as follows: Recall the homomorphism $J: \pi_{k-1}\left(\mathrm{SO}_{m}\right) \rightarrow$ $\pi_{m+k-1}\left(S^{m}\right)$ defined by

$$
[f] \in \pi_{k-1}\left(\mathrm{SO}_{m}\right) \rightarrow J[f] \in \pi_{m+k-1}\left(S^{m}\right)
$$

where $J(f): S^{m+k-1} \rightarrow S^{m}$ is the map induced from $\left(f\right.$, id): $S^{k-1} \times S^{m-1} \rightarrow$ $S^{m-1}$ given by $(f, \mathrm{id})(x, y)=f(x) \cdot y$ by passing to

$$
S^{k-1} * S^{m-1}=S^{m+k-1} \stackrel{J(f)}{\rightarrow} \sum S^{m-1}=S^{m} .
$$

Then using Steenrod algebra calculations we can show that $\pi_{3}\left(\mathrm{SO}_{m}\right) \rightarrow$ $\pi_{m+3}\left(S^{m}\right)$, with $m$ large, is onto. But $\pi_{3}\left(\mathrm{SO}_{m}\right) \approx \mathbf{Z}$ and $\pi_{m+3}\left(S^{m}\right) \approx \mathbf{Z}_{24}$. Thus for $x \in \pi_{3}\left(\mathrm{SO}_{m}\right), J x=0 \Rightarrow x \equiv 0(\bmod 24)$.

Now suppose $M^{4}$ is a closed smooth 4-manifold with $w_{2}(M)=0$. Then as we mentioned previously $M$ is almost parallelizable. Thus the stable principal normal bundle $\nu^{s}$ is trivial on $M^{4}-\{\mathrm{pt}\}$ and the obstruction to extending a cross section $f$ of $\nu^{s} \mid M^{4}-\{\mathrm{pt}\}$ to a cross section of $\nu^{s}$ defines an element $\vartheta(\nu, f) \in H^{4}\left(M^{4}, \pi_{3}\left(\mathrm{SO}_{m}\right)\right) \approx \pi_{3}\left(\mathrm{SO}_{m}\right)=\mathbf{Z}$. We can then verify that $J \theta(\nu, f)=0$. Thus $\theta(\nu, f)$ is divisible by 24 . Now we can relate $\theta(\nu, f)$ to the Pontryagin numbers of $M$ and we find that $p_{1}(M)= \pm 2 \vartheta(\nu, f)$. Thus $p_{1}(M) \equiv 0(\bmod 48)$. But by Hirzebruch's identity [Hirz 1] we have $p_{1}(M)=$ $3 \sigma(M)$ so we obtain $\sigma(M) \equiv 0(\bmod 16)$ as desired.

The argument relating almost parallelizability to $J \theta=0$ generalizes straightforwardly to higher dimensions. In fact the following can be shown. 
THEOREM 1.15. Let $M^{4 n}$ be an almost parallelizable smooth 4-manifold. Then $\sigma\left(M^{4 n}\right) / 8$ is divisible by $I_{n}=2^{2 n-2}\left(2^{2 n-1}-1\right) a_{n}$ (numerator $\left.B_{n} / 4 n\right)$, where $B_{n}$ is the nth Bernoulli number and

$$
a_{n}= \begin{cases}1 & \text { if } n \text { is even, } \\ 2 & \text { if } n \text { is odd. }\end{cases}
$$

(We note: $B_{1}=\frac{1}{6}, B_{2}=\frac{1}{30}, B_{3}=\frac{1}{42}, B_{4}=\frac{1}{30}, B_{5}=\frac{5}{66}, B_{6}=\frac{691}{2730}, B_{7}=\frac{7}{6}$, $B_{8}=\frac{3617}{510}$.)

Then if the numerator $\left(B_{n} / 4 n\right)=N_{n}$ we have $N_{1}=N_{2}=N_{3}=N_{4}=N_{5}$ $=1, N_{6}=691, N_{7}=1, N_{8}=3617$. Thus if $n=1$ we get that $\sigma\left(M^{4}\right) / 8$ is divisible by 2 which is Rohlin's theorem again.

If $n>1$ we see that Theorem 1.15 provides us with necessary conditions for an almost parallelizable manifold to have a smooth structure. In particular all the PL-manifolds $M_{1}^{4 k}$ with $\sigma\left(M_{1}^{4 k}\right)=8$ will not admit smooth structures. Thus the generalized Rohlin theorem shows us that there exist PL manifolds which can not be smoothed!

Rohlin's theorem also provides us with a tool to show that there exist TOP manifolds (of dimension $\geqslant 5$ ) which admit no PL structures. In our discussion of the construction of the $M_{1}^{4 k}$ we mentioned that although $M_{1}^{4}$ is not a manifold, the double suspension theorem of [Ed 1], [Can] shows that $M_{1}^{4} \times$ $R^{l}$ and thus $M_{1}^{4} \times S^{1}$ will, in fact, be a topological manifold. Now for $k>0$ let $Q_{4+k}=M_{1}^{4} \times S^{k}$. We claim $Q_{4+k}$ is then a compact topological $(4+k)$ manifold which admits no PL structure whatsoever. To see this suppose that $Q_{4+k}$ did admit a PL structure. Then by PL transversality we could define a map $f: Q_{4+k} \rightarrow S^{k}$ such that $f^{-1}(*)$ is a PL manifold $X_{4}$. But then we would have $\sigma\left(X_{4}\right)=\sigma\left(M_{1}^{4}\right)=8$ which contradicts Rohlin's theorem! Thus no PL structure can exist on $Q_{4+k}$.

As mentioned previously the existence of a closed, compact TOP 4-manifold with form realizing $E_{8}$ is unknown. In fact in dimension 4 there are no known examples of a compact TOP manifold which is not smoothable.

The anomalous nature of the PL category in dimension 4 as exhibited by Rohlin's theorem provides us not only with a tool by which to create non-PL manifolds but serves also to show that the PL $s$-cobordism theorem must be false for cobordism of dimension either 4 or 5 (or both). We shall discuss this application further in Chapter 6.

1.5 Aspherical 4-manifolds. At the other end of the spectrum from the simply-connected compact 4-manifolds lie the aspherical ones. We recall that a connected compact manifold $M$ is aspherical if its universal covering manifold $M$ is contractible. Thus $M$ is a $K(\pi, 1)$ with $\pi=\pi_{1}(M)$.

By a well-known theorem of homotopy theory the $K(\pi, 1)$ 's are unique up to homotopy equivalence. The basic question we must then ask is: Suppose $f$ : $\boldsymbol{M} \rightarrow M^{1}$ is a homotopy equivalence between aspherical 4-manifolds. When can $f$ be deformed to a diffeomorphism? This in turn is equivalent to the following problem.

Suppose $M$ and $N$ are aspherical 4-manifolds with isomorphic fundamental groups. Are they diffeomorphic? It turns out (see [CR 1], [CR 2], [CR 3]) that the methods used to study this problem are not unexpectedly essentially 
independent of the fact that the manifolds in question are of dimension 4. Instead general methods have been developed [CR 3] which apply simultaneously to all such finite-dimensional manifolds. As a result of this we do not devote any time to explaining the methods involved but refer the reader to [CR 3]. As a contrast to the general 4-dimensional situation we will mention some of the theorems arising in the study of such manifolds.

We first note that the most obvious examples of aspherical 4-manifolds arise as quotients of Euclidian 4-space $\mathbf{R}^{n}$ or hyperbolic 4-space $\mathbf{H}^{4}$ by proper discontinuous groups of isometrics. As a result of the rigidity theorems of Mostow (see [Most]) we obtain that such closed hyperbolic 4-manifolds are determined up to isomorphism by their fundamental groups. Similar results hold for quotients of $\mathbf{R}^{4}$ by isometries.

Another major class of aspherical 4-manifolds is given by the aspherical Seifert fibrations. We recall that $(X, \phi)$ is a (locally injective) Seifert fibration if and only if

(1) $\phi: N \rightarrow \operatorname{Aut}\left(Z^{k}\right)$ is a homomorphism of a discrete group $N$ into $\operatorname{Aut}\left(Z^{k}\right)=\operatorname{Aut}\left(T^{k}, 1\right)(k \geqslant 1)$, where $T^{k}=\left(S^{1}\right)^{k}$ is the $k$-torus.

(2) There exists an epimorphism $\lambda: \pi_{1}\left(X, X_{0}\right) \rightarrow N \rightarrow 1$ such that on the covering space $X^{\prime}$ corresponding to the kernel of $\lambda$, there exists a structure of a principal $k$-torus bundle which is compatible with the group of covering transformations $N$ on $X^{\prime}$. That is for all $t \in T^{k}, x^{\prime} \in X^{\prime}, \alpha \in N$ we must have

$$
\left(t x^{\prime}\right) \alpha=\left(\phi\left(\alpha^{-1}\right)(t)\right)\left(x^{\prime} \alpha\right)
$$

(3) $X^{\prime} / T^{k}=W$ is simply connected.

We note that induced on $W$ is an action $(W, N)$ of $N$ so that the corresponding natural maps

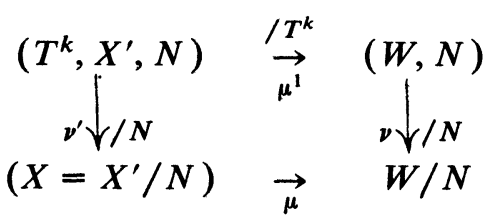

commute.

We call the mapping $\mu: X \rightarrow W / N$ a Seifert fibering.

We may construct Seifert fiberings with given $\phi$ as follows.

Let $N$ be a discrete group with $\phi: N \rightarrow \mathrm{GL}(k, \mathbf{Z})$ a homomorphism and suppose $N$ acts in a proper discontinuous fashion on the simply-connected space $W$. Set $X^{\prime}=T^{k} \times W$. Then in [CR 1], [CR 2] it is shown that there is a 1-to-1 correspondence between actions of $N$ on $X^{\prime}$ which are compatible with the obvious toral actions and elements of $H_{\phi}^{2}\left(N, \mathbf{Z}^{k}\right)$. For each such free action $T^{k} \circ N$ form $X=X^{\prime} / N$ and let $\mu: X \rightarrow W / N$ be the obvious map. We have thus constructed a Seifert fiber space corresponding to $\phi$. If $W$ is contractible then as in [CR 3] it can be shown that $X$ is a $K(\pi, 1)$.

Now suppose $W=\mathcal{F}^{2}=$ upper half-plane with the Poincare metric and $N$ is a discrete subgroup of $\operatorname{PSL}(2, \mathbf{R})$ with $W / N$ compact. We then call $X$ a Fuchsian Seifert fibering. We then have 
TheOREM 1.16 [CR 2], [CR 3]. Let $(X, \phi),\left(X^{\prime}, \phi^{\prime}\right)$ be Fuchsian Seifert fibrations with $k=2$.

Then $X$ is diffeomorphic to $X^{\prime}$ if and only if $\pi_{1}(X)$ is isomorphic to $\pi_{1}\left(X^{\prime}\right)$.

We note that the class of Fuchsian Seifert fibrations include most of the 4-manifolds with torus or circle action discussed in ([OR 1], [OR 2], [Pao 1], [Pao 2]) as well as all of the aspherical elliptic surfaces of ([K 1], [K 2]). Restricting attention to such surfaces we obtain: (See [K 1] or Chapter 2, for definitions.)

COROLlaRY 1.17 [CR 2], [CR 3]. Let $V, V^{\prime}$ be elliptic surfaces with no degenerate fibers and suppose neither $V$ or $V^{\prime}$ have coverings of the form $S^{1} \times S^{3}$ or $S^{1} \times S^{1} \times S^{2}$. Then $V$ is diffeomorphic to $V^{\prime}$ if and only if $\pi_{1}(V)$ is isomorphic to $\pi_{1}\left(V^{\prime}\right)$.

Note. It can be shown that the elliptic surface $V$ is aspherical if and only if it has no degenerate fibers and no coverings of the form $S^{1} \times S^{3}$ or $T^{2} \times S^{2}$.

\section{CHAPTER 2. COMPLEX SURFACES}

2.1 Classifying complex surfaces. In the last section we conjectured that all simply-connected complex manifolds of real dimension 4 were almost completely decomposable. To begin our study of such manifolds we recall the simply-connected version of the Kodaira-Enriques classification theorem for analytic surfaces. (Henceforth by analytic or complex surfaces we shall mean nonsingular compact complex spaces of pure complex dimension 2.)

TheOREM 2.1 (Kodaira, ENRiQues), [K 1], [K 2]. Let $M$ be a simply-connected analytic surface. Then $M$ is diffeomorphic to a projective algebraic variety which is either (1) rational or (2) elliptic or (3) of general type.

Thus when considering simply-connected complex manifolds we can analyze them category by category. We therefore recall the definitions of the various categories and introduce some numerical invariants to distinguish them. Before doing this we simplify the topological nature of our classification process by introducing minimal models.

We recall [K 1] that if $V$ is an analytic surface and $p \in V$ there exists a new analytic surface $\tilde{V}$ and a map $\sigma: \tilde{V} \rightarrow V$ such that if $\sigma^{-1}(p)=L$, then $L$ is an embedded $\mathbf{C} P^{1}\left(=S^{2}\right)$ in $\tilde{V}$ with $L \cdot L=-1$ and $\sigma \mid \tilde{V}-L$ is a biholomorphic map between $\tilde{V}-L$ and $V-p . \tilde{V}$ is said to be obtained by blowing $V$ up at the point $p$ or by a $\sigma$-process on $V$ at $p$. Topologically we can build $\tilde{V}$ by replacing a disc $D^{4}$ about $p$ in $V$ with a Hopf disc-bundle $H$ (whose boundary is also $S^{3}$ ) arranging orientations so that $L^{2}=-1$.

Noting that $H \cup D^{4}$ is just $Q$ we see that $\tilde{V}=V \# Q$. This decomposition immediately shows us that $b_{2}(\tilde{V})=b_{2}(V)+1, \sigma(\tilde{V})=\sigma(V)-1$ and we can calculate that $c_{1}^{2}(\tilde{V})=c_{1}^{2}(V)-1$. We shall sometimes denote such a $\tilde{V}$ by $\sigma V$ and if we perform $n \sigma$-processes successively we shall denote their end product by $\sigma_{n} V$. The $\sigma$-operation preserves analytic structure so that $V \# Q$ has analytic structure if $V$ does. Topologically we can form $V \# P$ in the same local fashion as we formed $V \# Q$. This has the effect of replacing a 
point $p \in V$ by an embedded 2-sphere $L$ with $L \cdot L=+1$ and we denote the resultant 4-manifold by $\bar{\sigma} V$ and call the process a $\bar{\sigma}$-process. Even if $V$ is a complex manifold it is not difficult to deduce that not only can $V \# P$ not have a complex structure but it does not even admit an almost complex structure [Pit].

Now what about going the other way. Suppose we knew that the complex manifold $V$ admitted an analytically embedded 2 -sphere $L$ with $L^{2}=-1$. Could we conclude that $V=\sigma_{p} W$ for some analytic $W$ with $p \in W$ ? (That is, if we know $V=W \# Q$ topologically when can we say it equals $W \# Q$ analytically?)

The positive answer to the above question is provided by the Castelnuevo criterion which guarantees the existence of the desired analytic surface $W$.

We call $L$ as above an exceptional curve and note that any analytic manifold $V$ is biholomorphic to $\sigma_{n} V^{\prime}=V^{\prime} \# n Q$ for some $n \geqslant 0$ and some $V^{\prime}$ without any exceptional curves. We call such a $V^{\prime}$ a minimal surface and note that for purposes of topological classification it suffices to consider minimal surfaces.

We now define the various categories of surfaces.

We recall that any surface $S$ has associated to it a field of meromorphic functions $\mathfrak{T}_{S}$ analogous to the field of meromorphic functions associated to a Riemann surface (algebraic curve). (See, for example, [K 1], [Zar 1].)

Then

Definition 2.2. (1) $S$ is a rational surface if and only if $\mathfrak{T}_{S}$ is isomorphic to $\mathbf{C}(x, y)=$ the field of rational functions in the variables $x$ and $y$.

(2) $S$ is an elliptic surface if and only if for some Riemann surface $R$, there exists a holomorphic map $f: S \rightarrow R$ such that $f^{-1}(x)$ is a nonsingular elliptic curve (i.e., a 2-torus $T^{2}$ ) for all but finitely many $x \in R$. There is an equivalent definition in terms of the fields $\mathfrak{\pi}_{S}, \mathfrak{T}_{R}$.

(3) $S$ is of general type (in the simply-connected case) if it is neither rational nor elliptic.

The above definitions prove a bit tedious to check in individual cases. We thus provide some numerical criteria for distinguishing classes by introducing some analytic invariants of the various classes.

Let $V$ be a complex surface and denote its tangent bundle by $T(V)$ and its cotangent bundle by $T^{*}(V)$. Recall that any complex surface has a sheaf of germs of complex functions (its structure sheaf) associated to it and denote this by $\theta_{V}$. Lastly if $L$ is any vector bundle over $L$ let $\theta_{V}(L)$ denote the sheaf of germs of holomorphic sections over $L$.

We now set $K=\Lambda^{2} T^{*}(V)$. $K$ is thus a complex line bundle over $V$ called the canonical line bundle.

We let $n K$ denote $K \otimes \cdots \otimes K$, i.e., the $n$-fold direct product.

Now define the geometric genus of $V$,

$$
p_{g}[V]=p_{g}=\operatorname{dim}_{\mathbf{C}} H^{0}\left(V, \vartheta_{V}(K)\right)=\operatorname{dim}_{\mathbf{C}} H^{2}\left(V, \vartheta_{V}\right)
$$

$=\mathrm{C}$-dimension of the space of holomorphic 2-forms on $V$. The plurigenii $P_{n}$ of $V$ are defined as

$$
P_{n}=\operatorname{dim}_{\mathbf{C}} H^{0}\left(V, \mathcal{O}_{V}(n K)\right) .
$$


Also associated to $V$ are its Chern classes

and

$$
c_{1}[V]=c_{1}[T(V)]=-c_{1}\left[T^{*}(V)\right]=-c(K)
$$

$$
c_{2}[V]=\chi(V)=\sum(-1)^{i} \text { rk } H_{i}(V ; \mathbf{Z}) .
$$

The basic theorems relating the analytic and topological invariants of $V$ are:

(1) The Riemann-Roch theorem, which for simply-connected surfaces reduces to the Noether equality

$$
c_{1}^{2}[V]+\chi(V)=12\left(p_{g}+1\right)
$$

or

$$
b_{2}(V)=12 p_{g}+10-c_{1}^{2}[v] .
$$

(2) The Hodge index theorem $b_{2}^{+}(V)=2 p_{g}+1$ where $b_{2}^{+}(V)=\frac{1}{2}\left(b_{2}(V)+\right.$ $\sigma(V))$ or using (1) above if $V$ is simply-connected

$$
\sigma(V)=c_{1}^{2}[V]-8\left(p_{g}+1\right)
$$

implying

$$
c_{1}^{2}[V]=2 \chi(V)+3 \sigma(V) .
$$

Recalling our discussion of the homotopy types of simply-connected 4-manifolds, we see that $V$ is thus determined up to homotopy type ( $h$-cobordism type, really) by $p_{g}(V), c_{1}^{2}[V]$ and the $\bmod 2$ reduction of $c_{1}[V]=w_{2}[V]$ which determines the type of $L_{V}$.

A numerical criterion for rationality is due to Castelnuevo. It is [K 2]:

Castelnuevo Criterion. An analytic surface $V$ is rational if and only if $b_{1}(V)=P_{2}(V)=0$.

Minimal rational surfaces have been completely classified, and if $V$ is a minimal rational surface it is diffeomorphic to either $P$ or $S^{2} \times S^{2}$. Thus we have (see [Zar 2] or [Shaf 2])

THEOREM 2.3. Let $V$ be a rational surface. Then $V$ is diffeomorphic to either $S^{2} \times S^{2}$ or $P \# n Q$ for some $n \geqslant 0$. In particular, all rational surfaces are $A C D$.

The elliptic and general type surfaces are not so easily classified but they too have numerical criteria by which they can be recognized. More particularly for simply-connected surfaces

(1) $V$ is elliptic if and only if $V^{\prime}$ is a minimal model for $V$ implies $c_{1}^{2}\left(V^{\prime}\right)=0$.

(2) $V$ is of general type iff $V^{\prime}$ is a minimal model for $V$ implies $c_{1}^{2}\left(V^{\prime}\right)>0$ and $P_{2}\left(V^{\prime}\right) \neq 0$.

There is, in addition, a theorem of Yau [Y] and Miyaoka [My] which says that for all analytic surfaces $V, c_{1}^{2}(V) \leqslant 3 \chi(V)$ with equality holding only if $V=\mathbf{C} P^{2}$. Combining this with the above equations we can deduce that:

(1) all simply-connected complex surfaces $V$ other than $\mathbf{C} P^{2}$ have indefinite $L_{V}$, and 
(2) every simply-connected complex spin manifold $V$ satisfies

$$
b=\frac{1}{2}\left(b_{2}(V)-|\sigma(V)|\right) \geqslant 3 a^{\prime}=3|\sigma(V)| / 16
$$

and thus is homotopy equivalent to $a^{\prime} V_{4} \# b^{\prime}\left(S^{2} \times S^{2}\right)\left(V_{4}\right.$ the nonsingular quartic in $\left.\mathbf{C} P^{3}\right) ; b^{\prime}=b-3 a^{\prime}$.

2.2 Decomposing surfaces. I. Rather than proceeding directly to the case of elliptic surfaces we first prove

THEOREM 2.4 (MANDELBAUM-MoishezoN) [MM 1]. Let $V_{n}$ be a nonsingular hypersurface of $\mathbf{C} P^{3}$. Then $V_{n}$ is almost completely decomposable.

Proof. We will need the following two facts.

FACT (1) Let $W_{n}$ be a hypersurface of $\mathbf{C} P^{k}(k \geqslant 3)$. Then $W_{n}$ is simplyconnected. (This is essentially Lefschetz's first theorem: see ([AF], [Wlc], or [Zar 1]).)

FACT (2) Suppose $W_{n}, W_{n}^{\prime}$ are nonsingular, degree $n$, hypersurfaces of $\mathbf{C} P^{k}$. Then $W_{n}$ is diffeomorphic to $W_{n}^{\prime}$.

Proof. One constructs a mapping $\mathbf{C} P^{k} \times \mathbf{C} P^{N(k)} \stackrel{\Phi}{\rightarrow} \mathbf{C} P^{N(k)}\left(N(k)=\left(\begin{array}{c}n+k \\ k\end{array}\right)\right.$ -1) whose fibers consist of all possible degree $n$ hypersurfaces in $\mathbf{C} P^{k}$ and checks that the critical values of $\Phi$ correspond precisely to those hypersurfaces which are singular.

We note also that if $n$ is small the topological structure of $V_{n}$ is completely determined. Thus $V_{1}=P, V_{2}=S^{2} \times S^{2}, V_{3}=P \# 6 Q$. $V_{4}$ has already been described as the 'simplest' known spin manifold of index \pm 16 . It satisfies $\sigma\left(V_{4}\right)=-16$ and $b_{2}\left(V_{4}\right)=22$. If $n \geqslant 5$, the homotopy structure of the $V_{n}$ is still known. We have $b_{2}\left(V_{n}\right)=n^{3}-4 n^{2}+6 n-2, \sigma\left(V_{n}\right)=\frac{1}{3} n(4-$ $\left.n^{2}\right)$ and $V_{n}$ is of type II if $n \equiv 0(\bmod 2)$. Thus homotopically we have

$$
\begin{gathered}
V_{2 m+1} \sim r_{m} P \# t_{m} Q, \\
r_{m}=\frac{2}{3}\left[(2 m+1)\left(2 m^{2}-4 m+3\right)\right]-1, \quad t_{m}=\frac{2}{3}(m)\left(8 m^{2}+1\right), \\
V_{2 m} \sim \alpha_{m} V_{4} \# \beta_{m} V_{2}, \\
\alpha_{m}=\frac{1}{6} m\left(m^{2}-1\right), \quad \beta_{m}=\frac{1}{6}(m-2)\left(13 m^{2}-22 m+3\right) .
\end{gathered}
$$

Whether any of the $V_{n}(n \geqslant 4)$ can be decomposed as a nontrivial connected sum homeomorphically or diffeomorphically is unknown (see [MM 1]). In terms of classification of surfaces we have that if $n \geqslant 4$ then $V_{n}$ is a nonrational minimal surface with $c_{1}^{2}\left[V_{n}\right]=n(n-4)^{2}$. Thus $V_{n}(n>4)$ is of general type while $V_{4}$ is diffeomorphically of elliptic type. As noted above $V_{1}$, $V_{2}, V_{3}$ are rational surfaces (with $V_{3}$ not a minimal model).

Our proof is now an inductive one. To relate the topologies of $V_{n}$ and $V_{n-1}$ we first embed $V_{n}$ in a family $V_{t}, t \in D^{2}$, of complex manifolds with $V_{t} \simeq V_{n}$ for $t \neq 0$ and $V_{0}$ consisting of two components $X_{1}, X_{2}$ intersecting transversely in, say, $C$. In such a case we can show that

$$
V_{n} \approx V_{t}=X_{1}-T_{1} C \cup_{\partial T} X_{2}-T_{2} C,
$$

where $T_{i} C$ are the obvious tubular neighborhoods.

We then analyze such decompositions and relate the topologies of $V_{t}$ to those of $X_{1}$ and $X_{2}$. 
Let us see how this sort of procedure works one dimension lower in the case of nonsingular algebraic plane curves (i.e. hypersurfaces of $\left.\mathbf{C} P^{2}\right)$. To analyze $X_{3} \subset \mathbf{C} P^{2}$ say, we first find a degenerating family such that $X(t)=$ $X_{3}, t \neq 0$, and $X(0)=X_{1} \cup X_{2}$ intersecting transversely in two disjoint points $p_{1}, p_{2}$. Then

$$
\begin{aligned}
X_{3} & \approx X_{1}-T\left(p_{1}, p_{2}\right) \cup X_{2}-T\left(p_{1}, p_{2}\right) \\
& \approx S^{2}-D_{1} \cup D_{2} \cup S^{2}-D_{1}^{\prime} \cup D_{2}^{\prime} \approx T^{2}
\end{aligned}
$$

since $X_{1} \approx X_{2}=S^{2}$. More generally the same procedure shows us that

$$
\begin{aligned}
X_{n} & \approx X_{n-1}-T\left(p_{1}, \ldots, p_{n-1}\right) \cup X_{1}-T\left(p_{1}, \ldots, p_{n-1}\right) \\
& \approx X_{n-1} \# X_{1} \#(n-2) T^{2} \approx\left(\sum_{1}^{(n-2)}\right) T^{2}=\frac{1}{2}(n-1)(n-2) T^{2}
\end{aligned}
$$

which shows that genus $\left(X_{n}\right)=\frac{1}{2}(n-1)(n-2)$ as expected:

Now let us return to dimension 4 and hypersurface of $\mathbf{C} P^{3}$.

We proceed as follows:

(1) We construct a system $V_{n}(t)$ of hypersurfaces of degree $n$, parametrized by $t \in D_{\varepsilon}=\{z \in \mathrm{C}|| Z \mid<\varepsilon\}$ such that $V_{n}(t) \approx V_{n}$ for $t \neq 0$ and $V_{n}(0)$ is the union of two components $X_{1}, X_{2}$ with transversal intersection $X_{1} \cap X_{2}=$ $C, C$ an algebraic curve of degree $n-1$, such that $X_{1} \approx V_{1}$ and $X_{2} \approx V_{n-1}$.

(2) We modify our system to obtain a complex 3-manifold $W$ and a holomorphic map $\Phi: W \rightarrow D_{e}$ such that $\Phi^{-1}(t)=V_{n}(t)$ for $t \neq 0$ and $\Phi^{-1}(0)$ is the union of $\sigma X_{1}, X_{2}$ with transversal intersection $\sigma X_{1} \pitchfork X_{2}=\tilde{C} \approx C$, where $\sigma X_{1}=V_{1}$ blown up at the $n(n-1)$ distinct points in $X_{1} \cap X_{2} \cap V_{n}(t)$ $=V_{1} \cap V_{n-1} \cap V_{n}$ and $X_{2} \approx V_{n-1}$.

(3) We then use a deformation theorem [MM 2] to conclude that

$$
V_{n} \approx V_{n}(t) \approx \sigma V_{1}-T_{1} \tilde{C} \cup_{f} V_{n-1}-T_{2} \tilde{C},
$$

$T_{i} \tilde{C}$ an open tubular neighborhood of $\tilde{C}$ in $X_{i}$, and $f: \partial T_{1} \rightarrow \partial T_{2}$ an appropriate identification map.

Thus far we have essentially imitated the 2-dimensional case. However in that case the tubular neighborhoods which we removed from our manifolds were 2-balls and thus the transition to connected sums was fairly easy. Here, however, the $T_{i} C$ are nontrivial 2-disc bundles over compact orientable 2-manifolds! Thus to go to the connected sum we resort to the irrational connected sum theorem of [Man 1] which says that if a simply-connected 4-manifold $V$ admits a decomposition $V \approx \sigma X_{1}-T_{1} C \cup X_{2}-T_{2} C$ where $C$ is a compact orientable 2-manifold and $\sigma X_{1}=X_{1} \# k Q, k>0$, then

$$
V \# P \approx X_{1} \# X_{2} \#(k-1) Q \# 2 g(P \# Q) \text {. }
$$

Therefore we have

$$
V_{n} \# P=V_{n-1} \# V_{1} \#[n(n-1)-1] Q \# 2 g_{C}(P \# Q)
$$

where $2 g_{C}=\frac{1}{2}(n-2)(n-3)$, and by induction

$$
V_{n} \# P=k_{n} P \# l_{n} Q
$$

where $k_{n}=\frac{1}{3} n\left(n^{2}-6 n+11\right), l_{n}=\frac{1}{3}(n-1)\left(2 n^{2}-4 n+3\right)$. Thus $V_{n}$ is always $A C D$. 
The deformation and irrational connected sum theorems we used state

Theorem 2.5 (Deformation Theorem [MM 2]). Suppose $W^{n}$ is a connected complex $n$-manifold and $\pi: W^{n} \rightarrow D$ is a proper holomorphic map onto a 2-disc about the origin in $\mathbf{C}$ whose only critical value is at $0 \in D$.

Suppose that $\pi^{-1}(0)=X_{1} \cup X_{2}$ with $X_{1} \pitchfork X_{2}=C, X_{1}, X_{2}$ being connected compact submanifolds of $W^{n}$ (of multiplicity 1 in $\pi^{-1}(0)$ ) and $C$ being the connected transversal intersection.

Then there exists a diffeomorphism $f: \partial T_{1} C \rightarrow \partial T_{2} C$ where $T_{i} C$ are (small) tubular neighborhoods of $C$ in $X_{i}$ such that, for all $t \in D-\{0\}, V_{t}$ is diffeomorphic to $\overline{X_{1}-T_{1} C} \cup{ }_{f} \overline{X_{2}-T_{2} C}$

(If $W$ is as above we refer to the $\left\{V_{t}\right\}_{t \in D}$ as a nicely degenerating family.)

Theorem 2.6 (IrRational CONNected SUM) (Mandelbaum) [Man 1]. Suppose $M_{1}, M_{2}$ are oriented compact 4-manifolds and $S_{1}, S_{2}$ are oriented genus $g$ compact 2-submanifolds of $M_{1}, M_{2}$, respectively. Let $M_{2}^{\prime} \rightarrow M_{2}$ be $M_{2}$ blown up by a $\sigma$-process at some point $p \in S_{2}$ and let $S_{2}^{\prime}=\overline{\sigma^{-1}\left(S_{2}-p\right)}$. Let $T_{1}, T_{2}$, $T_{2}^{\prime}$ be tubular neighborhoods of $S_{1}, S_{2}, S_{2}^{\prime}$ in $M_{1}, M_{2}, M_{2}^{\prime}$, respectively, and set $H_{1}=\partial T_{1}, H_{2}^{\prime}=\partial T_{2}^{\prime}$.

Suppose $\eta: H_{1} \rightarrow H_{2}^{\prime}$ is a bundle diffeomorphism of $H_{1}$ onto $H_{2}^{\prime}$ that reverses orientation.

Let $V=\overline{M_{1}-T_{1}} \cup_{\eta} \overline{M_{2}^{\prime}-T_{2}^{\prime}}$. Then

(i) $V \# P$ is diffeomorphic to $M_{1}-T_{1}^{*} \cup_{\eta} \overline{M_{2}-T_{2}^{*}}$ where $T_{i}^{*}$ is a regular neighborhood of $S_{i}-\{2$-disc $\}$ in $M_{i}$ and $\eta^{*}$ is a diffeomorphism $\partial T_{1}^{*} \rightarrow \partial T_{2}^{*}$ induced by $\eta$.

Furthermore noting that $T_{i}^{*}$ has a wedge $K$ of $2 g 1$-spheres as a deformation retract in $M_{i}$ we also have

(ii) if $M_{2}$ is simply-connected then $V \# P \approx M_{2} \# \chi_{K}\left(M_{1}\right)$ (where $\chi_{K}\left(M_{1}\right)$ is $M_{1}$ surgered along disjointly embedded circles $e_{\alpha}, \alpha=1, \ldots, 2 g$, in $M_{1}$ such that if $K_{1}=V S_{\alpha}^{1}$ then $e_{\alpha}$ is homotopic to $S_{\alpha}^{1}$ in $M_{1}$ ) and

(iii) if $M_{2}$ is also simply-connected then $V \# P \approx M_{1} \# M_{2} \# 2 g(P \# Q)$.

Before discussing these theorems we go back to steps (1) and (2) of our program.

Thus for $t \in \mathbf{C}$ let

$$
Y_{n}^{N}(t)=\left\{x \in \mathbf{C} P^{N} \mid t \sum X_{i}^{n}+\left(\sum X_{i}^{n-1}\right)\left(\sum X_{i}\right)=0\right\} .
$$

Then it is readily verifiable that $Y_{n}^{N}(t)$ indeed has the properties required of the system whose existence was needed in step (1). The question then is how to make the transition from (1) to (2). For this transition step we shall need one additional proposition.

We introduce some more terminology. Thus suppose $W \subset \mathbf{C} P^{N}(z)$ is a compact complex manifold and $Y_{0}, \ldots, Y_{m}$ are hypersurfaces of $\mathbf{C} P^{N}$ of degree $r$ with defining equations $h_{i}(z)=0, i=0, \ldots, m$. For $t \in \mathbf{C} P^{m}$ let $H_{t}(z)=\Sigma t_{i} h_{i}(z)$ and let $Y_{t}$ be the hypersurface given by $H_{t}(z)=0$. Let $W_{t}$ be the $W \cap Y_{t}$. Then we shall call the collection $\left\{W_{t}\right\}$ a (linear) system of hypersurfaces (hypersurface sections) of $W$. (If $m=1$ we shall refer to the system as a pencil.) Thus for example $Y_{n}^{N}(t)$ is a pencil of hypersurfaces of 
$C P^{N}$ (more precisely, $Y_{n}^{N}(t)$ is the restriction to $\mathrm{C}$ (obtained by setting $t_{m}=1$ ) of a pencil).

Now suppose $W_{t}$ is a pencil such that

(1) $W_{t}$ is nonsingular for $t$ sufficiently small but nonzero, and

(2) $W_{0}$ is the union of two nonsingular components $X_{1}, X_{2}$ of multiplicity one such that

$$
\begin{aligned}
& X_{1} \approx W \cap\left\{\text { hypersurface of } \mathbf{C} P^{N} \text { of degree } r-l\right\}, \\
& X_{2} \approx W \cap\left\{\text { hypersurface of } \mathbf{C} P^{N} \text { of degree } l\right\},
\end{aligned}
$$

and $X_{1}$ intersects $X_{2}$ transversely, and

(3) for $t$ sufficiently small, $t \neq 0, V_{t}$ intersects $X_{1}, X_{2}$ and $X_{1} \cap X_{2}$ transversely (we say in such a case that $V_{t}, X_{1}, X_{2}$ intersect normally). We shall then call $W_{t}$ a nicely degenerating pencil of hypersurfaces. We have

PROPOSITION 2.7. Let $W_{t}$ be a nicely degenerating pencil of hypersurfaces of the compact complex manifold $W^{3} \subset \mathbf{C} P^{N}$. Then there exists a complex manifold $X$ and a proper surjective holomorphic map $X \stackrel{\Phi}{\rightarrow} D_{\varepsilon}$ (some $\left.\varepsilon>0\right)$ such that $\Phi^{-1}(t) \approx W_{t}$ is nonsingular for $t \in D_{e}, t \neq 0$ and $\Phi^{-1}(0)=X_{1}^{\prime} \cup X_{2}^{\prime}$ is the union of two nonsingular components of multiplicity one with $X_{1}^{\prime}$ intersecting $X_{2}^{\prime}$ transversely in $X_{1}^{\prime} \cap X_{2}^{\prime} \approx X_{1} \cap X_{2}$ and $X_{1}^{\prime} \approx X_{1}, X_{2}^{\prime} \approx \sigma X_{2}$ where $\sigma X_{2}$ is $X_{2}$ blown up at the (distinct) points in $W_{t} \cap X_{1} \cap X_{2}$.

We call the collection $\Phi^{-1}(t)=X_{t}$ a nicely degenerating family of complex manifolds (over $D_{\mathbf{e}}$ ).

Thus using our last three theorems we see that if we can find an appropriate nicely degenerating pencil in which to embed a given complex surface $V$ we can then decompose $V$ as in (4) and hopefully thereby prove $V$ is $A C D$ using induction.

For complete proofs of 2.5 and 2.7 we refer the reader to [MM 2] while a proof of 2.6 can be found in [Man 1]. We note here that the proof of 2.5 is essentially based on repeated use of transversality while 2.7 can be reduced to 2.5 by a series of 'blowing up' operations. The proof of 2.5 is based on elementary general position and surgery arguments. An elementary outline of the geometrical ideas behind the proofs of all three of these theorems can be found in [Man 4] and we therefore do not repeat it here. As an immediate consequence of these theorems we have the following.

Suppose $V_{1}, \ldots, V_{k}$ are hypersurfaces of $\mathbf{C} P^{N}(n>k)$. Let $X=V_{1}$ $\cap \cdots \cap V_{k}$ be a submanifold of $\mathbf{C} P^{k+2}$ such that for each $x \in X$ there exists a coordinate neighborhood $U \ni x$ in $\mathbf{C} P^{k+2}$ and local coordinates $Z=\left(Z_{1}, \ldots, Z_{N}\right)$ in $U$ with $Z(x)=0$ such that $V_{i} \cap U=\left\{Z \in U \mid Z_{i}=0\right\}$. We then call $X$ a (scheme-theoretic) complete intersection of hypersurfaces of $\mathbf{C} P^{N}$. Then

THEOREM 2.8. Every simply-connected complex surface which is diffeomorphic to a complete intersection of hypersurfaces in some $\mathbf{C P} P^{N}$ is almost completely decomposable.

TheOREM 2.9 (BRANCHED COVERS). Let $X \subset \mathbf{C} P^{N}$ be a compact complex surface and suppose $M \rightarrow X$ is an r-fold cyclic branched covering manifold of $X$ 
whose branch locus is homeomorphic to $X \cap H_{r}$, for some hypersurface $H_{r}$ of degree $r$ of $\mathbf{C P N}$. Then if $X$ is almost completely decomposable so is $M$.

To prove both of these results it suffices to display appropriate nicely degenerating pencils. To do this in Theorem 2.8 we first invoke some commutative algebra which enables us to replace the $V_{1}, \ldots, V_{k}$ with hypersurface $V_{1}^{\prime}, \ldots, V_{k}^{\prime}$ such that $V$, the complete intersection of $V_{1}, \ldots, V_{k}$ is diffeomorphic to the complete intersection of $V_{1}^{\prime}, \ldots, V_{k}^{\prime}$ and such that, in addition, the complete intersection of $V_{1}^{\prime}, \ldots, V_{k-1}^{\prime}$ is a complex manifold $W$. Then if $V_{k}^{\prime}$ has degree $r$ in $\mathbf{C} P^{k+2}$ we can use $Y_{r}^{k+2}(t)$ to produce the desired pencil connecting $W \cap$ \{hypersurface of degree $r-1$ \} with $V=W \cap V_{k}^{\prime}$. Then Theorems 2, 5, 6, 7 and a multiple induction conclude the proof.

To prove Theorem 2.9 we construct a projective bundle over $X$ in which $M$ embeds as a hypersurface section. We can then degenerate $M$ via a pencil with singular fiber $X \cup M_{r-1}, M_{r-1}$ an $(r-1)$-fold cyclic cover of $X$. Again an induction eventually shows that $M \# P \approx r X \# a P \# b Q$ for some $a, b$ $>0$. If $X$ is $A C D$ this completes the proof.

If $X$ is not known to be $A C D$ let $k(X)$ be the minimum integer such that $X \# k(P \# Q)$ is $A C D$ (we call $k(X)$ the resolving number of $X)$. The same reasoning as above then shows that if $M$ is $A C D$ so is any $(r+s)$-cyclic cover $M^{\prime}$ of the above type. If we keep track of the obtainable values of $a$ and $b$ in the downward induction from $M \# P$ to $r X \# a P \# b Q$ we obtain the following generalizations of Theorem 2.9.

THEOREM 2.10. Suppose $X \subset \mathbf{C P} P^{N}$ is a simply-connected complex surface and suppose $M \rightarrow X$ is an $r$-fold cyclic branched covering manifold of $X$ whose branch locus is homeomorphic to $X \cap H_{r}$ for some degree $r$ hypersurface $H_{r}$ of $C P^{N}$. Let $k=k(X)$ be the resolving number of $X$ and suppose $b=\operatorname{degree} X$ and $\mathrm{g}$ is the genus of $X \cap H_{1}, H_{1}$ a hyperplane of $\mathrm{CP} P^{N}$ intersecting $X$ transversely.

Then if either (1) $r^{3}>3 k / b$ or (2) $r^{2}>k / g, M$ is almost completely decomposable.

Corollary 2.11. Suppose $X$ is as above. Then there exists an integer $m_{0} \geqslant 0$ such that for any $m>m_{0}$ there is

(1) a hypersurface $H_{2 m} \subset \mathbf{C} P^{N}$ of degree $2 m$, intersecting $X$ transversely in an irreducible nonsingular curve $C_{m}=H_{2 m} \cap X$,

(2) a unique algebraic 2-fold covering manifold $M_{m}$ of $X$ with branch locus $C_{m}$ such that $M_{m}$ is almost completely decomposable.

This method gives us other results for which we establish some additional terminology. A field $F$ is called an algebraic function field of two variables over $\mathbf{C}$ if $\boldsymbol{F}$ is a finitely generated extension of $\mathbf{C}$ of transcendence degree two. Let $\mathcal{F}$ denote the collection of all such fields. Then for $F \in \mathcal{F}$ there exists a nonsingular algebraic surface whose field of meromorphic functions is $F$ (see [Zar 2]). We shall call any such nonsingular surface a model for $F$. It is then easy to see that given any two such models $V_{1}, V_{2}$ for $F$ their fundamental groups are isomorphic. Thus we define the fundamental group $\pi_{1}(F)$ for any $F \in \mathscr{F}$ as the fundamental group of any model $V$ for $F$. We then let $\mathscr{F}_{0}$ be the subcollection of simply-connected $F$ in $\mathscr{F}$. For $F \in \mathscr{F}_{0}$ we let $\mu(F)=$ 
$\inf \{k \mid \exists$ a model $V$ for $F$ such that $V \# k P$ is completely decomposable $\}$. Using the results of Chapter 1 it can be seen that $\mu(F)$ is finite for any $F \in \mathscr{F}_{0}$. If $F$ is a pure transcendental extension of $\mathbf{C}, \mu(F)=0$. If $\mu(F) \leqslant 1$ we shall call $F$ a topologically normal field. We now need

Definition 2.12. Let $L, K \in F$. Then $L$ is a satisfactory cyclic extension of $K$ if there exist models $V_{L}, V_{K}$ for $L, K$, respectively, and a morphism $\Phi$ : $V_{L} \rightarrow V_{K}$ with discrete fibers whose ramification locus $R$ is a nonsingular hypersurface section of $V_{K}$ whose degree is a multiple of $\operatorname{deg}(\Phi)$.

We can then restate Corollary 2.11 as:

THEOREM 2.13. Let $K \in \mathscr{F}_{0}$. Then there exists a satisfactory cyclic extension $L \in \mathscr{F}_{0}$ of $K$ which is of degree 2 over $K$ and topologically normal.

In [Man 1] it is further shown that if $K$ itself is topologically normal then so is any satisfactory cyclic extension. These two results motivate a partial order in $\mathscr{F}_{0}$ defined as follows:

For $L, K \in \mathscr{F}_{0}$ we shall say that $L$ is a satisfactorily resolvable extension of $K$ if and only if there exists a finite sequence of fields $L_{0}, \ldots, L_{n}$ in $\mathscr{F}_{0}$ with $L_{0}=K, L_{i+1}$ a satisfactory cyclic extension of $L_{i}$ and $L_{n}=L$. We write $K<L$ if $L$ is a satisfactorily resolvable extension of $K$. Then $<$ induces a partial ordering on $\mathscr{F}_{0}$. Our above results then say that in terms of this partial ordering every sufficiently "large" field $L$ is topologically normal.

Lastly we mention a purely topological counterpart of Theorem 2.13 .

THeORem 2.14. Suppose $X$ is a smooth simply-connected 4-manifold. Let $F \in H_{2}(X, Z)$ with $F^{2} \neq 0$ and $F$ divisible by some integer $m \geqslant 2$. Then there exists a smooth compact simply-connected 4-manifold $\tilde{X}$ and a map $\Phi: \tilde{X} \rightarrow X$ exhibiting $\tilde{X}$ as an $m$-fold branched cover over $X$ whose branch locus $R$ is a nonsingular representative of $F$ such that

(1) if $F^{2}>0$ then $\tilde{X} \# P$ is completely decomposable, and

(2) if $F^{2}<0$ then $\tilde{X} \# P$ is completely decomposable.

We note that both the classes of complete intersections and branched cyclic coverings contain the hypersurfaces of $\mathbf{C} P^{3}$ as special cases. For complete intersections this is obvious since a hypersurface of $\mathbf{C} P^{3}$ is a complete intersection by definition. As far as realizing hypersurfaces as branched cyclic covers we simply note that $V_{n}=\left\{x \in \mathbf{C} P^{3} \mid \sum X_{i}^{n}=0\right\}$ is realized as an $n$-fold cyclic cover of $\mathbf{C} P^{2}$ branched over $C_{n}=\left\{x \in \mathbf{C} P^{2} \mid \Sigma X_{i}^{n}=0\right\}$ by virtue of the obvious projection map $\pi: \mathbf{C} P^{3} \rightarrow \mathbf{C} P^{2}$ restricted to $V_{n}$. Fact 2 above then shows that all hypersurfaces can be so realized.

In fact an adaptation of the above then shows that every complete intersection is in fact diffeomorphically a cyclic branched cover and so Theorem 2.8 in its entirety is really a special case of Theorem 2.9. One can calculate the homotopy types of the cyclic coverings without much difficulty and obtain results such as those in the tables following. Note that if $V_{n}$ is a nonsingular hypersurface of $\mathbf{C} P^{3}$ of degree $n$ then $p_{g}\left(V_{n}\right)=\frac{1}{6}(n-1)(n-2)(n$ - 3) while if $X_{n}$ is a 2-fold branched cover of $\mathbf{C} P^{2}$ with branching curve of degree $2 n$ then $p_{g}\left(X_{n}\right)=\frac{1}{2}(n-1)(n-2)$. 
Let $X_{n}$ be a 2 -fold branched cover of $\mathbf{C} P^{2}$ with branching curve of degree $2 n$.

Then we have Table 1.

\begin{tabular}{c|c|c|c|c|c}
$n$ & $p_{g}\left(X_{n}\right)$ & $B_{2}\left(X_{n}\right)$ & $\sigma\left(X_{n}\right)$ & $C_{1}^{2}\left(X_{n}\right)$ & $\begin{array}{c}\text { Topological type } \\
\text { (if known) }\end{array}$ \\
\hline 1 & 0 & 2 & 0 & 8 & $S^{2} \times S^{2}$ \\
2 & 0 & 8 & -6 & 2 & $P \# 7 Q$ \\
3 & 1 & 22 & -16 & 0 & $V_{4}$ \\
4 & 3 & 44 & -30 & 2 & Unknown. Homotopic to \\
5 & 6 & 7 & -48 & 8 & $\begin{array}{c}7 P \text { Unknown. Homotopic to } \\
3 V_{4} \# 4 V_{2}\end{array}$ \\
$n$ & $\frac{1}{2}(n-1)(n-2)$ & $2\left(2 n^{2}-3 n+2\right)$ & $2\left(1-n^{2}\right)$ & &
\end{tabular}

TABLE 1

Let $Y_{n}=3$-fold covering of $\mathbf{C} P^{2}$ branched over a curve of degree $3 n$.

\begin{tabular}{c|c|c|c|c|c}
$n$ & $P_{g}\left(Y_{n}\right)$ & $B_{2}\left(Y_{n}\right)$ & $\sigma\left(Y_{n}\right)$ & $C_{1}^{2}\left(Y_{n}\right)$ & $\begin{array}{c}\text { Type } \\
\text { (topological) }\end{array}$ \\
\hline 1 & 0 & 7 & -5 & 3 & $P$ \# $6 Q \approx X_{3}$ \\
2 & 3 & 43 & -29 & 3 & Unknown \\
3 & 11 & 115 & -69 & 27 & Unknown \\
$n$ & $\frac{1}{2}(n-1)(5 n-4)$ & $18 n(n-1)+7$ & $3-8 n^{2}$ & $3(9+4 n(n-3))$ &
\end{tabular}

TABLE 2

Let $\Sigma_{k}$ be the analytic manifold obtained by projectivizing the complex vector bundle $\vartheta \oplus[\Theta(n)]$ on $S^{2}$. Let $C$ denote its zero section.

Let $Z_{j}^{m, k}$ be the cyclic $m$-fold cover of $\Sigma_{k}$ with nonsingular branching curve $C_{j}, C_{j}$ homologically equivalent to $m j C$ in $\Sigma_{k}$. Note that topologically

$$
\Sigma_{k}= \begin{cases}S^{2} \times S^{2} & \text { if } k \equiv 0(\bmod 2), \\ P \# Q & \text { if } k \equiv 1(\bmod 2) .\end{cases}
$$

Then we have Table 3.

\begin{tabular}{rr|c|c|c|c|c} 
& $j$ & $\chi\left(Z_{j}^{m, k}\right)$ & $\sigma\left(Z_{j}^{m, k}\right)$ & $p_{g}$ & $K^{2}$ & $g_{\Gamma}$ \\
\hline$m=2$ & 1 & $2 k+4$ & $-2 k$ & 0 & $8-2 k$ & 0 \\
& 2 & $12 k$ & $-8 k$ & $k-1$ & 0 & 1 \\
& 3 & $30 k-2$ & $-18 k$ & $3 k-2$ & $6 k-8$ & 2 \\
& 4 & $56 k-8$ & $-32 k$ & $6 k-3$ & $16 k-16$ & 3 \\
$m=3$ & $j$ & $8+2 j[(2 j-1) k-2]$ & $-2 j^{2} k$ & $1+\frac{1}{2} j(j-1) k-j$ & $2 X+3 \sigma$ & $j-1$ \\
& 1 & $12 k$ & $-8 k$ & $k-1$ & 0 & 1 \\
& 2 & $60 k-12$ & $-32 k$ & $7 k-4$ & $24(k-1)$ & 4 \\
& 3 & $144 k-24$ & $-72 k$ & $18 k-7$ & $24(3 k-2)$ & 7 \\
& $j$ & $12+6 j[(3 j-1) k-2]$ & $-8 j^{2} k$ & $2+\frac{1}{2} j[(5 j-3) k-6]$ & & $3 j-2$
\end{tabular}

$g_{\Gamma}$ is the genus of the generic curve lying over a fiber of $\Sigma_{k}$.

TABLE 3 
2.3 Decomposing elliptic surfaces. We now return to our original classification theorem for surfaces. We have described various families of surfaces which include in them surfaces of all three types, rational, elliptic and general type and have shown them all to be $A C D$. None of the families described however included all the elliptic or general type surfaces. Using the basic ideas developed so far we can now prove:

THEOREM 2.15 ([Man 2], [Msh]). Let $V$ be a simply-connected elliptic surface. Then $V$ is almost completely decomposable.

To prove Theorem 2.15 we will again have to find appropriate nicely degenerating systems and try to carry through an inductive type of proof. To do this we first discuss the Kodaira classification of elliptic surfaces.

Thus suppose $M$ is an elliptic surface, which we can assume without loss of generality to be minimal. We then have a holomorphic map $f: M \rightarrow R$ onto some Riemann surface $M . f$ will have only a finite number of critical values $a_{1}, \ldots, a_{n}$ and if $X$ is not a critical value then $f^{-1}(X)$ is a 2-torus $T^{2}$ of multiplicity 1 .

What are the possible topological configurations for the singular fibers $f^{-1}\left(a_{i}\right)$ ? There are two types of possibilities.

I. $f^{-1}\left(a_{i}\right)$ is topologically a 2-torus $T^{2}$ but has multiplicity $m \geqslant 2$ in $M$. (That is, for any $x \in f^{-1}\left(a_{1}\right)$ if $z$ is a local parameter at $a_{i} \in R$ with $z\left(a_{i}\right)=0$ then $x$ has a coordinate neighborhood $U(s, t)$ such that $f: M \rightarrow R$ takes the form $(s, t) \rightarrow z=s^{m}$ on $U$.)

II. $f^{-1}\left(a_{i}\right)$ has singular points.

Fibers of type I are called nondegenerate multiple fibers while fibers of type II are called degenerate fibers (they may in addition be multiple).

A complete description of all possible degenerate fibers can be found in [K 1]. For simplicity we shall use the following technical trick.

Lemma 2.16 ([Msh], [Man 5]). Suppose $V$ is an elliptic surface. Then there exists an elliptic surface $V^{\prime}$, diffeomorphic to $V$, and a holomorphic map $f$ : $V^{\prime} \rightarrow R$ onto a Riemann surface $R$ such that any degenerate fiber $f^{-1}(a)$ of $f:$ $V \rightarrow R$ is of multiplicity one and has only one singular point $p$, which is an ordinary double point.

(Recall that a singular point $p$ is an ordinary double point if and only if we can pick a local coordinate neighborhood $U(s, t)$ about $p$ such that $f \mid U$ has the form $f(s, t)=s^{2}+t^{2}$ (where $s(p)=t(p)=0$ ).)

We call a fibering of the above type a Kodaira fibering. We now analyze the multiple fibers in detail. To do this we must introduce the concept of a logarithmic transformation on an elliptic fibration.

Thus suppose $f: M \rightarrow R$ is an elliptic fibration and $a \in R$ is a regular value of $f$. Then topologically we have for some small disc $D$ about $a$ that $f^{-1}(D)=T^{2} \times D^{2}$. Let $C$ denote the curve $* \times \partial D^{2}$ on $f^{-1}(D)$ with induced orientation. On $\partial\left(f^{-1}(D)\right)=\partial\left(M-f^{-1}(D)\right)=T^{3}$ let $Q, H_{1}, H_{2}$ be the obvious simply closed curves generating $H_{1}\left(T^{3}\right)$ such that $Q$ is homotopic to zero in $f^{-1}(D)$. Let $L_{m}$ be a diffeomorphism of $\partial\left(f^{-1}(D)\right) \rightarrow \partial(M-$ $\left.f^{-1}(D)\right)$ which takes $C \rightarrow m Q+k_{1} H_{1}+k_{2} H_{2}$.

We call $M-f^{-1}(a) \cup_{L_{m}} f^{-1}(a)$ the logarithmic transformation $M^{*}$ of $M$ 
at $a$ and write it as $M^{*}=L_{a}(m) M$. (This is of course an abbreviation for $L_{a}\left(m, k_{1}, k_{2}\right) M$.)

A fundamental result of Kodaira then is:

LeMMA 2.17 [K 2]. Let $M^{*}=L_{a}(m) M$ be the logarithmic transformation of $M$ at $a$. Then $M^{*}$ can be given the structure of an elliptic surface with fibration $f^{*}: M^{*} \rightarrow R$ such that $f^{*} \mid M-f^{*-1}(a)$ 'coincides' with $f \mid M-f^{-1}(a)$ and on $f^{*-1}(a), f^{*}$ locally looks like the map $(\sigma, \phi) \rightarrow \sigma^{m}$. Then $f^{*-1}(a)$ is a multiple fiber of multiplicity $m$ in $M^{*}$.

Furthermore if $V^{*}$ is an arbitrary elliptic surface then $V^{*}=$ $L_{a_{1}}\left(m_{1}\right), \ldots, L_{a_{k}}\left(m_{k}\right) V$ where $V$ is an elliptic surface with no multiple fibers.

By way of example let us look at the elliptic surface $E=T^{2} \times S^{2}$ with projection $T^{2} \times S^{2} \rightarrow S^{2}$. Then by a direct computation we find that: (1) if $E^{*}=L_{a}(m) E$ then $E^{*}$ is diffeomorphic to $S^{1} \times L$ where $L$ is a lens space of type $(m, k)$ for some $k$ relatively prime to $m$, (2) if $E^{*}=L_{a_{1}}\left(m_{1}\right) L_{a_{2}}\left(m_{2}\right) E$ then $E^{*}$ is still diffeomorphic to $S^{1} \times$ lens space $L(m, k)$ for appropriate $m, k$ with g.c.d. $(m, k)=1$.

Let us now return to the simply-connected case and suppose $V^{*}$ is a simply-connected elliptic surface. Then following [K 3], [Man 2] we can show that $V^{*}=L_{a_{1}}\left(m_{1}\right) L_{a_{2}}\left(m_{2}\right) V$, where g.c.d. $\left(m_{1}, m_{2}\right)=1$ and $V$ admits a holomorphic map $f: V \rightarrow S^{2}$ such that $f$ has no multiple fibers and at least one degenerate fiber.

Furthermore if $p_{g}(V)$ is even then $V^{*}$ is homotopy equivalent to $V$ if and only if $m_{1}+m_{2}$ is even.

Now for the case of minimal simply-connected elliptic surfaces with no multiple fibers Kas has shown

TheOREM 2.18 [Kas 1], [Kas 2]. Let $V_{1}, V_{2}$ be simply-connected minimal elliptic surfaces ${ }^{2}$ without any multiple fibers. Then $V_{1}$ is diffeomorphic to $V_{2}$ if and only if $p_{g}\left(V_{1}\right)=p_{g}\left(V_{2}\right)$.

Now every minimal elliptic surface $W$ satisfies $c_{1}^{2}[V]=0$ and using Noether's theorem and the formulas of $\$ 2.1$ we see that if the elliptically minimal simply-connected surface with no multiple fibers $W$ has geometric genus $p_{g}(W)=p$ then denoting $W$ by $W_{p}$ we have

(1) if $p$ is even then $W_{p}$ is homotopy equivalent to $(2 p+1) P \#(10 p+$ 9) $Q$,

(2) if $p$ is odd then $W_{p}$ is homotopy equivalent to $((p+1) / 2) V_{4} \#((p-$ 1) $/ 2) S^{2} \times S^{2}$.

\footnotetext{
${ }^{2}$ In the theory of elliptic surfaces we say a surface $V$ is minimal elliptic if and only if it contains no exceptional curve of the first kind in any fiber. In fact, there occurs an elliptically minimal surface which is a rational surface containing nine exceptional curves. This happens if $\mathbf{C P}$ is blown up at the nine base points of a pencil $\lambda F+\mu G$ of cubic curves. The resulting surface $W_{0}$ is diffeomorphic to $P \# 9 Q$ but has no exceptional curves in any of its fibers and thus is called elliptically minimal. It can be seen that $L_{a}(m) W_{0}$ always satisfies the Castelnuevo criterion and thus is also rational and so diffeomorphic to $W_{0}$. However if g.c.d. $(m, n)=1$ and $m>1, n>1$, $L_{a_{1}}(m) L_{a_{2}}(n) W_{0}$ is not rational. It is called a Dolgacev surface and shows that Severi's Conjecture ( $V$ simply-connected and $p_{g}(V)=0 \Rightarrow V$ is rational) is false. It is not known if the Dolgacev surfaces are topologically $P \# 9 Q$.
} 
We have that if $W_{p}(m, n)=L_{a_{1}}(m) L_{a_{2}}(n) W_{p}$ with $(m, n)$ relatively prime: then (1) if $p$ is even, $W_{p}(m, n)$ is homotopy equivalent to $W_{p}$; (2) if $p$ is odd and $m+n$ even $W_{p}(m, n)$ is still homotopy equivalent to $W$. (3) if $p$ is odd and $m+n$ is odd $W_{p}(m, n)$ is homotopy equivalent to $(2 p+1) P \#(10 p+$ 9) $Q$. We note that if $p>0$ or both $m, n>1$ and $p \geqslant 0$ it is only known that $W_{p}(m, n)$ is diffeomorphic to $W_{p}\left(m^{\prime}, n^{\prime}\right)$ if $\left\{m^{\prime}, n^{\prime}\right\}=\{m, n\}$ as unordered pairs. If $\left\{m^{\prime}, n^{\prime}\right\} \neq\{m, n\}$ it is not known whether $W_{p}(m, n)$ is or is not diffeomorphic or even homeomorphic to $W_{p}\left(m^{\prime}, n^{\prime}\right)$. Thus as we mentioned in Chapter 1 (see (Proposition 1.11)) there could be infinitely many nonhomeomorphic simply-connected elliptic surfaces of the same homotopy type.

To show that all these elliptic surfaces are almost completely decomposable we begin by showing that for every $k \geqslant 1$ there exists a nicely degenerating family with nonsingular fiber $W_{k}$ and zero-fiber the disjoint union of $W_{k-1}$ and $W_{0}$ intersecting transversely in a common fiber.

(The actual existence of a $W_{k}$ for every $k>0$ can be demonstrated in a purely topological fashion as follows. Let $F_{k}$ be a Riemann surface of genus $k$ possessing an involution $i_{k}$ with exactly $2 k+2$ distinct fixed points (i.e. take the 2-fold branched cover of $S^{2}$ with $2 k+2$ branch points of order 1). Then $F_{k} / i_{k}=S^{2}$. Let $U_{k}=F_{1} \times F_{2} / i_{1} \times i_{k}$ with projection to $F_{k} / i_{k}$. Then $U_{k}$ is a complex variety with $n=8 k+8$ singular points $p_{1}, \ldots, p_{n}$. It can be shown that each point $p_{i}$ has a neighborhood $N_{i}$ with $\partial N_{i}$ a circle bundle over $S^{2}$ of type -2 (see [K 1] or [Kas 1], [Kas 2]). Then replacing $N_{i}$ by the appropriate disc bundle $T_{i}$ with $\partial T_{i}=\partial N_{i}$ we get $W_{k}=U_{k}-\cup N_{i} \cup \cup T_{i}$. Then $W_{k}$ can be shown to be the required elliptic surface.)

Thus since as we noted $W_{0}=P \# 9 Q$ we have using our previously developed equations that

$$
W_{k} \# P=W_{k-1} \# P \# 8 Q \# 2(P \# Q)=W_{k-1} \# 3 P \# 10 Q \text {. }
$$

The next step is to demonstrate that one can do logarithmic transformation simultaneously to all the members of a nicely degenerating family.

This then gives us that $W_{k}(m, n) \# P=W_{k-1}(m, n) \# 3 P \# 10 Q$ for all $k, m, n$. To complete the induction we must show that $W_{0}(m, n) \# P=$ $2 P \# 9 Q$. This is done by noting that

$$
\begin{aligned}
W_{0}(m, n) & =W_{0}-D^{2} \times T^{2} \cup L_{a}(m) L_{b}(n)\left(S^{2} \times T^{2}\right)-D^{2} \times T^{2} \\
& =W_{0}-T(C) \cup S^{1} \times L(p, q)-T(C)
\end{aligned}
$$

where $C$ is a torus in $V_{0}$ or $S^{1} \times L(p, q)$.

Then we obtain by Theorem 2.6

$$
W_{0}(m, n) \# P=\chi\left(S^{1} \times L(p, q)\right) \# P \# 8 Q,
$$

where $\chi\left(S^{1} \times L(p, q)\right)$ is surgery on $S^{1} \times L(p, q)$ along two disjoint 1-circles homotopy equivalent to generators of $H_{1}(C)$.

But then either by [FP], [Msh] or direct calculation one can verify that $\chi\left(S^{1} \times L(p, q)\right)=$ either $P \# Q$ or $S^{2} \times S^{2}$ and thus that $W_{0}(m, n)$ is $A C D$. The details of the construction of the appropriate family can be found in [Man 2].

Thus we have established that all simply-connected elliptic surfaces are 
$A C D$. Pushing these techniques a bit further one can also show:

THEOREM 2.19 [Man 4]. Let $k$ be odd and $m+n$ even with $(m, n)$ relatively prime. Then $W_{k}(m, n) \# S^{2} \times S^{2}$ is diffeomorphic to $\frac{1}{2}(k+1)\left[V_{4} \# S^{2} \times\right.$ $\left.S^{2}\right]$.

2.4 Surfaces of general type. We now turn to surfaces of general type. Here our conjecture is not yet established for all such surfaces.

The best general result known is the following theorem of Moishezon.

THEOREM 2.20 [MOISHEZON [Msh]]. Let $V$ be a compact complex surface of general type. Set

$$
\begin{aligned}
p & =\frac{1}{2}\left(b_{2}(V)+\sigma(V)\right), \quad n=\frac{1}{2}\left(b_{2}(V)-\sigma(V)\right), \\
k & =30,375 p^{3}+68,850 p^{2}+52,004 p+13,092, \\
l & =\max \left(0,\left(\left(60,750 p^{3}-141,750 p^{2}+110,265 p+28,595\right)-n\right)\right) .
\end{aligned}
$$

Then $V \# k P \# l Q$ is completely decomposable.

To prove this theorem one again uses a deformation theorem. In the past however to study $V$ we embedded it as the nonsingular fiber of a 'nicely degenerating' family and examined what simpler pieces $V$ degenerated into. In this case one reverses the procedure and realizes $V$ as the singular fiber of a family whose nonsingular fiber has known topology.

We must make use of the following embedding theorem.

THEOREM 2.21 (BOMBIERI) [Bom]. Let $V$ be a minimal nonsingular simplyconnected surface of general type. Let $N=P_{5}(V)-1$. Then there exists a holomorphic map $\Phi: V \rightarrow \mathbf{C} P^{N}$ with image $W=\Phi(V)$ and points $p_{1}, \ldots, p_{m}$ $\in W$ such that if $L_{i}=\Phi^{-1}\left(p_{i}\right)$ and $P=\left\{p_{i}\right\}, L=\cup L_{i}$ then $V-L \stackrel{\Phi}{\rightarrow} W-$ $P$ is a biholomorphic equivalence and each $p_{i}$ is a rational double point of $W$. Furthermore $\operatorname{deg} W \leqslant 45 p+36$ where $p=\frac{1}{2}\left(b_{2}(V)+\sigma(V)\right)$.

(We recall that a point $p \in V$ is a rational double point of $V$ if it is isolated and for all sufficiently small open sets $U \subset V$ containing $p$ we have $\pi_{1}(U-$ $p$ ) is finite but not trivial. (See [Durf]) for thirteen other equivalent characterizations.)

Now suppose $W \subset \mathbf{C} P^{N}$ is an irreducible algebraic surface in $\mathbf{C} P^{N}$. (Note that by a theorem of Severi [Sev 1] every nonsingular projective algebraic surface can actually be realized as a subvariety of $\mathbf{C} P^{5}$.) We can always define a generic projection $\pi: \mathbf{C} P^{N} \rightarrow \mathbf{C} P^{3}$ and analyze the new singularities introduced in $\pi(W)$. One finds that generically there are only three types of new singularities introduced.

They are defined locally by equations of the following sort

(i) $Z_{1} Z_{2}=0$ (double lines),

(ii) $Z_{1} Z_{2} Z_{3}=0$ (triplanar points),

(iii) $Z_{1}^{2}-Z_{2} Z_{3}^{2}=0$ (pinch point).

We call any singularity of the above type an ordinary singularity.

If $X$ is a complex 3-manifold and $V \subset X$ a complex 2-dimensional subvariety we say that $V$ has canonical singularities if and only if any singular 
point of $V$ is either a rational double point or ordinary. Generalizing Severi's theorem [Sev 1], Moishezon combines this with Bomberi's embedding theorem to show that every minimal nonsingular simply-connected surface of general type $V$ admits a surjective holomorphic map $\psi: V \rightarrow W \hookrightarrow \mathbf{C} P^{3}$ such that (1) $W$ has only canonical singularities and $V-\psi^{-1}(S) \stackrel{\psi}{\rightarrow} W-S$ is biholomorphic, where $S \subset W$ is the singular locus of $W$, and (2) the locus $S^{*} \subset S$ of ordinary singularities of $W$ is an irreducible curve. Since $\psi$ is obtained by a combination of a projection and the map $\Phi$ of Theorem 2.21 we have that $\operatorname{deg} W$ is still at most $45 p+36$.

The following type of deformation theorem is then used.

Proposition 2.22 [MoIshezon [Msh]]. Let $W^{3} \subset \mathbf{C} P^{N}$ be a complex manifold and suppose $F, G$ are degree $r$ hypersurfaces of $\mathbf{C} P^{N}$. Let $W_{t}=\left(t_{0} F+\right.$ $\left.t_{1} G\right) \cap W$ be a pencil of hypersurfaces on $W$ and suppose $V_{1}=F \cap W$ is nonsingular and $V_{0}=G \cap W$ has canonical singularities such that the locus $S^{*}$ of all ordinary singularities is an irreducible complex curve of genus $g$. Suppose also that $V_{0}$ and $V_{1}$ intersect normally in $W$.

Then if $\bar{V}$ is the minimal resolution of $V_{0}$ we have that if $\pi_{1}(\bar{V})=\pi_{1}\left(V_{1}\right)=$ $0, S^{*} \cdot V_{1}=b>0$ and $\rho>0$ is the number of pinch points of $V_{0}$ and $\nu>0$ is the number of triplanar points, then $V_{1} \# P$ is diffeomorphic to $\bar{V} \#(2 \nu+\rho+$ $2 g-1) P \#(\nu+2 \rho+b+2 g-2) Q$.

Notes 1 . We recall [Lf] that $\Phi: V \rightarrow W_{0}$ is called a minimal resolution if (1) $V$ is nonsingular and (2) $S=$ singular locus of $W$ then $V-\Phi^{-1}(S) \stackrel{\Phi}{\rightarrow} W-$ $S$ is biholomorphic and (3) if $V^{\prime} \stackrel{\Phi^{\prime}}{\rightarrow} W$ is any other map having properties (1) and (2) then $\Phi^{\prime}$ factors through $V$ uniquely: (In fact there exists a 'blowing down map' $V^{\prime} \stackrel{\rho}{\rightarrow} V$ such that $\rho$ is the composition of a finite number $n$ of $\sigma$ processes and topologically $V^{\prime}=V \# n Q$ ).

2. By the normal intersection of $V_{0}$ and $V_{1}$ we mean in this context that for all $x \in V_{1} \cap V_{0}$ there exists a local complex coordinate system $\left(Z_{1}, Z_{2}, Z_{3}\right)$ on $W$ with center $x$ such that in some neighborhood $U_{x}$ of $x$ in $W, V_{1}$ is defined by the equation $Z_{3}=0$ and $V_{0}$ is defined either by the equation $Z_{1}=0$ (if $V_{0}$ is nonsingular at $X$ ) or by $Z_{1} Z_{2}=0$ (otherwise).

The proof of Theorem 2.20 now proceeds as follows

Let $V$ be a simply-connected surface of general type. We can without loss of generality suppose that $V$ is minimal. Then we can find a hypersurface $W$ of $\mathbf{C} P^{3}$ of degree $r \leqslant 45 p+36\left(p=\frac{1}{2}\left(b_{2}(V)+\sigma(V)\right)\right)$ such that $W$ has canonical singularities and its locus of ordinary singularities is an irreducible curve and $V$ is its minimal resolution. Furthermore we can then always find a nonsingular hypersurface $V_{r}$ intersecting $W$ normally.

Thus we find that $V \# k^{\prime} P \# l^{\prime} Q$ is diffeomorphic to $V_{r} \# P$ by Proposition 2.22 where

$$
\begin{aligned}
k^{\prime} & =2 \nu+\rho+2 g-1=b^{+}\left(V_{r}\right)-b^{+}(V)+1, \\
l^{\prime} & =\nu+2 \rho+b+2 g-2=b^{-}\left(V_{r}\right)-b^{-}(V)
\end{aligned}
$$

where $b^{+}(M)$, resp. $b^{-}(M)$, equals $\frac{1}{2}\left(b_{2}(M)+\sigma(M)\right)$, resp. $\frac{1}{2}\left(b_{2}(M)-\right.$ $\sigma(M))$. 
But we have shown in Theorem 2.4 that $V_{r}$ is $A C D$ and so

$$
\begin{aligned}
V_{r} \# P & =k(r) P \# l(r) Q ; \\
k(r) & =\frac{1}{3} r\left(r^{2}-6 r+11\right), l(r)=\frac{1}{3}(r-1)\left(2 r^{2}-4 r+3\right) .
\end{aligned}
$$

Thus $k^{\prime}=k(r)-p$ and $l^{\prime}=l(r)-b^{-}(V)$. Then in particular $k^{\prime}<k(45 p+$ $36)$ and $l^{\prime}<l(45 p+36)$ and so the theorem follows.

Although these estimates give us a tractable bound on resolving numbers of complex surfaces we are still a long way from showing that all such surfaces are $A C D$. All progress in resolving Conjecture $\mathrm{I}^{\prime}$ has been made by considering families of surfaces which could be explicitly constructed. To quote Moishezon [Msh], 'the "theoretical" Theorem 2.20 gives much weaker results than our "empirical knowledge". The interesting question is, how far can we move with such 'empirical achievements' in more general classes of simplyconnected algebraic surfaces.'

\section{CHAPTER 3. 4-MANIFOLDS AND THE CALCULUS OF LINKS}

3.1 Framed links and the Kirby calculus. Having considered the question of decomposing algebraic surfaces via a combination of techniques from algebraic geometry and topology we discuss a more strictly topological method to obtain information about 4-manifolds. We recall that every PL-manifold admits a handlebody decomposition [RS, Chapter 6]. Thus to show that two manifolds are isomorphic we might try to show that they admit isomorphic decompositions. Kirby [Kirb 1] has developed a 'calculus' to manipulate handle decompositions of 4-manifolds which can be used to prove that two such manifolds are isomorphic. (See also [FR] and [Sá].)

We recall that if $M^{4}$ is a connected 4-manifold then we can always decompose $M^{4}$ as

$$
M^{4}=H^{0} \cup \cup H_{i}^{1} \cup \cup H_{j}^{2} \cup \cup H_{j}^{3} \cup \bigcup_{k=1}^{n} H_{k}^{4}
$$

where if $\partial M^{4}=\varnothing$ we can assume $n=1$ and if $\partial M^{4} \neq \varnothing$ we may assume $n=0$.

Each $p$-handle $H_{i}^{p}=D^{p} \times D^{4-p}$ has an attaching map $f_{i}$ on $S^{p-1} \times D^{4-p}$ to $M^{4} \cup$ all handles preceding $H_{i}^{p}$ \}, with $f_{i}$ an embedding, and the isomorphism class of our handlebody decomposition is determined by the isotopy classes of the $f_{i}$ [RS, p. 71]. What are the various attaching maps we must worry about in the case of 4-manifolds?

The attaching maps for 1-handles are maps $f: S^{0} \times D^{3} \rightarrow \partial W$ ( $W$ will symbolize $H^{0} \cup$ \{other handles\}). Essentially then we are just singling out neighborhoods of pairs of points as places where we will attach a 1-handle. All such $f$ are clearly isotopic since $W$ is connected and $\operatorname{dim} W>1$.

We can thus write the attaching map of 1-handles down in the form
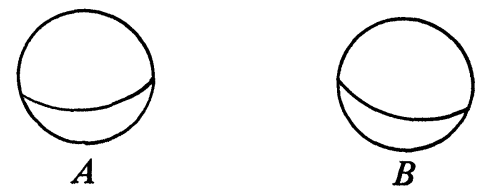
in $S^{3}=\partial H^{0}$ and our 2-handle will be an identification of the balls $A$ and $B$ in the above picture. [Note that $D^{4} \cup \cup{ }_{i=1}^{r} H_{i}{ }^{1}$ is simply $\xi_{i=1}^{r} S^{1} \times D^{3}$ with boundary $\#_{i=1}^{r} S^{1} \times S^{2}$.]

Now let us consider 2-handles. Then our attaching maps $f: S^{1} \times D^{2} \rightarrow \partial W$ are precisely framings on embedded $S^{1}$ 's in $\partial W$. Now up to isotopy such framings are classified by $\pi_{1}\left(\mathrm{SO}^{2}\right) \simeq \mathbf{Z}$ so an attaching map of a 2-handle can be regarded as an embedded $S^{1}$ (i.e. a knot) with an integer attached to it representing the framing.

More generally, since by Principle 1 of Chapter 1 we can simultaneously attach all the handles to $\partial H^{0}=S^{3}$ we make the following definition.

Definition 3.1 (Tentative). Let $M$ be an oriented 3-manifold. $L$ is a framed link in $M$ if and only if $L$ is a finite disjoint collection of smoothly embedded circles, $\gamma_{1}, \ldots, \gamma_{r}$, (knotted or unknotted), with an integer $n_{i}$ associated with each $\gamma_{i}$. (Geometrically $n_{i}$ means that the attaching map $f$ : $S^{1} \times D^{2} \rightarrow S^{3}$ with $f\left(S^{1} \times 0\right)=\gamma_{i}$ associated to $\left(\gamma_{i}, n_{i}\right)$ is precisely one such that, for any $x \in D^{2}-\{0\}, f\left(S^{1} \times\{x\}\right)$ has linking number $n_{i}$ with $\gamma_{i}$. This means that the disc $D^{2}$ is twisted $n$-times in a right-handed direction as we traverse $\gamma_{i}$ ) (We call $f\left(S^{1} \times\{x\}\right)$ a $n_{i}$-parallel curve for $\gamma_{i}$ )

If $M$ is the boundary of an oriented 4-manifold $V$ and $M$ has the induced orientation we shall let $V_{L}$ denote the manifold obtained by adding handles to $V$ along $M$ via the recipe given by the framed link $L$. We shall denote $\partial V_{L}$ by $\chi_{L}(M)$. Note that the construction of $V_{L}$ depends only on the orientation of $V$ and not on orientations of components of $L$.

EXAMPLE 1.

$$
\text { Some examples if } M=S^{3}
$$

Link $L$

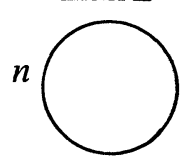

$$
\chi_{L}(M)=
$$

Lens space $L(n, 1)$

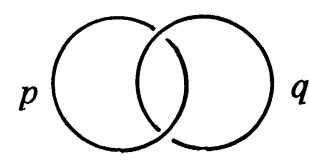

$$
L(p q-1, p)=L(p q-1, q)
$$

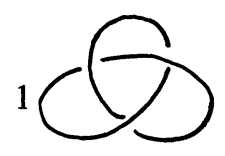

$$
\begin{aligned}
& \text { Dodecahedral space } P=S^{3} / G \\
& G=\text { binary dodecahedral group }
\end{aligned}
$$

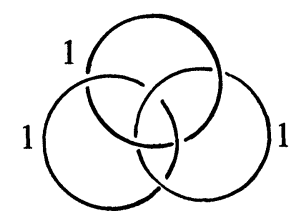


EXAMPLE 2.

Some examples of $B_{L}\left(B=B^{4}\right.$ with $\left.\partial B^{4}=S^{3}\right)$

(5)

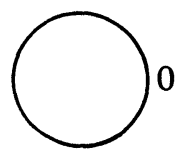

$$
S^{2} \times D^{2}
$$

(6)

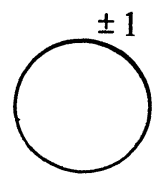

$\pm C P^{2}-B^{4}$

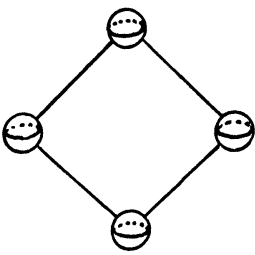

$$
T^{2} \times D^{2}
$$

(7)

$$
0 \bigcirc 1 \quad P \# Q-B^{4}
$$

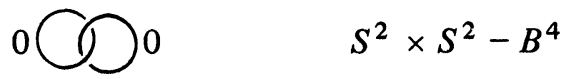

Now suppose $L, L^{\prime}$ are framed links in $S^{3}$. The framed link $L+L^{\prime}$ will denote the disjoint union of the two links in $S^{3}$. Then using the above examples we see that if $L^{\prime}$ is the link in $5,6,8$ or 9 above then

$$
\begin{aligned}
& B_{L+L^{\prime}}=B_{L} \sharp S^{2} \times D^{2} \text { if } L^{\prime}=\bigcirc_{0} \\
& B_{L+L^{\prime}}=B_{L} \# \pm C P^{2} \text { if } L^{\prime}= \pm 1 \\
& B_{L+L^{\prime}}=B_{L} \# P \# Q \text { if } L^{\prime}= \\
& B_{L+L^{\prime}}=B_{L} \# S^{2} \times S^{2} \text { if } L^{\prime}=\bigodot_{0} 0
\end{aligned}
$$

where $q$ denotes the boundary connected sum and \# is the connected sum in the interior of the manifold with boundary. Notice that $\chi_{L^{\prime}}\left(\chi_{L}(M)\right)$ is $\chi_{L}(M) \# S^{2} \times S^{1}$, in case (10) and $\chi_{L}\left(\chi_{L}(M)\right)=\chi_{L}(M)$ in cases (11), (12), (13). 
There are two basic 'moves' introduced by Kirby in manipulating such framed link pictures. We assume henceforth that our 4-manifold $B$ is simply $B^{4}$.

Move 1. $L \rightarrow L+\bigcirc_{+1}$. This move keeps $\chi_{L}(M)$ fixed but changes $B_{L}$ to $B_{L} \# \pm \mathbf{C} P^{2}$ or in reverse changes $B_{L} \# \pm \mathbf{C} P^{2}$ to $B_{L}$.

Move 2. Given two circles $\gamma_{i}$ and $\gamma_{j}$ in $L$ we "add" $\gamma_{i}$ to $\gamma_{j}$ as follows. Using the framing $n_{i}$ of $\gamma_{i}$, let $\tilde{\gamma}_{i}$ be an $n_{i}$-parallel curve of $\gamma_{i}$. Now change $L$ by replacing $\gamma_{j}$ with $\gamma_{j}^{\prime}=\tilde{\gamma}_{i}{ }_{b} \gamma_{j}$, where $b$ is a band connecting $\tilde{\gamma}_{i}$ and $\gamma_{j}$ and missing the rest of $L$.

This move corresponds in $B_{L}$ to adding (subtracting) the $j$ th handle to (from) the $i$ th handle. The new framing $n_{j}^{\prime}$ equals $\gamma_{j}+\gamma_{i} \pm 2 a_{i j}$ where $a_{i j}$ is the linking number of $\gamma_{i}$ and $\gamma_{j}$ (after they have been assigned orientations). (The linking number $l k(\xi, \eta)$ of disjoint knots $\xi$ and $\eta$ in $S^{3}$ can be defined as the image of the homology class $[\xi]$ in $H_{1}\left(S^{3}-\eta\right) \approx \mathrm{Z}$, where $S^{3}, \xi, \eta$ have been given fixed orientations.) The sign in the equations depends on whether or not the band $b$ preserves orientation. Notice that Move 2 changes neither $B_{L}$ nor $\chi_{L}(M)$. It simply provides a new handle decomposition for them.

EXAMPLE 3.

(i)

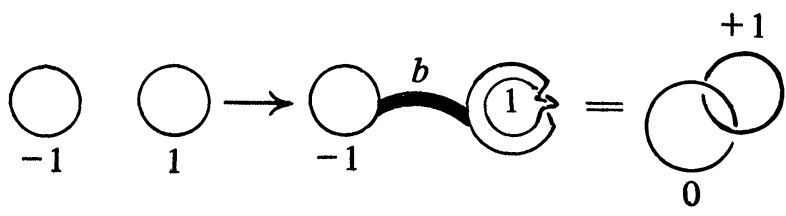

This shows that

$$
\bigcirc_{-1} \bigcirc_{1}=P \# Q-B^{4}=\bigodot_{0}
$$

(ii)

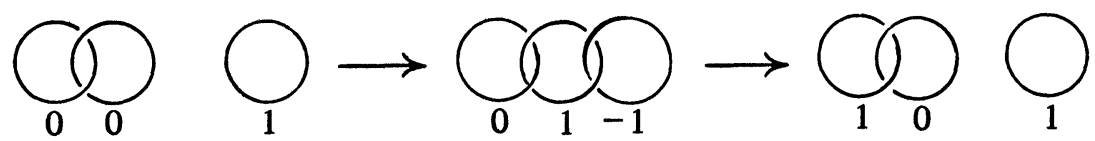

This shows that

$$
\begin{aligned}
\bigodot_{0} \bigcirc_{1} & =S^{2} \times S^{2} \# \mathbf{C} P^{2}-B^{4} \\
& =\bigodot_{1} \bigcap_{1}=P \# Q \# \mathbf{C} P^{2}-B^{4}=2 P \# Q-B^{4}
\end{aligned}
$$

Thus one can replace Move 2 by the following generalized Move $2^{\prime}$ which it implies.

Move $2^{\prime}$. Let $L$ be a link containing the portion $(L)$ pictured below. Then go from $L$ to $L^{\prime}$ or back where $L^{\prime}$ is the link identical to $L$ except that the portion $(L)$ of $L$ has been changed to $\left(L^{\prime}\right)$. 


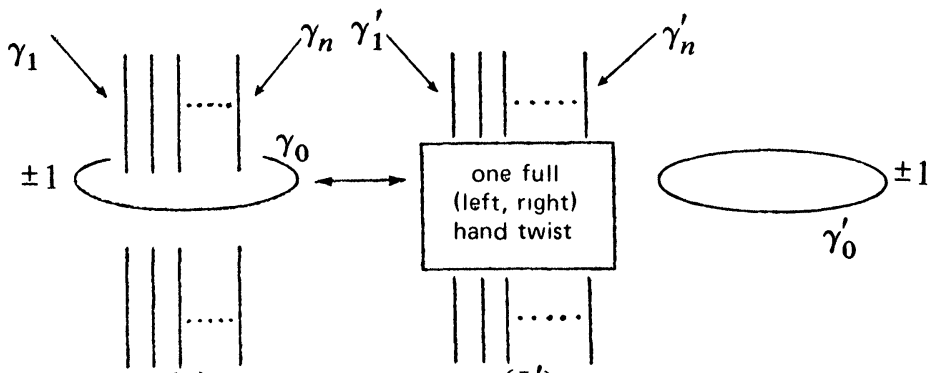

(L)

$\left(L^{\prime}\right)$

The linking matrix $\lambda_{L^{\prime}}$ for $L^{\prime}$ is then given by

$$
\begin{aligned}
& l k\left(\gamma_{i}^{\prime}, \gamma_{j}^{\prime}\right) \quad \text { if } i \neq j \\
\left(\lambda_{L}\right)_{i i}= & \left(\lambda_{L}\right)_{i i} \mp\left[\left(\lambda_{L}\right)_{0 i}\right]^{2} \quad \text { if } i \neq 0 \\
= & \pm 1 \quad \text { if } i=j=0,
\end{aligned}
$$

where $\lambda_{L}$ is the linking matrix for $L$.

In case $n=1$ or $n=2$ the equivalences are pictured below.

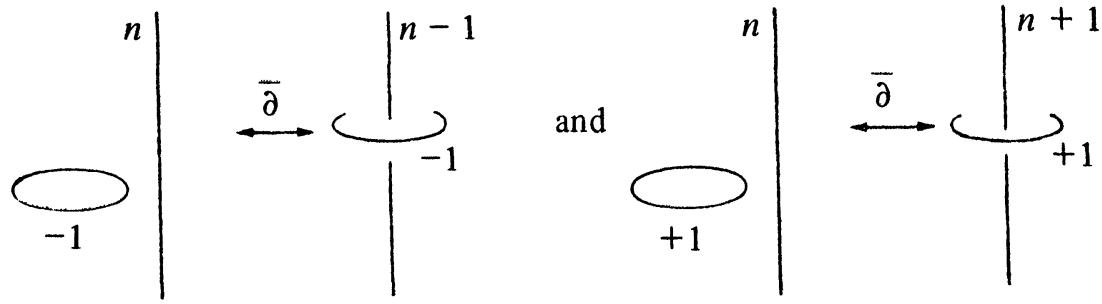

1 -strand
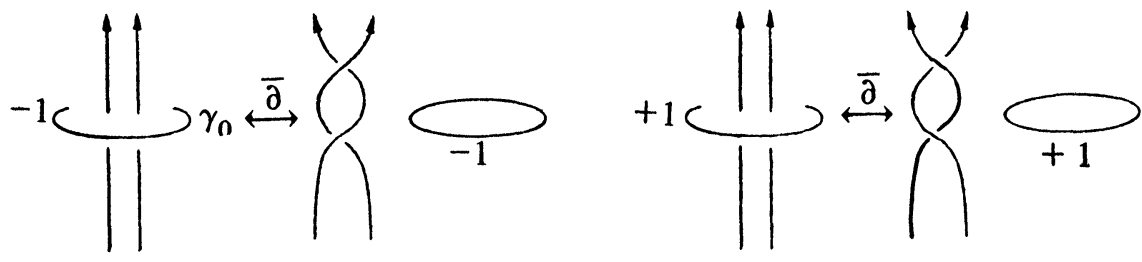

2-strands

Move $2^{\prime}$ proves very convenient when actually computing link equivalences.

Framed links as we have defined them only represent manifolds having a handle decomposition containing a 0 -handle and some 2-handles. We thus define a generalized framed link by first adding in pairs of 3-balls representing the 1-handles. Note that if 1-handles are allowed we must broaden our definition of an embedded circle to include the following type of example

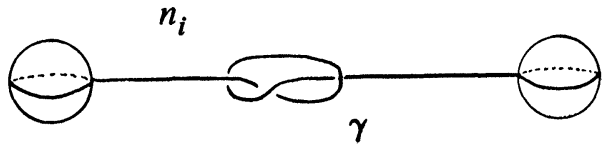

In this picture the curve $\gamma$ with framing $n_{i}$ really represents a circle embedded in the manifold $\partial(\theta \quad \theta)=\partial\left(D^{4}+1\right.$-handle $)=S^{1} \times S^{2}$ and 
homologous to $S^{1} \times 0$ ! (We shall discuss a method of representing such an example by pairs of circles in $S^{3}$ later in this chapter

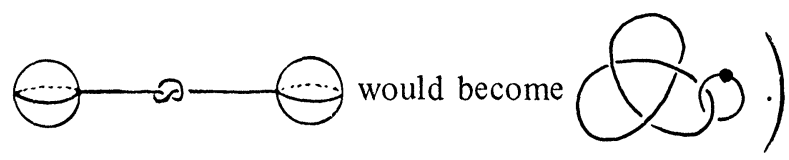

We thus redefine framed links to include pairs of embedded 3-balls representing 1-handles. To represent 3-handles we would have to draw in 2 -spheres in our pictures. However as a consequence of a result of Montesinos and Laudenbach-Poeneru [Mont 3], [LP] this is not necessary!

In fact we have the following theorem.

THEOREM 3.2 [Mont 3]. Let $M$ be a closed orientable (PL) 4-manifold with handle presentation $M=H^{0} \cup \lambda H^{1} \cup \mu H^{2} \cup \gamma H^{3} \cup H^{4}$. Then $M$ is completely determined by $H^{0} \cup \lambda H^{1} \cup \mu H^{2}$.

Thus the way the 3- and 4-handles are pasted in cannot affect the topology of $M$. We must therefore only keep track of the number, $\gamma$, of 3-handles we must add to a given link picture without worrying at all about their locations. Thus our present definition of a framed link allows us to take care of all possible handlebody decomposition of compact manifolds without boundary.

Given two framed links $L_{1}$ and $L_{2}$ we shall say $L_{1} \sim_{\partial} L_{2}$ (i.e., $L_{1}$ is boundary equivalent to $L_{2}$ ) if and only if we can go from $L_{1}$ to $L_{2}$ by a sequence of moves of types 1 and 2 . We then have:

TheORem 3.3 (KIRBy) [Kirb 1] (See also [Crg]). $L_{1} \sim_{\partial} L_{2}$ if and only if $\chi_{L_{1}}\left(S^{3}\right)$ is diffeomorphic to $\chi_{L_{2}}\left(S^{3}\right)$ (preserving orientations).

If we are interested in the 4-manifolds $B_{L}$ instead of their boundaries we must replace Move 1 by a move preserving $B_{L}$ (instead of just its boundary).

The only geometric operation we have not covered is handle cancellation and introduction.

Thus Move $1^{\prime}$ will be the introduction of a cancelling pair of 1- and 2-handles or 2- and 3-handles.

Now if $O \bigcirc$ is a 1 -handle then $\bigcirc-\infty$ will be a cancelling pair consisting of a 1-handle and a 2-handle. [Note again that the horizontal line in (1)-(1) really represents a circle in $\partial\left(B^{4} \cup\right.$ ) since the two 3-balls are identified via the 1-handle attaching them.]

An alternate way of introducing such a cancelling pair is to note that if $Q$ represents a 2-handle attached to some simply-connected manifold $V$ giving $V^{\prime}=V \nvdash S^{2} \times D^{2}$ then surgering the 2-sphere $S^{2}$ above corresponds to attaching a 1-handle to $V$ giving $\chi\left(V^{\prime}\right)=V \nvdash S^{1} \times D^{3}$.

We can then thus represent a 1-handle as a surgered 2-handle which we write 
Notice that
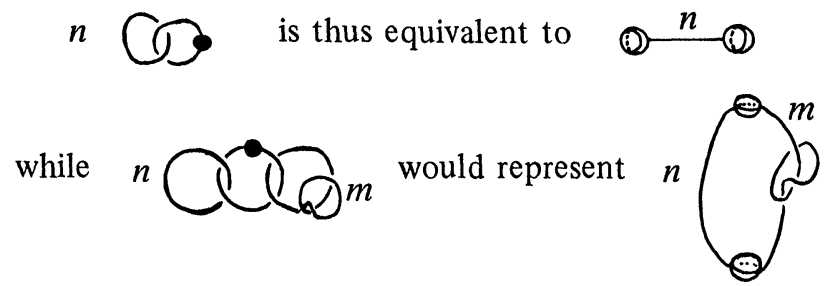

(i.e. going through $\bigcirc$ is the same as going 'over' the 1-handle). To introduce a cancelling 2-3 pair we put down $\bigcirc$ and keep track of the complementary 3-handle without drawing it in.

Move $1^{\prime}$ is then:

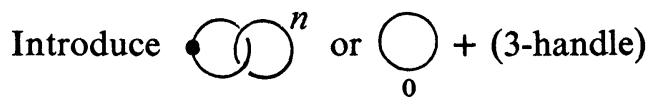

We say $L_{1} \sim L_{2}$ if we can go between them by moves of type $1^{\prime}$ and type 2 . As an analogue of Theorem 3.3 we then have Theorem 3.3' (Sá) [Kirb 1, Sá] $L_{1} \sim L_{2}$ if and only if $B_{L_{1}}$ is diffeomorphic to $B_{L_{2}}$. (See Sá [Sá] for a complete proof.)

Notice that if $V_{1}$ and $V_{2}$ are simply-connected 4-manifolds we can always write down link-pictures for $V_{1}^{\prime}=V_{1}-\{4$-ball $\}$ and $V_{2}^{\prime}=V_{2}-\{4$-ball $\}$. Clearly $V_{1}=V_{2}$ if and only if $V_{1}^{\prime}=V_{2}^{\prime}$ and thus if $V_{1}^{\prime}=B_{L_{1}}$ and $V_{2}^{\prime}=B_{L_{2}}$ then we can show that $V_{1}$ is diffeomorphic to $V_{2}$ by showing that $L_{1} \sim L_{2}$.

We apply these techniques in a few cases. (More applications will be found in later chapters.)

3.2 Handlebody decompositions of 4-manifolds. The simplest application of the link calculus arises in Example 3(2) above where

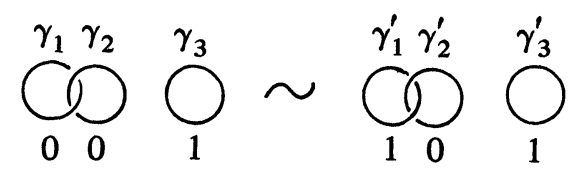

shows that $S^{2} \times S^{2} \# P=2 P \# Q$.

We note that in the above example the matrix $\left(a_{i j}\right)$ where $a_{i j}=l k\left(\gamma_{i}, \gamma_{j}\right)$ and $a_{i i}=n_{i}$ is, in fact, the intersection matrix for the resulting 4-manifold.

In fact this is a direct consequence of the definition of a handlebody decomposition. In general if $L$ is a framed link with components $\gamma_{i}$ with framing $n_{i}$, then setting $a_{i j}=l k\left(\gamma_{i}, \gamma_{j}\right), a_{i i}=n_{i}$ gives us a matrix $A_{L}$ representing the intersection form on $B_{L}$. We note that $H_{1}\left(\partial B_{L}\right)=0$ if and only if $A_{L}$ is unimodular. Thus to recognize a homology 3-sphere as the boundary corresponding to a link diagram we can simply construct the linking matrix $A_{L}$ described above and compute its matrix. In particular if $K$ is any knot then $(K, \pm 1)$ will be a homology sphere.

In Chapter 4 we shall discuss some of the 4-dimensional problems associated with homology 3-spheres and the 4-manifolds they bound. As 
preparation for some of the techniques used we present the following example of Kirby [Kirb 1].

Suppose $H$ is a homology 3-sphere which bounds an oriented 4-manifold $M$ of type II, with $b_{2}(M)=(\sigma(M))=16$. If we could also show that $H$ bounds another contractible 4-manifold $X$ then $V=M \cup X$ would be the long sought after closed spin manifold with $b_{2}(M)=(\sigma(M))=16$. For example, let

$$
\Sigma(a, b, c)=\left\{(x, y, z) \in \mathbf{C}^{3} \mid x^{a}+y^{b}+z^{c}=0\right\} \cap\left\{|x|^{2}+|y|^{2}+|z|^{2}=\varepsilon\right\} .
$$

Then $\Sigma(2,7,13)$ is a homology sphere which bounds an $M$ as above. In fact, using some techniques from the theory of resolutions of singularities we can see that $\Sigma(2,7,13)$ is precisely $\chi_{L}\left(S^{3}\right)$ where $L$ is the link

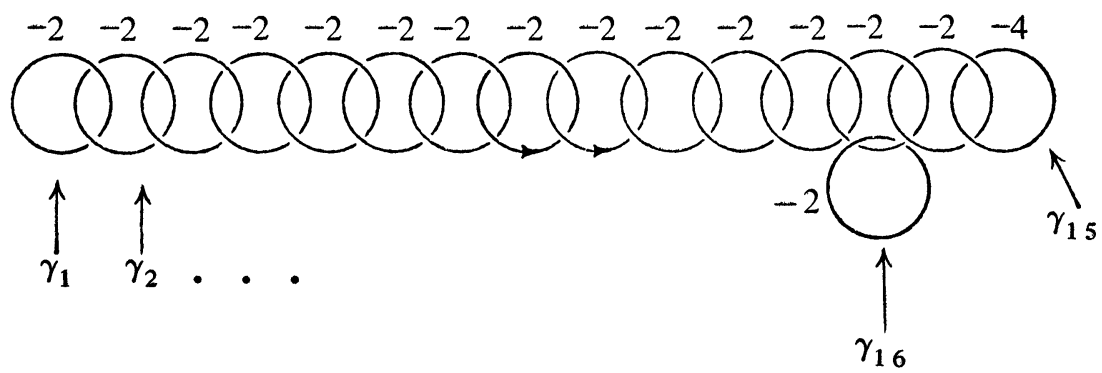

We claim that $\Sigma(2,7,13)$ is also the boundary of $\chi_{L^{\prime}}\left(S^{3}\right)$.

$$
L^{\prime}=-1 \text { (This is the }(2,7) \text { torus knot.) }
$$

To show that $\chi_{L}\left(S^{3}\right)=\chi_{L^{\prime}}\left(S^{3}\right)$ we can use moves 1 and 2 to obtain

$$
L^{\prime}+\bigcirc_{1} \rightarrow \overbrace{\gamma_{1}^{1} \gamma_{2}}^{1-1-2} \ldots \text { (rest of picture is the same). }
$$

We can use the $\bigcirc_{\gamma_{1}^{1}}^{-1}$ above to split off successive components and arrive at

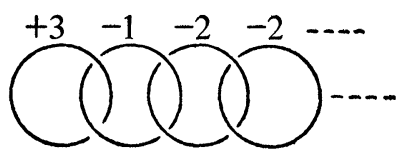

We iterate, obtaining first

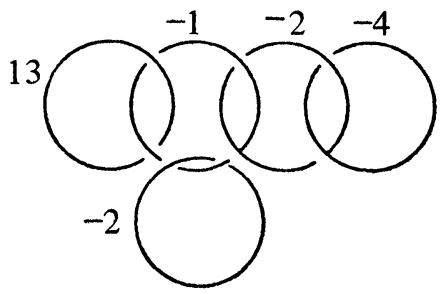


and then by adding additional $\bigcap_{-1}$ we get
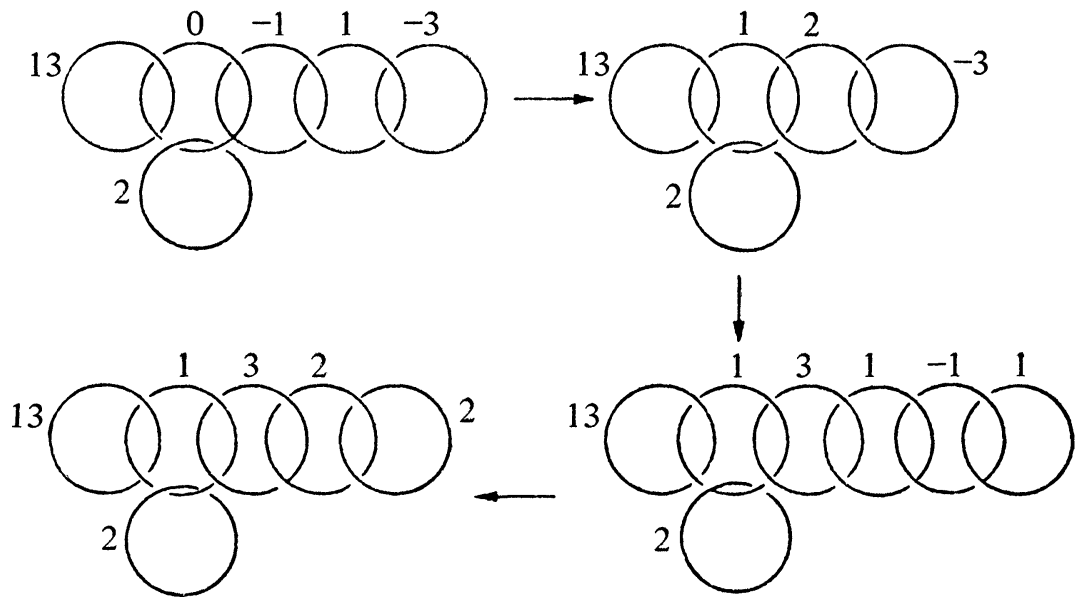

Now removing +1 circles successively we obtain as desired.


The problem then remains to show that $\Sigma(2,7,13)$ bounds a contractible manifold. One might try to solve this by constructing contractible 4-manifolds and examining their boundaries.

The first example of a compact contractible 4-manifold which is not $D^{4}$ 
was constructed by Mazur [Mz]. In our notation Mazur's manifold $W$ is

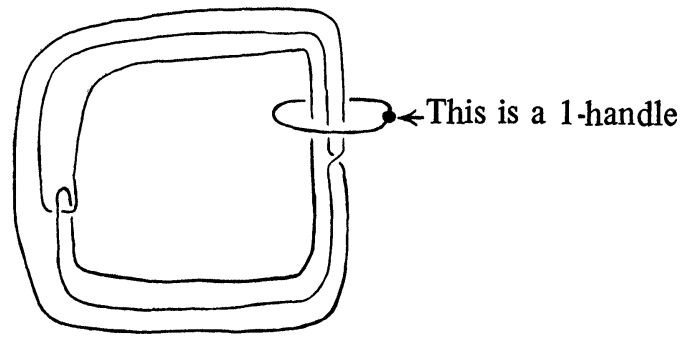

[We have attached a 2-handle to $S^{1} \times B^{3}$ along, a framed circle homologous to but not isotopic to $S^{1} \times *$ in $S^{1} \times S^{2}$.]

In [AK 2] Kirby and Akbulut defined Mazur manifolds $W^{-}(l, k)$, $W^{+}(l, k)$ as follows:
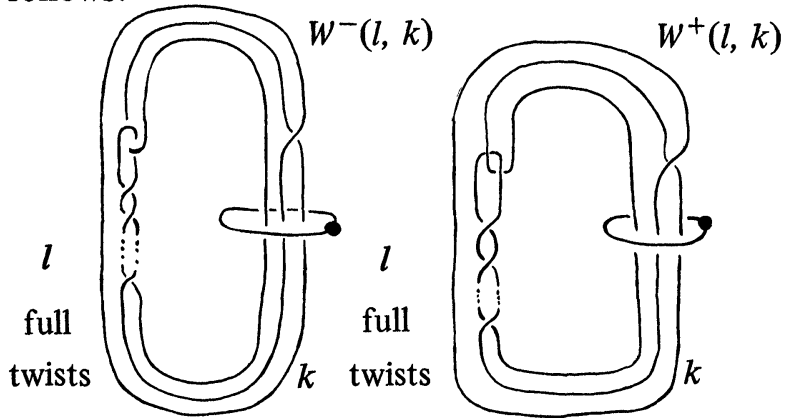

All of the manifolds can be seen to be contractible by simply noticing that $W^{-}(l, k) \times R \approx W^{+}(l, k) \times R \approx R^{5}$. [In fact, Mazur [Mz] showed that whenever one attaches a 2-handle to $S^{1} \times B^{3}$ by a curve $C$ in $\partial\left(S^{1} \times B^{3}\right)=$ $S^{1} \times S^{2}$ such that $C$ generates $H_{1}\left(S^{1} \times S^{2}\right)$ one obtains a manifold $W_{C}$ with $W_{C} \times I \approx R^{5}$. To arrange things so that $\pi_{1}\left(\partial W_{C}\right) \neq 0$ one must choose $C$ so it is not of the same knot type as the standard $S^{1} \times 1 \hookrightarrow S^{1} \times S^{2}$.]

Then using the calculus of links one calculates $\partial W^{-}(l, k)$ and $\partial W^{+}(l, k)$.

The following results are obtained:

Proposition 3.4.

(1) $\partial W^{ \pm}(l, k)=\partial W^{ \pm}(l+1, k-1)$,

(2) $\partial W^{-}(l, k) \approx \partial W^{+}(-l+2,-k+1)$,

(3) (a) $\partial W^{+}(0,0) \approx \Sigma(2,5,7)$,

(b) $\partial W^{+}(-1,0) \approx \Sigma(3,4,5)$

(c) $\partial W^{+}(1,0) \approx \Sigma(2,3,13)$.

To further demonstrate the techniques of the link calculus we include the proof of (3)(a) (taken from [AK 2]).

We have

by definition.

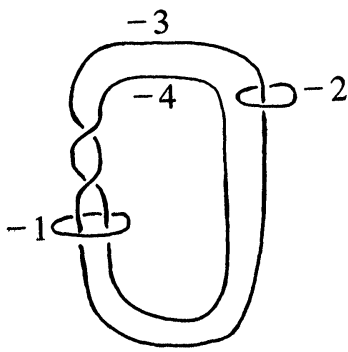


Now
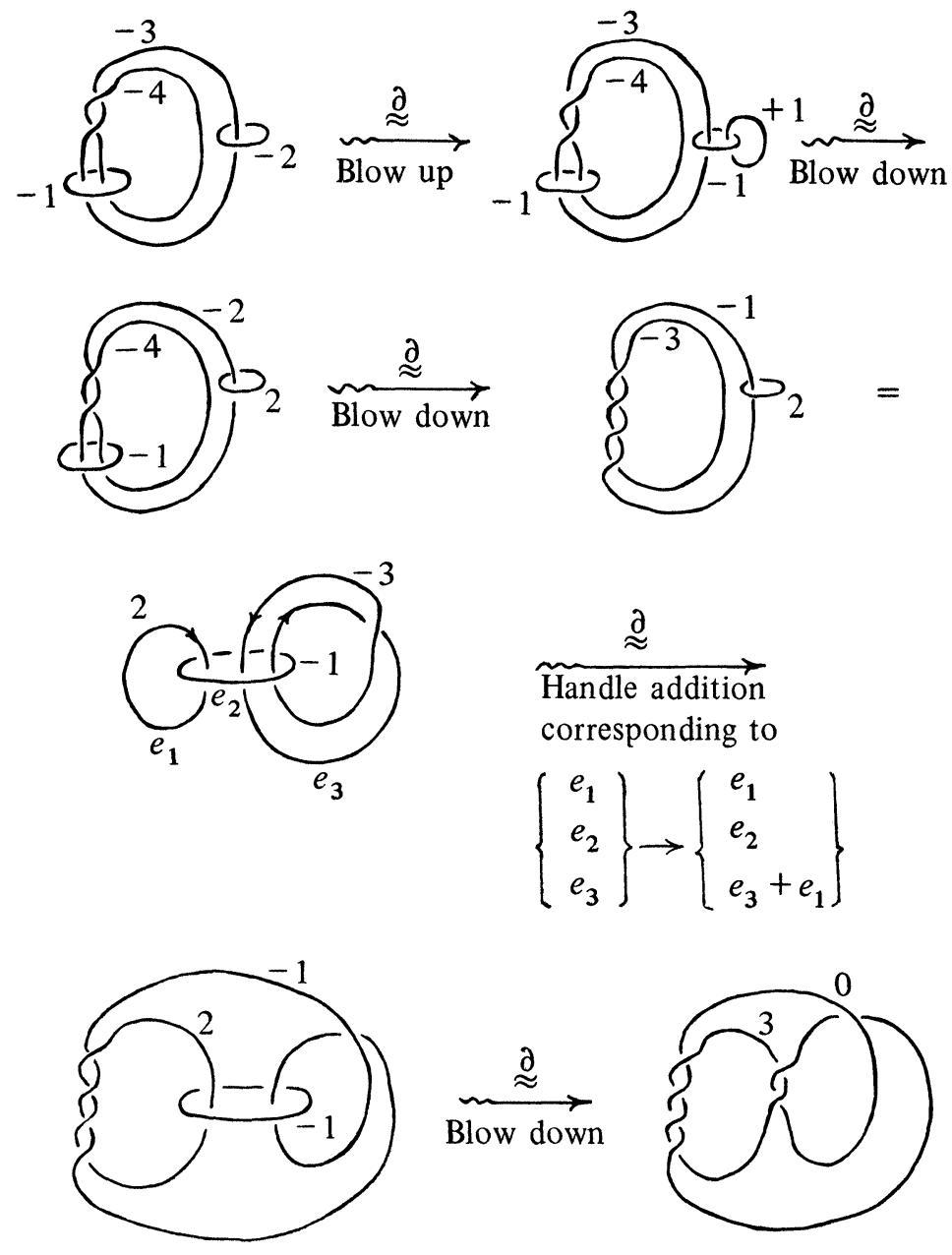

$$
=
$$
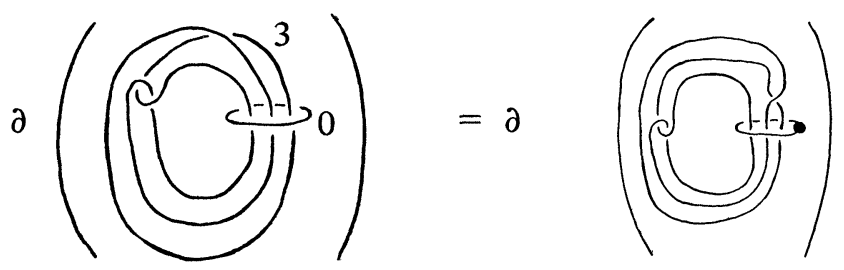

(where we have changed the 2-handle $O \circ$ to the 1-handle $Q$ in the interior of the manifold)

$=\partial W^{-}(0,3) \approx \partial W^{-}(1,2) \approx \partial W^{-}(2,1)$. From (1) of Proposition 3.4 $=\partial W^{+}(0,0)$ from (2) of Proposition 3.4. 
In [Ram], Ramanujam has constructed a nonsingular complex affine algebraic surface $V^{2}$ which is rational and contractible ( $\partial V$ is a homology but not a homotopy 3-sphere) such that $V^{2}$ is not analytically equivalent to $\mathbf{C}^{2}$ ! Kirby [Kirb 5] has found a framed link. $L$ representing $V$ and shown $V$ to be essentially a 'Mazur' manifold. $V \times \mathbf{R}^{2}$ is homeomorphic to $\mathbf{R}^{6}$ but it is not known whether $V \times \mathbf{C}$ is analytically isomorphic to $\mathbf{C}^{3}$. The construction of $V$ is as follows.

Let $C_{1}$ be a cubic curve in $\mathbf{C} P^{2}$ with a cusp. Let $C_{2}$ be a nondegenerate conic meeting $C_{1}$ at two distinct points $P, Q$ of orders 5 and 1, respectively, such that $P, Q$ are neither the cusp nor inflection point of $C_{2}$. (For example take $C_{1}: x^{3}-y^{2}=0$ and $C_{2}: x^{2}-\frac{8}{15} x y+\frac{1}{9} y^{2}+\frac{1}{3} x-\frac{8}{9} y-\frac{1}{45}$ which intersect with multiplicity 5 at $(1,1)$ and multiplicity 1 at some other point $Q$.) Blow $\mathbf{C} P^{2}$ up at $Q$ to get the variety $F$ and let $C_{1}^{\prime}, C_{2}^{\prime}$ be the proper transforms of $C_{1}, C_{2}$. Then $V$ is defined as $F-C_{1}^{\prime}-C_{2}^{\prime}$. For a proof that $V$ has the requisite properties see [Ram] or [Kirb 5].

Harer, Casson and Kaplan ([HC], [Kap]) have used these methods to construct numerous other examples of homology spheres $\Sigma(a, b, c)$ which bound contractible or acyclic manifolds. As yet however no example has been found of such a homology sphere which bounds both an acyclic manifold on the one hand and a manifold with definite form of type II on the other.

3.3 Special handlebody decompositions. Another possible approach to finding a spin manifold $M$ with $b_{2}(M)=|\sigma(M)|=16$ is by directly constructing a handle decomposition of a spin manifold $W$ with $b_{2}=22$ and $|\sigma|=16$ and attempting to manipulate such a decomposition to split off $\circ$ D 0 pairs. This would then correspond to decomposing $W$ as $W=W^{\prime} \# S^{2} \times S^{2}$ with $\sigma\left(W^{\prime}\right)=\sigma(W)$ and $b_{2}\left(W^{\prime}\right)=20$.

We first exhibit a $(22,16)$ manifold constructed by Kirby and Akbulut [AK 4] which has a handle decomposition with no 1- or 3-handles. The resultant manifold is homotopy equivalent to $V_{4}$ but it is not known whether it is diffeomorphic to it.

Let

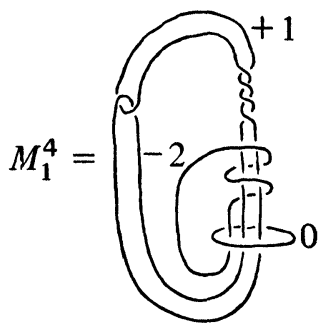

Then by the 'calculus' one shows that $\partial M_{1}^{4}=S^{3}$.

Now let

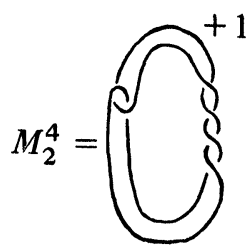

We note that $M_{1}^{4}=M_{2}^{4} \cup h_{11} \cup h_{22}$ where $h_{11}, h_{22}$ are the 2-handles attached to the circles with framing 0 and -2 in the link diagram for $M_{1}^{4}$. 
By successive moves on $M_{2}^{4}$ one obtains the manifold $N_{2}^{4}$ pictured below, preserving $\partial M_{2}^{4}=\partial N_{2}^{4}$.

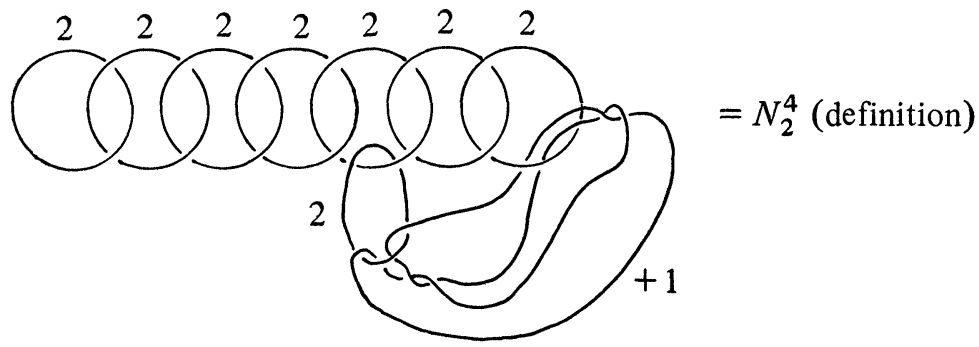

Let

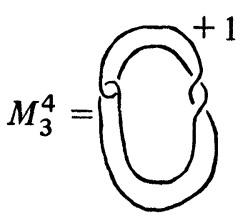

Then $N_{2}^{4}=M_{3}^{4} \cup h_{21} \cup \cdots \cup h_{28}$ where the handles $h_{2 i}$ are the handles attached to the +2 circles in the link diagram for $N_{2}^{4}$.

Now let

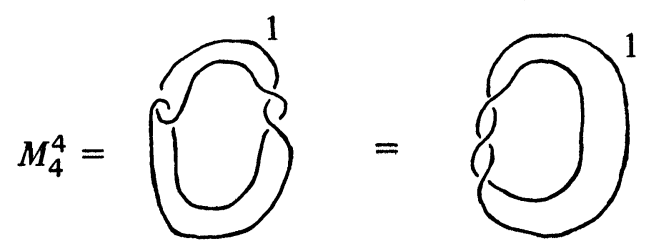

By successive moves on $M_{3}^{4}$ we get

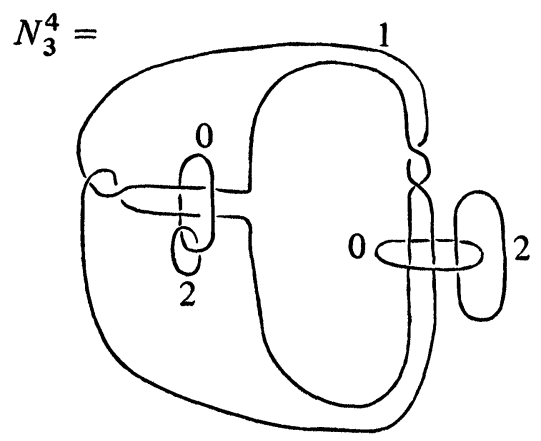

with $\partial M_{3}^{4}=\partial N_{3}^{4}$.

We again note that $N_{3}^{4}=M_{4}^{4} \cup h_{31} \cup \cdots \cup h_{34}$ where the $h_{3 i}$ are the handles attached to the circles with framings 0 and 2 in the link diagram for $\mathrm{N}_{3}^{4}$.

Lastly by successive calculus moves on $M_{4}^{4}$ we construct

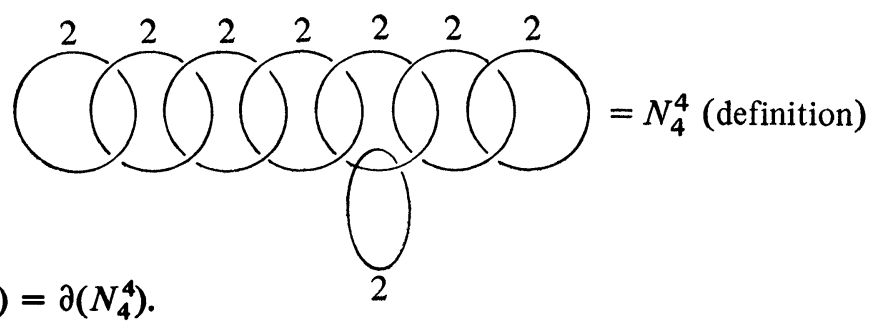

with $\partial\left(M_{4}^{4}\right)=\partial\left(N_{4}^{4}\right)$ 
We now let

$$
M^{4}=N_{4}^{4} \cup_{\partial N_{4}^{4}=\partial M_{4}^{4}}\left(\bigcup_{i=1}^{4} h_{3 i}\right) \cup_{\partial N_{3}^{4}=\partial M_{3}^{4}}\left(\bigcup_{i=1}^{8} h_{2 i}\right) \cup_{\partial N_{2}^{4}=\partial M_{2}^{4}}\left(\bigcup_{i=1}^{2} h_{1 i}\right) \text {. }
$$

Then $\partial M^{4} \approx \partial M_{1}^{4} \approx S^{3}$. Let $W=M^{4} \cup_{\partial M^{4}} B^{4}$.

Then $W$ is a simply-connected compact 4-manifold with intersection matrix determined by the linking matrix of our link diagram above. In particular we can compute $A_{L}$ to be $A_{L} \approx E_{8} \oplus E_{8} \oplus 3 U$.

Thus $W$ is a spin manifold with $b_{2}(W)=22, \sigma(W)=16$. However, although this procedure gives us an explicit picture of $W$, all attempts to split off a sublink $\circ \bigcirc D \circ$ representing a factor $U$ in the decomposition of $A_{L}$ have been unsuccessful.

Does there exist a decomposition with no 1- and 3-handles for $V_{4}$ ? In [HKK] Harer, Kas and Kirby answer this question in the affirmative by explicitly constructing such a decomposition. Their link picture is exhibited in Figure 3.2.

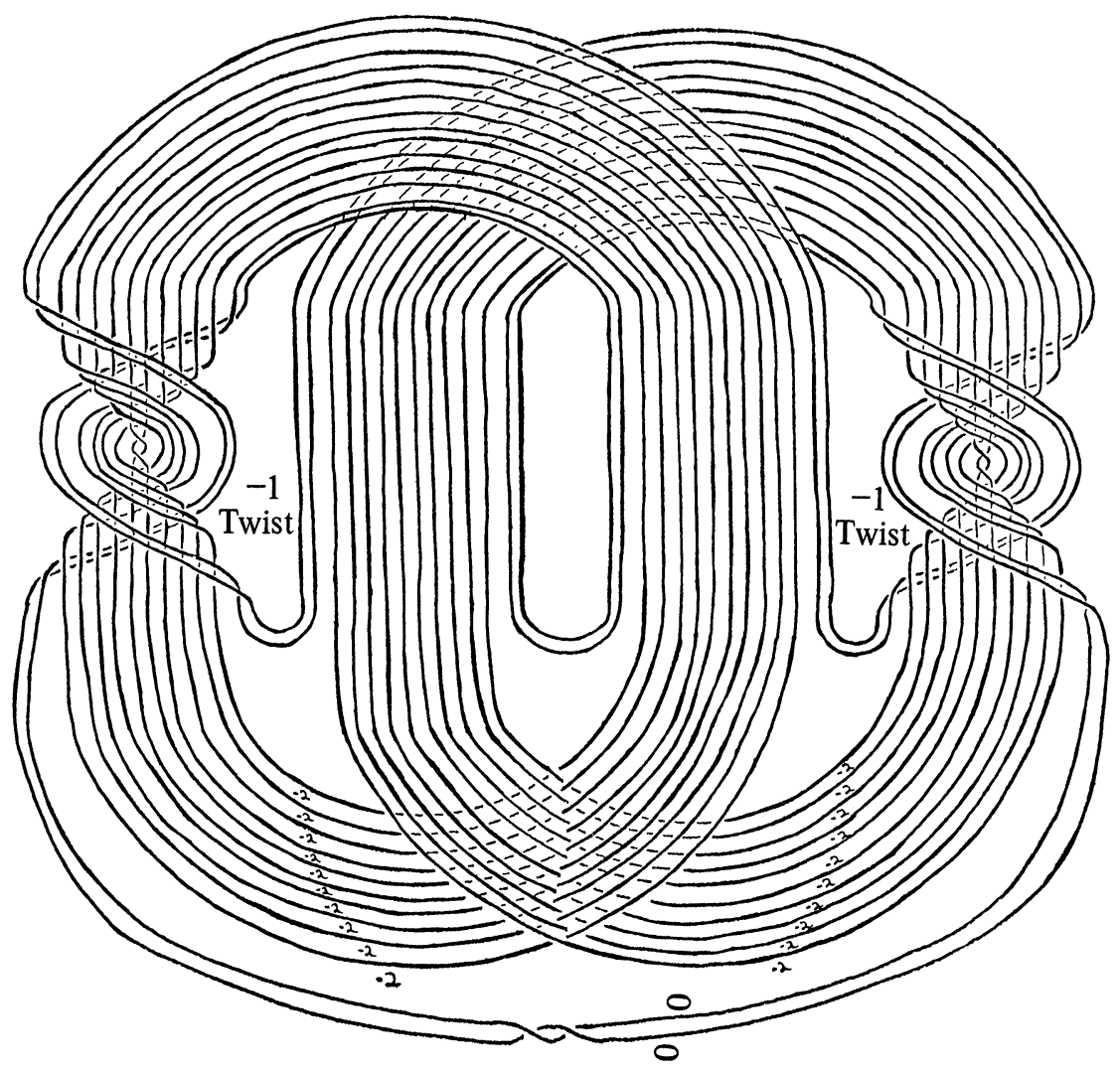

A link picture of $V_{4}$.

FIGURE 3.2 
Now let $M^{4}$ be a simply-connected 4-manifold. $M^{4}$ will be saic to admit a special handlebody decomposition if it admits a handle decomposition with one 0-handle, $b_{2}\left(M^{4}\right)$ 2-handles and one 4-handle. In this case $M^{4}$ can be completely represented by a framed link $L$ [with no 1-handles (or 3-handles) in it]. It is an open question whether every simply-connected (PL) 4-manifold admits a special handlebody decomposition. In the algebraic case Rudolph [Rd] showed that every nonsingular hypersurface $V_{n}$ of $\mathbf{C} P^{3}$ admits a decomposition with no 3-handles and [HKK] showed that $V_{4}$ admits a special decomposition. $\left(V_{1}, V_{2}, V_{3}\right.$ clearly admit such decompositions since they are rational.) In [Har], [At 3] it is shown that all $V_{n} \subset \mathbf{C} P^{3}$ admit such decomposition and in [Man 3] it is shown that all complete intersections and simply-connected elliptic surfaces (with no more than one multiple fiber) have special decompositions.

Casson [Cas 3] however has shown that there exists a compact simply-connected PL 4-manifold with $\partial M \neq 0$ requiring 1-handles in any handlebody decomposition. This result follows from the following observation.

Suppose $M$ is a simply-connected 4-manifold with $\partial M \neq 0$ having a handle decomposition $M=D^{4} \cup \cup{ }_{i=1}^{n} h_{i}^{(2)}$. Then $\overline{M-D^{4}}$ gives a cobordism between $S^{3}$ and $\partial M$ having only 2 -handles. However adding a 2 -handle to $\partial M$ has the effect of adding one new generator and one new relation to $\pi_{1}(\partial M)$. Thus the existence of a cobordism between $\partial M$ and $S^{3}$ having only 2-handles means that the group $\pi_{1}(\partial M)$ can be trivialized by adding the same number of generators and relations. Gerstenhaber and Rothaus [GR] have shown however that there exist finitely-presented groups $\{G\}$ which cannot be trivialized in this fashion, and Casson has shown that there exists a contractible 4-manifold $M$ with $\pi_{1}(\partial M) \in\{G\}$. Thus $M$ cannot have a decomposition with no 1-handles. (Note that in high dimensions such a counterexample is impossible as Wall [Wa 3] has shown that algebraic connectivity $\Rightarrow$ geometrical connectivity for manifold $M$ with $\operatorname{dim}(\partial M) \geqslant 5$.)

We note that a special handlebody decomposition and the corresponding link picture is usually quite useful in proving that a given surface is almost completely decomposable. In Figure 3.3 the first step in a direct proof, using link calculus moves, that $V_{4}$ is $A C D$ is shown.

Recently Akbulut [At 3] has developed new techniques for producing special decompositions of branched cyclic covers. Using these techniques he and Kirby [AK 3] show that direct proofs of almost complete decomposability can be demonstrated using the 'calculus'. For example they show

THEOREM 3.5. Let $V$ be a 2-fold cyclic covering of $\mathbf{C} P^{2}$ branched over a curve of degree $2 n$. Then $V$ is $A C D$.

Cyclic covers are, of course, not sufficient to generate all oriented 4-manifolds. In dimension 3 Hilden and Montesinos [Hd], [Mont 1], [Mont 4] have shown that every oriented 3-fold $M^{3}$ is a 3-fold dihedral covering manifold of $S^{3}$. In [Mont 2], Montesinos has shown that every orientable 4-manifold $V$ having a handle decomposition with no 3- or 4-handles arises as a 3-fold dihedral cover of $S^{4}$. Berstein and Edmonds [BE] have shown that $T^{4}=S^{1}$ $\times S^{1} \times S^{1} \times S^{1}$ cannot be represented as a 3 -fold covering of $S^{4}$. (More precisely they have shown that if an orientable $n$-manifold $M$ is a $p$-fold 


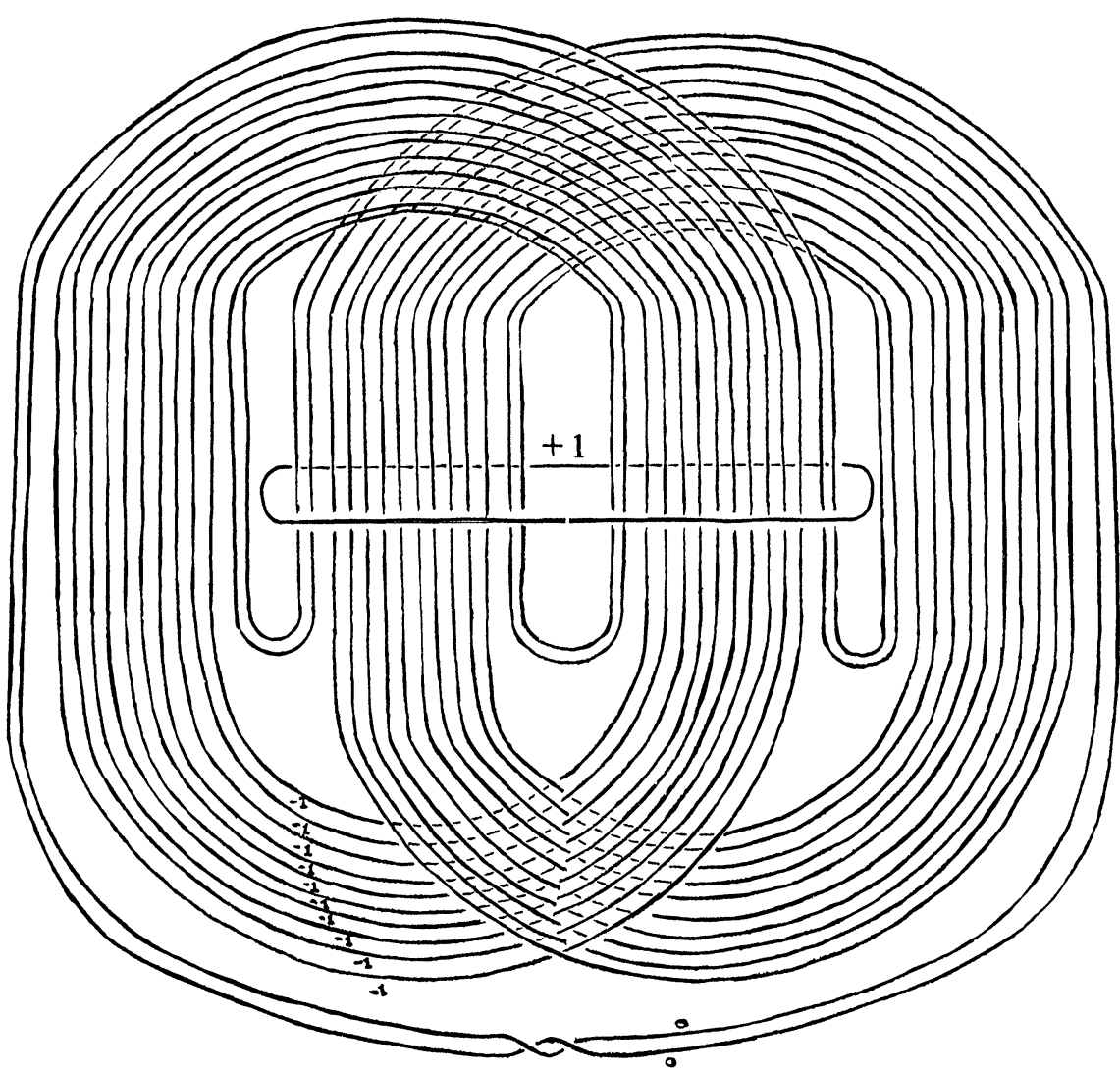

Figure 3.3 A link diagram for $V_{4} \# \mathbf{C} P^{2}$.

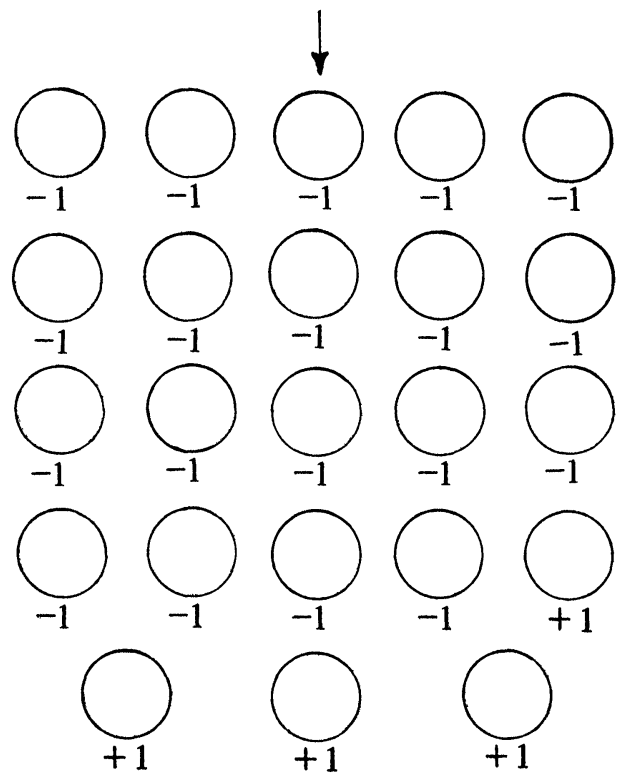

Figure 3.3a The result of type 2 moves on Figure 3.3 
covering of $S^{4}$ then $p>$ cup length $(M)$, where the cup length of a manifold $M$ is the maximum integer $r$ such that there exist $\gamma_{1}, \ldots, \gamma_{r} \in H^{*}(M, Z)$ with $\gamma_{1} \cup \cdots \cup \gamma_{r} \neq 0$. By a theorem of Alexander [Ax 1] every orientable $n$-manifold $M$ is a $p$-fold covering of $S^{4}$ for some finite $p$.)

The construction of dihedral covering manifolds for a given 4-manifold is quite a bit more complicated than the construction of cyclic covers. In [CS 4] Cappell and Shaneson have indicated a novel approach to the construction of dihedral covers $M$ of $B^{4}$ such that $\partial M$ is a dihedral covering manifold of $S^{3}$ branched over a knot and shown how the $\mu$-invariant of $\partial M$ can be calculated. (We discuss $\mu$-invariants next.) Akbulut [At 3] has noted that their method can be adapted to give link diagrams of more general dihedral covers.

In accordance with the results mentioned obtained above Montesinos and Edmonds have conjectured:

CONJECTURE. Let $M$ be a simply-connected 4-manifold. Then $M$ admits a representation as a 3 -fold branched cover of $S^{3}$.

CONJECTURE. Let $M$ be an oriented $n$-manifold. Then $M$ admits a representation as an $n$-fold branched cover of $S^{n}$.

Before closing this section we point out that it is not always possible to show that two link diagrams give equivalent manifolds without moves of type I. Thus simply showing that by adding and subtracting link components one cannot transform link $L_{1}$ to link $L_{2}$ does not suffice to conclude that $B_{L_{1}} \neq B_{L_{2}}$ or $\partial B_{L_{1}} \neq \partial B_{L_{2}}$. In fact Akbulut has shown that if $K, R$ are the framed links of Figure 3.4 then $K$ is not equivalent to $R$ (a direct computati $A$ shows that $K$ has signature -2 and $R$ has signature zero) but $B_{K}=B_{R}$ [At 1].
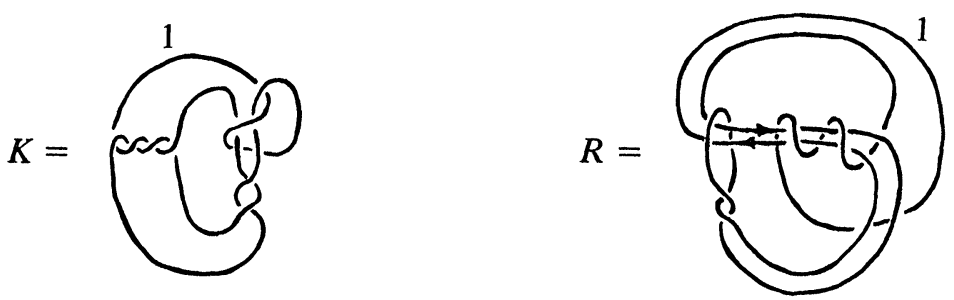

FIGURE 3.4

\section{CHAPTER 4. THE $\mu$-INVARIANTS, HOMOLOGY SPHERES AND FAKE 4-MANIFOLDS}

4.1 The $\mu$-invariant. One of the only known numerical invariants interrelating 3- and 4-dimensional topology is the $\mu$-invariant [Hirz 2], [EK] which we now discuss.

Suppose $M^{n}$ is a manifold with tangent bundle $T M$. A fixed trivialization $\mathscr{F}: T M \rightarrow M \times \mathbf{R}^{n}$ of $T M$ will be called a framing on $M$ and $(M, \mathscr{F})$ will be called a framed manifold. We recall that the stable tangent bundle $T^{s} M$ of $M$ can be geometrically realized as $T M \oplus \varepsilon^{r}$ for $r$ sufficiently large $\left(\varepsilon^{r}=M \times\right.$ $\mathbf{R}^{r}$ ) and thus a stable framing on $M$ is a fixed trivialization of some $T M \oplus \varepsilon^{r}$. Lastly, an almost framing on $T M$ is a framing on $T(M-\{\mathrm{pt}\})$. We have: 
THEOREM 4.1 (MILNOR [M 2]); ALSO [Kap]. Let $\left(M^{3}, \mathscr{F}\right)$ be a closed oriented stably framed 3-manifold. Then there exists a compact stably framed 4-manifold $\left(W^{4}, \mathcal{G}\right)$ with $\partial W=M$ so that $\mathcal{G}$ and $\mathscr{F}$ agree on $M-\{\mathrm{pt}\}$. $\left(W^{4}\right.$ can, in fact, always be found with $\pi_{1}\left(W^{4}\right)=0$.)

PROOF. We shall later outline Kaplan's proof of the above result.

A result of Whitehead [M 3, §4] shows that stably framed implies almost framed and that for connected manifolds with boundary, framed $=$ almost framed = stably framed. Thus $W$ above will in fact be framed. We also recall that a framed (stably framed) manifold is sometimes called parallelizable (stably or $S$-parallelizable or $\pi$-manifold) and an almost framed manifold is called almost parallelizable.

We note that since $W$ is framed the form $L_{W}$ must be even. Thus if $\left(W^{\prime}, \mathcal{G}^{\prime}\right)$ is any other 4-manifold satisfying the conclusion of Theorem 4.1 we can form $V=W \cup W^{\prime}$ and using our stable framings show that $V$ is also stably framed. Then using Novikov additivity [Hirz 2] we find $\sigma(W)-\sigma\left(W^{\prime}\right)$ $=\sigma(V)$ and by Rohlin's theorem $\sigma(V) \equiv 0(\bmod 16)$.

We are thus led to the following.

Definition 4.2. Let $(M, \mathscr{F})$ be a stably framed 3-manifold and suppose $(W, \mathcal{G})$ is a framed 4-manifold with $\partial W=M$ and $\mathscr{F}=\mathcal{G}$ on $M-\{\mathrm{pt}\}$.

Then set $\mu(M, \mathscr{F})=\sigma(W) \bmod 16$ (by our discussion above $\mu(M, \mathscr{F})$ is clearly well defined).

We note that to define $\mu$ it is enough to specify some almost framing on $M^{3}$. However by obstruction theory since $\pi_{2}(\mathrm{SO}(3))=0$ we find that the almost framings of $M^{3}$ will be in 1-to-1 correspondence with the elements of $H^{1}\left(M, \pi_{1}(\mathrm{SO}(3))\right)=H^{1}\left(M ; Z_{2}\right)$. Thus if $M^{3}$ is a $\mathbf{Z}_{2}$-homology sphere it has a unique almost framing. Thus for $\mathbf{Z}_{2}$-homology spheres $M$ one can speak of $\mu(M)$ without referring to an almost framing of $M$. In this case it can further be shown that $\mu(M)$ is in fact an invariant of the $h$-cobordism class of $M$.

Now let $\mathcal{H}^{3}(G)$ be the abelian group of $h$-cobodism classes of $G$-homology 3-spheres. (The group structure is given by the connected sum operation. [ $\left.S^{3}\right]$ is the identity and $\left[-M^{3}\right]$ is the inverse of $M^{3}$, where $-M$ is $M$ with orientation reversed.) Then it is not difficult to show that $\mu: \mathcal{H}^{3}(\mathbf{Z} / 2 \mathbf{Z}) \rightarrow \mathbf{Z}_{16}$ is a homomorphism. It is clear that $\operatorname{Im} \mu$ is contained in a subgroup of $\mathbf{Z}_{16}$ isomorphic to $\mathbf{Z} / 8 \mathbf{Z}$ and by calculation of $\mu$-invariants of lens spaces one can show that $\mu$ is in fact onto this subgroup. If $M$ is a $\mathbf{Z}$-homology sphere then for $W$ defined as in the theorem we have $\sigma(W) \equiv 0(\bmod 8)$. Thus in fact $\mu$ : $\mathcal{H C}^{3}(\mathbf{Z}) \rightarrow \mathbf{Z}_{8}$ is onto a subgroup $\mathbf{Z}_{2} \approx \mathbf{Z} / \mathbf{2 Z}$. (To see that $\mu: \mathcal{H C}^{3}(\mathbf{Z}) \rightarrow \mathbf{Z}_{\mathbf{2}}$ is onto we simply note that if $P$ is Poincaré's homology 3-sphere then if

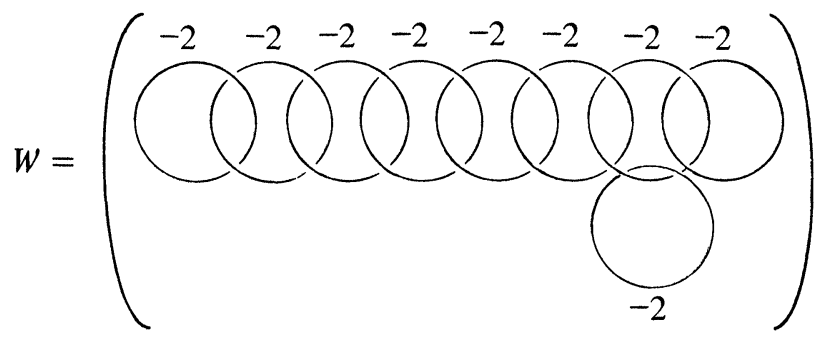


clearly $\sigma(W)=8$ and using Kirby moves one can show that

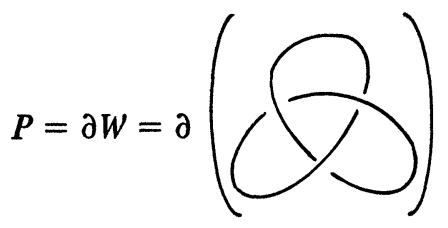

1

Thus $\mu(P)=8$ which generates the $Z_{2}$ above.

We consider some applications of the $\mu$-invariant. We see immediately that by Alexander duality if $M \in \mathfrak{H}^{3}(\mathbf{Z} / 2 \mathrm{Z})$ is embedded in $\mathbf{R}^{4}$ then $\mu(M)=0$.

Now since $\mu$ is a diffeomorphism invariant if we could find a homotopy Z-sphere $M$ with $\mu(M) \neq 0$ then $M$ would be a counterexample to the Poincaré conjecture in dimension 3. In [CS 3], Cappell and Shaneson have developed a formula for computing the $\mu$-invariant for any $p$-fold dihedral cover $M$ of $S^{3}$, branched over a knot such that $M$ is a $Z / 2 Z$ homologysphere. Since by [Mont 1], [Mont 4], [Hd] every orientable 3-manifold can be represented as such a cover it might be possible to find a counterexample to the Poincare conjecture by constructing the $p$-fold dihedral covering manifolds $M_{p}(K)$ associated with all the knots $K$ in the Conway [Con] tables, computing their fundamental groups $\pi_{1}(M)$ and $\mu$-invariants if $\pi_{1}(M)=0$. (This might profitably be done by computer.)

A possible application in dimension 4 of the $\mu$-invariant is to the problem of finding simply-connected 4-manifolds which are spin and have definite forms. In particular it would be desirable to find out when elements $M \in$ $\mathcal{F}^{3}(\mathbf{Z})$ bound contractible or acyclic 4-manifolds. Work in this direction can be found in [HC], [Kap]. Gordon has devised a construction in [Gor 1] which associates a contractible 4-manifold with boundary a Z-homology sphere to any slice knot $K \subset S^{3}$. (A knot $K \subset S^{3}$ is a slice knot if there exists a (PL) 2-disc $D^{2} \subset B^{4}$ such that $D^{4}$ intersects $\partial B^{4}=S^{3}$ transversely in $K$.) It would be of interest to determine which of Gordon's homology spheres also bound simply-connected 4-manifolds $M$ with definite even-forms, or even with even-forms for which $b_{2}(M)-|\sigma(M)|$ is as small as possible. It is thus of interest to have a more constructive proof of Theorem 4.1 above. (Milnor's proof involves the Thom construction [Thm] and does not give a wholly clear picture of the manifold $W$ constructed.) In [Kap] Kaplan proves Theorem 4.1 by constructing an explicit handlebody decomposition for $W$.

We again recall that if $L$ is a bilinear form $V \times V \rightarrow \mathbf{Z}$ on the module $V$ then $x \in V$ is characteristic for $L$ if and only if $L(x, y)+L(y, y) \equiv 0$ $(\bmod 2)$ for all $y \in V$. Now if $J$ is a framed link in $S^{3}$ with associated 4-manifold $B_{J}\left(\partial B_{J}=M_{J}\right)$ then to each sublink $R$ of $J$ there corresponds a homology class $[R]$ in $H_{2}\left(B_{J} ; Z\right) .[R]$ is representable as the union of the core of the handle over $R$ and the cone on $R$ in $B^{4}$. By a result of Thom [Thm], [R] is in fact also always representable by a smooth oriented 2-manifold. We shall call $R$ a characteristic sublink if $j_{*}[R]$ is characteristic for the homology pairing on $H_{2}\left(B_{J}, M_{J} ; Z\right)$ dual to $L_{B_{J}}\left(j_{*}: H_{2}\left(B_{J}\right) \rightarrow H_{2}\left(B_{J}, M_{J}\right)\right)$, or equivalently if any smooth oriented manifold $F$ representing $[R]$ is characteristic in 
the sense of Chapter 1. (Alternatively we define the linking matrix $\lambda$ for the link $J$ by

$$
\lambda_{i j}=\left\{\begin{array}{ll}
l k\left(K_{i}, K_{j}\right) & \text { if } i \neq j, \\
\text { framing } K_{i} & \text { if } i=j,
\end{array} \quad 1<i, j \leqslant n,\right.
$$

where $K_{1}, \ldots, K_{n}$ are the components of $J$ and $l k\left(K_{i}, K_{j}\right)$ is the algebraic linking number of the knots $K_{i}, K_{j}$. Note that $\lambda$ will always be a representative matrix for the cup-product form $L_{B_{j}}$. We have that $R$ is a characteristic sublink if and only if it is characteristic for the bilinear pairing induced by $\lambda$ on $\oplus \mathbf{Z} K_{i}$.)

We note that if a 4-manifold $W$ has characteristic submanifold $F$ then a procedure whereby $W$ could be modified so as to 'kill' $F$ would produce a new 4-manifold $W^{\prime}$ which would necessarily have $w_{2}\left(W^{\prime}\right)=0$ and thus be spin or equivalently (in this dimension) almost parallelizable. This idea is the core of Kaplan's construction. That is, given $\left(M^{3}, \mathscr{F}\right)$ one can always find a framed link representation [Lick] for it and thus one knows $M^{3}=\partial\left(B_{J}\right)$ for some framed link $J$. One then identifies a characteristic sublink $R$ of $J$ and shows how one can kill $R$ using Kirby moves. This produces $B_{J}$, with $\partial\left(B_{J^{\prime}}\right)=M$ and $w_{2}\left(B_{J^{\prime}}\right)=0$ as desired.

More concretely if $M=\partial\left(B_{J}\right)$ then corresponding to each 2-handle of $B_{J}$ there is a dual circle (the $b$-circle in the terminology of Chapter 1 ) in $M$, the attaching circle of the dual handle. The dual circles generate $H_{1}\left(M ; Z_{2}\right)$ and so the almost framing for $M$ is determined by the framing induced on the tubular neighborhoods of the dual circles. Now the framed link $J$ also induces a framing on the neighborhoods of the dual circles and the difference between the two framings gives a map $H_{1}\left(M ; Z_{2}\right) \stackrel{T}{\rightarrow} \mathbf{Z}_{2}$, i.e. an element of $H^{1}\left(M ; Z_{2}\right)$. Then finding a characteristic sublink can be reduced to finding a sublink $R$ of $J$ such that $T\left(K_{i}\right)=1 \in \mathrm{Z}_{2}$ for all the components $K_{i}$ of $R$.

In Figures 4.1-4.3 [Kap] we give examples of characteristic sublinks of given links. Killing these characteristic sublinks by Kirby moves then gives the desired manifold $B_{J^{\prime}}$. We note that the parallelizable manifold $B_{J^{\prime}}$ in Figure 4.3 produced by killing the characteristic sublink has $b_{2}\left(B_{J^{\prime}}\right)=22$, $\sigma\left(B_{J^{\prime}}\right)=16$. Furthermore $\partial\left(B_{J^{\prime}}\right)=S^{3}$ so $W=B_{J^{\prime}} \cup D^{4}$ gives another example of an almost framed 4-manifold with $b_{2}=22$ and $\sigma=16$ having a handle decomposition with no 1 - or 3-handles. $W$ is homotopy equivalent to $-V_{4}$ but it is not known if it is diffeomorphic to it.

(It is actually not strictly necessary to follow this procedure if one is only interested in computing the $\mu$-invariant of $\left(M^{3}, \mathscr{F}\right)$. The following formula of Cappell and Shaneson [CS 2]:

$$
\mu\left(M^{3}, \mathscr{F}\right) \equiv \sigma\left(L_{B_{J}}\right)+R \circ R+8 \operatorname{Arf}(J)(\bmod 16),
$$

where $M^{3}=\partial B_{J}, R$ is the characteristic sublink of $J$ and $R \circ R$ is the self-linking of $R$ given by $L_{B_{f}}(R, R)$ gives $\mu$ directly.)

Our description above of the relationship between characteristic sublinks and almost framings shows that each almost framing of $M=\partial\left(B_{J}\right)$ determines a unique characteristic sublink of $J$. Consider for example, the 
3-torus $T^{3}$ with framed link picture representation $L$ as in Figure 4.4 (i.e., $\left.\partial B_{L}=T^{3}\right) . T^{3}$ has eight distinct almost framings and the corresponding characteristic sublinks are simply the eight distinct $\mathbf{Z}_{2}$-linear combinations of the components of $L$. It can be shown [Kap] that any proper characteristic sublink $L_{i}$ of $L$ induces an almost framing $\mathscr{F}_{i}$ with $\mu\left(T^{3}, \mathscr{F}_{i}\right)=0$. However if $L$ is itself characteristic then the almost framing $\mathscr{F}_{0}$ induced by $L$ satisfies $\mu\left(T^{3}, \mathscr{F}_{0}\right)=8$. The above behavior of $T^{3}$ is, in fact, typical of a large class of 3-manifolds, and Kaplan has shown

THEOREM 4.3 [Kap]. Suppose $M$ is a closed, connected oriented 3-manifold which bounds a framed manifold of index $\sigma$. Suppose further that there exist elements $X_{i} \in H^{1}\left(M ; Z_{2}\right), i=1,2,3$, with $X_{1} \cup X_{2} \cup X_{3} \neq 0$. Then $M$ also bounds a framed manifold of index $\sigma+8$.
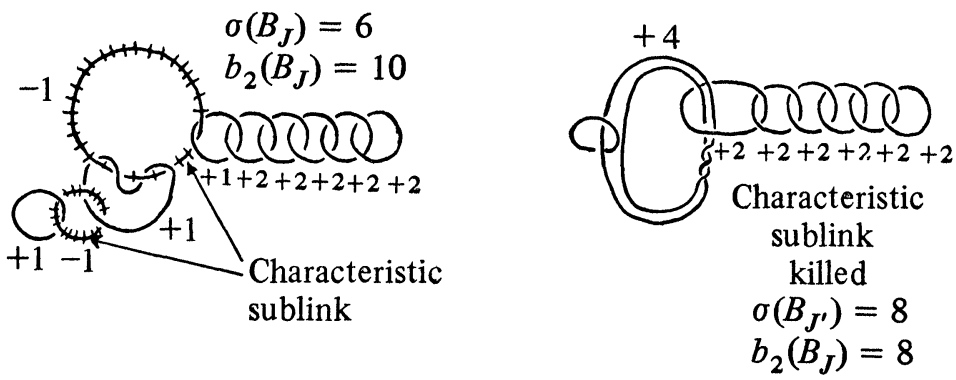

$\partial B_{J}=\partial B_{J^{\prime}}=P$

FIGURE 4.1

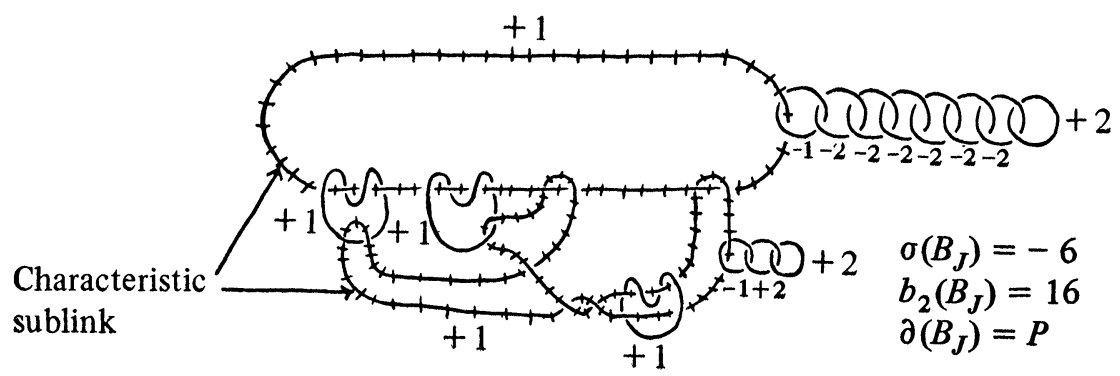

$J^{\prime}$ can be obtained by 'blowing down' the characteristic sublink indicated in the picture. The manifold $B_{J^{\prime}}$ obtained has $\sigma\left(B_{J^{\prime}}\right)=-8 ; b_{2}\left(B_{J^{\prime}}\right)=14$ and $\partial\left(B_{J^{\prime}}\right)=P$.

Gluing together the $B_{J^{\prime}}$ of Figures 1 and 2 gives a closed spin manifold $K$ with $\sigma(K)=16$ and $b_{2}(K)=22$. 


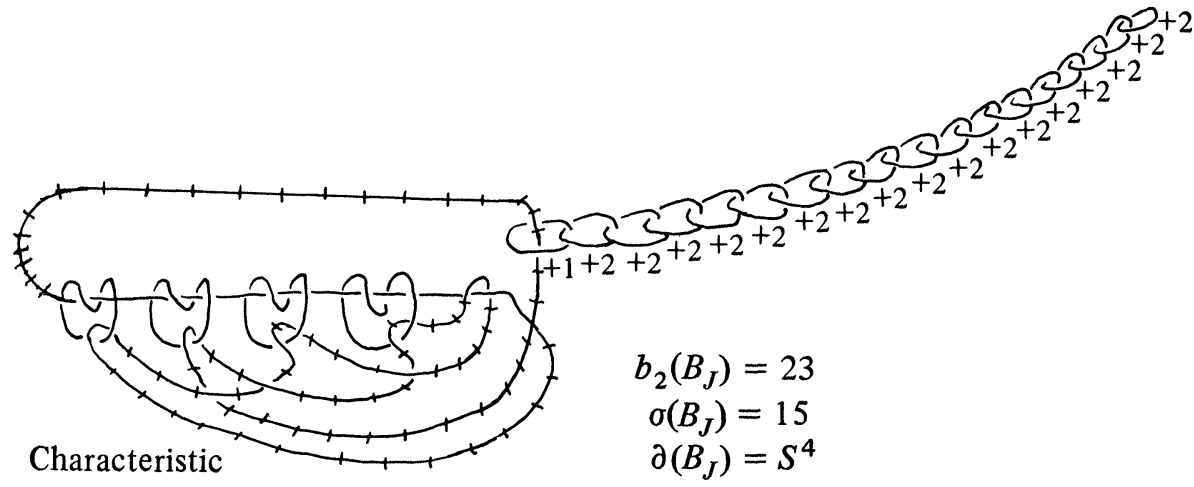

sublink

Blowing down the characteristic sublink in this picture produces a manifold $V$ with $b_{2}(V)=22 \sigma(V)=16$ with $\partial V=S^{3}$ and $L_{V}$ of type II

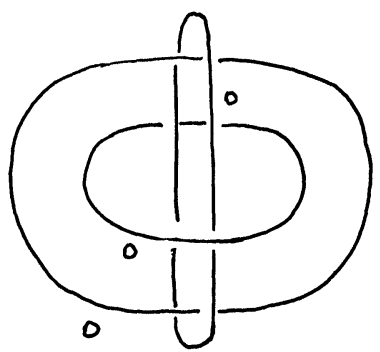

A framed link $L$ with $\partial\left(B_{L}\right)=T^{3}$

FIGURE 4.4

(Another way to establish the existence of an (almost) framing $\mathscr{F}_{0}$ on $T^{3}$ with $\mu\left(T^{3}, \mathscr{F}_{0}\right)=8$ (see also [CS 2]) is to consider the rational elliptic surface $W_{0}$ we discussed in Chapter 2. $W_{0} \rightarrow S^{2}$ is an elliptic fiber space with $W_{0}=P \# 9 Q$. We can assume without loss of generality that the fiber of $W_{0}$ over $0 \in S^{2}$ is nonsingular, and we let $T_{0}=\pi^{-1}\left(D_{0}\right)$ where $D_{0}$ is a disc about $0 \in S^{2}$ containing no critical values. Then $T_{0} \approx T^{2} \times D_{0}$ and setting $W_{0}^{*}=\overline{W_{0}-T_{0}}$ we have by Novikov additivity that $\sigma\left(W_{0}^{*}\right)=\sigma\left(W_{0}\right)=-8$. $W_{0}^{*}$ is in fact easily seen to be parallelizable (we have killed the second Steifel-Whitney class of $W_{0}$ by removing $T_{0}$ ) and thus its framings restricts to a framing $\mathcal{F}$ on $\partial W_{0}^{*}=\partial\left(T^{2} \times D_{0}\right)=T^{3}$. Then clearly $\mu\left(T^{3}, \mathscr{F}\right)=8 \bmod 16$ and $\mathscr{F}$ thus must coincide with $\mathscr{F}_{0}$ as above. If we let $f$ be the stable framing on $S^{1}$ indicated in Figure 4.5 then one can check that $\mathscr{F}_{0}=f \times f \times f$ as a framing on $T^{3}=S^{1} \times S^{1} \times S^{1}$.) 


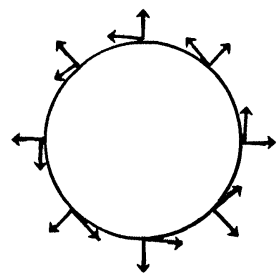

The stable framing $f$ on $S^{1}$ exhibited here is the unique framing not extendable to a (stable) framing of $D^{2}$

FIGURE 4.5

4.2 Fake 4-manifolds. An extremely beautiful use of the existence of this 'exotic' framing on $T^{3}$ has been made by Cappell and Shaneson in their construction of a compact 4-manifold $Q^{4}$, simple homotopy equivalent to $\mathbf{R} P^{4}$ but not diffeomorphic to it.

We shall indicate the geometric construction of $Q^{4}$ but suppress most of the algebra involved in proving it is not diffeomorphic to $\mathbf{R} \boldsymbol{P}^{\mathbf{4}}$. More precisely we have

THEOREM 4.4 (CAPPEll AND SHANESON) [CS 2]. Let $(X, \partial X)$ be a compact smooth connected 4-manifold with (possibly empty) boundary. Suppose $\pi_{1}(X)$ has an orientation reversing element of order 2 . Then there is a manifold $\left(Q^{4}, \partial Q\right)$ and a simple homotopy equivalence $f:(Q, \partial Q) \rightarrow(X, \partial X)$ with $f \mid \partial Q:$ $\partial Q \rightarrow \partial X$ a diffeomorphism such that $f$ is not homotopic to a diffeomorphism or PL homeomorphism.

Corollary 4.5. Let $X=\mathbf{R} P^{4}$. Then there exists a 4-manifold $Q$, simple homotopy equivalent but not PL homeomorphic or even PL s-cobordant to $X$. Furthermore for any $k \geqslant 0, Q \# k\left(S^{2} \times S^{2}\right)$ is not PL s-cobordant to $X \# k\left(S^{2} \times S^{2}\right)$. However if $V$ is any manifold with $\operatorname{dim} V \geqslant 1((\operatorname{dim} V \geqslant 2)$ if $\partial V \neq \varnothing)$ then $Q \times V$ is topologically homeomorphic to $X \times V$.

Proof. The construction of $Q^{4}$ proceeds as follows.

Let $C \subset X$ be an embedded circle in $X$ representing an order 2 orientation reversing element of $\pi_{1}(X)$.

Let $T_{C}$ be a tubular neighborhood of $C$. Then $T=T_{C}$ is the unique nontrivial nonorientable orthogonal $D^{3}$-bundle over $S^{1}$. Set $X_{0}=\overline{X-T_{C}}$ and $\partial X_{0}=H . H$ is thus a nontrivial $S^{2}$-bundle over $S^{1}$.

To construct $Q$ we construct a new manifold $M_{0}$ with $\partial M_{0}=H$ and define $Q=X_{0} \cup_{H} M_{0}$. (More accurately we construct an infinite family of manifolds $M_{0}(A)$ and set $Q(A)=X_{0} \cup_{H} M_{0}(A)$.)

Ideally we would construct $Q$ by taking $M_{0}$ to be an 'exotic' $D^{3}$-bundle over $S^{1}$ which agrees with the standard nonorientable $D^{3}$-bundle near the boundary. (Note that in dimension $n, n \geqslant 5$, exotic $D^{3}$-bundles over $T^{n-3}$ do exist! See [HS 1], [HS 2], [Sh 2].) Unfortunately we do not know if such a bundle exists. Instead we will take $M_{0}$ to be a punctured $T^{3}$-bundle over $S^{1}$ which is Z-homology equivalent to $T_{C}$.

Thus let $T^{3}=S^{1} \times S^{1} \times S^{1}$ be the 3-torus and suppose $A \in \mathrm{GL}(3, \mathrm{Z})$ with $\operatorname{det} A=-1$ and $\operatorname{det}\left(I-A^{2}\right)= \pm 1$. $A$ induces a $\operatorname{diffeomorphism} \phi_{A}$ : 
$T^{3} \rightarrow T^{3}$ with $\left(\phi_{A}\right)_{*}: \pi_{1} T^{3} \rightarrow \pi_{1} T^{3}$ equal to $A$. Clearly we can isotope $\phi_{A}$ to a diffeomorphism $\phi: T^{3} \rightarrow T^{3}$ with a fixed point $* \in T^{3}$.

[For example letting $S^{1}=\mathbf{R} / \mathbf{Z}$, define $\phi$ by $\phi\left(\theta_{1}, \theta_{2}, \theta_{3}\right)=\left(\theta_{2}, \theta_{3},-\theta_{1}+\right.$ $\left.\theta_{2}\right)$. Then $\phi(0,0,0)=(0,0,0)$ and

$$
\phi_{*}=\left(\begin{array}{rrr}
0 & 1 & 0 \\
0 & 0 & 1 \\
-1 & 1 & 0
\end{array}\right)
$$

satisfies the hypothesis.]

Now let $e^{3} \subset T^{3}$ be a smooth closed 3-cell about $*$ and set $T_{0}^{3}=T^{3}-e^{3}$. Then since $\operatorname{det} A=-1$ by a further isotopy fixing $*$ we may assume $\phi_{0}$ : $T_{0}^{3} \rightarrow T_{0}^{3}, \phi_{0}=\phi \mid T_{0}^{3}$, is a diffeomorphism with $\phi_{0} \mid \partial e^{3}$ an orientation reversing orthogonal map. We now let $M_{0}=M_{0}(A)$ be the mapping torus of $\phi_{0}$. That is $M_{0}=T_{0}^{3} \times I /(X, 0) \sim\left(\phi_{0}(X), 1\right)$.

Note that $\partial M_{0}$ is, in fact, the nonorientable $S^{2}$-bundle over $S^{1}$ and so $\partial M_{0}=H$. Thus the construction of $Q$ is complete.

To construct $f:(X, \partial X) \rightarrow(Q, \partial Q)$ we must construct a map $h: M_{0} \rightarrow T_{C}$ such that $h \mid \partial M_{0} \rightarrow \partial T_{C}$ is the identity with $f=\mathrm{id}_{X_{0}} \cup h$ the requisite simple homotopy equivalence. Now a straightforward calculation using $\operatorname{det}\left(I-A^{2}\right)$ $= \pm 1$ shows that $M_{0}$ is a $\mathrm{Z}$-homology 1 -sphere and $T_{C}$ is homotopy equivalent to $S^{1}$. Thus the obstructions to extending the identity $\partial M_{0} \rightarrow \partial T_{C}$ to a map $h: M_{0} \rightarrow T_{C}$ all vanish and $h$ exists.

What must still be shown is

(1) $f$ is a simple homotopy equivalence;

(2) $f$ is not homotopic to a PL-homeomorphism.

The proof of (1) is a rather straightforward homotopy theoretic calculation. The proof of (2) uses the difference between the exotic framing on $T^{3}$ and the other framings to show that the 'PL normal invariant' of $f$ is nonzero and thus $f$ cannot be homotopic to the identity. We shall define 'normal invariants' and comment further on this proof in Chapter 6.

To prove the first part of the corollary (the last part will also be discussed in Chapter 6) it must only be shown that any homotopy equivalence of $\mathbf{R} P^{4}$ with itself is homotopic to the identity. But calculating $\left[R P^{4}, \mathbf{R} P^{4}\right]$ one finds any map inducing the identity on $\pi_{1}\left(\mathbf{R} P^{4}\right)$ is homotopic to the identity map. Thus the only homotopy equivalence in $\left[\mathbf{R} P^{4}, \mathbf{R} P^{4}\right]$ is the class of the identity map, and so the map $f$ constructed above cannot be a self-homotopy equivalence. $Q$ must in fact not be PL-homeomorphic to $\mathbf{R} P^{4}$. It is still possible that $Q$ is homeomorphic to $\mathbf{R} P^{4}$ since the possibility that $f$ is homotopic to a topological homeomorphism has not been ruled out. ( $f$ has zero topological normal invariants.) We note that different choices of $A$ give possibly different $Q$ 's. Thus there could conceivably be many distinct diffeomorphism classes of fake $\mathbf{R} P^{4}$ 's.

Now suppose $Q(A)$ is a fake $R P^{4}$. Let $\Sigma_{A}$ be its universal covering space. Then $\Sigma_{A}$ is a homotopy 4-sphere and one can ask whether it is homeomorphic to $S^{4}$. We note immediately that the existence of fake $\mathbf{R} P^{4}$ 's implies

THEOREM 4.6 [CS 2]. There is a smooth free involution on a homotopy 4-sphere $\Sigma^{4}$ which has no equivariant PL-homeomorphism with a linear action on $S^{4}$. 
In [AK 1] Kirby and Akbulut claimed to have shown that for at least certain matrices $A$, such that $Q(A)$ is a fake $\mathbf{R} P^{4}, \Sigma_{A}$ is PL homeomorphic to $S^{4}$. In particular, there would then have existed an 'exotic' involution on $S^{4}$ itself, rather than just on a homotopy 4-sphere $\Sigma^{4}$. The proof in [AK] is however wrong. (See [Rb].)

The key idea of that proof was an explicit construction of a handlebody picture for $\Sigma_{A}$ and the use of Kirby moves to show that $\Sigma_{A}$ is just $S^{4}$. We recall that $Q(A)$ was constructed by writing $X=\mathbf{R} P^{4}$ as the union of two pieces, $X_{0}=$ the normal $D^{2}$-bundle of $\mathbf{R} P^{2}$ in $\mathbf{R} P^{4}$ which we shall also denote by $\mathbf{R} P^{2} \widetilde{\times} D^{2}$ and $T_{C}$ the nontrivial $D^{3}$-bundle over $S^{1}$ written as $S^{1} \tilde{\times} D^{3}$.

Then $S^{1} \tilde{\times} D^{3}$ was replaced by $M_{0}=$ mapping torus of $\phi_{A} \mid T_{0}^{3}$. Thus the construction of $\Sigma_{A}$ is just $\Sigma_{A}=\widehat{R P^{2} \times B^{2}} \cup \tilde{M}_{0}$, where the $\sim$ 's indicate 2-fold cover. But $\widetilde{R P^{2} \widetilde{\times} B^{2}}$ is just $S^{2} \times B^{2}$ and $\tilde{M}_{0}$ is the mapping torus of $\phi_{A}^{2} \mid T_{0}^{3}$.

Let

$$
A=\left(\begin{array}{rrr}
0 & 1 & 0 \\
0 & 0 & 1 \\
-1 & 1 & 0
\end{array}\right)
$$

be the matrix of our previous example. Then a framed link picture of the mapping torus of $\phi_{A}^{2}$ is exhibited in Figure 4.6A. (A general procedure for constructing handlebody pictures of mapping tori of diffeomorphisms of 3-manifolds can be found in [Mont 2].) Finally, the $S^{2} \times B^{2}$ must be added.

This can be done in two distinct ways. (See the discussion in $\$ 7.4$ and the references there.) Unfortunately as J. H. Rubenstein noted [Rb] the way $S^{2} \times B^{2}$ was added to the link picture in 4.6A (see 4.6B) does not correspond to the universal covering space of $Q(A)$. Thus the link moves of [AK 2] do not prove that $\Sigma_{A}$ is homeomorphic to $S^{4}$. By choosing the correct gluing map for $S^{2} \times B^{2}$ one can in fact use link calculus techniques to construct a framed link picture of $\Sigma_{A}$ [AK 5]. However, despite a good deal of recent activity, it is as yet still unknown whether $\Sigma_{A}$ is or is not homeomorphic to $S^{4}$ and thus whether $S^{4}$ admits an 'exotic' involution.

The manifold $\Sigma$ pictured in Figure 4.6B, though not $\Sigma_{A}$, is nevertheless a homotopy 4 sphere and it is still instructive to recall how Akbulut and Kirby showed it to be homeomorphic $S^{4}$. One begins by sliding handles until all the 1-handles in this figure are cancelled by complementary 2-handles. This is indicated in Figure 4.7 for the one handle corresponding to the $0 \times B^{3} \cup \infty$ $\times B^{3}$ and in Figure 4.8 we show what is left after all the 1-handles are cancelled. However examining Figure 8 carefully we see that it is just the 3-component unlink and thus is complementary to the three 3-handles coming from the mapping torus of $\phi_{A}^{2} \mid T_{0}^{3}$. Thus what is represented by Figure 8 is precisely $S^{4}$ !

We note that the homotopy 4-spheres $\Sigma_{A}$ provide, at present, the most plausible candidates for counterexamples of the 4-dimensional Poincaré conjecture. We leave as a not unrewarding challenge to the reader to determine 
which, if any, of the $\Sigma_{A}$ 's are homeomorphic to $S^{4}$ or to show that some $\Sigma_{A}$ is, in fact, not homeomorphic to $S^{4}$.

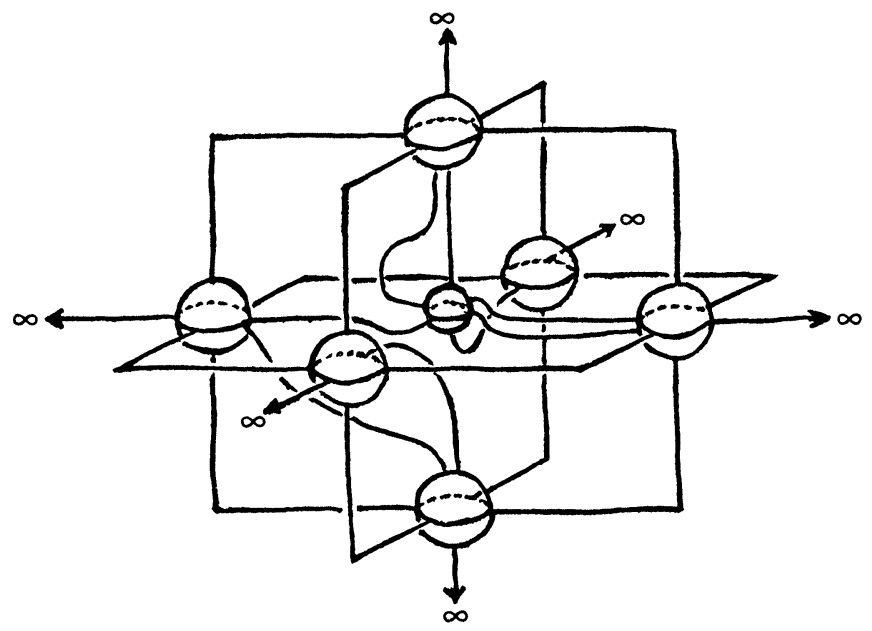

There is an additional ball centered at $\infty$ and connected to the other

1-handles in the picture by the link components with arrows pointing towards $\infty$.

FIGURE 4.6A

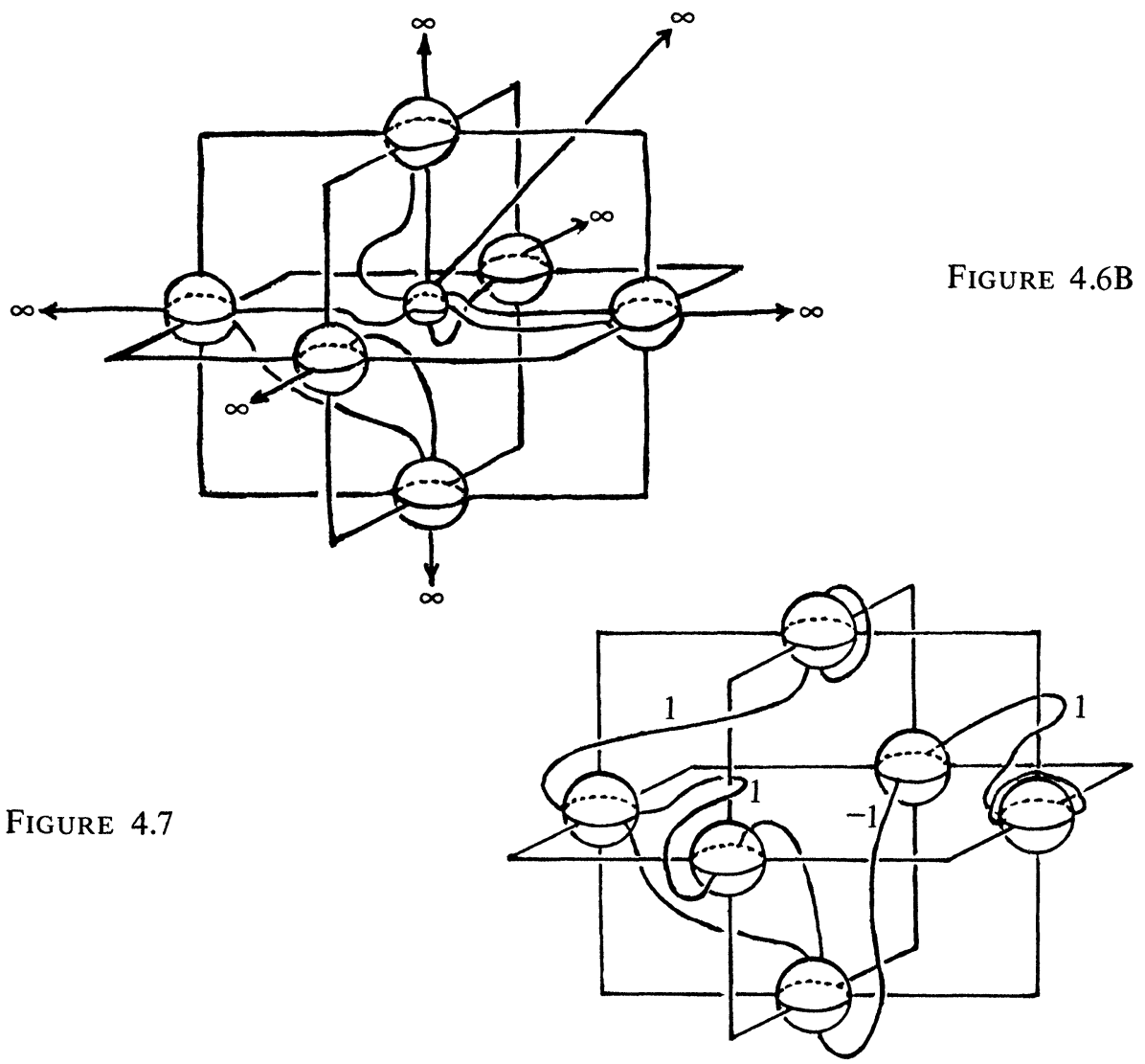




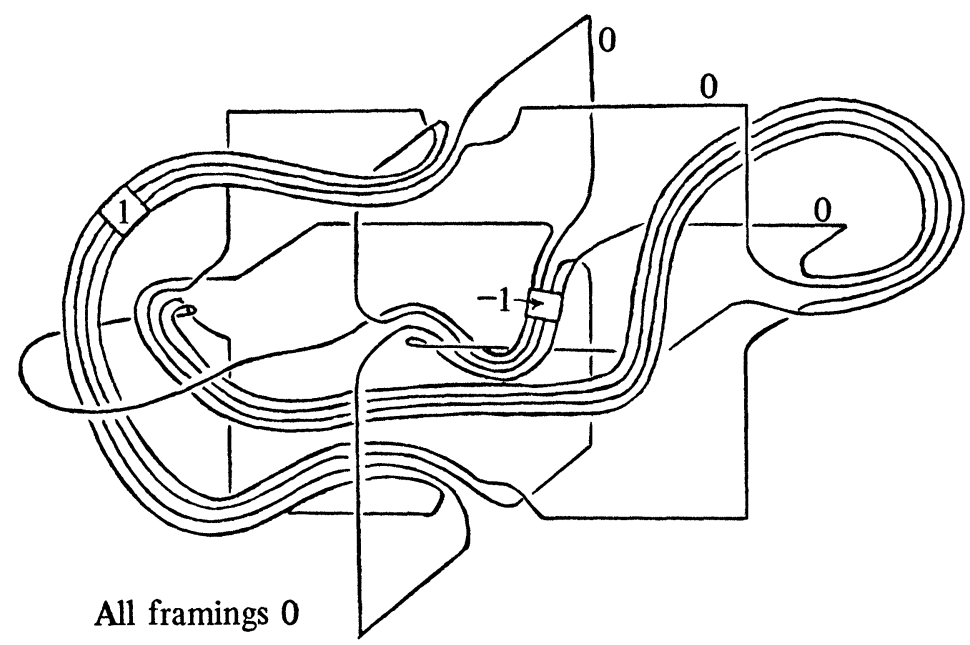

FIGURE 4.8

4.3 Triangulating high-dimensional manifolds. The relationship between 3and 4-manifolds captured by the $\mu$-invariant leads to interesting consequences for higher-dimensional manifold theory as well. Returning to the map $\mu$ : $\mathcal{H}^{3}(\mathbf{Z}) \rightarrow \mathbf{Z}_{2}$ let $\partial \mathcal{H}^{3}(\mathbf{Z})$ be the subgroup of elements of $\mathcal{H}^{3}(\mathbf{Z})$ which bound acyclic (PL) 4-manifolds. Since $\mu\left(\partial \mathcal{H}^{3}(\mathbf{Z})\right)=0$ letting $\theta_{3}^{H}=\mathcal{H}^{3}(\mathbf{Z}) / \partial \mathcal{H}^{3}(\mathbf{Z})$ we see that $\mu$ induces a homomorphism $\bar{\mu}: \theta_{3}^{H} \rightarrow \mathbf{Z}_{2}$ which remains onto. What is the structure of $\theta_{3}^{H}$ and the nature of $\bar{\mu}$ ? Essentially nothing other than the surjectivity of $\bar{\mu}$ is known. In particular it is not even known whether $\theta_{3}^{H}$ is finitely generated! Among the more well-known conjectures regarding $\theta_{3}^{H}$ are

CONJECTURE I. There exists an element of order 2 in $\theta_{3}^{H}$.

CONJECTURE II. Ker $\bar{\mu}=0$.

The extreme importance of $\theta_{3}^{H}$ and the significance of Conjecture I becomes apparent as a result of the following.

Theorem 4.7 (Galewski-Stern, See also Matumoto, [GS 1], [GS 2], [Mat]). Suppose $H$ is a Z-homology 3-sphere with $\mu(H) \neq 0$ such that $H \# H$ bounds an acyclic (PL) 4-manifold. Then every topological m-manifold $M$ ( $m \geqslant 5$ if $\partial M=\varnothing$ and $m \geqslant 6$ otherwise) can be triangulated as a simplicial complex!

Note that by the work of Kirby-Siebenmann there exist $m$-manifolds which cannot admit the structure of a PL-manifold. We already mentioned that $M_{1}^{4} \times S^{n}$ for $n \geqslant 1$ will be a topological manifold admitting no PLstructure [ $M_{1}^{4}$ as in Chapter 1]. These manifolds cannot be triangulated combinatorially. [We say a triangulation is combinatorial if it is PL-homogeneous. That is if $M$ is a manifold with triangulation $K$ (so $|K|=M$ ) then for $K$ to be a combinatorial triangulation we must have that for every $x, y \in M$ there exists a piecewise-linear homeomorphism $h$ (PL relative to $K$ of course) such that $h(x)=y$.] However, they still might have noncombinatorial triangulations. (See [Sb 2].) [That not all triangulations are combinatorial was demonstrated by R. D. Edwards [Ed 1], [Ed 2] who showed that $S^{n}(n \geqslant 5)$ 
always has noncombinatorial triangulation. (Proof: We have $\Sigma^{n-3} P$ is topologically homeomorphic to $S^{n}$ and clearly the triangulation of $S^{n}$ defined by $\Sigma^{n-3} P$ is not combinatorial)].)

We note that the problem of the structure of $\theta_{3}^{H}$ is unique to the relationship between 3- and 4-manifolds in the following sense.

Let $\mathcal{H}^{4 k-1}(\mathrm{Z})$ be the group of (DIFF) $h$-cobordism classes of (DIFF) Z-homology spheres which bound almost parallelizable smooth $4 k$-manifolds and let $\partial \mathcal{H C}$ be the subgroup bounding smooth contractible $4 k$-manifolds. Let $\boldsymbol{\theta}_{4 k-1}^{H}$ be $\mathcal{H}^{4 k-1}(\mathbf{Z}) / \partial \mathcal{F}^{4 k-1}$. Then there exists an isomorphism $\lambda: \boldsymbol{\theta}_{4 k-1} \rightarrow \mathbf{Z}_{t}$ (where $t=I_{k}, I_{k}$ as in Theorem 1.15) and $\lambda[M]=\sigma\left(W_{M}\right) \bmod t, W_{M}$ an almost parallelizable $4 k$-manifold with $\partial W_{M}=M$. (See [M 2].)

\section{CHAPTER 5. SURGERY THEORY AND ITS APPLICATIONS}

5.1 Surgery theory in higher-dimensions. Throughout many of the preceding sections of this paper we have alluded to surgery and its implications. In particular there are the fundamental results of $[\mathrm{Br} \mathbf{1}]$, [N] on the classification of manifolds of a given homotopy type which we mentioned in Chapter 1 and the surgery-type calculations used to show that the homotopy $\mathbf{R} P^{4}$ constructed in [CS 2] is indeed fake. Unfortunately the full power of higher-dimensional surgery theory is not available in low-dimensional topology. In the following chapters we will illustrate what goes wrong in dimension 4 (and 5) and present some examples of what can be salvaged. In order to do this we use this chapter to review some of the basic ideas, methods and constructions of high-dimensional surgery theory.

We begin by reviewing the essential ideas of surgery theory. Thus let us consider the following questions. (We assume that we are working in some fixed category either DIFF or PL or TOP.)

(1) Suppose $X$ is a CW-complex, $M^{m}$ a manifold and $\phi: M^{m} \rightarrow X$ a map. When can we modify $M$ to get a new manifold $M^{\prime}$ and map $\phi^{\prime}: M^{\prime} \rightarrow X$ with $\phi^{\prime}$ a (simple) homotopy equivalence?

(2) Suppose $M, M^{\prime}$ are manifolds and $f: M \rightarrow M^{\prime}$ is a (simple) homotopy equivalence. When is $f$ homotopic to an isomorphism?

The basic geometrical construction used in solving these problems is the same, that of surgering $M$. We recall its definition: [M 3], [KM 2].

Suppose $f: S^{r} \times D^{m-r} \rightarrow V^{m}$ is an orientation preserving embedding into the oriented manifold $V^{m}$. (We will in the sequel work in the smooth category. Analogous results occur in the PL locally flat and TOP locally flat categories.) Form the disjoint sum $V^{m}-f\left(S^{r} \times 0\right) \amalg D^{r+1} \times D^{-r-1}$ and let $V^{\prime}$ be the quotient manifold obtained by identifying $f(u, r v)$ with $(r u, v)$ for $u \in S^{r}, v \in S^{m-r-1}$ and $0<r<1$. We say $V^{\prime}$ is obtained by surgery on an $r$-sphere in $V$ and write $V^{\prime}=\chi(V, f)$ to indicate the dependence on $f$ in this process. An alternative way, already mentioned in Chapter 1 , to visualize $V^{\prime}$ is as follows. Let $W=V \times I$ and attach an $(r+1)$-handle $H^{r+1}$ to the cobordism $W$ by means of the attaching map $f: S^{r} \times D^{m-r} \rightarrow V^{m} \times\{1\}$. This gives a new cobordism $W^{\prime}=V \times I \cup H^{r+1}$ with ends $V$ and $\tilde{V}=\partial W^{\prime}$ - $V$. It is readily seen that $\tilde{V}$ is diffeomorphic to $V^{\prime}$ defined above and thus $V^{\prime}$ is of course cobordant to $V$. It is thus clear that talking about a surgery on 
$V$ is equivalent to talking about a handle addition to the cobordism $W=V$ $\times I$. We will say an embedding $f: S^{r} \times D^{m-r} \rightarrow V$ represents the homotopy class $\lambda \in \pi_{r}(V)$ if $\lambda=f_{*}(\theta), \theta$ the generator of $\pi_{r}\left(S^{r} \times D^{m-r}\right)$. One can then show that

LemMa 5.1 (MilnoR) [M 3]. If $f: S^{r} \times D^{m-r} \rightarrow V^{m}$ represents $\lambda \in \pi_{r}(V)$ and $m \geqslant 2 r+2$ then if $V^{\prime}=\chi(V, f)$ we have

(1) $\pi_{i}\left(V^{\prime}\right) \approx \pi_{i}(V), i<r$,

(2) $\pi_{r}\left(V^{\prime}\right) \approx \pi_{r}(V) / \Lambda(\lambda)$ where $\Lambda(\lambda)$ is a certain subgroup of $\pi_{r}(V)$ containing $\lambda$.

Thus surgery as above kills the element $\lambda$ in $\pi_{r}\left(V^{m}\right)$.

To apply our construction to the solution of problem 1 suppose $\phi: V \rightarrow X$ is a map of the manifold $V^{m}$ to the CW-complex $X$. Suppose also that $\phi_{*}$ : $\pi_{*}(V) \rightarrow \pi_{*}(X)$ is onto. We can then try to modify $\phi$ and $V$ to get a homotopy equivalence by killing the homotopy classes in $\operatorname{Ker} \phi_{*}$ ! In fact $\phi$ is a homotopy equivalence if and only if $\phi_{*}$ is an isomorphism [Spr 1] and using Poincaré duality this is guaranteed by $\operatorname{Ker} \phi_{*}^{i}=0$ for $i \leqslant[\operatorname{dim} V / 2]$. More precisely if $f: S^{r} \times D^{m-r} \rightarrow V^{m}$ is an embedding and $f_{0}=f \mid S^{r} \times 0$ then letting $W^{\prime}$ be the cobordism $V \times I \cup_{f} H^{r+1} ; H^{r+1}=D^{r+1} \times D^{m-r}=$ an $(r+1)$-handle attached by $f$; we see that $\phi$ is homotopically extendible to a map $\phi^{\prime}: W^{\prime} \rightarrow X$ (and thus homotopic to a map $\phi_{1}=\left.\phi^{\prime}\right|_{+} W^{\prime}$ of $\chi(V, f) \rightarrow$ $X$, where $\left.\partial_{+} W^{\prime}=\partial W^{\prime}-V \times\{0\}=\chi(V, f)\right)$ if and only if $\phi \circ f_{0}$ is null homotopic. We are thus led to define the homotopy groups of $\phi$.

Definition 5.2. Suppose $\phi$ is on $M$ to $X$.

Let $(f, g) f: S^{k} \rightarrow M, g: D^{k+1} \rightarrow X$, be a pair of maps such that $g i=\phi f$ where $i: S^{k} \rightarrow D^{k+1}$ is the canonical inclusion. If $\left(f^{\prime}, g^{\prime}\right)$ is another such pair say that $\left(f^{\prime}, g^{\prime}\right) \sim(f, g)$ if and only if their exist homotopies $f_{t}: S^{k} \rightarrow M, g_{t}$ : $D^{k+1} \rightarrow X$ with $g_{t} i=\phi f_{t}$ such that $\left(f_{0}, g_{0}\right)=(f, g)$ and $\left(f_{1}, g_{1}\right)=\left(f^{\prime}, g^{\prime}\right)$.

Let $\pi_{k+1}(\phi)$ be the set of equivalence class of pairs $(f, g)$ with the obvious group structure.

Thus our surgery problems in this case is a question of determining when we can surger elements $\alpha \in \pi_{k+1}(\phi)$.

Notice that if $\alpha$ is representable by an embedding $f: S^{r} \times D^{m-r} \rightarrow M$ then since $S^{r} \times D^{m-r}$ is parallelizable we must have that $f^{*} T_{M}=f_{0}^{*} T_{M}$ is trivial ( $T_{M}=$ tangent bundle of $M$ ). To guarantee this, since $\phi \circ f_{0}$ is null homotopic ( $f$ represents $\alpha \sim\left(f_{0}, g\right), g$ a null homotopy of $\phi \circ f_{0}$ ) it suffices to know that $T_{M}$ is induced by $\phi$ from some bundle over $X$. Actually for technical reasons it is more convenient to work with stable normal bundles rather than tangent bundles. (Recall that we can think of the stable normal bundle $\nu_{M}^{s}$ of a manifold $M^{m}$ as the normal bundle $\nu^{k}$ of any embedding $M^{m} \rightarrow S^{m+k}$, $k \gg m . T_{M}$ is stably trivial if and only if $\nu^{s}$ is trivial since $T_{M} \oplus \nu^{k}=T_{S^{m+k} \mid M}$ and $T_{S^{m+k}} \oplus e$ ( $e$ the trivial line bundle) is always trivial [M 3], [MK 2].)

Note also that the analogue of the stable normal bundle exists in the categories PL, TOP, $G$ as well as in DIFF.

Thus our surgery problem can be recast in the following form.

Definition 5.3. Let $X$ be a CW complex and $\xi^{k}$ a linear bundle (in the appropriate category) over $X$. Then a normal map into $\left(X, \xi^{k}\right)$ is a triple $\left(M^{m}, f, b\right)$ where $M^{m}$ is an $m$-dimensional manifold (in DIFF, PL or TOP 
depending on which fixed category we are working in), $f: M^{m} \rightarrow X$ a map and $b: \nu^{k} \rightarrow \xi^{k}$ a linear bundle map covering $f$ where $\nu^{k}$ is the normal bundle of $M^{m} \rightarrow S^{n+k}, k \gg m$. (Similarly $(f, b):(M, \partial M) \rightarrow(X, Y)$ is a normal map of pairs if $(X, Y)$ are a $C W$ pair of dimension $m$ and $f, b$ are as above with $\nu^{k} \mid \partial M=$ normal bundle of $\partial M$ in $S^{m+k-1}$.)

We will consider normal maps $\left(M_{1}, f_{1}, b_{1}\right),\left(M_{0}, f_{0}, b_{0}\right)$ equivalent [written $\left.\left(f_{1}, b_{1}\right) \sim\left(f_{0}, b_{0}\right)\right]$ if there exists a cobordism $W \subset S^{m+k} \times I$ with $\partial W=$ $\partial_{+} W \cup \partial_{-} W=M_{1} \cup M_{0}$, a map $F: W \rightarrow X$ with $F \mid M_{i}=f_{i}$ and a linear bundle map $B: \omega \rightarrow \xi^{k}$ covering $F$, where $\omega$ is the normal bundle of $W \subset$ $S^{m+k} \times I$ and $B \mid \nu_{i}=b_{i}$. We call $(W, F, B)$ a normal cobordism. (If we have normal maps $\left(f_{i}, b_{i}\right) f_{i}:\left(M_{i}, \partial M_{i}\right) \rightarrow(X, Y)$ we can define a normal cobordism entirely analogously noting that in this case $\partial W^{m+1}=\partial_{+} W \cup U^{m+1} \cup$ $\partial_{-} W, \partial_{+} W=M_{1}, \partial_{-} W=M_{0}$ and $\partial U^{m+1}=\partial M_{1} \cup \partial M_{0}$ (i.e., $U^{m+1}$ is a cobordism of the boundary). If $U^{m+1}$ is a product we say $(W, F, B)$ is a cobordism rel $\partial$ and we demand this in all future relative definitions.)

We let $\Omega_{m}(X, \xi)$ be the set of equivalence classes $\left(\Omega_{m}(X, Y ; \xi)\right.$ in the relative case) $\Omega_{m}(X, \xi)\left(\Omega_{m}(X, Y ; \xi)\right)$ has a group structure induced by disjoint union and is called the $m$ th bordism group associated with $(X, \xi)$. (Henceforth we will indicate notation explicitly only for the absolute case and leave it to the reader to fill in the relative case.)

Now given a normal map $(f, b): M \rightarrow X$ Question 1 then becomes:

$1^{\prime}$ : Does the bordism class $[(f, b)]$ defined by $(f, b)$ contain a (simple) homotopy equivalence?

Clearly if $X$ were to be homotopy equivalent to a manifold $M$ it would have to satisfy the analogue of Poincaré duality (where if $M$ is nonorientable we still have Poincaré duality if we use twisted coefficients (see [Wa 3, Chapter 2] for details)). CW complexes satisfying a sort of Poincaré duality condition are called Poincaré complexes and the condition that $X$ be a Poincaré complex is then clearly a necessary one if we wish to solve $1^{\prime}$. In the interest of simplicity we henceforth assume that $X$ is in fact a topological manifold referring the reader to [Br 1], [Wa 3] for a discussion of Poincaré complexes and the necessary modifications there.

Furthermore, it is a straightforward verification that if $(f, b)$ is normally cobordant to $\left(f^{\prime}, b^{\prime}\right)$ then the degree of $f$ equals the degree of $f^{\prime}$. Since a homotopy equivalence has degree one we add this condition to the normal maps we are willing to consider.

We let $\Omega_{m}^{+}(X, \xi) \subset \Omega_{m}(X, \xi)$ then denote the degree 1 normal maps and note that $\Omega_{m}^{+}$is a coset (rather than subgroup) of $\Omega_{m}(\gamma, \xi)$.

Actually we are generally interested in the existence of any isomorphism (or simple homotopy equivalence) $f: M \rightarrow X$, rather than only one which pulls back a specific bundle $\xi$ over $X$. Thus we let $\mathcal{T}_{m}(X)$ be the union of all the $\Omega_{m}^{+}(X, \xi)$ over all $k$-plane bundles $\xi$ over $X(k \gg m)$ modulo the additional equivalence relation that $\left(f_{0}, b_{0}\right) \in \Omega_{m}^{+}\left(X, \xi_{1}\right)$ is equivalent to $\left(f_{1}, b_{1}\right) \in$ $\Omega_{m}^{+}\left(X, \xi_{2}\right)$ if and only if $\left(f_{0}, b_{0}\right)$ is normally cobordant to $\left(f_{1}, a b_{1}\right)$ for some linear bundle automorphism $a: \xi_{1} \rightarrow \xi_{0}$. (The reason for the terminology $\mathcal{T}_{m}(X)$ will be mentioned later.) If we wish to emphasize which category we are working with we use $\mathcal{T}_{m}^{H}(X)$ with $H=$ DIFF, PL or TOP. The equivalence class of a normal map (of degree 1) in $\sigma(X)$ is called its normal (or 
splitting) invariant. (In the relative case $\mathcal{T}(X, \partial X)$ we include the condition that our normal maps $(f, b) \in \mathcal{T}(X, \partial X)$ represented by $f:(M, \partial M) \rightarrow$ $(X, \partial X)$ induces a (simple) homotopy equivalence when restricted to $\partial M$.)

We can now reformulate $1^{\prime}$ as

$1^{\prime \prime}:$ Given a bordism class $\alpha \in \mathcal{T}_{m}(X)$, classify those of its members $(f, b)$ for which $f$ is a (simple) homotopy equivalence.

Actually it suffices to find some member $\left(f_{0}, b_{0}\right)$ which is a (simple) homotopy equivalence. Then we can study its uniqueness as follows.

Suppose $\left(f_{0}, b_{0}\right): M_{0} \rightarrow X,\left(f_{1}, b_{1}\right): M_{1} \rightarrow X$ are both simple homotopy equivalences in $\alpha$. Then the normal cobordism $(F, B): W \rightarrow X \times[0,1]$ between them is a normal map of degree 1 and we can consider the problem of modifying $(F, B)$ keeping $(\partial F, \partial B)$ fixed. If $(F, B)$ can be surgered into a simple homotopy equivalence one would conclude that $\left(f_{0}, b_{0}\right)$ is $s$-cobordant to $\left(f_{1}, b_{1}\right)$ and if $m \geqslant 5(m \geqslant 6$ in the relative case $\partial M \neq \varnothing)$ one could conclude by the $s$-cobordism theorem that $\left(f_{0}, b_{0}\right)$ is unique up to isomorphism.

Note that our discussion of the question of the uniqueness of $\left(f_{0}, b_{0}\right)$ above applies equally well to the question of when a (simple) homotopy equivalence is homotopic to an isomorphism. That is if $(f, b)$ is a normal map which is a (simple) homotopy equivalence one can: (1) examine the elements of Isom $(M)$ and see whether any of them is normally cobordant to $(f, b)$ and (2) if $\left(f^{\prime}, b^{\prime}\right)$ is an isomorphism normally cobordant to $(f, b)$ one can use surgery theory as above to measure the obstruction to making the normal cobordism into an $s$-cobordism and thus (in the appropriate dimensions) establishing that $f$ is homotopic to an isomorphism.

We are thus led to define the set of (simple) homotopy structures on $X, \mathcal{S}(X)$ (or $\mathcal{S}^{\mathrm{DIFF}}, \mathcal{S}^{\mathrm{PL}}, \mathcal{S}^{\mathrm{TOP}}$ if we wish to emphasize the category involved) as the set of equivalence classes of maps $\phi: M \rightarrow X$, where $M$ is a compact manifold, $\phi$ a (simple) homotopy equivalence and $\left(M_{1}, \phi_{1}\right)$ is equivalent to $\left(M_{2}, \phi_{2}\right)$ if there is an $s$-cobordism $W$ between $M_{1}$ and $M_{2}$ and a simple homotopy equivalence $F: W \rightarrow X \times[0,1]$ with $F \mid M_{i}=\phi_{i}$. (In the relative case we demand that $\phi_{i} \mid \partial M_{i}$ is already an isomorphism and $W$ is already a product between $\partial M_{1}$ and $\partial M_{2}$.) Clearly the item of interest in our analysis is then $\delta(X)$ (or $\delta(X, \partial X)$ ). Note that if $\phi: M \rightarrow X$ represents an element of $\delta(X)$ there exists an obvious (forgetful) map $\eta: \delta(X) \rightarrow \sigma(X)$ associating to $\phi$ the class of $\left(\phi, \phi^{*}\right)$ in $\mathcal{T}(X)$, where $\phi^{*}$ is the obvious map on stable normal bundles induced by $\phi$. (If $X$ is not a manifold we still have an analogue of a normal bundle, the Spivak normal fiber space $\nu_{X}$ as defined in $[\mathrm{Br} \mathbf{1}]$ or $[\mathrm{Wa}$ 3].)

A moment's reflection now shows that the basic problem of surgery theory is essentially the question of understanding $\operatorname{Ker} \eta$ and $\operatorname{Im} \eta$. To this end we begin by giving an alternative approach to $\mathscr{T}(X)$ due to Sullivan ([S 1], [S 2], see also [Wa 3]).

We first review some bundle theory. We recall that in the classification theory of smooth vector bundles over a locally finite $\mathrm{CW}$ complex $X$, there exist universal classifying spaces $\mathrm{BO}_{(k)}$ and bundles $\gamma_{0}^{k}$ for $k$-plane bundles such that isomorphism classes of bundles over $X$ are in 1-to-1 correspondence with the homotopy classes, $\left[X, \mathrm{BO}_{(k)}\right]$; with the class represented by $f$ : 
$X \rightarrow \mathrm{BO}_{(k)}$ corresponding to the bundle $f^{*} \gamma_{0}^{k}$. Letting $\mathrm{BO}=\cup_{k} \mathrm{BO}_{(k)}$ and noting the exact sequence $0 \rightarrow \mathrm{BO}_{k} \hookrightarrow \mathrm{BO}_{k+1}$ we similarly have a classifying space for stable vector bundles over $X$. One can make similar construction in the PL and TOP categories and thus define spaces $B_{\mathrm{PL}(k)}, B_{\mathrm{PL}}$ and $B_{\mathrm{TOP}(k)}$, $B_{\text {TOP }}$ with corresponding universal bundles $\gamma_{\mathrm{PL}}^{k}, \gamma_{\mathrm{PL}}, \gamma_{\mathrm{TOP}}^{k}, \gamma_{\mathrm{TOP}}$. (We are using the natural identification $B_{\mathrm{DIFF}(k)}$ with $B_{\mathrm{O}(k)}, \mathrm{O}(k)$ the orthogonal group of $k \times k$ matrices, induced by $\operatorname{DIFF}(k) \simeq \mathrm{GL}(k) \simeq \mathrm{O}(k)$. (See [KS 2].)

The corresponding bundles in PL or TOP are not actually vector bundles but behave analogously [see KS 1]. We shall refer to all such vector-like bundles simply as $H$-bundles in what follows. $(H=\mathrm{PL}, \mathrm{TOP}$ or $\mathrm{DIFF} \simeq 0$.)

We need a corresponding concept in the homotopy theory of manifolds. Thus following [ $\mathrm{Br} 1]$ let $G(k)$ be the space of autohomotopy equivalences of $S^{k-1}$. Then there exists classifying spaces $B_{G(k)}$ and universal $(k-1)$ spherical fibrations $\gamma_{k}$ over $B_{G(k)}$ such that if $X$ has the homotopy type of a locally finite $\mathrm{CW}$ complex then fiber homotopy equivalence classes of $(k-$ 1)-spherical fibrations over $X$ are in 1-to-1 correspondence with $\left[X, B_{G(k)}\right]$. One can also define $B_{G}, \gamma^{S}$ as universal objects for stable spherical fibrations over $X$.

Now by a result of Spivak (see $[\mathrm{Br} 1]$ ), if $X$ is a Poincaré complex of dimension $m$, then there exists a $(k-1)$-spherical fibration $\nu_{X}^{k}$ over $X$ $(k>m+1)$, unique up to suspension and stable fiber homotopy equivalence, such that any class in $\Omega_{m}^{+}(X, \xi)$ induces a stable fiber homotopy equivalence of $\xi$ on $\nu_{X}^{k}$, unique up to fiber homotopy. Furthermore the set of such classes map bijectively to the set of stable fiber homotopy equivalences of $\xi$ on $\nu_{X} \cdot \nu_{X}^{k}$ is called the Spivak normal fiber space of $X$.

We can now consider $\mathscr{T}_{M}(X)$ as a set of pairs $(\xi, h)$, with $\xi$ as above and $h$ : $\xi \rightarrow \nu_{x}$ a (stable) fiber homotopy equivalence of $\xi$ and $\nu_{X}$, modulo the appropriate equivalence relation.

To clarify this notion further consider the commutative diagram of maps of classifying spaces.

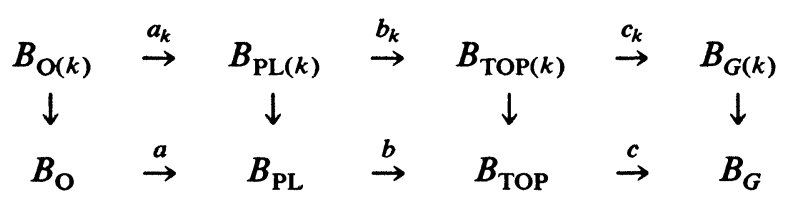

where

$a_{k}$ corresponds to triangulating the bundle,

$b_{k}$ is the forgetful functor from PL bundles to TOP bundles,

$c_{k}$ is the functor from TOP bundles to spherical fibrations obtained by deleting the zero section with $a, b, c$ induced by limit maps (nonunique).

The maps $a_{k}, b_{k}, c_{k}$ and $a, b, c$ can be considered fibrations and thus have fibers which we denote by $\mathrm{PL}(k) / \mathrm{O}(k), \operatorname{TOP}(k) / \mathrm{PL}(k), G(k) / \mathrm{TOP}(k)$ and $\mathrm{PL} / \mathrm{O}, \mathrm{TOP} / \mathrm{PL}, G / \mathrm{TOP}$, respectively. By composition we can also define natural fibrations $B_{\mathrm{O}} \rightarrow B_{G}, B_{\mathrm{PL}} \rightarrow B_{G}$ and thus obtain fibers $G / \mathrm{O}, G / \mathrm{PL}$ also.

Then a pair $(\nu, h)$ as above corresponds to a reduction of the structural group of $\nu_{X}$ from $G$ to $O$ (or PL or TOP) and such reductions correspond 
bijectively to homotopy classes of sections of the associated bundle with fiber $G / H(H=$ O, PL, or TOP $)$.

We can thus view pairs $(\nu, h)$ as defining $G / H$ structures on $\nu_{X}$. We call two such pairs $\left(\nu_{i}, h_{i}\right)$ equivalent if there is a $G / H$ bundle structure $(\tilde{\nu}, H)$ on $\nu_{X} \times I$ over $X \times I$ and equivalences $b_{i}: \tilde{\nu} \mid X \times i \rightarrow \nu_{i}$ with $b_{i}\left(H \mid \nu_{X} \times i\right)=\alpha_{i}$, $i=0,1$. We call such equivalence classes of pairs $(\nu, h)$ tangential structures on $X$. (This is the motivation behind the notation $\mathscr{T}_{H}(X)$.) One can then show that the fibration $G / H \rightarrow B_{H} \rightarrow B_{G}$ acts homotopically like a principal fibration so that the existence of a cross section of a $G / H$ bundle means that the bundle is homotopically just $X \times G / H$. Thus, given the existence of some tangential structure on $\nu_{X}$, we find that equivalence classes of tangential structures on $\nu_{X}$ correspond bijectively to the homotopy classes of map $[X, G / H]$.

Putting all this information together we arrive at

THEOREM 5.4. Let $X$ be an $H$-manifold ( $H=\mathrm{DIFF}$, PL or TOP). Then there is a bijection between $\sigma_{H}(X)$ and $[X, G / H]$ (in the relative case between $\mathcal{T}_{H}(X, \partial X)$ and $\left.\left[(X, \partial X),\left(G / H,{ }_{*}\right)\right]\right)$.

We can therefore compute $\mathscr{T}_{H}(X)$ by computing $\pi_{*}(G / H)$ and then trying to use obstruction theory to compute $[X, G / H]$. (For results on $\pi_{*}(G / H)$ and computations of this sort see [KS 1] and the references listed there. In particular for $H=$ PL [S 1], [S 2] or TOP [KS 1], the homotopy of $G / H$ is pretty well understood, though for $H=O$ much less is known.)

Now let us consider the problem of finding the image of $\eta$. That is the question of when is a normal map $(f, b)$ normally cobordant to a simple homotopy equivalence. Using the constructions of surgery theory elucidated above one can find an invariant, the surgery obstruction $\sigma(f, b)$, which solves this problem. $\sigma(f, b)$ lies in the Wall group $L_{m}^{s}(\pi, w), m=\operatorname{dim} X, \pi=\pi_{1}(X)$, $w: \pi \rightarrow\{ \pm 1\}$ the orientation character of $\pi$ (i.e. first Steifel-Whitney class of $X$ ). (The construction of $\sigma(f, b)$ is due to Browder and Novikov (see [Br 1] and [N] if $\pi=\{1\}$, and Wall [Wa 3] in general). In our discussion of $\eta$ we shall follow almost verbatim the treatment by Shaneson in [Sh 4]. See also [Lees] and [Wa 3].)

We begin by recalling (see [Sh 4]) an algebraic criterion for recognizing a (simple) homotopy equivalence.

THEOREM 5.5. Let $h:(M, \partial M) \rightarrow(X, Y)$ be a map between the manifold $M$ and the Poincare pair $(X, Y)$ such that $h \mid \partial M$ is a (simple) homotopy equivalence. Then $h$ is a homotopy equivalence if and only if

(1) $h_{*}: \pi_{1}(M) \rightarrow \pi_{1}(X)$ is an isomorphism,

(2) $h_{*}: H_{i}(M ; \mathbf{Z} \pi) \rightarrow H_{i}\left(X ; \mathbf{Z}_{\pi}\right)$ is an isomorphism for $i<[n / 2], n=$ $\operatorname{dim} X$, where $\pi=\pi_{1}(X)$ and $\mathbf{Z} \pi$ is the group ring of $\pi$.

(Recall that $H_{*}\left(X ; \mathrm{Z}_{\pi}\right)=H_{*}(\tilde{X} ; \mathrm{Z})$, where $\tilde{X}$ is the universal covering space of $X$.) Also the homotopy equivalence $h$ will be simple if its torsion invariant $\mathscr{T}(h)$ vanishes in the Whitehead group $\mathrm{Wh}(\pi)$. (See [M 5] for details.) 
Now a theorem of Smale [Sm] (see also [M 4]) tells us that any normal cobordism, relative to the boundary if there is any, can be obtained by a sequence of handle attachments along embedded copies of $S^{i} \times D^{n-i}$ (in the interior) that represent elements in the kernel of the map on $i$ th homotopy groups induced by the normal map. Thus, all normal maps normally cobordant to a given one, $(h, c)$ say, are obtained from it by "surgery" ([M 3], [N], [Br 1], [Wa 3]). On the other hand, it is easy to see that by surgery one may at least obtain that $h$ induces an isomorphism of fundamental groups and of the homology groups $H_{i}(M ; \mathrm{Z} \pi)$ with $H_{i}(X ; \mathrm{Z} \pi)$ for $i<[n / 2]$; i.e. to obtain $h$ that is $[n / 2]$-connected.

Suppose $n=2 k \geqslant 6$ is even. Let $K$ be the kernel of $h_{*}: H_{k}\left(M ; \mathbf{Z}_{\pi}\right) \rightarrow$ $H_{k}(X ; \mathbf{Z} \pi)$. By Poincaré duality, $K$ is a stably based $\mathbf{Z}$-module (denoted $K_{k}(M)$ in [Wa 3]). By the Hurewicz theorem, every element of $K$ is represented by a sphere $\alpha: S^{k} \rightarrow M$. Using transversality ("general position") one may define a nonsingular $(-1)^{k}$-Hermitian form $\phi: K \times K \rightarrow Z_{\pi}$, and a self-intersection form to which $\phi$ is associated, $\mu: K \rightarrow \mathbf{Z}_{\pi} /\left\{x-(-1)^{k} \bar{x}\right\}$, as done in [Wa 3], [Wa 4]. (Actually $\phi$ is purely homological, but $\mu$ requires some extra care. Here if $x=\Sigma \alpha_{g} g, \bar{x}=\Sigma w(g) \alpha_{g} g^{-1}$.) By $(-1)^{k}$-Hermitian, we mean that the form is sesquilinear and satisfies $\phi(x, y)=(-1)^{k} \phi(\overline{y, x})$. Also, $\mu(\alpha x)=\alpha \mu(x) \bar{\alpha}, \alpha \in \mathbf{Z} \pi, \phi(x, x)=\mu(x)+(-1)^{k} \mu(\bar{x})$, and $\mu(x+y)-\mu(x)$ $-\mu(y) \equiv \phi(x, y)$. Finally, nonsingularity in this context means that

$$
A \phi: K \rightarrow \operatorname{Hom}_{\mathbf{Z} \pi}\left(K, \mathbf{Z}_{\pi}\right)=K^{*},
$$

given by $A \phi(x)(y)=\phi(x, y)$, is an isomorphism of stably based modules, where the dual module $\left(\alpha \lambda(x)=\lambda(\bar{\alpha} x)\right.$ for $\left.\lambda \in K^{*}\right)$ has the dual class of stable bases.

Now, suppose $(K, \phi, \mu)$ happens to be hyperbolic; i.e., $K=L \oplus L^{\prime}, L$ and $L^{\prime}$ stably based modules of one-half the rank of $K$ whose stable bases "add up" to a stable base of $K$, with $\phi \mid L \times L=0$ and $\mu \mid H=0$. The module $L$ is called a subkernel. After stabilizing, which may be geometrically realized by taking the connected sum with $S^{k} \times S^{k}$ sufficiently many times, we may assume stably based modules are based and choose representative bases. (Also, we could choose $L^{\prime}$ with $\phi$ and $\mu$ trivial on $L^{\prime}$; see [Wa 3, \&5].) The Whitney trick for removing intersections and self-intersections implies that the basis for $L$ can be represented by $r$ disjointly embedded framed spheres $S_{i}^{k} \times D^{k}$ in $M$. Now an argument of Kervaire and Milnor [KM 2, \$7] for $\pi=\{e\}$ and Wall [Wa 3, §5] in the general case shows that performing surgery on these classes "kills" the kernel of $h_{*}$ so as to produce (see Theorem 5.5) a simple homotopy equivalence.

Thus we define the Wall group $L_{2 k}^{s}(\pi, w)$ as the Grothendieck group of forms $(K, \phi, \mu)$ as above, modulo the subgroups generated by hyperbolic forms. The preceding discussion can be formalized to give the following result of Wall [Wa 3, §5], at least for $n$ even.

THEOREM 5.6. Let $(h, b), h:\left(M^{n}, \partial M\right) \rightarrow\left(X^{n}, \partial X\right)$ be a normal map that induces a homotopy equivalence of boundaries. Assume $n \geqslant 5$. Then the invariant $\sigma(h, b) \in L^{s}(\pi, w)$ vanishes if and only if $(h, b)$ is normally cobordant relative to the boundary to a simple homotopy equivalence. 
Thus one has the sequence

$$
\mathcal{S}_{H}(x) \stackrel{\eta}{\rightarrow}[X ; G / H] \stackrel{\sigma}{\rightarrow} L_{n}^{s}(\pi, w), \quad n=\operatorname{dim} X \geqslant 5,
$$

$X$ a closed $H$-manifold.

Note. The definition of $L_{2 k}^{s}(\pi, w)$, as the definition of odd Wall groups to be indicated below, is given in its most primitive form. See [Wa 4] and [Ran] for more sophisticated and general definitions. (See also [Lees], [Sh 4].)

For $n=2 k+1 \geqslant 5$, Theorem 5.6 and the exact sequence (*) are also valid. In this case Kervaire and Milnor [KM] showed directly that, in the simply-connected case they studied, one could always perform surgery to obtain a homotopy equivalence. A satisfactory invariant was first introduced by Wall [Wa 3].

To describe the odd dimensional case, let $(h, b)$ be a normal map, $h$ : $(M, \partial M) \rightarrow\left(X^{2 k+1}, \partial X\right)$, assumed to induce a simple homotopy equivalence of boundaries. (Again, only a simple homology equivalence with coefficients in $\mathbf{Z} \pi_{1} X$ is really needed; see [CS 1].) Again we suppose $h$ induces isomorphisms of fundamental groups and homology over $\mathbf{Z} \pi, \pi=\pi_{1} X$, through dimension $[n / 2]-1$. Let $H$ be the kernel of $h_{*}: H_{k}(M ; \mathbf{Z} \pi) \rightarrow H_{k}(X ; \mathbf{Z} \pi)$, and choose a set of generators of $H$. These may be represented (by a Whitney theorem) by disjointly embedded spheres with trivial normal bundle, connected to the (pre-assigned) base point. Let $U$ be their union. One can arrange $h^{-1} D=U, D$ an $n$-disk in $X^{n}$.

The normal map $(h|\partial U, b| \partial U)$ is $k$-connected, and $(K, \phi, \mu)$, as in the discussion preceding Theorem 5.6, is defined for this normal map. In fact, $(K, \phi, \mu)$ is hyperbolic in two ways. In this case $K=H_{k}(\partial U ; \mathbf{Z} \pi)$ and the kernels, $L$ and $L^{\prime}$, respectively, of the maps $H_{k}(\partial U ; \mathbf{Z} \pi) \rightarrow H_{k}(U ; \mathbf{Z} \pi)$ and $H_{k}(\partial U ; \mathbf{Z} \pi) \rightarrow H_{k}\left(M_{0} ; \mathbf{Z} \pi\right), M_{0}=$ closure of $M-U$, are subkernels [Wa 3, §5.7]. Poincaré duality shows that they are stably based summands of a suitable type, and a general position and a counting (over $\mathbf{Z} \pi$ ) argument shows that $\phi$ and $\mu$ vanish on bounding classes, i.e. on $L$ and $L^{\prime}$. Further, it is easy to check that if $L$ and $L^{\prime}$ are dual, i.e. $K=L \oplus L^{\prime}$ as stably based modules, then $h$ is a simple homotopy equivalence (see [Wa 3, p. 56]). Also, it is shown in [Wa 3] that there is an automorphism of $(K, \phi, \mu)$ carrying $L$ to $L^{\prime}$, at least after stabilizing so that $L$ and $L^{\prime}$ are actually based.

Hence one may assemble the information as follows: Let $\kappa_{r}$ be the "standard $(-1)^{k}$ hyperbolic form" of dimension $2 r$ (= "standard kernel" of [Wa 3]), with "standard subkernel" $L_{r}$ and with compatible natural identifications $\kappa_{r+1}=\kappa_{r} \oplus \kappa_{1}$. Thus, for some $r$, we have an isomorphism $\rho,(K, \phi, \mu, L) \cong$ $\left(\kappa_{r}, L_{r}\right)$. Let $S U_{r}^{\varepsilon}(\mathbf{Z} \pi)$ be the automorphisms of $\kappa_{r}$ (preserving preferred basis class, of course), $\varepsilon=(-1)^{k}$. Then $S U_{r}^{\varepsilon}\left(\mathbf{Z}_{\pi}\right) \subset S U_{r+1}^{\varepsilon}\left(\mathbf{Z}_{\pi}\right)$ by adding $\mathrm{id}_{\kappa_{1}}$. Let $S U^{e}(\mathbf{Z} \pi)$ be the limit (i.e. the stabilization). The construction of the preceding paragraph together with $\rho$ leads to an element $\alpha \in S U_{r}^{\ell}(\mathbf{Z} \pi) \subset S U^{\ell}(\mathbf{Z} \pi)$.

The choices made, e.g. generators of $H$, possible automorphisms throwing $L$ to $L^{\prime}$, dictate dividing out $S u^{\varepsilon}(\mathrm{Z} \pi)$ by the subgroup $T U^{e}(\mathrm{Z} \pi)$ of stabilizations of elements that preserve $L_{r}$ (as a stably based module). Further, if we choose the generators of $H$ so that the first one, say, is represented by an embedded framed sphere on which it is proposed to perform surgery, then one sees that the effect of this surgery is to replace $\alpha$ by $\sigma \alpha$. Here $\sigma$ is the 
element of $S U^{\varepsilon}(\mathrm{Z} \pi)$ that is the stabilization of the element of $S U_{1}^{\varepsilon}(\mathbf{Z} \pi)$ with matrix $\left(\begin{array}{ll}0 & 1 \\ e\end{array}\right)$ with respect to the "standard base" of $\kappa_{1}$; i.e., $\sigma$ interchanges $L_{1}$ and its dual.

Wall [Wa 3, \$6] proves that the subgroup generated by $T U^{e}(\mathbf{Z} \pi)$ and $\sigma$ is normal, with abelian quotient. We define this quotient to be $L_{n}^{s}(\pi, w)$. Then a rigorous version of the above discussion yields (Theorem 5.6) for $n$ odd also.

To analyze the "kernel" of $\eta$, one uses a geometric realization theorem for elements of the Wall groups; for $\pi=\{e\}$ this is the "plumbing" of Kervaire and Milnor (see [Br 1]), and, in general, a result of Wall [Wa 3, \$5.6].

Theorem 5.7 (Plumbing Theorem). Let $h: M^{n} \rightarrow X$ be a simple homotopy equivalence of compact closed $n$-manifolds, $n>5$. Let $\gamma \in L_{n+1}(\pi, w), \pi=$ $\pi_{1} X$ with $w$ the orientation character of $X$ (first Stiefel-Whitney class). Then there exists a normal cobordism $(f, b)$ of $h$ to a homotopy equivalence $h^{\prime}$ : $M^{\prime} \rightarrow X$, with $\sigma(f, b)=\gamma$.

Note that if $\gamma=0$, then Theorem 5.6 and the $s$-cobordism theorem imply that for any $(f, b)$ as in Theorem 5.7 with $\sigma(f, b)=0$, we have that $h$ and $h^{\prime}$ represent the same element in $\delta_{H}(X)$. So if in the notation of Theorem 5.7, we put $\gamma \cdot[h]=\left[h^{\prime}\right]$, this gives a well-defined action of the group $L_{n+1}^{s}(\pi, w)$ on $\mathcal{S}_{H}(X)$.

Corollary 5.8. The sets of $\eta^{-1}(y)$, for $y \in[X, G / H]$ are precisely the orbits of the action of $L_{n+1}^{s}(\pi, w)$.

We mention that all types of surgery groups are periodic, i.e., $L_{n}^{x}=L_{n+4}^{x}$, for all possible $x$. Further, if $(f, b)$ is a normal map and $\mathbf{C} P^{2}$ is a complex projective 2-space (a 4-dimensional real manifold) then $\sigma(f, b)=\sigma((f, b) \times$ $\mathbf{C} P^{2}$ ).

We may sum up this section in the following theorem.

THEOREM 5.9. There exists a functor $L=\bigoplus L_{m}$ from finitely-presented groups and orientation characters into graded abelian groups such that if $X$ is a (simple) Poincaré complex of dimension $m \geqslant 5$, then there is an exact sequence of pointed sets

$$
L_{m+1}^{s}(\pi, w) \stackrel{\omega}{\rightarrow} \mathcal{S}_{m}^{H}(X) \stackrel{\eta}{\rightarrow} \mathscr{T}_{m}^{H}(X) \stackrel{\sigma}{\rightarrow} L_{m}(\pi, w)
$$

(where $\pi=\pi_{1}(x)$ and $w$ is the orientation character).

In addition, for any $m$, there is a natural isomorphism $L_{m}(\pi, w) \rightarrow$ $L_{m+4}(\pi, w)$, such that the diagram

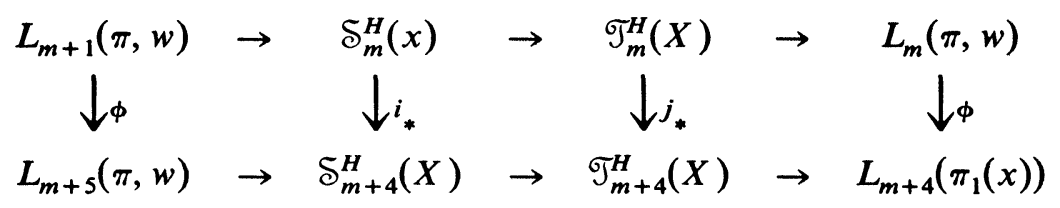

commutes, where $i_{*}(f)$ (resp., $\left.j_{*}(f, b)\right)$ is the normal map induced by $f \times 1$ : $\boldsymbol{M} \times \mathbf{C} P^{2} \rightarrow X \times \mathbf{C} P^{2}$ for $f \in \mathcal{S}_{m}^{H}(X)\left(\right.$ resp., $\left.(f, b) \in \mathscr{T}_{m}^{H}(X)\right)$.

Notes. I. The above theorem is true in the relative case where $(X, Y)$ is a Poincare pair in the sense of [Wa 3] if we either assume $m>6$ or that $Y$ is a topological manifold. 
II. The function $\sigma$ can in fact be defined for any value of $m$. This is not however true for $\omega$. If $m=4$ we can define $\omega$ as well and the sequence (*) remains semiexact. It is, however, not known to be exact. We shall return to this point later.

III. If $\pi_{1}(X)=1$ we have

$$
L_{m}\{1\}= \begin{cases}0 & m \text { odd } \\ Z & m=4 k \\ Z / 2 Z & m=4 k+2\end{cases}
$$

and

$$
\sigma(f, b)= \begin{cases}\frac{1}{8}(\sigma(X)-\sigma(M)) & \text { if } m=4 k \\ \operatorname{Arf} \psi & \text { where } \psi \text { is as in }[\operatorname{Br} 1],[\text { KM 2] }\end{cases}
$$

We thus obtain

Corollary 5.10. Let $(f, b)$ be a normal map, $f\left(M^{m}, \partial M\right) \rightarrow(X, Y)$ with $m \geqslant 5$ and $\pi_{1}(X)=1$ such that $f \mid \partial M$ is an isomorphism on homology.

Then

(1) if $m$ is odd, $(f, b)$ is normally cobordant to a homotopy equivalence,

(2) if $m=4 k,(f, b)$ is normally cobordant to a homotopy equivalence if ana only if $\sigma(M)=\sigma(X)$,

(3) if $m=4 k+2,(f, b)$ is normally cobordant to a homotopy equivalence if and only if $\operatorname{Arf} \psi=0, \psi$ as in $[\mathrm{Br} 1]$.

IV. A theory analogous to the above exists for describing the obstruction to making a normal map by surgery into a (not necessarily simple) homotopy equivalence. The obstruction groups are usually denoted $L_{n}^{h}(\pi, w)$. They are constructed in a way similar to the groups $L_{n}^{s}(\pi, w)$, but without the introduction of stable bases and the demand, at certain points, that certain automorphisms preserve a class of bases. The groups $L_{n}^{h}(\pi, w)$ are related to $L_{n}^{s}(\pi, w)$ by the exact sequence of [Sh 3, \$4.1] and in particular are isomorphic modulo elements of order two.

V. Since $G / H$ is a commutative $H$-space, $[X, G / H]$ and thus $\mathscr{T}^{H}(X)$ have a natural abelian group structure. Nevertheless $\sigma$ is in general not a group homomorphism. However if $X^{m}$ is a manifold then it can be shown that $\sigma$ : $\sigma_{m+1}^{H}(\Sigma(X / \partial X)) \rightarrow L_{m+1}\left(\pi_{1}(X), W\right)$ is in fact a group isomorphism ( $m$ as above). This proves useful in

Theorem 5.11 (WALL) [Wa 3, Chapter 10]. Let $X^{m}$ be a manifold with $m>4\left(m>5\right.$ in the relative case) and $\pi=\pi_{1}(X)$. Then there is an exact sequence

$$
\begin{gathered}
\mathcal{S}_{m+1}^{H}(X \times I, \partial(X \times I)) \stackrel{\eta}{\rightarrow} \mathscr{\sigma}_{m+1}^{H}(X \times I, \partial(X \times I)) \\
\stackrel{\theta}{\rightarrow} L_{m+1}(\pi, w) \stackrel{\omega}{\rightarrow} \mathcal{S}_{m}^{H}(X, \partial X)
\end{gathered}
$$

with $\theta$ a group homomorphism. (Note that $I=[0,1]$, and we again point out that by $\mathscr{T}(X \times I, \partial(X \times I)) \approx \mathcal{T}(\Sigma(X / \partial X))$ in this context we mean the normal cobordism classes of degree 1 normal map $(f, b):(M, \partial M) \rightarrow(X \times$ $I, \partial(X \times I))$ with $f \mid \partial M$ an isomorphism (rather than just a homotopy equivalence) and the normal cobordism being a product on boundaries.) 
5.2 Surgery and the smoothing and triangulations of manifolds. Having briefly sketched the framework of surgery theory we now describe some of its applications. We again point out that the theorems of $\$ 5.1$, dependent as they are on the Whitney trick, are known to be true only under the dimensional restrictions (usually $m \geqslant 5$ or $m \geqslant 6$ ) stated in their hypotheses. Thus although they do not tell us what is happening in 4-dimensional topology they do indicate what types of results to look for.

The most striking applications of surgery theory is to the classification of manifold structures. Thus let $\left(H_{2}, H_{1}\right)$ denote any of the pairs $(\mathrm{PL}, \mathrm{O})$ or (TOP, O) or (TOP, PL) (or the corresponding nonstable pairs). Clearly if $X$ is an $H_{1}$-manifold it has the underlying structure of an $H_{2}$-manifold as well. Now suppose that $X$ is an $H_{2}$-manifold. Then giving an $H_{1}$-structure $\Sigma$ on $X$ is equivalent to asserting the existence of an $H_{1}$-manifold $M$ and an $H_{2}$-homeomorphism $f: M \rightarrow X$.

One can impose three distinct equivalence relations on such $H_{1}$-structures.

1. CONCORDANCE. Our previous definition (see the introduction) translates as follows: $f_{i}: M_{i} \rightarrow X, i=0,1$, are concordant if and only if there exist an $H_{1}$-manifold $N$ and an $H_{2}$-homeomorphism $F: N \rightarrow X \times I$ with $F \mid F^{-1}(X \times$ $\{i\})=f_{i}$.

2. ISOMORPHISM. There exists an $H_{1}$-isomorphism $\phi: M_{1} \rightarrow M_{2}$ making

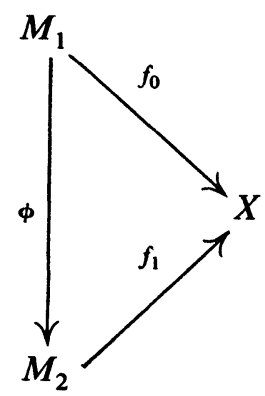

commute.

3. IsoTopy. There exists an $H_{1}$-isomorphism, $\phi: M_{1} \rightarrow M_{2}$, isotopic to the identity and making

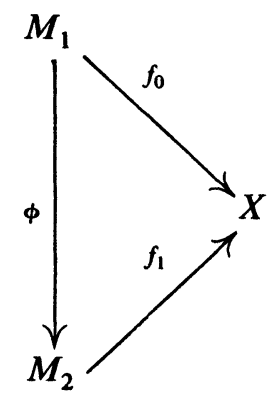

commute.

Clearly Isotopy $\Rightarrow$ Isomorphism but Isomorphism $\nRightarrow$ Isotopy. (In fact there exist nonisotopic smooth structures on $S^{7}$ which are isomorphic. See [KS 1, Essay 1].) 
We now define

$$
\begin{aligned}
S_{H_{2} / H_{1}}(X) & =\text { concordance classes of } H_{1} \text {-structures on } H_{2}, \\
\mathscr{G}_{H_{2} / H_{1}}(X) & =\text { isotopy classes of } H_{1} \text {-structures on } H_{2}, \\
\text { Isom }_{H_{2} / H_{1}}(X) & =\text { isomorphism classes of } H_{1} \text {-structures on } H_{2} .
\end{aligned}
$$

Lastly recalling our discussion in previous sections we note that one can define analogues which are adequate substitutes for tangent bundles in all our categories $H$ above and such bundles are classified by maps $M \rightarrow B_{H(k)}$ (or $M \rightarrow B_{H}$ in the stable case).

Notice that tangent bundles in a given category $H$ are $H$-manifolds. (There are certain technical problems in PL. Again see [KS 1] for further explanations.)

If $X$ is an $H_{1}$-manifold, its tangent bundle has an $H_{1}$-structure. By 'forgetfullness' it then has the structure of an $H_{2}$-bundle as well. [Again there occur some difficulties in going from DIFF to PL which we suppress.]

Now conversely suppose $X$ has an $H_{2}$-structure and let $\mathcal{T}: X \rightarrow B_{H_{2}}$ be its (stable) tangent bundle. Then by a tangential $H_{1}$-structure on $X$ we will mean a lift $\mathcal{T}^{\prime}$ of $\mathcal{T}: X \rightarrow B_{H_{2}}$ to a commutative diagram

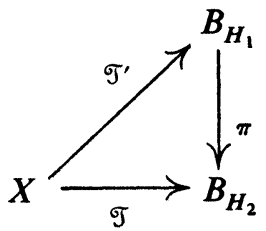

We call two lifts $\mathcal{T}, \mathcal{T}^{\prime}$ equivalent if they are fiber homotopic.

Then as in our discussion of $[X, G / H]$ we have that equivalence classes of tangential $H_{1}$-structures correspond to homotopy classes of cross sections of the bundle $\mathcal{T}^{*}[\pi]$ with fiber $H_{2} / H_{1}$ induced by $\pi$. We will denote the equivalence classes of stable tangential $H_{1}$-structures on $X$ by $\sigma_{H_{2} / H_{1}}(X)$. Since the fibration $B_{H_{1}} \rightarrow B_{H_{2}}$ acts like a principal fibration we see that if $\widetilde{T}_{H_{2} / H_{1}}(X) \neq \varnothing$ then $\mathcal{T}_{H_{2} / H_{1}}(X)=\left[X, H_{2} / H_{1}\right]$.

Clearly if an $H_{2}$-manifold $X$ is to have an $H_{1}$-structure its stable tangent bundle must have an $H_{1}$-structure. Thus a necessary condition for the existence of an $H_{1}$-structure on $X$ is that $\mathscr{T}_{H_{2} / H_{1}}(X)$ not vanish or equivalently that the bundle $\sigma^{*}[\pi]$ have a cross section. Using obstruction theory one sees that the obstruction to the existence of such cross sections lies in groups of the form $H^{i}\left(X ; \pi_{i-1}\left(H_{2} / H_{1}\right)\right)$ while obstructions to homotoping one cross section to another lies in $H^{i}\left(X, \pi_{i}\left(H_{2} / H_{1}\right)\right)$.

For future use we recall the following deep results. (See [KS 1] for details and reference.) (We note that the following are difficult calculations involving among other things the use of Rohlin's theorem, nonsimply connected surgery, the $s$-cobordism theorem and deep results of Cerf [Cerf 1], [Cerf 2].)

(1) Corresponding to $B_{H_{1}} \rightarrow B_{H_{2}} \rightarrow B_{G}$ with $\left(H_{2}, H_{1}\right)=(\mathrm{PL}, \mathrm{O})$ or (TOP, O) or (TOP, PL) we have 'principal' fibrations $H_{2} / H_{1} \rightarrow G / H_{1} \rightarrow$ $G / H_{2}$ and similarly with $\left(G, H_{2}, H_{1}\right)$ replaced by $\left(G(k), H_{2}(k), H_{1}(k)\right)$ or by (TOP, PL, O), (TOP $(k), \operatorname{PL}(k), \mathrm{O}(k))$. 
(2) $\mathrm{TOP}(k) / \mathrm{PL}(k) \simeq \mathrm{TOP} / \mathrm{PL} \simeq K\left(\mathrm{Z}_{2}, 3\right)$ for $k>5$. $\mathrm{TOP}(k) / \mathrm{PL}(k)$ is contractible for $k \leqslant 3$.

(3) (i)

$$
\pi_{m}(\mathrm{TOP} / \mathrm{O})=\left\{\begin{array}{l}
\Theta_{m}, \quad m \geqslant 5, \\
\pi_{m} K\left(\mathrm{Z}_{2}, 3\right), \quad m \leqslant 6,
\end{array}\right.
$$

where $\Theta_{m}$ is the group of $h$-cobordism classes of smooth homotopy $n$-spheres. (See [KM 2] for some calculations.)

(ii) $\operatorname{TOP}(k) / \mathrm{O}(k)$ is contractible for $k=2$ and

$$
\begin{aligned}
& \pi_{i}(\mathrm{TOP}(3) / \mathrm{O}(3))=0 \text { for } i \leqslant 4 \text {, } \\
& \pi_{k}(\mathrm{TOP} / \mathrm{O}, \mathrm{TOP}(m) / \mathrm{O}(m))=0 \quad k \leqslant m+2 \text { and } m \geqslant 5 \text {, } \\
& \pi_{m}(G / \mathrm{PL})=\pi_{m}(G / \mathrm{TOP})=\left\{\begin{array}{lll}
0, & m \equiv 1,3 & (\bmod 4), \\
\mathbf{Z}_{2}, & m \equiv 2 & (\bmod 4), \\
\mathbf{Z}, & m \equiv 0 & (\bmod 4),
\end{array}\right.
\end{aligned}
$$

with the natural fibration $B_{\mathrm{PL}} \rightarrow B_{\text {TOP }} \rightarrow B_{G}$ inducing $\pi_{i}(G / \mathrm{PL}) \approx$ $\pi_{i}(G / \mathrm{TOP})$ for $i \neq 4$ and the commutative diagram

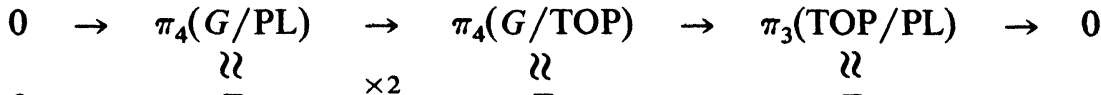

$$
\begin{aligned}
& \begin{array}{llllllll}
0 & \rightarrow & \mathrm{Z} & \stackrel{\times 2}{\rightarrow} & \mathrm{Z} & \rightarrow & \mathrm{Z}_{2} & \rightarrow
\end{array}
\end{aligned}
$$

otherwise.

$$
\pi_{i}(\mathrm{PL} / \mathrm{O}) \approx \begin{cases}\pi_{i}(\mathrm{TOP} / \mathrm{O}) & i>4, \\ 0 & i<6 .\end{cases}
$$

Since $\mathrm{TOP} / \mathrm{PL} \approx K\left(\mathrm{Z}_{2}, 3\right)$ we immediately obtain the following classification result.

LEMMA 5.12. Let $X$ be a topological manifold.

(1) Then $X$ has a stable tangential PL-structure if $H^{4}\left(X ; \mathbf{Z}_{2}\right)=0$.

(2) Given a stable tangential PL-structure on $X$ then the number of distinct stable tangential PL-structures on $X$ is in 1-1 correspondence with $H^{3}\left(X ; \mathbf{Z}_{2}\right)$.

To obtain a result giving us information about the relation between the stable tangential $H_{1}$-structures on the $H_{2}$-manifold $X$ and actual $H_{1}$-structures on $X$ we must relate $\mathcal{T}_{H_{2} / H_{1}}(X)$ with $\oint_{H_{2} / H_{1}}(X)$ or $\operatorname{Isom}_{H_{2} / H_{1}}(X)$.

Thus as a first step let $\eta: \mathcal{S}_{H_{2} / H_{1}}(X) \rightarrow{ }_{H_{2} / H_{1}}(X)$ be the obvious map associating the $H_{1}$-structure of its stable tangent bundle to any $H_{1}$-structure on the $H_{2}$-manifold $X$. Ideally we would show that $\eta$ is a bijective correspondence. This however is not always true! (It is false for 3-manifolds and $\left(H_{2}, H_{1}\right)=(T O P, P L)$ as a consequence of results of Moise [Moise].)

What can be shown is

Theorem 5.13 [KS 1], [KS 2] (Stable Classification theOReM). Let $\left(\mathrm{H}_{2}, \mathrm{H}_{1}\right)$ be either (TOP, PL), (PL, DIFF) or (TOP, DIFF).

Let $\mathrm{M}^{n}$ be an $\mathrm{H}_{2}$-manifold. 


\section{Then}

(1) if $\left(H_{2}, H_{1}\right)=(\mathrm{PL}, \mathrm{DIFF})$ then $\eta: \mathfrak{S}_{H_{2} / H_{1}}(M) \rightarrow \mathcal{T}_{H_{2} / H_{1}}(M)$ is a bijection, and

(2) if $\left(H_{2}, H_{1}\right)=($ TOP, PL) or (TOP, DIFF) and $n \neq 3,4$ then $\eta$ is a bijection.

If $\left(H_{2}, H_{1}\right)=$ (TOP, PL) then Theorem 5.13 fails for 3-manifolds.

Theorem 5.13 is essentially based on two ingredients.

Let $\mathcal{T}_{H_{2}(n) / H_{1}(n)}\left(X^{m}\right), n \geqslant m$, denote the equivalence class of lifts of $\mathcal{T}_{\text {: }}$ $X \rightarrow B_{H_{2}(n)}$ to $\mathcal{F}^{\prime}: X \rightarrow B_{H_{1}(n)}$. That is $\mathcal{T}_{H_{2}(n) / H_{1}(n)}$ are the $H_{1}$-structures on $\mathscr{T}_{X} \oplus \varepsilon^{n-m}\left(\varepsilon^{n-m}\right.$ is the $n-m$ plane trivial bundle over $X$ ). (If $\mathscr{T}_{H_{2}(n) / H_{1}(n)}\left(X^{m}\right) \neq 0$ then it equals $\left[X^{m}, H_{2}(n) / H_{1}(n)\right]$.)

The first ingredient needed in the proof of Theorem 5.13 is then:

TheOREM 5.13'. Let $n \geqslant 1$ and let $\left(H_{2}, H_{1}\right)$ be as in Theorem 5.13. Let $\left(H_{2}(n), H_{1}(n)\right)$ be either $(\operatorname{PL}(n), \operatorname{DIFF}(n))$ or $(\mathrm{TOP}(n), \operatorname{DIFF}(n))$ or $(\mathrm{TOP}(n)$, $\operatorname{PL}(n))$.

Suppose $\mathrm{M}^{n}$ is an $\mathrm{H}_{2}$-manifold.

Then

(1) if $\left(H_{2}, H_{1}\right)=(\mathrm{PL}, \mathrm{DIFF})$ then $\eta: \delta_{\mathrm{PL} / \mathrm{DIFF}}(M) \rightarrow \mathscr{T}_{\mathrm{PL}(n) / \operatorname{DIFF}(n)}(M)$ is $a$ bijection;

(2) if $H_{2}=$ TOP and $H=H_{1}=$ DIFF or PL then, if $n \neq 4, \eta: \delta_{\mathrm{TOP} / H}(M)$ $\rightarrow \widetilde{T}_{\mathrm{TOP}(n) / H(n)}(M)$ is a bijection.

(Note that Theorem $5.13^{\prime}$ is true for (TOP, $H$ ) if $n=3$ even though Theorem 5.13 is not.)

What Theorem $5.13^{\prime}$ is asserting is that if the tangent bundle $\tau$ of an $H_{2}$-manifold $M$ has an $H_{1}$-structure then $M$ itself has an $H_{1}$-structure inducing that of $\mathcal{T}$. Its proof if $n \neq 3$ can be reduced to the following product-structure theorem.

Theorem 5.14 [Product STRUCTURe] [KS 1], [KS 2]. Let $\left(H_{2}, H_{1}\right)$ be as above. Let $M^{n}$ be an $H_{2}$-manifold. Suppose $\Sigma$ is an $H_{1}$-structure on $M^{n} \times R^{s}$, $s \geqslant 1$. If either $\left(H_{2}, H_{1}\right)=(\mathrm{PL}, \mathrm{DIFF})$ or $\left(H_{2}, H_{1}\right)=(\mathrm{TOP}, \mathrm{DIFF})$ or (TOP, PL) and $n \neq 3,4$, then there exists an $H_{1}$-structure $\theta$ on $M^{n}$ such that $M_{\theta} \times R^{s}$ is concordant to $\left(M \times R^{s}\right)_{\Sigma}$. Furthermore $\theta$ is unique up to concordance.

[Theorem 5.14 fails if $n=3$ and $\left(H_{2}, H_{1}\right)=$ (TOP, PL) say. In particular if $M=S^{3}$ then $S^{3} \times R^{s}$ for $s \geqslant 2$ has two distinct PL-structures while by Moise's theorem $S^{3}$ has a unique PL-structure.]

Then Theorem 5.13 is deduced by showing that under the hypothesis of Theorem 5.13, stable classification is implied by nonstable classification which in turn can be deduced from Theorem 5.14. That is one has:

THEOREM 5.15. Under the hypothesis of Theorem 5.13 there is a bijective correspondence between $\mathscr{T}_{H_{2}(n) / H_{1}(n)}\left(M^{n}\right)$ and $\mathcal{T}_{H_{2} / H_{1}}\left(M^{n}\right)$. (Note that $\sigma_{\mathrm{TOP}(3) / \mathrm{PL}(3)}\left(S^{3}\right)=\{e\}$ while $\operatorname{G}_{\mathrm{TOP} / \mathrm{PL}}\left(S^{3}\right) \approx H^{3}\left(S^{3} \mathbf{Z}_{2}\right)=\mathbf{Z}_{2}$ since $\mathrm{TOP}(q) / \mathrm{PL}(q)$ is contractible for $q \leqslant 3$ while TOP $/ \mathrm{PL} \approx K\left(\mathbf{Z}_{2}, 3\right)$.) 
Lastly one must make the transition from concordance classes to isomorphism classes. This unfortunately can not be done. However we can establish in the appropriate dimensions a bijective correspondence between isotopy classes and concordance classes.

THEOREM 5.16 [CONCORDANCE IMPLIES ISOTOPY]. Under the same conditions as above there is a bijective correspondence between $\oint_{H_{2} / H_{1}}(X)$ and $\mathcal{S}_{H_{2} / H_{1}}(X)$.

Before continuing, we mention what ingredients are required to prove Theorems 5.14 and 5.15. We must distinguish the case $\left(H_{2}, H_{1}\right)=$ (PL, DIFF) from the other cases.

If $\left(H_{2}, H_{1}\right)=(\mathrm{PL}, \mathrm{DIFF})$ the methods involved are the smoothing methods developed by Cairns, Munkres, Hirsch and Mazur ([Ca 1], [LR 2], [Mun 1], [Mun 2], [Hi 1], [Hi 2], [HM]). They do not depend on either surgery theory or the $h$-or $s$-cobordism theorem in any way. In fact the smoothability of all PL-manifolds of dimension $\leqslant 4$ was already demonstrated in 1944 in [Ca 2], [Ca 3]!

Now suppose $H_{2}=$ TOP and $H_{1}=$ PL or DIFF. If $n \leqslant 2$ then all our results essentially reduce to classical results about the existence and uniqueness of triangulations of surfaces $F^{2}$. ( $n=1$ is of course completely trivial.) For $n=3$ this same result is due to Moise [Moise] by entirely different methods than those used in the higher-dimensional case. If $n \geqslant 5$ then the above theorems are due to Kirby and Siebenmann [KS 1], [KS 2]. See also Lashof and Rothenberg [LR]. The proof of Theorem 5.15 is based on the use of the handle straightening machinery developed in [KS 1]. This in turn involves heavy use of the PL and DIFF $s$-cobordism theorem. Theorem 5.14 is in turn equivalent to Theorem 5.16 plus the stable homeomorphism theorem of Kirby [Kirb 4], which is based on heavy use of nonsimply connected surgery.

(A homeomorphism $h: R^{n} \rightarrow \mathbf{R}^{n}$ is called stable if it can be written as a finite composition of homeomorphisms each of which is somewhere the identity. The stable homeomorphism conjecture is then:

$\mathrm{SHC}_{n}$ : All orientation preserving homeomorphisms of $\mathbf{R}^{n}$ are stable. This is closely related to the annulus conjecture:

$\mathrm{AC}_{n}$ : Let $f, g: S^{n-1} \rightarrow R^{n}$ be disjoint, locally flat imbeddings with $f\left(S^{n-1}\right)$ inside the bounded component of $\mathbf{R}^{n}-g\left(S^{n-1}\right)$. Then the closed region $A$ bounded by $f\left(S^{n-1}\right)$ and $g\left(S^{n-1}\right)$ is homeomorphic to $S^{n-1} \times[0,1]$.

In fact $\mathrm{SHC}_{n} \Rightarrow \mathrm{AC}_{n}$ and $\mathrm{AC}_{k}$ for all $k \leqslant n$ implies $\mathrm{SHC}_{n}$. (It is easy to show ([RS], [RIf]) that the open region between $f\left(S^{n-1}\right)$ and $g\left(S^{n-1}\right)$ is always homeomorphic to $S^{n-1} \times(0,1)$.)

Kirby showed in [Kirb 4], using a new idea of furling and unfurling tori as well as the homotopy classification of tori due to [Wa 5] and [HS 1], [HS 2] (which is based on the full power of nonsimply connected surgery) that $\mathrm{SHC}_{n}$ is true for all $n \geqslant 5$. (It was classically known to be true for $n \leqslant 3$ ).)

We note that the truth of Theorem 5.16 in dimension 4 would imply $\mathrm{SHC}_{4}$ and thus in dimension 4 Theorem 5.16 is equivalent to Theorem 5.14.

As neither the stable homeomorphism theorem nor the $s$-cobordism theorem is known for dimension 4 the proofs of 5.14 and 5.15 clearly break down 
in this dimension. Furthermore as we pointed out Theorem 5.14 is false if dimension $(M)=3$. This in turn implies that Theorem 5.15 must fail in either dimension 3 or 4 since its truth in all dimensions would imply (see [Kirb 3]) that the product structure theorem is true in all dimensions. Lastly since the product structure theorem fails in dimension 4, essentially nothing is known about TOP(4)/H(4), $H=$ PL or DIFF and there is thus no analogue of Theorem 5.15 in dimension 4.

Putting together our theorems above we arrive at the following classification theorem.

THEOREM 5.17. Let $\left(\mathrm{H}_{2}, \mathrm{H}_{1}\right)$ be as above and suppose $\mathrm{X}^{q}$ is an $\mathrm{H}_{2}$-manifold. Then

(1) If $\left(H_{2}, H_{1}\right)=(\mathrm{PL}$, DIFF) then $X$ admits a smooth structure if and only if a certain PL/DIFF bundle over $X$ has a cross section. If $X$ admits a smooth structure then isotopy classes of smooth structures are in 1-1 correspondence with [ $X, \mathrm{PL} / \mathrm{DIFF}]$.

Suppose $q \neq 3,4$.

Then:

(2) If $H_{2}=$ TOP and $H=H_{1}=$ DIFF or PL then $X$ admits an $H$-structure if and only if a certain TOP $/ H$ bundle over $X$ has a cross section. If $H=$ PL then equivalently $X$ admits $a$ PL-structure if and only if a certain obstruction $\sigma(X) \in H^{4}\left(X ; \mathbf{Z}_{2}\right)$ vanishes.

If $X$ admits an $H$-structure then isotopy classes of $H$-structures are in 1-1 correspondence with $[X, \mathrm{TOP} / H]$.

If $H=\mathrm{PL}$ this in turn is in 1-1 correspondence with $H^{3}\left(X ; \mathbf{Z}_{2}\right)$.

Using the product structure theorem one can also deduce the following theorems.

THEOREM 5.18 (TOPOLOGICAL TRANSVERSALITY). If $f: M^{n} \rightarrow Y^{n+q}$ is a continuous function between TOP manifolds and $X^{q}$ is a locally flat TOP submanifold of $Y$. Then if $m \neq 4 \neq n-m, f$ is homotopic to a map $f^{\prime}$ which is transverse regular to $X^{q}$.

THEOREM 5.19. Every TOP manifold $X^{n}$ with $n \geqslant 6$ has a topological handlebody-decomposition.

5.3 Triangulating 4-manifolds and some consequences of Rohlin's theorem. We now turn to dimension 4 manifolds. The classification theorem above combined with the fact that $\pi_{i}(\mathrm{PL} / \mathrm{DIFF})=0$ for $i<7$ shows that every PL 4-manifold has a unique differentiable structure. This is the result of Cairns [Ca 2], [Ca 3] already mentioned.

As we already mentioned the (TOP, $H$ ) product structure theorem is false in dimension 3. Its status in dimension 4 is as yet open. If it is true in dimension 4 then Theorems 5.13, 5.14, 5.15, 5.16, 5.17, 5.18 and 5.19 are also true in that dimension! and Theorem 5.13' is then in turn true in all dimensions! [Kirb 4]. In particular one also has that TOP(4)/PL(4) $\simeq$ $\mathrm{TOP} / \mathrm{PL} \simeq K\left(\mathrm{Z}_{2}, 3\right)$. (We note that if $M^{4}$ is a compact topological 4-manifold whose tangent bundle $\tau$ admits a PL structure then so does $M-\{\mathrm{pt}\}$. 
Furthermore if $\alpha, \beta$ are stably equivalent PL structure on $M$ then they are concordant. See [LR], [LR 2].) In addition $\mathrm{SHC}_{4}$ and thus $\mathrm{AC}_{4}$ would be true!

As we already pointed out product structure for $n=4$ is equivalent to concordance implies isotopy for $n=4$ and implies topological transversality for $n=4$. In [Kirb 4], Kirby makes the attractive conjecture that:

CONJECTURE (Kirby). The product structure theorem in dimension 4 is true.

We note that four-dimensional topological transversality in turn implies that there exists a compact simply-connected unbounded topological manifold $M$ which is almost parallelizable and has $\sigma(M)=8$. (Proof: By Edwards [Ed 1] $c P \times S^{1}, P$ the Poincaré homology sphere, is a topological manifold. Making the projection map $\pi: c P \times S^{1} \rightarrow S^{1}$ transverse regular to $* \in S^{1}$ gives the desired 4-manifold $M=\pi^{-1}(*)$.) Note that $M$ of course is not combinatorially triangulable!

Scharlemann [Sch 3] in turn has shown that if such a topological manifold exists then Theorem 5.18 holds without any restriction. (The proof of this runs as follows: Suppose $M$ as above exists. Then $M-\{\mathrm{pt}\}$ is an open parallelizable manifold and so by [Lash] is smoothable. Then the induced smoothing on a neighborhood of the excluded point must be exotic for otherwise $M$ would itself be smoothable. There therefore exists an exotic smoothing $\Omega$ (i.e. not concordant to the usual one) of $\left(S^{3} \times R\right)$. One then notes that one still has DIFF transversality in $\left(S^{3} \times R\right)_{\Omega}$ (i.e. $h: \mathbf{R}^{4} \times R^{1} \rightarrow$ $R^{1}$ is DIFF transverse to 0 in $\left.\left[\left(R^{4}-0\right) \times R^{1}\right]_{\Omega}\right)$.

Now it is easy to see that TOP transversality holds in general in dimension 4 provided it holds for any continuous map $f: R^{4} \times R^{1} \rightarrow R^{1}$ and $0 \in R^{1}$. One can now construct a map $f^{1}: \mathbf{R}^{4} \times R^{1} \rightarrow R^{1}$ such that $f^{1}=f$ on $\mathbf{R}^{4} \times R^{1}-\left(\dot{D}^{4} \times \dot{D}_{1}\right)$ and $f^{1}(x, y)=h(2 x, 2 y)$ ( $h$ as above) on $\left(\frac{1}{2} B^{4}\right.$ $\left.\times \frac{1}{2} B^{1}\right)$ and $f^{1}$ extends nicely on $B^{4} \times B^{1}-\left(\frac{1}{2} B^{4} \times \frac{1}{2} B^{1}\right)$. Using the DIFF transversality of $h$ we conclude that $f^{1}$ is DIFF transverse to 0 . But clearly $f^{1}$ is homotopic to $f$ and so TOP transversality holds! (See [Sch 3] for further details.)

We have thus seen that four-dimensional topological transversality is equivalent to the failure of Rohlin's theorem for TOP manifolds. As we noted in Chapter 1 Rohlin's theorem points out an anomaly in the behavior of 4-dimensional PL-manifolds. [In all other dimensions congruent to zero (mod 4) there exist closed almost parallelizable PL spin manifolds of signature 8!] This anomaly has been exploited by Siebenmann [Sb 1] to prove that the DIFF (PL) $s$-cobordism theorem must be false in dimension 4 or 5 . In particular we have:

Theorem 5.20 (SiebenmanN). For either $n=4$ or $n=5$ (or both) there exists an s-cobordism from $T^{n}$ to itself which is not a product $\left(T^{n}=n\right.$-torus).

The proof of Theorem 5.20 uses the assumed existence of an s-cobordism theorem in dimensions 4 and 5 to produce (via the Wall [Wa 5], Hsiang and Shaneson [HS 1], [HS 2] classification of fake tori) a compact 4-dimensional PL or DIFF $M$ with $w_{2}(M)=0$ and $\sigma(M)=8$. This however contradicts Rohlin's theorem and so Theorem 5.20 follows.

The results of Hsiang and Shaneson and Wall provide us with one of the 
prettiest applications of Rohlin's theorem and high-dimensional nonsimplyconnected surgery. Essentially they use surgery theory to construct exotic homotopy equivalences $f: W \rightarrow T^{n}$ and then use Rohlin's theorem to show that for certain of these $f, W$ isn't PL-homeomorphic to $T^{n}$. For example if $n=5$ there exist exactly three homotopy tori (two fake and one honest one). Siebenmann [Sb 3] then used these classification results to disprove the Hauptvermuting for manifolds of dimension greater than five. (Recall that the Hauptvermuting is essentially the assertion that PL-structures on TOP manifolds are unique up to isomorphism.) (Note that the classification results of [KS 1], [KS 2] mentioned earlier classify PL-structures up to isotopy and thus can not be used directly to disprove the Hauptvermuting. As we pointed out earlier nonisotopic structures may be isomorphic!).) More precisely [HS 1], [HS 2], [Wa 5] prove that there exists a PL-automorphism $\alpha$ of $B^{2} \times T^{n}$, $n \geqslant 3$, such that (1) the automorphism $\beta$ induced by $\alpha$ on the quotient $T^{2+n}$ of $B^{2} \times T^{n}$ (obtained by identifying opposite sides of the square $B^{2}$ ) has mapping torus

$$
T(\beta)=I \times T^{2+n} /(0, x) \sim(1, \beta(x))
$$

not PL-isomorphic to $T^{3+n}$.

(2) There exists a PL-cobordism $\left(W, T^{n+3}, T(\beta)\right)$ and a homotopy equivalence of $W$ to $\left(\left(I \times T^{3}\right) \# Q\right) \times T^{n}$ extending the standard homotopy equivalences $T^{3+n} \simeq 0 \times T^{3} \times T^{n}$ and $T(\beta) \simeq 1 \times T^{3} \times T^{n}$, where $Q$ is the Poincaré complex $M_{1}^{4}$ discussed in $\$ 1.3$.

Siebenmann then showed that there exists a topological homeomorphism $h$ : $T^{3+n} \approx T(\beta)$ which is not isomorphic to a PL-homeomorphism. (If $h$ were isomorphic to a PL-homeomorphism we could use it to identify $T^{3+n}$ with $T^{\beta}$ in $W$ above. This would give rise to a PL-manifold $X^{4+n} \simeq\left(S^{1} \times T^{3} \# Q\right)$ $\times T^{n}$ and use of PL-transversality would give a 4-manifold $M$ with $w_{1}(M)=$ $w_{2}(M)=0$ and $\sigma(M)=\sigma\left(S^{1} \times T^{3} \# Q\right)=\sigma(Q)=8$, contradicting Rohlin's theorem.) One also obtains [Sb 1]:

THEOREM 5.21. There exists a closed orientable TOP manifold of dimension either 4 or 5 that admits no topological handle decomposition.

PROOF. If Rohlin's theorem were known to be false in TOP then any counterexample $M^{4}$ would not have a handle decomposition. (Proof: Using Moise's results on the existence and uniqueness of 3-dimensional triangulations one can show that any 4-manifold which has a handlebody decomposition admits a PL-structure.) Not having such a counterexample one proceeds as follows.

Let $W^{5}=Q \times S^{1}$ ( $Q$ as above) and recall that by [Ed 1] $W^{5}$ is a topological manifold. We assert that if $\bar{W}^{5}$, the infinite cyclic cover of $W^{5}$, has a handle decomposition then Rohlin's theorem fails for TOP.

Proof. The idea here is to pick a compactum $K$ in $\bar{W}^{5}$ such that $\bar{W}^{5}-K$ has two unbounded components. Let $H$ be a finite handlebody containing $K$ so that $H$ separates the ends of $\bar{W}^{5}$. Thus $\partial H$ separates the ends of $\bar{W}^{5}$. One can then check using homology theory that some unbounded compact connected component $X$ of $\partial H$ separates the ends of $\bar{W}^{5}$. One easily checks that $w_{2}\left(W^{5}\right)=0$ and since $X$ has a trivial normal bundle in $W^{5}$ it must have 
$w_{2}(X)=0$. One can then show that $\bar{W}^{5} \times \mathbf{C} P^{2}=M_{1}^{8} \times R$ (where $M_{1}^{8}$ is the manifold we constructed in Chapter $1, \S 3$ ) and that $M_{1}^{8}$ is cobordant to $X \times \mathbf{C} P^{2}$. Then $X, M_{1}^{8}, X \times \mathbf{C} P^{2}$ must all have the same signature which is 8. Thus Rohlin's theorem fails if $W^{5}$ is handleable. In particular we have proven Theorem 5.21.

(We note that one can prove Theorem 5.21 without using the result of Edwards quoted above. One can in fact establish using surgery theory alone the existence of a topological manifold $\tilde{W}^{5}$ homotopy equivalent to $X^{4} \times S^{1}$ and continue the proof using $\tilde{W}$ wherever we used $W$. This was the method used by Siebenmann to prove Theorem 5.21 some years before Edward's double suspension theorem was proven!)

Recently Siebenmann and Matumoto ([MSb]) have extended 5.20 to the topological category as well. They prove

TheOREM 5.20' (MATumoto AND SiebenmanN). There exists a compact topological ( $=\mathrm{TOP})$ cobordism $\left(W ; V, V^{1}\right)$ that is not a product cobordism, i.e. not homeomorphic to $(V \times I ; V \times 0, V \times 1)$.

Indeed there exists such an $s$-cobordism with either $V=V^{1}=S^{1} \times \mathbf{R} P^{2}$ or $V=V^{1}=T^{2} \times \mathbf{R} P^{2}$ but it is not known which.

The proof of Theorem 5.20 arises from the existence of normal invariants in $\left[I \times \mathbf{R} P^{2} \times S^{1}, G /\right.$ TOP $]$ which do not come from $\left[I \times \mathbf{R} P^{2} \times\right.$ $\left.S^{1}, G / P L\right]$. We refer the reader to [MSb] for details.

Although one has no counterpart of higher-dimensional smoothing and triangulability theorems in dimension 4 one can get some results modulo connected sums with $S^{2} \times S^{2}$ s (i.e. stable results in the sense of Chapter $1, \S 2)$. This should not be too surprising since as we showed in Chapter 1 the $s$-cobordism theorem is true modulo connected sums with $S^{2} \times S^{2}$ in dimension 4.

In particular Cappell, Lashof and Shaneson ([LS], [CLS] see also [Sch 2]) have shown:

THEOREM 5.22 [LS]. Let $M$ be a closed 4-manifold. Then the topological tangent bundle $\mathcal{T}_{M}$ of $M$ lifts to a smooth vector bundle if and only if for some $k \geqslant 0, M \# k\left(S^{2} \times S^{2}\right)$ admits a smooth structure. Furthermore if $M_{\alpha}, M_{\beta}$ are two smoothings of $M$ inducing equivalent lifts of $\mathcal{T}_{M}$ to a smooth vector bundle then for some $k$, the inclusion map $i: M^{0} \rightarrow M \# k\left(S^{2} \times S^{2}\right)$ is isotopic to a smooth embedding of $\left(M^{0}\right)_{\alpha}$ in $M_{\beta} \# k\left(S^{2} \times S^{2}\right)\left[M^{0}\right.$ is $\overline{M-4-d i s c .]}$

We note that since we do not know if $\pi_{i}(\mathrm{TOP}(4) / \mathrm{PL}(4)) \rightarrow \pi_{i}(\mathrm{TOP} / \mathrm{PL})$ is a monomorphism for any $i$, the foregoing result is not usable to obtain any information about smoothings directly from stable liftings of two lifts. By a direct argument one can obtain:

THEOREM 5.23 [LS]. Let $M^{4}$ be a closed topological 4-manifold whose stable tangent bundle lifts to a smooth vector bundle. Then for $k$ sufficiently large, any connected sum $M \# k\left(S^{2} \times S^{2}\right)$ is h-cobordant to a smooth manifold.

The arguments used to prove Theorems 5.22 and 5.23 use the stabilization arguments found in [Wa 1], [Wa 2] [CS 1] to circumvent the difficulties due to the nonexistence of the $s$-cobordism theorem in dimension 4. 
One can similarly establish analogues of the product structure theorem in dimension 4 if one is willing to allow connect summing with $S^{2} \times S^{2}$ s.

More precisely one has by methods similar to the above:

THEOREM 5.24 [CLS]. Suppose $X$ is a closed topological manifold and $\theta$ is a smooth structure on $X \times R$. Then for some $k \geqslant 0,\left[\left(X \# k\left(S^{2} \times S^{2}\right)\right)\right]_{\theta} \times R$ is diffeomorphic to $M \times R, M$ a smooth closed 4-manifold. Furthermore if for some $k^{\prime} \geqslant 0,\left[X \# k^{\prime}\left(S^{2} \times S^{2}\right)\right]_{\theta} \times R$ is diffeomorphic to $M^{\prime} \times R, M^{\prime}$ a smooth closed 4-manifold, then there exist $k_{1}, k_{2} \geqslant 0$ such that $M \# k_{1}\left(S^{2} \times\right.$ $\left.S^{2}\right)$ is diffeomorphic to $M^{\prime} \# k_{2}\left(S^{2} \times S^{2}\right)$.

Using the methods of [CLS] one can also establish

THEOREM 5.25. (1) If $\pi_{3}(\mathrm{TOP}(4) / \mathrm{PL}(4)) \rightarrow \pi_{3}(\mathrm{TOP}(5) / \mathrm{PL}(5))$ is an isomorphism, every closed topological 5-manifold is a handlebody.

(2) If $\pi_{3}(\mathrm{TOP}(4) / \mathrm{PL}(4)) \rightarrow \pi_{3}(\mathrm{TOP}(5) / \mathrm{PL}(5))$ is onto then every oriented closed topological 5-manifold is a handlebody.

(Note that (2) above would imply that Rohlin's theorem fails for TOP.)

One can get results reminiscent of Theorem 5.17 for $s$-cobordisms between 4-manifolds as well. If $W, W^{\prime}$ are $s$-cobordisms from a compact topological 4-manifold to a smooth manifold we say that $W$ is equivalent to $W^{\prime}$ if there are smooth $s$-cobordisms $V$ and $V^{\prime}$ with $\partial_{2} W=\partial_{1} V, \partial_{2} W=\partial_{1} V^{\prime}$ and a homeomorphism of $W \cup V$ onto $W^{\prime} \cup V^{\prime}$ which is the identity on $M=\partial_{1} W$ $=\partial_{1} W^{\prime}$ and a diffeomorphism from $\partial_{2} V$ to $\partial_{2} V^{\prime}$.

THEOREM 5.26 [Sch 2]. There is a $k$ such that for any connected compact topological manifold $M$, equivalence classes of s-cobordisms of $M \# k\left(S^{2} \times\right.$ $\left.S^{2}\right)$ to a smooth manifold are in 1-1 correspondence with elements of $H^{3}\left(M ; Z_{2}\right)$. If $M$ is orientable then $k$ may be taken to be 1 .

\section{CHAPTER 6. SURGERY IN DIMENSION 4}

6.1 Obstructions to four-dimensional surgery. It is clear from our discussion in the previous chapter that although surgery theory has greatly advanced our knowledge of the classification of PL-structures on topological manifolds in high dimensions it has contributed essentially nothing to the resolution of such questions in dimension 4. Furthermore no other techniques have managed to tell us anything either.

Another application of surgery theory which has yielded high caliber results has been that of the existence and classifications of PL or smooth manifold structures of a given homotopy type. These results are dependent of course on working with the spaces $G / \mathrm{PL}$ and $G / \mathrm{O}$. For the case of simplyconnected homotopy types we have, for example, the following results.

THEOREM I [Br 1], [N]. Let $\left(f_{i}, b_{i}\right), i=0,1$ be normal maps $f_{i}: M_{i}^{m} \rightarrow X, X$ a 1-connected Poincaré complex of dimension $m>4$ and suppose $f_{0}, f_{1}$ are homotopy equivalences.

If $f_{0}$ is normally cobordant to $f_{1}$ then (1) $M_{0}$ is $h$-cobordant to $M_{1}$ in the PL or TOP category. 
(2) In DIFF, if $m$ is even, $M_{0}$ is smoothly $h$-cobordant to $M_{1}$, while if $m$ is odd, $M_{0}$ is smoothly $h$-cobordant to $M_{1} \# \Sigma$, for some homotopy sphere $\Sigma=\partial U, U$ a smooth parallelizable $(m+1)$-manifold.

COROLlARY II. The integral cohomology ring and rational Pontryagin classes determine a smooth simply-connected manifold $M^{n}$ to within a finite number of possibilities for $n \geqslant 5$. If the groups $H_{4 i}\left(M^{n}\right)$ are finite for $0<4 i<n$ then there exist a finite number of smoothness structures on the topological manifold $M^{n}$.

One can also get specific results on the existence of fake manifolds of the homotopy type of a real or complex projective space or of lens spaces. See [Wa 3] for a discussion and references.

Although neither Theorem I and its corollary nor the analogues of the results of [Wa $3, \S \S 14,15]$ on fake projective spaces, fake lens spaces and fake tori are known to be true in dimension 4, they do indicate what types of phenomena we should look for.

For example if surgery theory were to work in dimension 4 as in higher dimensions we would be able to deduce the existence of an almost parallelizable TOP manifold $W^{4}$ with definite bilinear form of signature 8. Similarly the existence of $\mathrm{PL} 4$-manifolds realizing $E_{8} \oplus E_{8}$ as well as $E_{16}$ could be guaranteed.

The problem which prevents automatic extension of higher-dimensional surgery techniques to dimension 4 is the same obstacle encountered in trying to extend the proof of the $h$-cobordism theorem to dimension 4 . That is, the failure of the Whitney trick for codimension 2 submanifolds of 4-manifolds. In particular there exist homology classes $\xi \in H_{2}(M ; Z)$ of simply-connected 4-manifolds which can not be represented by (smoothly) embedded spheres! Thus one can not even begin to try and do surgery on these classes.

The existence of nonrepresentable homology classes can be immediately seen as a consequence of Corollary 1.13 [KM 1, Theorem 1] which we recall.

COROllary 1.13. Let $M^{4}$ be a closed, orientable PL 4-manifold and suppose $\xi \in H_{2}(M ; Z)$ is characteristic (i.e. its reduction $\bmod 2$ is dual to the second Steifel-Whitney class $w_{2}(M) \in H^{2}(M ; Z)$ of $M$, or equivalently $\xi \cdot \xi+\xi \cdot \phi \equiv$ $0(\bmod 2)$ for all $\left.\phi \in H_{2}(M)\right)$. Then if $\xi$ is representable by a locally-flat 2-sphere we have $\xi \cdot \xi-\sigma(M) \equiv 0(\bmod 16)$.

We thus deduce:

PROPOSITION 6.1. There exists a closed simply-connected PL 4-manifold $M^{4}$ and $\xi \in \pi_{2}\left(M^{4}\right)$ such that $\xi$ is not representable by a locally flat embedded sphere. In particular if $M^{4}=S^{2} \times S^{2}$ and $\alpha, \beta$ are the standard generators of $\mathrm{H}_{2}\left(S^{2} \times S^{2} ; \mathrm{Z}\right) \approx \pi_{2}\left(S^{2} \times S^{2} ; \mathrm{Z}\right)$ or $M^{4}=\mathrm{C} P^{2}$ and $\gamma$ is the standard generator of $\mathrm{H}_{2}\left(\mathrm{CP}^{2}\right)$ then neither $\pm 2(\alpha+\beta)$ nor $\pm 3 \gamma$ can be so represented.

Proof. For $S^{2} \times S^{2}$ we have $[2(\alpha+\beta)]^{2}=8$ which clearly is not congruent to $\sigma\left(S^{2} \times S^{2}\right)=0(\bmod 16)$ and for $C P^{2}$ we have $[3 \gamma]^{2}=9$ which is not congruent to $\sigma\left(\mathbf{C} P^{2}\right)=1(\bmod 16)$.

Actually 1.13 implies that if $M=S^{2} \times S^{2}$ and $\xi=p \alpha+q \beta \in H_{2}(M ; Z)$ then whenever $p, q$ are even, $\xi$ is characteristic, while if $p \equiv q \equiv 2(\bmod 4)$ 
then $\xi^{2} \equiv 8(\bmod 16)$ and so cannot be represented. Similarly $r \gamma$ with $r= \pm 3 \bmod 8$ can not be represented. (Results of Tristram [Tr], Rohlin [R 2] and Hsiang and Sczarba [HSZ] show that if g.c.d. $(p, q)>1$ in $\xi=p \alpha+q \beta$ or $|r| \geqslant 3$ in $r \gamma$ then neither $\xi$ nor $r \gamma$ can be represented by locally-flat embedded spheres.)

Knowing that all elements of $\mathrm{H}_{2}(M)$ can not be represented by smoothly or locally-flat embedded spheres it is of interest to determine what is the minimal possible genus of an orientable surface $F_{g}$ representing a given element $\xi \in \mathrm{H}_{2}(M)$. In addition, though also not useful for purposes of surgery, one can also ask: when is $\xi \in H_{2}(M)$ representable by a not necessarily locally-flat embedded sphere? In [KM 1] Kervaire and Milnor show, for example, that all elements in $\mathrm{H}_{2}\left(\mathrm{~S}^{2} \times \mathrm{S}^{2}\right)$ and $\mathrm{H}_{2}\left(\mathrm{CP}^{2}\right)$ can be so represented! We discuss both of these questions in some detail in Chapter 7.

We note that the above proposition depended on Rohlin's theorem and thus on the fact that $M$ was smooth (= PL in dimension 4). Questions about 4-dimensional topological manifolds are even more delicate than about PL ones and we thus restrict attention to the PL (= smooth) category in the discussion which follows.

6.2 Four-dimensional analogues of high-dimensional surgery theory. As a consequence of Proposition 6.1 it is clear that much more care must be exercised in trying to do 4-dimensional surgery than in higher dimensions. There are then two alternate approaches to extending higher-dimensional results to dimension 4 . One can either: (1) attempt to use alternative arguments not directly involving embedding questions to deduce 4-dimensional extensions of the basic high-dimension surgery theorems, or (2) discover which elements of $\mathrm{H}_{2}\left(M^{4}\right)$ are representable by embedding $f: S^{2} \times D^{2} \rightarrow M^{4}$ and build a new theory restricting consideration to those elements.

We shall discuss both approaches in what follows: In both methods progress depends strongly on understanding $\operatorname{Isom}(M)=\{$ auto PL-homeomorphisms of $M$ \} and $\operatorname{SE}(M)=$ simple self-homotopy equivalences of $M$ \}.

The basic tool in the extension of Theorem 5.9 to dimension 4 is then:

Lemma 6.2 (Novikov, WALL) [N], [Wa 3, Chapter 14]. Let $X$ be a compact connected oriented PL 4-manifold with $H_{2}\left(\pi_{1}(X): Z_{2}\right)=0$.

Then every element in $\mathcal{T}_{4}(X, \partial X)$ with surgery obstruction zero is realizable by an element of $\operatorname{SE}(X, \partial X)$ (where in the relative case $\partial X \neq \varnothing$ we demand that $f \in \operatorname{SE}(X, \partial X)$ is an isomorphism on $\partial X)$.

Proof. We shall begin by supposing that $\pi_{1}(X)=0$ and then indicate the necessary modifications if only $H_{2}\left(\pi_{1}(X), \mathbf{Z}_{2}\right)=0$. We must first calculate what $\operatorname{Ker} \sigma, \sigma: \mathcal{T}_{4}(X, \partial X) \rightarrow L_{4}(1)$ is.

Using the methods of $[\mathrm{Br} \mathbf{1}],[\mathbf{S 1}, 2]$ it can be shown that $\mathcal{T}_{4}(X, \partial X) \simeq$ $[X / \partial X, G / \mathrm{PL}]$ is isomorphic to $\operatorname{Hom}\left(H_{2}\left(X, \partial X ; \mathbf{Z}_{2}\right)^{\perp}, \mathbf{Z}_{2}\right) \oplus \mathbf{Z}$, where $H_{2}\left(X, \partial X ; \mathbf{Z}_{2}\right)^{\perp}=\operatorname{Ker} w_{2}(X), w_{2}(X): H_{2}\left(X, \partial X ; \mathbf{Z}_{2}\right) \rightarrow \mathbf{Z}_{2}$ the second Stiefel-Whitney class of $X$. Furthermore $\sigma$ is essentially projection to $\mathbf{Z} \approx$ $L_{4}(1)$.

Now by [Thm] we can represent a basis of $H_{2}\left(X, \partial X ; \mathbf{Z}_{2}\right)^{\perp}$ by 2-submanifolds $V_{i}^{2}$ of $X$. Then ker $\sigma$ corresponds to all possible assignments of elements 
of $\mathbf{Z}_{2}$ to the $V_{i}$. To proceed we must see precisely how Ker $\sigma$ corresponds to $\operatorname{Hom}\left(H_{2}\left(X, \partial X ; \mathbf{Z}_{2}\right)^{\perp}, \mathbf{Z}_{2}\right)$.

Thus suppose $(f, b)$ is a normal map, $f:(M, \partial M) \rightarrow(X, \partial X)$, in $\mathcal{T}_{4}(X, \partial X)$. Use the graph of the embedding $V_{i} \rightarrow X$ to consider $V_{i}$ as a submanifold of $X \times V_{i}$ and let $f_{i}:(M, \partial M) \times V_{i} \rightarrow(X, \partial X) \times V_{i}$ be $f \times \operatorname{id}_{V_{i}}$. Then we can use transversality and elementary surgery to deform $f_{i}$ so that (1) it is transverse regular to $V_{i}$ and (2) $U_{i}=f_{i}^{-1}\left(V_{i}\right)$ is a submanifold of $(M, \partial M) \times$ $V_{i}$ and $\phi_{i}=f_{i} \mid U_{i}$ is 2 -connected. Then $\phi_{i}$ defines a normal class $\left[\phi_{i}\right]$ in $\mathcal{T}_{2}\left(V_{i}\right)$ and we define $\phi(f): H_{2}\left(X, \partial X ; \mathbf{Z}_{2}\right)^{\perp} \rightarrow \mathbf{Z}_{2}$ by $\phi(f)\left(V_{i}\right)=\sigma\left(\left[\phi_{i}\right]\right) \in L_{2}(1) \approx$ $\mathbf{Z}_{2}$. (The $Z$-factor in $\mathscr{T}_{4}(X, \partial X)$ is given by $\operatorname{Index}(X)-\operatorname{Index}(M)$ (or $\frac{1}{2}$ that difference if $w_{2}(X)=0$ ), as in [ $\left.\mathrm{Br} \mathbf{1}\right],[\mathbf{N}]$.)

Now to show that $\operatorname{SE}(X, \partial X) \rightarrow \operatorname{Ker} \sigma$ is onto we must show that any assignment of elements of $\mathbf{Z}_{2}$ to the $V_{i}$ is induced by a self-homotopy equivalence of $X$. We begin by choosing an embedding of $D^{4} \rightarrow X$. Shrink $D^{4}$ to a point to get a map $c: X \rightarrow X \vee S^{4}$. Recall that $\pi_{4}\left(S^{2}\right) \approx \mathbf{Z}_{2}$ and let $\eta$ represent the generator.

Suppose $\alpha: S^{2} \rightarrow X$ represents an element of $\pi_{2}(X)$ and set $F_{\alpha}$ equal to the composite map

$$
X \stackrel{c}{\rightarrow} X \vee S^{4} \stackrel{1 \vee \eta}{\rightarrow} X \vee S^{2} \stackrel{(1, \alpha)}{\rightarrow} X
$$

Clearly $F_{\alpha}$ induces the identity on $H_{*}(X)$ and thus is a homotopy equivalence. (In fact it is easy to see that even if $X$ is not simply-connected $F_{\alpha}$ will induce the identity on $\pi_{*}(X)$ and thus by Theorem 5.5 be a homotopy equivalence in all cases.) To see what element of $\mathcal{F}_{4}(X, \partial X)$ corresponds to $F_{\alpha}$ we set $f=F_{\alpha}$ and calculate $\sigma\left(\left[\phi_{i}\right]\right)$ for all the $V_{i}$. In our case this can be done by noting that we may assume without loss of generality that $V_{i}$ is disjoint from $D^{4}$ so that $F_{\alpha}^{-1}\left(V_{i}\right)=V_{i} \cup W$, with $W$ the framed submanifold of $D^{4}$ obtained as the preimage of the points of the transversal intersection of $\alpha\left(S^{2}\right)$ and $V$. Then $\alpha\left(\left[\phi_{i}\right]\right)$ is just $\operatorname{Arf}\left(W, D^{4}\right)$.

Now if $\alpha\left(S^{2}\right)$ intersects $V_{i}$ transversely in $\eta_{i}$ points, then each point has preimages with Arf invariant one and so $\sigma\left(\left[\phi_{i}\right]\right) \equiv[\alpha] \cdot V_{i} \bmod \mathbf{Z}_{2}$, where $[\alpha]$ is the class in $H_{2}(X)$ defined by $\alpha(X)$. In particular then, all of Ker $\sigma$ can be realized by elements of $\operatorname{SE}(X)$ if and only if all the elements of $H_{2}(X ; Z)$ are realizable by elements of $\pi_{2}(X)$. This however follows by Hurewicz's theorem.

If $X$ is not simply-connected the question of realizing elements in Ker $\sigma$ by those of $\operatorname{SE}(X)$ again boils down to the question of realizing elements of $H_{2}\left(X ; Z_{2}\right)$ (more precisely of $H_{2}(X ; Z)^{\perp}$ ) by elements of $\pi_{2}(X)$.

Using the homology sequence associated to the universal covering manifold $\tilde{X}$ of $X$, we obtain the exact sequence:

$$
H_{2}\left(\tilde{X} ; \mathbf{Z}_{2}\right) \rightarrow H_{2}\left(X ; \mathbf{Z}_{2}\right) \rightarrow H_{2}\left(\pi_{1}(X) ; \mathbf{Z}_{2}\right) \rightarrow 0 .
$$

Using Hurewicz's theorem we obtain that $\pi_{2}(\tilde{X}) \rightarrow H_{2}(\tilde{X})$ is onto while $\pi_{2}(\tilde{X})=\pi_{2}(X)$. Thus if $H_{2}\left(\pi_{1}(X), \mathbf{Z}_{2}\right)=0$ we can again realize all elements of $H_{2}(X)$ by maps $S^{2} \rightarrow X$, and therefore $H_{2}\left(\pi_{1}(X) ; Z_{2}\right)=0$ suffices to guarantee that $\operatorname{SE}(X) \rightarrow \mathcal{T}_{4}(X)$ is onto $\operatorname{Ker} \sigma$.

An immediate consequence of Lemma 6.2 is then: 
TheOREM 6.3 (Novixov, WALL) [N], [Wa 3]. Let $X$ be a compact connected oriented PL 4-manifold with $H_{2}\left(\pi_{1}(X) ; \mathbf{Z}_{2}\right)=0$.

Then

$$
\delta_{4}(X, \partial X) \stackrel{\eta}{\rightarrow} \mathcal{T}_{4}(X, X) \stackrel{\sigma}{\rightarrow} L_{4}\left(\pi_{1}(X)\right)
$$

is exact.

If furthermore $\partial X=\varnothing$ and every element of $L_{5}\left(\pi_{1}(X)\right)$ is realizable by an element in $\Omega_{5}^{+}(X \times[I, 0,1])$, then

$$
\delta_{5}(X \times I, X \times \partial I) \stackrel{\eta}{\rightarrow} \mathcal{\sigma}_{5}(X \times I, X \times \partial I) \stackrel{\sigma}{\rightarrow} L_{5}\left(\pi_{1}(X)\right) \stackrel{\omega}{\rightarrow} \delta_{4}(X) \stackrel{\eta}{\rightarrow} \sigma_{4}(X)
$$

is also exact.

PRoof. The exactness of (**) can be deduced from the proofs of Theorems 5.7 and 5.9. All the surgery questions encountered are essentially higher-dimensional absolute surgery problems and can be handled by such techniques. The proof of (*) is of course established by Lemma 2 since $\operatorname{SE}(X, \partial X) \hookrightarrow$ $\delta_{4}(X, \partial X)$.

We thus have the following generalization of Theorem 1.2.

THEOREM 6.4. Let $X, Y$ be closed connected oriented PL 4-manifolds with $H_{2}\left(\pi_{1}(X) ; Z_{2}\right)=0$ and suppose the map $\sigma$ in $(* *)$ above is onto. Then if $X$ is simple homotopy equivalent to $Y$ it is s-cobordant to $Y$.

Proof. Let $f: Y \rightarrow X$ be a simple homotopy equivalence. By Lemma 6.2 there exists a (simple) self-homotopy equivalence $j: X \rightarrow X$ having the same normal invariants as $f$. Thus $f$ and $j$ are normally cobordant. But the assumption on $\sigma$ guarantees the exactness of $(* *)$ and since $\sigma$ is onto $\eta$ must be injective. Thus $f$ must be $s$-cobordant to some (simple) self-homotopy equivalence $j^{\prime}: X \rightarrow X$ and so $Y$ is $s$-cobordant to $X$.

Corollary 6.4' [Cap 1, P. 164]. Suppose $X, Y$ are simple homotopy equivalent closed connected oriented PL 4-manifolds. Suppose $\pi_{1}(X)$ is a finite group of odd order or zero.

Then $X$ is s-cobordant to $Y$. In particular simply-connected homotopy equivalent (closed connected PL) 4-manifolds are h-cobordant.

Proof. By a straightforward computation $H_{2}\left(\pi_{1}(X) ; Z_{2}\right)=0$ while by [Bak], [Wa 7] $L_{5}\left(\pi_{1}(X)\right)=0$. Thus the conditions for Theorem 6.4 are satisfied. (We note that Novikov's proof of Theorem 2 of $\$ 1$ was essentially by use of the above methods.)

REMARK. Not all groups $G$ satisfying $H_{2}\left(G ; \mathbf{Z}_{2}\right)=0$ also satisfy $L_{5}(G)=0$. For example $G=\mathbf{Z}$ satisfies $H_{2}\left(G ; Z_{2}\right)=0$ but $L_{5}(Z) \approx Z$ [Wa 3], [Sh 1], [Sh 3]. We note that $H_{2}\left(G ; Z_{2}\right)=0$ whenever $G$ is the fundamental group of a $\mathbf{Z}_{2}$-homology sphere and thus a large class of groups have this property. However $\boldsymbol{H}_{2}\left(\mathbf{Z}^{4} ; \mathbf{Z}_{2}\right) \neq \varnothing$ and $\boldsymbol{H}_{2}\left(\mathbf{Z}_{2} ; \mathbf{Z}_{2}\right) \neq \varnothing$. The above techniques break down completely then for such spaces as $T^{4}$ or $\mathbf{R} P^{4}$ and we must turn to approach (2) to make any progress. We thus consider some approaches to the problem of embedding spheres in 4-manifolds. 
Proposition 6.5. Let $P=\mathbf{C} P^{2}$ and let $M$ be an arbitrary oriented connected compact 4-manifold. Then

(1) (See [KM 1].) If $\gamma_{1}, \gamma_{2}$ are the canonical generators of $P \# P$ then $3 \gamma_{i} \in H_{2}(P \# P)$ is representable by a smoothly embedded sphere.

(2) (See [Bord].) If $M_{k}=\#_{i=1}^{k} P$ with canonical homology generators $\gamma_{i}$, then $\pm n \gamma_{i}$ is representable by a smoothly embedded sphere in $M_{k}, k=\frac{1}{2}\left(n^{2}-3 n+\right.$ 4).

(3) (See [Wa 1].) If $f: S^{2} \rightarrow M$ is a smooth embedding representing $\gamma \in$ $\mathrm{H}_{2}(\mathrm{M} ; \mathrm{Z})$. Then

(i) If $\gamma^{2}=0$ any $n \gamma$ is representable by a smoothly embedded sphere.

(ii) If $\gamma^{2}= \pm 1$ then $2 \gamma$ is representable by a smoothly embedded sphere.

Proof. We shall begin by proving (3) to indicate the type of technique involved.

First note that if $\gamma^{2}=0$ then $f\left(S^{2}\right)$ has trivial normal bundle in $M$ and one can extend the embedding $f: S^{2} \rightarrow M$ to $F: S^{2} \times D^{2} \rightarrow M$. Then taking $n$-disjoint copies of embedded $S^{2}$ s, $F\left(S^{2} \times x_{i}\right)$, and 'piping' them together gives the desired representation of $n \gamma$.

If $\gamma^{2}= \pm 1$ then $f\left(S^{2}\right)$ has a normal bundle $N$ which is a Hopf-disc bundle $f\left(S^{2}\right)$. Thus there exists a cross section $\Sigma$ of $N$ in $M$ which is an embedded sphere intersecting $f\left(S^{2}\right)$ transversely in one point $p$. Taking out a neighborhood of $p$ in $f\left(S^{2}\right)$ and $\Sigma$ and piping the resulting $S^{2}$ - (2-discs) together gives a 2 -sphere representing $2 \gamma$.



FigURE 6.1A
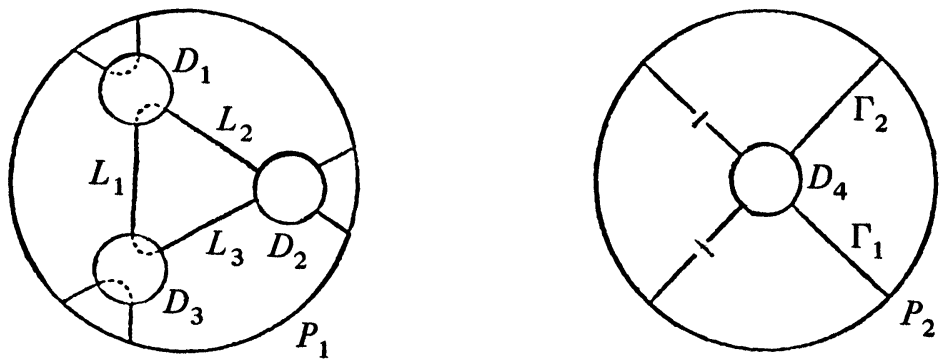

FIGURE 6.1B 
To prove (1) (we leave (2) to the reader) one uses a similar technique. We can represent $3 \gamma_{1}$ as the union of three lines $L_{i}$ in $P_{1}$ in general position as in Figure 6.1A with transversal intersection $L_{1} \cap L_{2}=\left\{X_{1}\right\}, L_{2} \cap L_{3}=\left\{X_{2}\right\}$, $L_{3} \cap L_{1}=\left\{X_{3}\right\}$ with all $X_{i}$ distinct. Now take lines $\Gamma_{1}, \Gamma_{2}$ representing $\gamma_{2},-\gamma_{2}$ in $P_{2}$ and intersecting transversely in the unique point $y \in \Gamma_{1} \cap \Gamma_{2}$. Take out neighborhoods of $X_{1}$ in $L_{1}$ and $L_{2}, X_{2}$ in $L_{2}$ and $L_{3}$. We can pipe $L_{1}$ and $L_{2}$ together and $L_{2}$ and $L_{3}$ together to get a sphere $\sigma$ with one self-intersection point $X_{3}$ representing $3 \gamma$, (see Figure 6.1B). Now take out a 4 disc $\Delta_{1}$ about $X_{3}$ in $P_{1}$ and $\Delta_{2}$ about $y$ in $P_{2}$ and form their connected sum $\overline{P_{1}-\Delta_{1}} \cup \overline{P_{2}-\Delta_{2}}$, connecting one 'sheet' of $\overline{\sigma-\sigma \cap \Delta_{1}}$ with $\overline{\Gamma_{1}-\Gamma_{1} \cap \Delta_{2}}$ and the other with $\overline{\Gamma_{2}-\Gamma_{2} \cap \Delta_{2}}$. This gives a sphere representing the cycle $3 \gamma_{1}+\gamma_{2}-\gamma_{2}=3 \gamma_{2}$ in $P_{1} \# P_{2}$.

6.3 Simply-connected four-dimensional (stable) surgery. The above type of construction proves useful in a more systematic approach to 4-dimensional surgery developed by Wall in [Wa 1]. In particular recall that a homology class $a \in H_{2}(M)$ is called ordinary if it is not characteristic and is called primitive if there exists $b \in H_{2}(M)$ with $a \cdot b= \pm 1$.

Then Wall shows

Theorem 6.6 (Wall), [Wa 1]. Suppose $N$ is a simply-connected closed oriented smooth 4-manifold with indefinite form $L_{N}$. Let $M=N \# S^{2} \times S^{2}$.

Then every primitive, ordinary element of $H_{2}(M)$ is represented by an imbedded 2-sphere.

Theorem 6.6 is proven by examining the nature of the diffeomorphisms of $M$ and showing that given any primitive ordinary element $\xi \in H_{2}(M)$ there is a class $X \in H_{2}(M)$ representable by an embedded sphere in the $\# S^{2} \times S^{2}$ portion of $M$ and a diffeomorphism $f: M \rightarrow M$ taking $X$ to $\xi$. More precisely use is made of:

TheOREM 6.7 (WALl [Wa 1]). Let $N, M$ be as in Theorem 6.6 above. Suppose $\phi$ is a ring automorphism of $H_{*}(M)$. Then there exists an autodiffeomorphism $f$ of $M$ with $f_{*}=\phi$.

(Note that a ring automorphism $\phi$ of $H_{*}(M)$ is equivalent to a group automorphism $\tilde{\phi}: H_{2}(M) \rightarrow H_{2}(M)$ satisfying $\tilde{\phi}(x) \cdot \tilde{\phi}(y)=x \cdot y$.) We shall first use Theorem 6.7 to prove Theorem 6.6 and then sketch a proof of Theorem 6.7.

Proof OF TheOReM 6.6. Let $\xi \in H_{2}(M)$ be primitive and ordinary and suppose $\xi^{2}=r$.

(1) There exist some $x_{r} \in H_{2}(M)$, primitive and ordinary with $x_{r}^{2}=r$ such that $x_{r}$ is representable by a smoothly embedded 2-sphere.

Proof OF (1). First suppose $r$ is even say $r=2 k$. Let $x_{0}$ and $y$ represent the canonical zero section and fiber of $S^{2} \times S^{2}$ considered as elements of $\mathrm{H}_{2}\left(\mathrm{~N} \# \mathrm{~S}^{2} \times S^{2}\right)$. Clearly both $x_{0}, y$ are representable by smoothly embedded spheres. Define $x_{r}=x_{0}+k y$. Since $y^{2}=0$ we can take $|k|$ disjoint 2-spheres $\left(S^{2}\right)_{i}, i=1, \ldots, k$, sitting as fibers with appropriate orientations in $S^{2} \times S^{2} \hookrightarrow M$ such that $k y$ is homologous to the union of the $\left(S_{i}^{2}\right)$. Each $\left(S_{i}^{2}\right)$ 
intersects the base $B$ of $S^{2} \times S^{2}$ (which is an embedded sphere representing $X_{0}$ ) transversely in one point and we can then replace each intersection point $x_{i}$ by a pipe connecting $S_{i}^{2} \cap \partial \Delta_{i}$ to $B \cap \partial \Delta_{i}$, where $\Delta_{i}$ is a 4-disc in $M$ about $x_{i}$, as we did in Proposition 6.5. We thus get an embedded sphere representing $x_{r}$. Then $x_{r}^{2}=\left(x_{0}^{2}+2 k x_{0} \cdot y+y^{2}\right)=2 k$ since $x_{0} \cdot y=1$ and $y^{2}=x_{0}^{2}=0$. If $r$ is odd then $L_{M}$ is of type $I$ and as we noted in Chapter 1 we have $M=N \# S^{2} \times S^{2}=N \# S^{2} \times S^{2}$. If $x_{0}, y$, now denote the cross section and fiber of $S^{2} \underset{\sim}{\times} S^{2}$, again considered as elements of $H_{2}\left(N \# S^{2} \underset{\sim}{\times} S^{2}\right)$, and $r=2 k+1$ then $x_{r}=x_{0}+k y$ can, precisely as above, be represented by an embedded sphere. However now since $x_{0}^{2}=1, x_{0} \cdot y=1, y^{2}=0$ we find $\left(x_{0}^{2}+2 k x_{0} \cdot y+y^{2}\right)=2 k+1$ as desired.

We now need the following algebraic fact proved in [Wa 8].

(2) Let $(H, \cdot)$ be a symmetric inner-product space over $\mathbf{Z}$. Suppose $x, y$ are primitive, ordinary elements of $H$. Then if $b=\operatorname{rk}(H)$ and $\sigma=\sigma(H, \cdot)$ then $b-|\sigma| \geqslant 4$ implies there exists an isometry $\phi$ of $(H, \cdot)$ such that $y=\phi(x)$ (i.e. the isometry group $D(H)$ is transitive on primitive, ordinary elements provided $b-|\sigma| \geqslant 4)$.

Applying (2) we note that if $L_{N}$ is indefinite then $b_{2}(M)-|\sigma(M)| \geqslant 4$ and thus there is a ring homomorphism $\phi: H_{*}(M) \rightarrow H_{*}(M)$ with $\xi=\phi\left(x_{r}\right)$. But, by Theorem 6.7, $\phi$ is induced by an automorphism $f \in \operatorname{DIFF}(M)$ and thus since $x_{r}$ is representable by an embedded sphere so is $\xi$.

(Note that in our arguments above we only used the nonsingularity of $L_{N}$ which is guaranteed under the sole condition that $H_{1}(\partial N)=0$.)

Proof of THEOREM 6.7. We again need an algebraic fact first proved by Wall. Let $(H, \cdot)$ be an inner-product space over $\mathbf{Z}$ and let $X=H \oplus U$ be the direct sum space ( $U$ the hyperbolic inner product space with form represented by $\left(\begin{array}{l}0 \\ 1 \\ 1\end{array}\right)$ as in Chapter 1). Let $x, y$ denote a canonical basis for $U$ (i.e. $\left.x^{2}=y^{2}=0 ; x \cdot y=+1\right)$. If $Y$ is an arbitrary inner-product space with $\omega \in Y-\{0\}$ and $0<|(\omega \cdot \omega)| \leqslant 2$ define an isometry $\zeta \rightarrow \zeta-\frac{2}{\omega \cdot \omega}(\zeta \cdot \omega) \omega$ and call it $S(\omega)$ if $\omega^{2}=\omega \cdot \omega= \pm 1$ and $R(\omega)$ if $\omega^{2}= \pm 2$. For $X=H \oplus U$ as above and $\omega \in H$ with $\omega^{2}=2 N \in \mathbf{Z}$ define isometries $E_{\omega}^{1}, E_{\omega}^{2}$ of $X$ by

$$
\begin{array}{ccc}
E_{\omega}^{1}: \zeta & \rightarrow \zeta-(\zeta \cdot \omega) y, & E_{\omega}^{2}: \zeta \rightarrow \zeta-(\zeta \cdot \omega) x, \\
x \rightarrow x-N y, & x \rightarrow x, \\
y & \rightarrow y, & y \rightarrow y-N x .
\end{array}
$$

Then Wall shows [Wa 8]:

Proposition 6.8. Suppose $H$ is indefinite (or $b \leq 8)$. Then $\operatorname{Isom}(X)$ is generated by

(1) $E_{\omega}^{1}, E_{\omega}^{2}$ for all $\omega \in H$ with $\omega^{2}$ even

(2) $R(x+y), R(x-y)$

if $H$ is of type II

and by (1); (2) above and in addition

(3) $S(z)$ for some $z \in H$ with $z^{2}= \pm 1$, if $H$ is of type $\mathrm{I}$.

Thus to prove 6.7 it suffices to show that the above isometries can be realized by diffeomorphisms. Clearly the automorphisms $R(x+y), R(x-y)$ 
on $H_{*}\left(S^{2} \times S^{2}\right)$ are realizable by some autodiffeomorphisms, say $r_{x+y}, r_{x-y}$ of $S^{2} \times S^{2}$ and using the disc and isotopy extension theorem [RS] one sees that one can form the connected sum of diffeomorphisms and thus realize $R(x+y)$, on $H_{*}(M)$ by id ${ }_{N} \# r_{x \pm y}$ respectively.

In addition if $N$ has form $L_{N}$ of type I then $N \# S^{2} \times S^{2}=$ $N \# S^{2} \underset{\sim}{\times} S^{2}$ as in Chapter 1 and since $S^{2} \times S^{2}=P \# Q$, complex conjugation on $P$ provides a diffeomorphism inducing an isometry of type $S(z)$, $z^{2}= \pm 1$, on $H_{*}(M)$. Thus it suffices to show that we can realize all the $E_{\omega}^{1}$ by diffeomorphisms. (The realizability of $E_{\omega}^{2}$ would then follow automatically by interchanging factors in $S^{2} \times S^{2}$.)

Thus suppose $f_{0}: S^{1} \times D^{3} \rightarrow N$ is an embedding and $h_{t}$ is an isotopy of $f\left(S^{1} \times 0\right)$ in $N$ with initial and final position given by $f\left(S^{1} \times 0\right)$. Now $h_{1}$ will be an autodiffeomorphism of $N$ inducing (by the uniqueness of tubular neighborhoods) a bundle map $S^{1} \times D^{3}$ onto itself. Let $f_{1}: S^{1} \times D^{3}$ denote the embedding induced by $h_{1}$. Let $M_{0}$ be $N$ surgered along $f_{0}\left(S^{1} \times D^{3}\right)$ and $M_{1}$ be $N$ surgered along $f_{1}\left(S^{1} \times D^{3}\right)$. Then $h_{1}$ clearly induces a diffeomorphism $\hat{h}$ of $M_{0}$ onto $M_{1}$ (i.e. extend $h_{1} \mid N-f\left(S^{1} \times D^{3}\right)$ by the identity on $D^{2} \times S^{2}$ noting that setwise $\left.f_{0}\left(S^{1} \times D^{3}\right)=f_{1}\left(S^{1} \times D^{3}\right)\right)$.

Note that we have the following exact homology sequences (with integral coefficients) associated with the above surgeries $(i=1,2)$

$$
\begin{gathered}
0 \rightarrow H_{3}\left(N, N-f_{i}\left(S^{1} \times D^{3}\right)\right) \rightarrow H_{2}\left(N-f_{i}\left(S^{1} \times D^{3}\right)\right) \rightarrow H_{2}(N) \rightarrow 0 \\
0 \rightarrow H_{2}\left(N-f_{i}\left(S^{1} \times D^{3}\right)\right) \rightarrow H_{2}(M) \rightarrow H_{2}\left(D^{2} \times S^{2}, S^{1} \times S^{2}\right) \rightarrow 0
\end{gathered}
$$

with

$$
\begin{aligned}
H_{3}\left(N, N-f_{i}\left(S^{1} \times D^{3}\right)\right) & \approx H_{3}\left(S^{2} \times D^{3}, S^{1} \times S^{2}\right) \simeq \mathbf{Z} \\
H_{2}\left(D^{2} \times S^{2}, S^{1} \times S^{2}\right) & \approx \mathbf{Z} .
\end{aligned}
$$

Let $y_{i}$ be the image of a generator of $H_{3}\left(N, N-f_{i}\left(S^{1} \times D^{3}\right)\right)$ in $H_{2}(N-$ $\left.f_{i}\left(S^{1} \times D^{3}\right)\right)$ and let $x_{i}$ be the inverse image of a generator of $H_{2}\left(D^{3} \times\right.$ $\left.S^{2}, S^{1} \times S^{2}\right)$ in $H_{2}(M)$ constructed by taking the union of a surface $C$ in $N-f_{i}\left(S^{1} \times D^{3}\right)$ spanning $S^{1} \times 1$ and $D^{2} \times 1 \subset D^{2} \times S^{2} \subset M$. [C exists since $N$ and thus $N-S^{1}$ were 1-connected.]

Note that $x_{i} \cdot y_{i}=1, y_{i} \cdot y_{i}=0$ while $\left(x_{i}\right)^{2}=k_{i}$ can be anything. In fact if we let $U_{k_{i}}$ be a neighborhood of the wedge $x_{i} \vee y_{i}$ in $M_{i}$ (where we think of $x_{i}$, $y_{i}$ as cycles representing the appropriate homology classes) then $U_{k_{i}}$ is just the complement of a 4-disc in some $S^{2}$ bundle $T_{k_{i}}$ over $S^{2}$ and we have the geometric decomposition $M_{i}=N \# T_{k_{i}}$.

( $T_{k_{i}}$ can be thought of as the $S^{2}$ bundle over $S^{2}$ with structure group $\mathrm{SO}_{2}$ (rather than $\mathrm{SO}_{3}$ ) corresponding to the reduction given by the integer $k_{i}$ of $\mathrm{Z} \approx \pi_{1}\left(\mathrm{SO}_{2}\right) \rightarrow \pi_{1}\left(\mathrm{SO}_{3}\right) \approx \mathrm{Z}_{2}$. Then as manifolds $T_{2 k} \approx S^{2} \times S^{2}$ corresponding to the zero element of $\pi_{1}\left(\mathrm{SO}_{3}\right)$ while $T_{2 k+1} \approx S^{2} \underset{\sim}{\times} S^{2}$ corresponding to the nonzero element of $\pi_{1}$. As $\mathrm{SO}_{2}$ bundles $T_{k}$ has a canonical zero section denoted by $x_{k}$ and fiber denoted by $y_{k}$ satisfying $x_{k}^{2}=k, y_{k}^{2}=0, x_{k} \cdot y_{k}=1$ (note that $x_{k} \rightarrow x_{k}-k y_{k}, y_{k} \rightarrow y_{k}$ gives the diffeomorphism sending $T_{2 k}$ to $T_{0}$ and $T_{2 k+1}$ to $T_{1}$ for any $k$ ).)

Now let $\omega$ be the class of the surface in $N$ traced out by $f\left(S^{1} \times 0\right)$ under the isotopy $h_{t}$ and use the above geometric decomposition to identify $H_{2}(N)$ 
with an orthogonal direct summand of $H_{2}\left(M_{i}\right)$. Then if $\hat{h}_{*}$, the isomorphism on homology induced by the diffeomorphism $\hat{h}: M_{0} \rightarrow M_{1}$, is denoted by $E$ : $H_{2}\left(M_{0}\right) \rightarrow H_{2}\left(M_{1}\right)$, a straightforward calculation shows that $E(\xi)=\xi-(\xi$. $\omega) y_{1}, E\left(x_{0}\right)=x_{1}+\omega, E\left(y_{0}\right)=y_{1}$ for $\xi \in H_{2}(N)$, and $x_{i}, y_{i}$ as above.

Now if $\omega$ is any spherical class in $H_{2}(N)$ then we can easily find an isotopy of $S^{1}$ in $N$ with initial and final map representing $\omega$. [Proof: Let $g: S^{2} \rightarrow N$ represent $\omega$ and let $p: S^{1} \times S^{1} \rightarrow f\left(S^{1} \times 0\right) \subset N$ be the obvious projection map. Form the connected sum map $H$ of $S^{1} \times S^{1} \# S^{2} \approx S^{1} \times S^{1} \rightarrow N$ by attaching $f\left(S^{1} \times 0\right)$ to $g\left(S^{2}\right)$ via an $\operatorname{arc}$ in $N$. $H$ is clearly the desired isotopy.] Since $N$ is simply connected any $\omega \in H_{2}(N)$ is spherical and we thus have proven

Proposition 6.9 [Wa 1, TheORem 1]. Let $N$ be a simply-connected 4-manifold, $\omega \in H_{2}(N)$ with $\omega^{2}=r$. There is a diffeomorphism of $N \# T_{k+r}$ onto $N \# T_{k}$ inducing $\xi \rightarrow \xi-(\xi \cdot \omega) y_{k}^{\prime}, x_{k+r}^{\prime} \rightarrow x_{k}^{\prime}+\omega, y_{k+r}^{\prime} \rightarrow y_{k}^{\prime}$ (where the $x_{i}^{\prime}, y_{i}^{\prime}$ represent the obvious images of the classes $x_{i}, y_{i}$ of $T_{i}$ in $N \# T_{i}$ ).

Corollary 6.10. Let $L_{N}$ be of type I. Then $N \# S^{2} \times S^{2} \approx$ $N \# S^{2} \underset{\sim}{\sim} S^{2}$.

Proof. Choose $\omega \in H_{2}(N)$ with $\omega^{2}$ odd in the proposition above.

Corollary 6.11. If $\omega^{2}=2 s, N \# T_{k}$ admits a diffeomorphism inducing $E_{\omega}^{1}$ : $\xi \rightarrow \xi-(\xi \cdot \omega) y, x \rightarrow x+\omega-s y, y \rightarrow y$ on $H_{2}\left(N \# T_{k}\right)$.

In particular Theorem 6.7 is proven.

Note. (1) We need only assume $H_{1}(\partial N)=0$, which assures the nonsingularity of $L_{N}$ to obtain Theorem 6.7. Thus Theorem 6.6 is also true under this weaker hypothesis.

(2) In Theorem 6.7 one could have replaced $L_{N}$ indefinite by $b_{2}(N)<8$ and obtained the same results. See [Wa 1]. However the analogue of Theorem 6.6 can not be obtained.

(3) Actually examining the proof of Theorem 6.7 one sees that all we actually used was $H_{1}(N)=0$ and $\omega$ spherical. These hypotheses are not enough, however, to prove Theorem 6.6 . What can be obtained by essentially the same argument is:

THEOREM 6.6'. Suppose $N$ is a closed oriented smooth 4-manifold with indefinite form $L_{N}$ such that $H_{1}(N)=0, \omega, \xi \in H_{2}(N)$ are spherical with $\omega \cdot \xi=1$ and $\omega^{2}$ even. Then $\xi$ can be represented by an embedded sphere in $N \# S^{2} \times S^{2}$.

(We will present a far-reaching generalization of Theorem $6.6^{\prime}$ due to Cappell and Shaneson in what follows (see Theorem 6.17).)

By similar methods one can show:

Proposition 6.12. Let $M$ be a connected sum of copies of $P, Q$ and $S^{2} \times S^{2}$ and suppose that $b_{2}(M)-|\sigma(M)|=2$ implies $b_{2}(M) \leqslant 10$. Then any automorphism of $H_{*}(M)$ can be represented by a diffeomorphism.

Recall that in our proof of Theorem 2 of $\$ 1$ we used the following without proof. 
LEMMA 6.13. Let $M$ be a connected sum of copies of $S^{2} \times S^{2}$ and $S^{2} \times S^{2}$. Let $K_{0}, K_{1}$ be subgroups of $H_{2}(M)$ satisfying $k=\mathrm{rk} K_{i}=\frac{1}{2} b_{2}(M), i=0, \widetilde{1}$, and $x, y \in K_{i} \Rightarrow x \cdot y=0, i=0,1$. Then there exists an autodiffeomorphism $f$ of $M$ with $f_{*}\left(K_{0}\right)=K_{1}$.

PRoOF. By Theorem 6.7 above it suffices to show that there exists an isometry of $H_{2}(M)$ throwing $K_{0}$ onto $K_{1}$. But by the above hypothesis $K_{i}$ is its own annihilator under $L_{M}$ and it is easy to show that there exists a basis $e_{1}^{i}, \ldots, e_{k}^{i}, f_{1}^{i}, \ldots, f_{k}^{i}$ of $H_{2}(M)$ with $\left\langle e_{1}^{i}, \ldots, e_{k}^{i}\right\rangle=K_{i}$, and $e_{r}^{i} \cdot f_{s}^{i}=\delta_{r s}$, $f_{r}^{i} \cdot f_{s}^{i}=0$ if $(r, s) \neq(1,1)$ and

$$
f_{1}^{i} \cdot f_{1}^{i}= \begin{cases}0 & \text { if } L_{M} \text { is of type II, } \\ 1 & \text { if } L_{M} \text { is of type I. }\end{cases}
$$

Then the map $e_{r}^{0} \rightarrow e_{r}^{1}, f_{r}^{0} \rightarrow f_{r}^{1}$ is the desired isometry. The crucial use of the above lemma in the proof of Theorem 1.2 indicates how our knowledge about embedded spheres can help us obtain surgery results in dimension 4. Extensions of Wall's approach by Cappell and Shaneson provide 'stable' analogues of Theorems 6.9 through 6.11 without any restrictions on $\pi_{1}(N)$.

6.4 Nonsimply-connected surgery in dimension 4. In [CS 1], Cappell and Shaneson prove the following 'stable' versions of the higher-dimensional surgery theorems.

Theorem 6.14 (Compare Theorem 5.7 of Chapter 5). Let $X$ be a smooth 4-manifold (alternatively a Poincaré pair of dimension 4 with $X$ connected and finite).

Suppose $(h, b):(W, \partial W) \rightarrow(X, \partial X)$ is a degree one normal map inducing a homotopy equivalence of boundaries. Then $\sigma(h, b) \in L^{h}\left(\pi_{1}(X) W\right)$ vanishes if and only if, for some $t \geqslant 0$,

$$
(h, b) \# t\left(S^{2} \times S^{2}\right)=\left(h \# \mathrm{id}_{t\left(S^{2} \times S^{2}\right)}, b \# \mathrm{id}_{t\left(S^{2} \times S^{2}\right)}\right)
$$

is normally cobordant rel $\partial$ to a homotopy equivalence.

Theorem 6.15 (COMPARE TheOREM 5.7' OF ChAPTER 5). Let $X$ be a smooth 4-manifold (alternatively $(X, Y)$ a Poincaré pair of dimension 4 with $X$ connected and finite) and suppose $\gamma \in L_{5}\left(\pi_{1}(X), W\right)$. Then if $K=M \# r\left(S^{2} \times\right.$ $\left.S^{2}\right)$, for $r$ sufficiently large, and $h:(K, \partial K) \rightarrow(X, \partial X)$ is a homotopy equivalence covered by a linear bundle map $c: \nu(M) \rightarrow \eta_{X}$. Then there is a normal cobordism, relative to the boundary $(F, B), F: W \rightarrow X \times I$, between $(h, c)=$ $\left(F\left|\partial_{-} W, B\right| \partial_{-}(W)\right)$ and $\left(F\left|\partial_{+} W, B\right| \partial_{+} W\right)$ such that

(i) $F \mid \partial_{+} W: \partial_{+} W \rightarrow X \times 1$ is a homotopy equivalence, and

(ii) the obstruction $\sigma(F, B)$ defined in view of (i) is precisely $\gamma$.

(In what follows when we speak of 'stabilization' we will mean connect summing with sufficiently many copies of $S^{2} \times S^{2}$.)

The key part of the proof of Theorem 6.14 is again an analysis of the diffeomorphisms of a 4-manifold $M$ analogous to what was done in Theorem 6.7 and Proposition 6.9 for simply-connected $M$. We discuss the highlights of this analysis. 
By way of terminology let $\Lambda=\mathbf{Z}\left[\pi_{1}(X)\right]$ denote the integral group ring of $M$ and let $\tilde{M}$ be the universal covering manifold of $M$. Note that $H_{2}(M$; $\Lambda)=H_{2}(\tilde{M} ; Z)$ has a $\Lambda$-module structure induced by the action of $\pi_{1}(M)$ (and thus $\Lambda$ ) on $M$. Let $\rho: H_{2}(M ; Z) \times H_{2}(M ; Z) \rightarrow Z$ denote the usual intersection pairing and for $\alpha, \beta \in H_{2}(M ; Z)$ let $(\alpha \cdot \beta)=\Sigma_{g \in \pi_{1} M} \rho(\alpha, g \beta) g$ (where we have fixed a base point $*$ in $M$ and a corresponding base point $\tilde{*}$ in $\tilde{M}$ lying over *. In what follows we shall not mention base points explicitly but assume they have always been appropriately chosen).

Now let $w: \pi_{1}(M) \rightarrow\{ \pm 1\}$ be the orientation map; i.e. $w(x)=1$ if and only if $x$ preserves orientation in $M$. Let ${ }^{-}$be the antiautomorphism of $\Lambda$ defined by $\left(\Sigma_{g} \lambda_{g} g\right)^{-}=\Sigma_{g} w(g) \lambda_{g} g^{-1}$. Then this pairing is $Z$-bilinear, and for $\tau \in \Lambda$,

(i) $(\tau \alpha) \cdot \beta=\tau(\alpha \cdot \beta)$ and (ii) $\beta \cdot \alpha=(\alpha \cdot \beta)^{-}$.

Let $w_{2}(M)=w_{2}:(M ; Z) \rightarrow Z_{2}$ be the second Stiefel-Whitney class, and let $H: \pi_{2}(M) \rightarrow H_{2}(M ; Z)$ be the Hurewicz homomorphism. Let $\alpha \in$ $\operatorname{ker}\left(w_{2} \circ H\right)$, and let $f: S^{2} \rightarrow M$ represent $\alpha$. Then $f^{*} \tau_{M}$ is trivial, where $\tau_{M}$ is the tangent bundle of $M$. Hence there is a bundle equivalence of $\tau_{S^{2}} \oplus \varepsilon^{2}$ with $f^{*} \tau_{M}, \varepsilon^{2}$ a trivial bundle; there are two such equivalences up to isotopy because $\pi_{2}(\mathrm{O}(4))=0$. It is easy to see that they both determine isotopic monomorphisms $\tau_{S^{2}} \rightarrow f^{*} \tau_{M}$. So, by immersion theory, we have an immersion $\hat{f}: S^{2} \rightarrow M$, representing $\alpha$, with trivial normal bundle; and this immersion is unique up to regular homotopy. Given such an immersion, we may take its self-intersection invariant as in [Wa 3, Chapter 5]; this determines a well-defined map $\mu: \operatorname{ker}\left(w_{2} \circ H\right) \rightarrow \Lambda / I$, where $I=\{\lambda-\bar{\lambda} \mid \lambda \in \Lambda\}$. The following properties are satisfied:

(iii) $\alpha \cdot \alpha=\mu(\alpha)+\overline{\mu(\alpha)}$; (note that the right-hand side is really a well-defined element of $\Lambda$ );

(iv) $\mu(\alpha+\beta)=\mu(\alpha)+\mu(\beta)+\alpha \cdot \beta(\bmod I)$; and

(v) $\mu(\lambda \alpha)=\bar{\lambda} \mu(\alpha)$.

(See [Wa 3] for more details.)

Note that using the Hurewicz theorem we obtain $H_{2}(\tilde{M} ; \mathbf{Z}) \approx \pi_{2}(\tilde{M}) \approx$ $\pi_{2}(M)$ so we can think of $w_{2} \circ H$ as being defined on $H_{2}(\tilde{M} ; Z)=H_{2}(M ; \Lambda)$. The following analogue of Proposition 6.9 is then needed to prove Theorem 6.14 .

Proposition 6.16 [CS 1, Theorem 1.5]. Let $M$ be a smooth compact connected 4-manifold and suppose $M=N \#\left(S^{2} \times S^{2}\right)$ for some smooth manifold $N$. Let $\omega \in H_{2}(M ; \Lambda)$ with $w_{2} H(\omega)=0$ and suppose $\lambda \in \Lambda$ is such that $\lambda \equiv \mu(\omega)(\bmod I)$.

Then there is a (basepoint-preserving) autodiffeomorphism $\phi$ of $M \#\left(S^{2} \times\right.$ $\left.S^{2}\right)$ which preserves local orientations and induces the identity on $\pi_{1}\left(M \#\left(S^{2}\right.\right.$ $\left.\times S^{2}\right)$ ) so that $\phi_{*}(e)=e+\omega-\lambda f, \phi_{*}(f)=f$ and $\phi_{*}(\xi)=\xi-(\xi \cdot \omega) f$ for $\xi \in H_{2}(M ; \Lambda)$ where e, $f$ in $H_{2}\left(M \#\left(S^{2} \times S^{2}\right), \Lambda\right)$ are the standard generators of the summand $\mathrm{H}_{2}\left(S^{2} \times S^{2}, \Lambda\right)$.

Using Proposition 6.16 one can prove analogues of Theorems 6.6, 6.6' and 6.7.

If $\xi \in H_{2}(M ; \Lambda)$ with $w_{2} H(\xi)=0$ we shall say $\xi$ is strongly primitive if there exists $\tau \in H_{2}(M ; \Lambda)$ with $\lambda(\xi)=1$ and $w_{2} H(\tau)=0$. Then one has 
TheOREM 6.17 (COMPARE WITH TheORems 6.6, 6.6') [CS 1, TheORem 2.2]. Let $M$ be a compact, connected smooth four-manifold of the form $N$ \# $\left(S^{2} \times\right.$ $\left.S^{2}\right)$. Let $\xi \in \mathrm{H}_{2}(M ; \Lambda)=\pi_{2}(M)$ with $w_{2} H(\xi)=0$ be strongly primitive and suppose $\mu(\xi)=0$.

Then $\xi$ is represented by a smooth embedding $S^{2} \hookrightarrow M \# S^{2} \times S^{2}$ with trivial normal bundle so that the inclusion $\left(M \# S^{2} \times S^{2}-S^{2}\right) \rightarrow\left(M \# S^{2}\right.$ $\times S^{2}$ ) induces an isomorphism of fundamental groups.

Furthermore if $\mu(\xi) \in \mathbf{Z} \subset \Lambda$ the same conclusion holds except that $S^{2}$ will no longer have a trivial normal bundle. In addition if $\xi \in H_{2}(N ; \Lambda)$ we need only assume $\xi$ is primitive.

Using 6.16 and 6.17 we sketch a proof of Theorem 6.14 as follows

Proof of THEOREM 14. We first note that by surgering embedded 1spheres, if necessary, we can always assume that $\pi_{2}(h)=0$. Then by the definition of $\sigma(h, b)$ it is clear that $\sigma(h, b)=\sigma\left[(h, b) \# t\left(S^{2} \times S^{2}\right)\right]$ so the vanishing of $\sigma(h, b)$ is clearly necessary for the conclusion of the theorem to be satisfied. Conversely suppose $\sigma(h, b)=0$ then (replacing $W$ by $W \# q\left(S^{2}\right.$ $\left.\times S^{2}\right)$ if necessary) we can assume that $K_{2}(W)=\operatorname{ker}\left(h_{*}: H_{2}(W ; \Lambda) \rightarrow\right.$ $\left.H_{2}(X ; \Lambda)\right)$ is a free $\Lambda$-module with basis $\xi_{1}, \ldots, \xi_{t}, \tau_{1}, \ldots, \tau_{t}$, such that $\lambda\left(\xi_{i}, \xi_{j}\right)=\lambda\left(\tau_{i}, \tau_{j}\right)=\mu\left(\xi_{i}\right)=\mu\left(\xi_{j}\right)=0,1 \leqslant i, j \leqslant t$, and $\lambda\left(\xi_{i}, \tau_{j}\right)=\delta_{i j}$. We may (by adding an additional $S^{2} \times S^{2}$ summand to $W$ if necessary) assume that $W=P \# S^{2} \times S^{2}$, for some $P$, and thus apply Theorem 17 to realize $\xi_{1}$ by a smoothly embedded sphere $S^{2} \subset W \# S^{2} \times S^{2}$ with trivial normal bundle as in that theorem. We can now use the framing on the normal bundle of $\xi_{1}$, $g: S^{2} \times D^{2} \rightarrow W \# S^{2} \times S^{2}$ to perform framed surgery as described in Chapter 5, killing $\left(\xi_{1}, \tau_{1}\right)$ and obtaining a normal cobordism (note that there is no problem in extending the framing $g$ since $\left.\pi_{2}(\mathrm{O}(k))=0\right)(H, B), H$ : $W \times I \cup_{(g, 1)} D^{3} \times D^{2} \rightarrow X$ between $(h, b)$ and a new degree 1 normal map $\left(h^{\prime}, b^{\prime}\right), h^{\prime}: W^{\prime} \rightarrow X$, where $W^{\prime}=\partial_{+}\left[W \times I \cup D^{3} \times D^{2}\right]=\operatorname{cl}\left(W \#\left(S^{2} \times\right.\right.$ $\left.\left.S^{2}\right)-g\left(S^{2} \times D^{3}\right) \cup_{g} D^{3} \times S^{1}\right)$. Since by Theorem 6.17

$$
\pi_{1}\left(W \# S^{2} \times S^{2}-g\left(S^{2} \times D^{2}\right)\right) \approx \pi_{1}\left(W \# S^{2} \times S^{2}\right)
$$

we can use van Kampen's theorem to see that $\pi_{1}(W) \approx \pi_{1}\left(W^{\prime}\right)$. [In fact we note that $W^{\prime}$ is actually diffeomorphic to $W$ since the way a sphere representing $\xi$ in Theorem 6.17 is obtained is via an automorphism of $W \# S^{2} \times S^{2}$ throwing $\left[S^{2} \times \cdot\right]$ onto $\xi$. Thus the surgery above essentially kills the $S^{2} \times S^{2}$ in $W \# S^{2} \times S^{2}$ giving us a diffeomorphism of $W^{\prime}$ to $W$.]

Now we can verify that $K_{2}\left(W^{\prime}\right)$ has the same form as $K_{2}(W)$ but with rank reduced by two.

We can then as in [Wa 3, Theorem 3.3] continue this process until all of $K_{2}$ is killed. This then gives the desired homotopy equivalence at the expense of having to add on an $S^{2} \times S^{2}$ summand for each $\xi_{i}$ in $K_{2}$ killed.

Note that our aside on the isomorphism of $W$ and $W^{\prime}$ shows that if $(h, b)$ is a normal map as above, $h:\left(W^{4}, \partial W\right) \rightarrow\left(X^{4}, \partial X\right)$. Then the vanishing of $\sigma(h, b)$ implies that for some $t \geqslant 0, r \geqslant 0, P \# r\left(S^{2} \times S^{2}\right)$ is homotopy equivalent to $X \# t\left(S^{2} \times S^{2}\right)$.

To prove Theorem 6.15 we proceed as follows. We first note that it suffices to prove Theorem 6.15 for $h=$ identity map and $X=M \# r\left(S^{2} \times S^{2}\right)$ since 
one can obtain more general $h$ by composition as in our discussion of the action of $L_{m+1}(\pi)$ on $\mathcal{S}_{m}^{H}(X)$ in Chapter 5 .

Now for some $r>0$ there exists $\alpha \in U_{r}(\Lambda)$ representing $\gamma$. Thus there exists a kernel $K_{r}=\left(K_{r}, \phi_{r}, \mu_{r}\right)$ of dimension $2 r$ with $\alpha$ an automorphism of $K_{r}$. Let $K=M \# r\left(S^{2} \times S^{2}\right)$ and let $e_{i}^{\prime}, f_{i}^{\prime}, 1 \leqslant i \leqslant r$, denote the classes in $\mathrm{H}_{2}(K ; \Lambda)$ represented by the $i$ th copies of $S^{2} \times\{\mathrm{pt}\}$ and $\{\mathrm{pt}\} \times S^{2}$, respectively. Let $e_{i}, f_{i}, 1 \leqslant i \leqslant r$ denote similar classes in $H_{2}\left(K \# r\left(S^{2} \times S^{2}\right)\right)$. Identify $K_{r}$ with $\left\langle e_{i}, f_{i}\right\rangle$ in the obvious fashion.

Then using Proposition 6.16 one can produce a self-diffeomorphism $\phi$ of $K \# r\left(S^{2} \times S^{2}\right)$ with $\phi_{*}\left(e_{i}^{\prime}\right)=\alpha\left(e_{i}\right)$ for $i=1, \ldots, r$. One now constructs a normal cobordism rel $\partial, F: W \rightarrow K \times I$ in two stages.

Firstly by doing surgery on $r$ trivial circles one can get a normal cobordism rel $\partial, \quad F_{1}: w_{1} \rightarrow K \times\left[0, \frac{1}{2}\right]$ with $\partial_{-} w_{1}=K, \partial_{+} w_{1}=K \# r\left(S^{2} \times S^{2}\right)$, $F_{1} \mid \partial_{-} w_{1}=\left(\mathrm{id}_{K}, 0\right)$ and $F_{1} \mid \partial_{+} w_{1}=\mathrm{id}_{K} \# r p, p: S^{2} \times S^{2} \rightarrow S^{2}$ the obvious natural projection map.

Let $S_{i}^{2} \times D^{2} \subset M \# r\left(S^{2} \times S^{2}\right) \# r\left(S^{2} \times S^{2}\right)$ be the standard embedding representing $e_{i}^{\prime}$. Using $\phi \mid S_{i}^{2} \times D^{2}$ we can perform framed surgery on $F_{1} \mid \partial_{+} w_{1}$ for $i=1, \ldots, r$ and obtain a normal cobordism $\left(F_{2}, B_{2}\right), F_{2}: w_{2} \rightarrow$ $K \times\left[\frac{1}{2}, 1\right]$ with $F_{2}\left|\partial_{-} w_{2}=F_{1}\right| \partial_{+} w_{1}, \partial_{+} w_{2}=K$ and $F_{2} \mid \partial_{+} w_{2}$ a homotopy equivalence. $\left(F_{2}, B_{2}\right)$ clearly has obstruction in $L_{5}(\pi, W)$ represented by $\alpha$. (See the argument in [Wa 3, Chapter 6].) Then letting $W=w_{1} \cup w_{2}$ and $F=F_{1} \cup F_{2}, B=B_{1} \cup B_{2}$ we obtain the desired normal cobordism $(F, W)$ with $F\left|W \rightarrow K \times[0,1], \partial_{-} W=\partial_{+} W=K, F\right| \partial_{-} W=\mathrm{id}_{K_{2}}$ and $\sigma(F, B)=\gamma$ as desired!

We now return to Theorem 6.17 and show it follows from Proposition 6.16. Thus let $\tau, \xi \in H_{2}(M, \Lambda)$ with $w_{2} H(\tau)=w_{2} H(\xi)=0$ and $\xi \cdot \tau=1$ and suppose $\mu(\xi)=\lambda \in \mathbf{Z}(\bmod I)$. Then by Proposition 6.16 (and use of the autodiffeomorphism of $S^{2} \times S^{2}$ which interchanges factors) we obtain autodiffeomorphisms $\phi, \psi$ of $M \# S^{2} \times S^{2}$ so that if $\gamma \equiv \mu(\tau)(\bmod I)$ then

(i) $\phi_{*}(e)=e+\xi-\lambda f ; \phi_{*}(f)=f ; \phi_{*}(\xi)=\xi-2 \lambda f ; \phi_{*}(\tau)=\tau-f$;

(ii) $\psi_{*}(e)=e ; \psi_{*}(f)=f+\tau-\gamma e ; \psi_{*}(\xi)=\xi-e ; \psi_{*}(\tau)=\tau-(\gamma+\bar{\gamma}) e$.

Now $\psi \phi(e+\lambda f)=\xi$ and $e+\lambda f$ is representable by an embedded sphere in $S^{2} \times S^{2}$ (recall $\lambda$ is an integer!), such that the inclusion of its complement in $M \#\left(S^{2} \times S^{2}\right)$ induces an isomorphism of fundamental groups! If $\lambda=0$ then $\xi$ is in addition representable by an embedded sphere with trivial normal bundle! Lastly to prove Proposition 6.16 one uses the same approach as in the proof of Proposition 6.8, that is one represents each $\omega \in H_{2}(M ; \Lambda)$ by means of an isotopy $h_{t}$ of some null homotopic $\phi\left(S^{1} \times 0\right), \phi \mid S^{1} \times D^{3} \rightarrow M$ an embedding, and obtains diffeomorphic copies $M_{i} ; i=0,1$ of $M \# S^{2} \times S^{2}$ via surgery on the different framings induced on tubular neighborhoods of $\phi\left(S^{1} \times 0\right)$ by $\phi\left(S^{1} \times D^{3}\right)$ and $h_{1} \phi\left(S^{1} \times D^{3}\right) . h_{1}$ induces a diffeomorphism $\hat{h}$ : $M_{0} \rightarrow M_{1}$ and essentially by direct computation one finds $\hat{h}_{*}$ to have the properties desired (modifying $\hat{h}_{*}$ if necessary by additional autodiffeomorphisms of $M \# S^{2} \times S^{2}=N \# S^{2} \times S^{2} \# S^{2} \times S^{2}$ induced by the known autodiffeomorphisms of $S^{2} \times S^{2} \# S^{2} \times S^{2}$ ). For full details see [CS 1].

As Cappell and Shaneson point out, in general one will not be able to realize all isometries of $\mathrm{H}_{2}\left(M \# S^{2} \times S^{2}\right)$ by means of diffeomorphisms. 
Consider the following example: Let $M=S^{1} \times S^{3}$. Then there exist by [Wa 3], $\alpha \in S U_{1}\left(\mathbf{Z}\left[\pi_{1}(M)\right]\right)$ representing the generator of $L_{5}\left(\pi_{1}(M)\right) \approx \mathbf{Z}$. Then there is no orientation preserving autodiffeomorphism of $M \# S^{2} \times S^{2}$ representing $\left(\mathrm{id}_{M}\right) \oplus \alpha$ on $H_{2}\left(M \# S^{2} \times S^{2} ; \mathbf{Z}(\mathbf{Z})\right)$ ! To see this suppose $\phi$ is such a diffeomorphism. Let $S^{2} \times D^{2}$ be a neighborhood of $S^{2} \times *$ in $M \# S^{2} \times S^{2}$ and use its image under $\phi$ to perform surgery on the normal map $(\pi, b): M \# S^{2} \times S^{2} \rightarrow M, b$ an appropriate bundle map covering the obvious projection $\pi$. What is obtained is a homotopy equivalence $h: Q \rightarrow S^{3}$ $\times S^{1}$ which is normally cobordant to the identity $I_{S^{3} \times S^{1}}$ via a normal cobordism $(W, B)$ with $\sigma(W, B)$ generating $L_{5}(\mathbf{Z})$. But this is impossible! For our construction shows that $Q$ is in fact diffeomorphic to $S^{3} \times S^{1}$ while the fact that $\sigma(W, B) \neq 0$ implies (see [HS], [Sh 2], [Sh 3]) that $Q \times S^{1}$ is not even PL-homeomorphic to $S^{3} \times T^{2}$ ! This is impossible!

We therefore see that a straightforward generalization of Theorem 6.7 is impossible. The best available generalization seems to be the following (for whose proof we refer the reader to [CS 1]):

THEOREM 6.7'. Suppose $M$ is a smooth compact connected four-manifold with $M=P \#\left(S^{2} \times S^{2}\right), N=M \#\left(S^{2} \times S^{2}\right)$ and set $\Lambda=\pi_{1}(M)$.

Suppose $H_{2}(M ; \Lambda)=L \oplus K_{0}$ is a direct sum decomposition of $\Lambda$-modules, orthogonal with respect to intersection, such that $K_{0}$ is a kernel with standard basis $e_{2}, \ldots, e_{r}, f_{2}, \ldots, f_{r}$. Let $e_{1}, f_{1}$ be the classes represented by the first and second spheres in the second summand of $M \# S^{2} \times S^{2}$ and write $H_{2}(N ; \Lambda)=$ $L \oplus K, K=\left\{e_{1}, f_{1}\right\} \oplus K_{0}$ where $\left\{e_{1}, f_{1}\right\}$ is a standard plane orthogonal to $K_{0}$.

Let $\alpha \in R L U_{r}(\Lambda)$, where $R L U_{r}(\Lambda)$ is as defined in [CS 1, Appendix]. Then there exists a diffeomorphism $\phi: N \rightarrow N$ inducing the identity on $\pi_{1}(N)$ so that, on $H_{2}(N, \Lambda), \phi_{*}=\left(\mathrm{id}_{L}\right) \oplus \alpha$.

6.5 Applications. Theorems 6.14 and 6.15 presently represent about all that is known about doing surgery in dimension 4 in the general case. The appropriate philosophy for dimension 4 thus seems to be "when in doubt, stabilize!"

We thus give some applications of the above theorems. We first note the following.

THEOREM 6.18 [CS 1]. Let $M=\mathbf{R} P^{4}$. Then for some $t>0$ there exists a smooth manifold $K$ that has the same (simple) homotopy type as $M \# t\left(S^{2} \times\right.$ $S^{2}$ ) but is not diffeomorphic or even smooth or PL normally cobordant to $M \# t\left(S^{2} \times S^{2}\right)$. In fact for all $r \geqslant 0, K \# r\left(S^{2} \times S^{2}\right)$ is not $h$-cobordant to $M \#(t+r)\left(S^{2} \times S^{2}\right)$. $K$ is unique up to $h$-cobordism and is topologically $h$-cobordant to $M \# t\left(S^{2} \times S^{2}\right)$. If $t$ is sufficiently large $K$ is diffeomorphic to $Q \# t\left(S^{2} \times S^{2}\right)$. ( $Q$ is the fake $\mathbf{R} P^{4}$ constructed in Chapter 4$)$.

Proof. Let $M=\mathbf{R} P^{4}$. Then using our information on $\pi_{*}(G / O)$ it is not difficult to show that $\mathcal{T}_{\mathrm{DIFF}}\left(\mathbf{R} P^{4}\right) \approx[M ; G / \mathrm{O}] \approx \mathbf{Z}_{4}$. Thus $\sigma:[M, G / \mathrm{O}] \rightarrow$ $L_{4}\left(Z_{2},-\right) \approx Z_{2}$ will be the natural nontrivial map.

Thus if $\theta \in[M, G / O]$ is the generator there exists a normal map $(f, b), f$ : $K_{0} \rightarrow M$ with $\sigma(f, b)=0$ but $\eta(f, b)=2 \theta \neq 0$. By the method used in the 
proof of Theorem 6.14 we obtain that for $r$ sufficiently large, $K=K_{0} \# r\left(S^{2}\right.$ $\times S^{2}$ ) has the homotopy type (in fact the simple homotopy type, since wh $\left(Z_{2}\right)=0$ ) of $M \# t\left(S^{2} \times S^{2}\right)$, some $t \geqslant 0$. Suppose $K$ were $h$-cobordant to $M \# t\left(S^{2} \times S^{2}\right)$. Then there would exist a normal map $(g, c) ; g$ : $M \# t\left(S^{2} \times S^{2}\right) \rightarrow M$ equivalent to $(f, b)$. Now since $\pi_{2} M=0$ we can do surgery to produce a homotopy equivalence $h: M \rightarrow M$ with $\eta(h)=\eta(g)=$ $\eta(f) \neq 0$. But as we pointed out in $\$ 4$ any self-homotopy equivalence $k$ of $\mathbf{R} P^{4}$ is homotopic to the identity and so satisfies $\eta(h)=0$. Thus $h$ can't exist. $K$ is unique up to $h$-cobordism since $L_{5}\left(Z_{2},-\right)=0 . K$ is however topologically $h$-cobordant to $M \# t\left(S^{2} \times S^{2}\right)$ since $[M, G / \mathrm{TOP}] \approx Z_{2} \oplus Z_{2}$ so $\eta_{\text {TOP }}(f, b)=0$. The topological surgery produces the $h$-cobordism. Lastly we wish to relate $K$ back to $Q$ of Chapter 4. To do this we must first analyze precisely why the map $f: Q \rightarrow \mathbf{R} P^{4}$ of that section was a homotopy equivalence not homotopic to the identity. Let us recall how $f$ was constructed.

(1) Given a matrix $A \in \mathrm{GL}(3, Z)$ satisfying $\operatorname{det} A=-1$ and $\operatorname{det}\left(I-A^{2}\right)=$ \pm 1 we let $\phi_{A}$ be the autodiffeomorphism induced by $A$ on $T^{3}$. We let $M_{A}$ be the mapping torus of $\phi_{A}$. Without loss of generality we may assume that for some 3-cell $e^{3} \subset T^{3}$ with $T_{0}^{3}=T^{3}-e_{3}, \phi_{A} \mid T_{0}^{3}$ maps $T_{0}^{3}$ into itself. Set $\phi_{0}=\phi_{A} \mid T_{0}^{3}$ and let $M_{0}=M_{0}(A)$ be the mapping torus of $\phi_{0}$. Note that $\partial M_{0}$ is the nonorientable orthogonal $S^{2}$-bundle $H$ over $S^{1}$ with corresponding nonorientable 3-disc-bundle $D(H)$ over $S^{1}$. Note that $M_{0} \cup_{H} D(H)$ is the mapping torus of $\phi_{A}$ and thus diffeomorphic to $M_{A}$.

(2) Let $N$ be the unique nonorientable orthogonal $S^{3}$-bundle over $S^{1}$. Then there exists a normal map of degree $1(h, b) ; h: M_{A} \rightarrow N, b: \nu_{M_{A}} \rightarrow \xi(\xi$ a bundle over $N$ ) with $h$ inducing isomorphisms on homology, commuting with projections to $S^{1}$ and covered by a map $\tilde{h}: \tilde{M}_{A} \rightarrow \tilde{N}$ (where ${ }^{\sim}$ means the orientable double covering space) also inducing isomorphisms on homology, such that $\eta(h, b) \in[N, G / \mathrm{O}]=\mathbf{Z}_{2}$ is nontrivial. Furthermore any map $h^{\prime}$ : $M_{A} \rightarrow N$ inducing isomorphism on homology will have $\eta\left(h^{\prime}\right) \neq 0$.

(Proof. Consider the composition $T^{3} \stackrel{\pi}{\rightarrow} S^{3} \stackrel{\beta}{\rightarrow} S^{3}$ where $T^{3} \stackrel{\pi}{\rightarrow} S^{3}$ has degree \pm 1 and $\beta$ is a reflection. Then $\beta \pi$ is homotopic to $\pi \phi_{A}$ and the homotopy induces the desired map $h$ between the mapping torus $M_{A}$ of $\phi_{A}$ and $N$ of $\beta$. To see $\eta(h) \neq 0$ let $\mathscr{F}$ be the 'exotic' framing on $T^{3}$ with $\mu\left(T^{3}, \mathscr{F}\right)=8$. Using $\mathscr{F}$ one constructs a bundle $\xi$ over $N$ and a map $b$ covering $h$. Then it can be shown that $\eta(h) \equiv 2 \mu\left(T^{3}, \mathscr{F}\right) / 16(\bmod 2) \equiv 1$ (mod 2) as desired. Using the covering homotopy property and the fact that $N$ is homologically an $S^{1}$ one obtains similarly that $\eta\left(h^{\prime}\right) \neq 0$.)

(3) There exists a homology equivalence $g: M_{0} \rightarrow D(H)$ extending the identity on the boundary and any such has $\eta(g) \neq 0$ in $[D(H) / H ; G / O] \approx$ $\mathbf{Z}_{2}$. Furthermore, any such homology equivalence is covered by a homology equivalence $\tilde{M}_{0} \rightarrow \widehat{D(H)}$.

(Proof. Using obstruction theory and the fact that $H_{*}(D(H))=H_{*}\left(S^{1}\right)$ we can extend the identity map on $H$ to the desired map $g$ covered by $\tilde{g}$ : $\tilde{M}_{0} \rightarrow D(H)$. To see that $\eta(g) \neq 0$, let $q: N \rightarrow N / D(H)=D(H) / H$ be the collapsing map and note that $q^{*}:[N / D(H) ; G / O] \rightarrow[N ; G / O]$ is an isomorphism. Furthermore $\eta(g)$ just goes to $\eta\left(g \cup_{H} \mathrm{id}_{D(H)}\right)=\eta(h) \neq 0$.) [Note that the natural map $[N ; G / \mathrm{O}] \rightarrow\left[N ; G /\right.$ TOP] is trivial so $\eta_{\text {TOP }}(g)=0$ in $[N ; G /$ TOP $]$. 
(4) Let $g:\left(M_{0}, H\right) \rightarrow(D(H), H)$ be as above. Let $Q=M_{0} \cup_{H} X_{1}$, where $X_{1}=\mathbf{R} P^{4}-D(H), D(H)$ realized as a tubular neighborhood of an orientation reversing element $y \in \pi_{1} X$ of order 2. Then $\mathbf{R} P^{4}=D(H) \cup_{H} X_{1}$ and so $f=g \cup_{H} \mathrm{id}_{X_{1}}$ is the desired map. By straightforward use of $1,2,3$ and some homotopy theory it can be checked that $f$ is a simple homotopy equivalence. We claim $\eta(f)$ is the unique nontrivial element in the kernel of $\left[R P^{4} ; G / O\right.$ ] $\rightarrow\left[\mathbf{R} P^{4} ; G / \mathrm{TOP}\right]$. Granting this, then the uniqueness of $K$ above shows that $K$ is $s$-cobordant to $Q \# t\left(S^{2} \times S^{2}\right)$ and thus if $t$ is sufficiently large is diffeomorphic to it. To prove the claim let $q: \mathbf{R} P^{4} \rightarrow D(H) / H$ be the collapsing map $\mathbf{R} P^{4} \rightarrow \mathbf{R} P^{4} /\left(\mathbf{R} P^{4}-D(H)\right) \rightarrow D(H) / H$. Then we obtain $\eta(f)$ as the image of $\eta(g)$ under $q^{*}[D(H) / H ; G / \mathrm{O}]=\mathbf{Z}_{2} \rightarrow\left[\mathbf{R} P^{4} ; G / \mathrm{O}\right]$. A straightforward calculation then shows that $q^{*}(1) \neq 0$. The fact that $\eta(f)$ lies in the kernel of $\left[\mathbf{R} P^{4} ; G / \mathrm{O}\right] \rightarrow\left[\mathbf{R} P^{4} ; G / \mathrm{TOP}\right]$ follows essentially from the remark after (3).

Now suppose $M=\mathbf{R} P^{2} \times S^{2}$, then one can show by a procedure entirely analogous to the proof of Theorem 6.18 that there exists a normal map $K_{0} \stackrel{f}{\rightarrow} M$ with $\sigma(f)=0$ and $\eta(f) \neq 0$ and there is by Theorem 6.14 therefore a simple homotopy equivalence $g: K \rightarrow M \# t\left(S^{2} \times S^{2}\right)=M_{t}, K=$ $K_{0} \# t\left(S^{2} \times S^{2}\right)$, with $\eta(g)=\eta(f) \neq 0$ and $\sigma(g)=0$. However the group $\operatorname{DIFF}\left(M_{t}\right)$ is not sufficiently well known to rule out the possibility that $g$ is in fact only an exotic auto equivalence (which was impossible for $M=\mathbf{R} P^{4}$ ). In fact the map $f: S^{2} \times \mathbf{R} P^{2} \rightarrow\left(S^{2} \times \mathbf{R} P^{2}\right) \bigvee S^{4} \stackrel{\text { id }}{\rightarrow}{ }^{2} S^{2} \times \mathbf{R} P^{2}$, where $\alpha$ is the generator of $\pi_{4}\left(S^{2}\right)$, is a homotopy equivalence not homotopic to a PL-homeomorphism because $\eta_{\mathrm{DIFF}}(f) \neq 0$ in $\left[S^{2} \times \mathbf{R} P^{2} ; G / \mathrm{PL}\right] . \eta_{\mathrm{TOP}}(f)=$ 0 in $\left[S^{2} \times \mathbf{R} P^{2} ; G /\right.$ TOP] and it is unknown whether $f$ is homotopic to a homeomorphism.

By a technique similar to the construction of $Q$ above one can produce a simple homotopy equivalence $Z \stackrel{h}{\rightarrow} S^{2} \times \mathbf{R} P^{2}$ between some manifold $Z$ and $S^{2} \times \mathbf{R} P^{2}$ with $\eta(h) \neq 0$. But again $Z$ may nevertheless be diffeomorphic to $S^{2} \times \mathbf{R} P^{2}$.

A similar situation occurs upon replacing $S^{2} \times \mathbf{R} P^{2}$ by $S^{3} \times S^{1}$. Applying Theorem 6.15 one can realize the generator of $L_{5}(Z)$ by a normal cobordism $(W, B)$ between $\operatorname{id}_{X} ; X=S^{3} \times S^{1} \# S^{2} \times S^{2}$, and some (simple) homotopy equivalence $f: M \rightarrow X$. Then using the argument of [Sh 1, §6, CS 1, §3] one obtains

Proposition 6.19 [CS 1]. Let $\tilde{\mathcal{S}}_{\mathrm{PL}}\left(\left(S^{2} \times S^{1}\right) \#\left(S^{3} \times S^{2}\right)\right)$ be Ker $\eta, \eta$ as in Theorem 6.3. Then there are precisely two elements in $\tilde{\mathcal{S}}_{\mathrm{PL}}\left(\left(S^{3} \times S^{1}\right) \#\left(S^{2}\right.\right.$ $\left.\times S^{2}\right)$ ).

Notes. 1. The existence of a homeomorphism representing the nontrivial element above can be shown to be equivalent to the existence of an almost parallelizable closed topological four-manifold of index 8 . In fact it can be shown that if any nonstandard 4-dimensional normal invariant is representable by a homeomorphism then such a manifold exists.

2. An explicit construction of the nontrivial element in $\tilde{\mathcal{S}}_{\mathrm{PL}}\left(\left(S^{3} \times\right.\right.$ $\left.S^{1}\right) \#\left(S^{2} \times S^{2}\right)$ ) can be found in [Sch 1]. The construction goes as follows: 
Let $P$ be the Poincaré homology sphere and suppose $\alpha \in \pi_{1}(P)$ is any noncentral element (there are $118 \mathrm{such}$ ).

Consider the natural embedding of $P$ in $S^{5}$ (i.e. $P=\left\{z_{1}^{2}+z_{2}^{3}+z_{3}^{5}=0\right\} \cap$ $\left.\left\{\left|z_{1}\right|^{2}+\left|z_{2}\right|^{2}+\left|z_{3}\right|^{2}=1\right\} \subset \mathbf{C}^{3}\left(z_{1}, z_{2}, z_{3}\right)\right)$. Now $S^{5}-P$ is a homology circle. Remove from $S^{5}$ a tubular neighborhood of $P$ and of a circle in $S^{5}-P$ generating $H_{1}\left(S^{5}-P\right) \approx \mathrm{Z}$. Call what is left $W$. An easy calculation shows that $W$ is a cobordism between $S^{3} \times S^{1}$ and $P \times S^{1}$ satisfying $H_{*}\left(W, \partial_{i} W\right)$ $=0$ and $P \times\{\mathrm{pt}\} \rightarrow \partial_{1} W \rightarrow W$ induces the trivial map $\pi_{1}(P) \rightarrow \pi_{1}(W)$. Now let $M$ be the manifold obtained by doing surgery on $\alpha$ in the normal homology equivalence $P \times S^{1} \rightarrow S^{3} \times S^{1}$.

It is easy to verify that this surgery then gives rise to a new PL cobordism $W^{\prime}$ between $\partial_{1} W=M$ and $\partial_{0} W=S^{3} \times S^{1} \# S^{2} \times S^{2}$ such that $W$ has trivial normal bundle and $H_{*}\left(W, \partial_{i} W ; \mathbf{Z}\right)=0$. Furthermore we can show that the identity map of $S^{3} \times S^{1} \# S^{2} \times S^{2}$ to itself extends to a normal degree 1 map $F: W \rightarrow\left(S^{3} \times S^{1} \# S^{2} \times S^{2}\right)$ with $f=F \mid \partial_{1} W: M \rightarrow S^{3} \times$ $S^{1} \# S^{2} \times S^{2}$ a homotopy equivalence. Then by an explicit calculation using Rohlin's theorem it can be shown that $f$ is not PL $s$-cobordant to the identity (what is demonstrated is that: $f$ being PL $s$-cobordant to the identity implies there exist a closed almost-parallelizable PL manifold of index 8. This is impossible by Rohlin's theorem. $f$ may however be TOP $s$-cobordant to the identity). Whether $M$ is itself PL homeomorphic (via some homeomorphism $g$ not homotopic to the identity) to $S^{3} \times S^{1} \# S^{2} \times S^{2}$ is not known. In [FP], Fintushel and Pao show that $M \# S^{2} \times S^{2}$ is diffeomorphic to $S^{3} \times$ $S^{1} \# 2\left(S^{2} \times S^{2}\right)$. Also Akbulut (Private Communication) has shown that $M \# P=S^{3} \times S^{1} \# 2 P \# Q$.

The techniques and consequences of Theorems 6.14 and 6.15 do not enable one to obtain direct nonstable information on 4-manifolds whose fundamental groups $\pi$ do not satisfy $H_{2}\left(\pi ; \mathbf{Z}_{2}\right)=0$. New techniques such as the homological surgery, Cappell and Shaneson used in constructing $Q$ seem to be necessary.

6.6 Some five-dimensional $h$-cobordisms. We note that all of the results in dimension 4 obtained above are relevant only to the question of determining a manifold $X$, or perhaps $X \# r\left(S^{2} \times S^{2}\right)$, up to $s$-cobordism or $h$-cobordism via calculation of normal invariants and surgery obstructions. Not having an $s$-cobordism theorem in dimension 4 we are still left with the problem of determining when two $s$-cobordant 4-manifolds are in fact diffeomorphic. No effective technique for doing this is known! Actually the techniques used in the proofs of Theorems 6.14 and 6.15 above can be used to prove (see also the work of Quinn [Q] to be described shortly) the generalization of Theorem 1.3 cited in Chapter 1 , that is, that $h$-cobordant 4-manifolds are stably diffeomorphic and that if the cobordism is an s-cobordism it is stably diffeomorphic to a product.

If the two ends of the $h$-coboraism are known a priori to be diffeomorphic then some partial results can be obtained. We thus present a Theorem (due to Barden [Bd] in the simply-connected case and Shaneson [Sh 1] in the $\pi_{1}=\mathbf{Z}$ case) giving what seems to be at present the most information we have on the structure of 5-dimensional $h$-cobordisms! 
THEOREM 6.20. Let $\left(W, V_{0}, V_{1}\right)$ be an h-cobordism with $W$ connected, $\operatorname{dim} W=5$ and $\pi_{1}(W)=\{e\}$ or $\pi_{1}(W)=\mathbf{Z}$ and $W$ orientable. Suppose $r$ : $W \rightarrow V_{0}$ is a retraction with $r_{1}=r \mid V_{1}$ a diffeomorphism.

Then there is a diffeomorphism $\phi: V \times I \rightarrow W$ with $\phi(x, 0)=x$ and $\phi(x, 1)$ $=r_{1}^{-1}(x)$ for $x \in V_{0}$.

Proof. Suppose firstly that $\pi_{1} W=\{1\}$. Let $\mu:\left(W, V_{0}, V_{1}\right) \rightarrow(I, 0,1)$ be a Morse function. Then $F=\left(r_{1}, \mu\right)$ is a homotopy equivalence and thus represents an element of $\delta(V \times I, V \times \partial I)$ (by hypothesis $F \mid V \times \partial I$ is a diffeomorphism). We must show that $F$ is the trivial element. Recall the exact sequence

$$
L_{6}(1) \stackrel{\omega}{\rightarrow} \delta_{5}^{\operatorname{DIFF}}(V \times I, V \times \partial I) \stackrel{\eta}{\rightarrow} \sigma_{5}^{\mathrm{DIFF}}(V \times I, V \times \partial I)
$$

of Theorem 5.9 .

We recall that $L_{6}\{1\}=P_{6}=Z_{2}$ and one can represent the nontrivial element of $L_{6}\{1\}$ by a degree 1 normal map $(\phi, B)$ with $\phi: S^{3} \times S^{3} \rightarrow S^{6}$ (see [Sh 1], [Sh 2]). Then the action of $\mathbf{Z}_{2}$ on $\mathcal{S}_{5}(V \times I, V \times \partial I)$ is defined by taking an element $h:(K, \partial K) \rightarrow(V \times I, V \times \partial I)$ in $\delta_{5}(V \times I, V \times \partial I)$ and forming the connected sum in the interior, $h \times 1 \# \phi:\left(K \times I \# S^{3} \times S^{3}\right)$ $\rightarrow V \times I \# S^{6}=V \times I$. Using the additivity of surgery obstructions (and the fact that $\sigma(h \times 1)=0)$ we see that $\sigma(h \times 1 \# \phi)=\sigma(\phi)$ so that the action of $L_{6}\{1\}$ on $\delta_{5}(V \times I, V \times \partial I)$ is trivial. Thus $\eta$ above is an injection and it suffices to prove that $\eta(F)=0$. We claim however that

$$
\mathscr{T}_{5}(V \times I, V \times \partial I) \approx \mathcal{T}_{5}\left(\Sigma V_{+}\right) \approx\left[\Sigma V_{+}, G / \mathrm{O}\right] \approx 0
$$

$\left(\Sigma V_{+}=V \times I / V \times \partial I\right)$. To see this note that $V$ being simply connected has a CW decomposition consisting of a zero cell, a 4-cell and some 2-cells. Thus $\Sigma V_{+}$has a corresponding decomposition consisting of a 1-cell, a 5-cell and some 3 cells. But as we mentioned previously $\pi_{i}(\mathrm{PL} / \mathrm{O})=0$ for $i<6$ and thus $\pi_{2 i+1}(G / \mathrm{O}) \approx \pi_{2 i+1}(G / \mathrm{PL})=0$ for $i<2$. In particular then by obstruction theory $\left[\Sigma V_{+}, G / \mathrm{O}\right]=0$. Thus $\eta(F)=0$, concluding the proof.

If $\pi_{1}(W)=\mathbf{Z}$ then using the fact [Sh 3] that $L_{6}(1) \rightarrow L_{6}(\mathbf{Z})$ is an isomorphism the proof of the injectivity of $\eta$ goes through unchanged. In this case however $\mathscr{T}_{5}^{\mathrm{DIFF}}(V \times I, V \times \partial I) \neq \varnothing$, so a different strategem must be used. What is done is to construct a homomorphism $\gamma: \mathscr{T}_{5}^{\mathrm{DIFF}}(V \times I, V \times \partial I) \rightarrow$ $\mathbf{Z}_{2}$ with the property that for $h:(M, \partial M) \rightarrow(V \times I, V \times \partial I), h$ a homotopy equivalence that is a diffeomorphism on the boundary, $\gamma(\eta(h))=0$ if and only if $\eta(h)=0$.

Next a homotopy equivalence $h: V \times I \rightarrow V \times I$ is constructed with $h \mid V \times \partial I$ the identity and $\eta(h) \neq 0$. ( $h$ is constructed by showing that (1) a generator of $H_{3}(V)=\mathrm{Z}$ is spherical, (2) letting $\alpha_{0} \in \pi_{5}(V \times I)$ be the element obtained by composing the nontrivial map $S^{5} \stackrel{P}{\rightarrow} S^{3}$ with the map $S^{3} \stackrel{\beta}{\rightarrow} V \times\left(\frac{1}{4}, \frac{3}{4}\right)$ representing the generator of $H_{3}(V)$, (3) defining $h: V \times I$ $\rightarrow V \times I$ as the identity outside some disc $D^{5} \subset V \times\left(\frac{1}{4}, \frac{3}{4}\right)$ and so that the obstruction to homotoping $h$ to the identity $\operatorname{rel}(V \times I-D)$ is precisely $\alpha_{0} \in H^{5}\left(V \times I,(V \times I-D), \pi_{5} V\right) \approx \pi_{5} V,(4)$ using a 'Thom construction' argument to show that $\eta(h) \neq 0$. (See p. 350 of [Sh 1] for details.) 
Lastly if $\eta(F) \neq 0$ then letting $\phi=h \circ F$ we see that $\gamma(\eta(\phi))=0$ so $\eta(\phi)=0$, and thus $\phi$ is the desired diffeomorphism.

6.7 Stable surgery and the Whitney trick. As we have mentioned repeatedly, the major difficulties in the classification of 4-manifolds arise as a result of the failure of Whitney's lemma in dimension 4. We have exhibited methods of getting around this failure by using a knowledge of the diffeomorphisms of $M \# r\left(S^{2} \times S^{2}\right)$ as well as the existence of embedded spheres representing the homology class $e+\lambda f\left(e, f\right.$ as in Theorem 6.16) in $H_{2}\left(M \# r\left(S^{2} \times S^{2}\right)\right)$ to represent other classes $\xi$ by embedded spheres and thus do stable surgery. The problem with this approach is that it is usually quite difficult to understand $\operatorname{DIFF}(X)$ for a 4-manifold $X$ and for the purposes of doing surgery it is generally not necessary to have a complete picture of all of $\operatorname{DIFF}(X)$. For example in the proof of Theorems 6.6 and 6.7 we made use of various algebraic information (such as Proposition 6.8) the proof of which is far from trivial. We would therefore like to sketch different approaches to stable surgery due to Lawson [Law 1], [Law 2], [Law 3], Quinn [Q] and Casson [Cas 1] and based on a cute trick of Norman's [Nor] to circumvent the failure of Whitney's lemma in dimension 4.
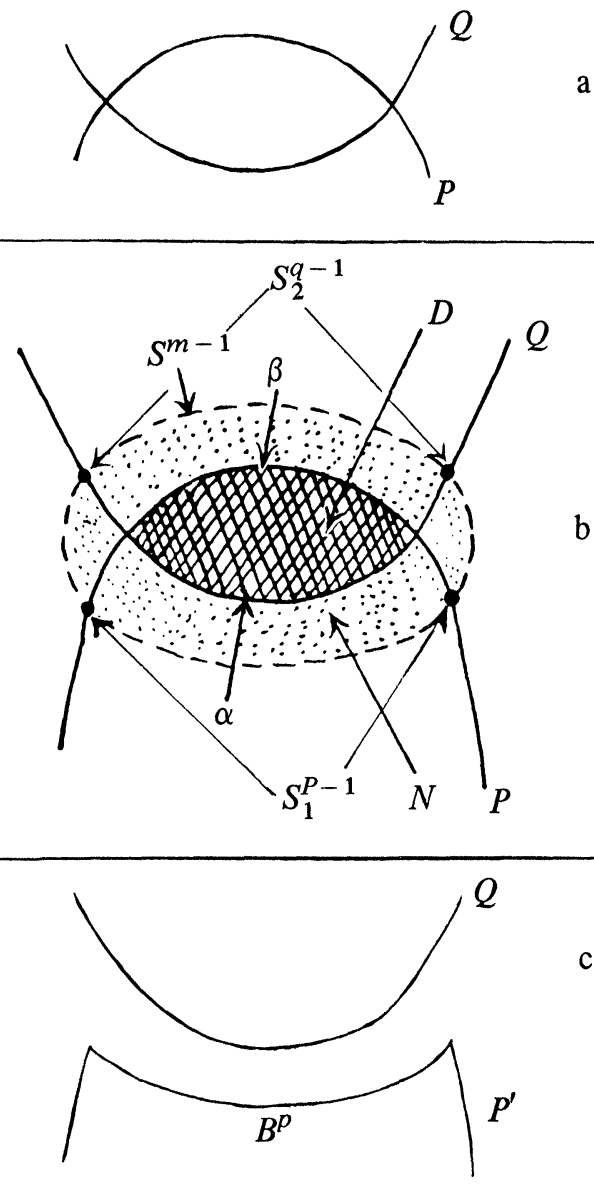

FIGURE 6.2 
Let us return to Whitney's lemma as stated in Chapter 1 .

Thus let $P^{p}, Q^{q}$ be submanifolds of $M^{m}$ with $p+q=m$ and suppose $p, q \in P \cap Q$ are transversal intersection points with $(P \cdot Q)_{p}=-(P \cdot Q)_{q}$. Let $\alpha, \beta$ be paths in $P$, resp. $Q$, connecting $p$ with $q$ so that $\alpha \cup \beta$ is an oriented circle. Then the proof of Whitney's lemma runs as follows ([RS], [Whit 1]). (See Figure 6.2.)

(1) Let $D$ be an embedded 2-disc in $M$ with $\partial D=\alpha \cup \beta=\gamma$ and $D \cap(P$ $\cup Q)=\partial D^{2}$. ( $D$ is called a Whitney disc. It exists in sufficiently high dimensions by a general position argument. See [RS, p. 71].) In dimension $m=4$ we can only find an immersed disc $f: D^{2} \rightarrow M$ with $\partial f\left(D^{2}\right)=\alpha \cup \beta$ and $D \cap(P \cup Q)=\partial D^{2} \cup\left\{\right.$ transversal intersection points $x_{1}, \ldots, x_{r}$, for some $r \geqslant 0\}$.

(2) Let $\left(N, B_{1}, B_{2}\right)$ be a regular neighborhood of $D^{2}$ in $(M, P, Q)$. Then it is easily seen that $\left(N, B_{i}\right)$ is an unknotted ball pair $(i=1,2)$ and that $\left(B_{1} \cdot B_{2}\right)$ (keeping boundaries fixed) is just zero. Then letting $\left(S^{m-1}, S_{1}^{p-1}, S_{2}^{q-1}\right)=\partial\left(N, B_{1}, B_{2}\right)$ it can be shown (if $\left.m \geqslant 4\right)$ that $S_{1}^{p-1}$ is unlinked from $S_{2}^{q-1}$ in $S^{m-1}$.

(3) Then there exists a ball $B^{p} \subset S^{m-1}$ with $\partial B^{p}=S_{1}^{p-1}$ and $B^{p} \cap B_{2}=$ $\varnothing$ and $B^{p}$ can be used to isotope $P$ to $P^{\prime}$ with $P^{\prime} \cap Q=P \cap Q-\{p, q\}$.

Now for $m=4$, Step (2) above works but there is the problem of the self-intersections of $D$ and its intersection with $P \cup Q$. Norman's trick is then based on the following lemma.

Lemma 6.21. Suppose $M$ is a smooth connected closed 4-manifold and let $f_{i}$ : $N_{i}^{2} \rightarrow M$ be proper $n_{i}$-point immersions with $f_{i} \mid \partial N_{i}$ an embedding, $i=1,2$. ( $A$ proper map $f: N \rightarrow M$ is one which satisfies $f^{-1}(\partial M)=\partial N$ and an n-point immersion is one which has $n$-distinct transverse self-intersections.) Suppose there exists an embedded 2-sphere $S^{2}$ in $\operatorname{int}(M)$ which intersects $f\left(N_{1}\right)$ transversely in a single point $x$.

Then: (1) There exists a proper $\left(n_{1}-1\right)$-point immersion $\tilde{f}_{1}: N_{1} \rightarrow M$ with $\tilde{f}_{1}\left|\partial N_{1}=f_{1}\right| \partial N_{1}$.

(2) If $S^{2}$ has trivial normal bundle there exists a proper embedding $\bar{f}_{1}$ : $N_{1} \rightarrow M$, with $\bar{f}_{1}\left(N_{1}\right)$ homologous to $f_{1}\left(N_{1}\right)$ and $\bar{f}_{1}\left|\partial N_{1}=f\right| \partial N_{1}$. If $M$ is 1-connected $\bar{f}_{1}$ can be chosen homotopic to $f_{1}$.

(3) If in (2) above $S^{2}$ is disjoint from $f_{2}\left(N_{2}\right)$ then there exists a proper $n_{2}$-point immersion $\bar{f}_{2}: N_{2} \rightarrow M-S^{2}$ with $\bar{f}_{2}\left(N_{2}\right) \cap \bar{f}_{1}\left(N_{1}\right)=\varnothing, \bar{f}_{2}\left|\partial N_{2}=f_{2}\right| \partial N_{2}$ and $\left[\bar{f}\left(N_{2}\right)\right]$ homologous to $\left[f_{2}\left(N_{2}\right)\right]+r\left[S^{2}\right]$ for some $r \in \mathbf{Z}$.

Proof. (See Figure 6.3.) Let $p$ be a self-intersection point of $f_{1}\left(N_{1}\right)$ and let $\Delta_{1}$ be an open disc in one sheet of $f_{1}\left(N_{1}\right)$ about $p$ and $\Delta_{2}$ an open disc in $S^{2}$ about $x$. Let $\alpha$ be a path in $f_{1}\left(N_{1}\right)$ connecting $p$ and $x$ (starting in the sheet not containing $\left.\Delta_{1}\right)$. We can pipe $f_{1}\left(N_{1}\right)-\Delta_{1}$ together with $S^{2}-\Delta_{2}$ along $\alpha$ to obtain the requisite $\tilde{f}_{1}$. If $S^{2}$ has trivial normal bundle we can take $n$-disjoint cross sections to eliminate all self-intersections of $f_{1}$ and get an embedding $\hat{f}_{1}$. Homologically at each stage we are forming a sum $f_{1}\left[N_{1}\right] \pm\left[S^{2}\right]$ and if we have the embedding $\hat{f}_{1}$ satisfying $\left[\hat{f}_{1}\left(N_{1}\right)\right]=\left[f_{1}\left(N_{1}\right)\right]+r\left[S^{2}\right]$ we can use the fact that $\hat{f}_{1}\left(N_{1}\right)$ still intersects $S^{2}$ transversely to obtain a new embedding $\tilde{f}_{1}$ : $N_{1} \rightarrow M$ with $\left[\tilde{f}\left(N_{1}\right)\right]=\left[\hat{f}_{1}\left(N_{1}\right)\right]-r\left[S^{2}\right]=\left[f_{1}\left(N_{1}\right)\right]$ leaving $\partial N_{1}$ unchanged. Lastly if $M$ is 1-connected we use Hurewicz's theorem to change a homology 
to a homotopy. To prove (3) we form connected sums $f_{2}\left(N_{2}\right) \# r\left[S^{2}\right]$ exactly as above to make it disjoint from $f_{1}\left(N_{1}\right)$.
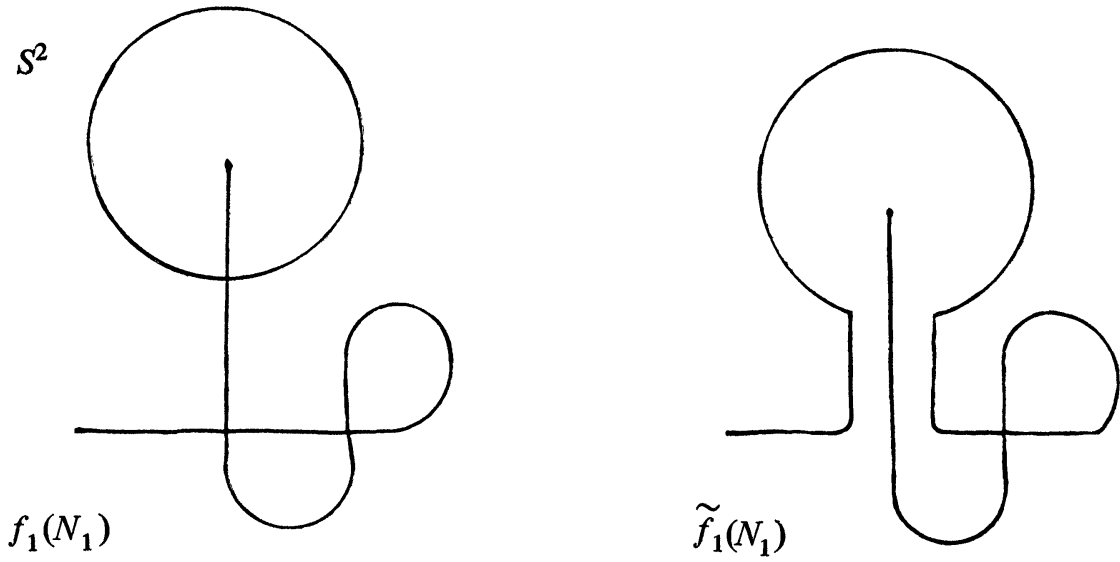

Figure 6.3

If $\alpha: N \rightarrow M$ is a proper immersion we shall say it has a complementary 2-sphere if there exists a 2-sphere $S^{2} \subset \operatorname{int}(M)$ intersecting $\alpha(N)$ transversely in one point. $S^{2}$ will be called a strong complement if in addition it has trivial normal bundle. (More generally we shall say the $\alpha_{i}: N_{i} \rightarrow M$ are complementary if they intersect transversely in one point in $\operatorname{int}(M)$.) Note that if $\alpha$ : $N \rightarrow M_{0}$ is a proper $n$-point immersion, where $M_{0}=M-\{4$-disc $\}$, then there clearly always exists an immersion $\bar{\alpha}: N \rightarrow M \# S^{2} \times S^{2}$ with $i \circ \alpha \mid \partial N$ $=\bar{\alpha} \mid \partial N$ and $\bar{\alpha}: N \rightarrow M$ having a strongly complementary 2-sphere (where $i$ : $M_{0} \rightarrow M \# S^{2} \times S^{2}$ is the obvious embedding).

Using the methods of Lemma 6.21, Quinn [Q] and Lawson [Law 1], [Law 2], [Law 3] prove the following.

Theorem 6.22 (STABle $s$-COBORdism, COMPare Theorem 1.7). Suppose $\left(W^{5}, M_{+}, M_{-}\right)$is a PL $s$-cobordism with nroduct structure on the boundary cobordism from $\partial M_{+}$to $\partial M_{-}$. Let $J$ be an arc from $M_{+}$to $M_{-}$. Then for some $k$ the product structure on the boundary extends to $\left(W \# k\left(S^{2} \times S^{2} \times I\right)\right.$, $\left.M_{+} \# k\left(S^{2} \times S^{2} \times\{0\}\right), M_{-} \# k\left(S^{2} \times S^{2} \times\{1\}\right)\right)$.

(Recall that as in Chapter 1 we can obtain the diffeomorphism on the ends with only the assumption that the cobordism be an $h$-cobordism.)

TheOREM 6.23 (SpeCiAl HANDLEBOdY-DeCOMPOSITION). Suppose $0 \neq N^{3} \subset$ $\partial M^{4}$ is a submanifold and $(M, N)$ is 1-connected. Then for some $k$, $\left(M \#_{\partial} k\left(D^{2} \times S^{2}\right), N \# k\left(S^{1} \times S^{2}\right)\right)$ has a handle decomposition with no 1-handles.

(Theorem 6.23 is false without stabilization if $\partial M \neq \varnothing$. See our description of Casson's counterexample in Chapter 4.)

In addition one could prove analogues of the Cappell and Shaneson stable surgery results, Theorems 6.14 and 6.15 using the above approach. We shall content ourselves with describing an alternative proof of Lemma 6.13 due to Casson which does not depend on Theorem 6.7. 
The basic idea behind the proofs of Theorems 6.22 and 6.23 is to use Lemma 6.21 to replace the Whitney lemma by the following

Lemma 6.24 (Stable Whitney LeMma). Suppose $\alpha: P^{2} \rightarrow M^{4}, \beta: Q^{2} \rightarrow M^{4}$ are proper $n_{i}$-point immersions with disjoint strongly complementary 2-spheres $S_{P}, S_{Q}$ such that $S_{P} \cap \beta(Q)=\alpha(P) \cap S_{Q}=\varnothing$. Suppose $\alpha(P)$ intersects $\beta(Q)$ transversely with $p, q \in \alpha(P) \cap \beta(Q)$ having algebraic intersection $(\alpha(P), \beta(Q))_{p}=-(\alpha(P), \beta(Q))_{q}$. Then there exists proper $n_{i}$-point immersions $\alpha^{\prime}: P^{2} \rightarrow M^{4} \# S^{2} \times S^{2}, \beta^{\prime}: Q \rightarrow M^{4} \# S^{2} \times S^{2}$ with strongly complementary 2-spheres such that if $i: M_{0} \rightarrow M^{4} \# S^{2} \times S^{2}$ is the obvious embedding then $\alpha^{\prime}\left(P^{2}\right)$ intersects $\beta^{\prime}(Q)$ transversely, $\alpha^{\prime}\left(P^{2}\right) \cap \beta^{\prime}\left(Q^{2}\right)=i \alpha\left(P^{2}\right) \cap i \beta\left(Q^{2}\right)$ $-\{p\}-\{q\}$ and $\alpha^{\prime}\left(P^{2}\right)$ regular homotopic (isotopic if $n_{1}=0$ ) to i $\alpha\left(P^{2}\right)$, $i$ as above.

Proof. Let $\gamma$ be the circuit $\gamma=\alpha \cup \beta$ constructed in the proof of the Whitney lemma above and suppose $D=\eta\left(D^{2}\right)$ is an immersed disc with $D \cap(P \cup Q)=\partial D^{2} \cup\{$ transversal intersection points $\}$.

Using the note preceding Theorem 6.22 , if we extend our maps $\alpha, \beta, \eta$ to $\alpha, \beta, \eta$ into $M \# S^{2} \times S^{2}$ then $i(D)$ has a strongly complementary 2 -sphere $S_{D}$ which is disjoint from $\alpha(P), \beta(Q), i\left(S_{P}\right), i\left(S_{Q}\right)$. Using $S_{D}$ we can make $\eta$ into an embedding and using $i\left(S_{P}\right), i\left(S_{Q}\right)$ we can make $\eta\left(D^{2}\right)$ disjoint from $\alpha(P) \cup \beta(Q)$. Then we can continue the proof of the Whitney lemma so as to get the desired result.

Then the proof of Theorem 6.22 can be obtained by imitating the proof of the $s$-cobordism theorem in higher dimensions until the use of the Whitney lemma is needed. At that point Lemma 6.24 and the following Lemma 6.25 whose proof we leave to the reader (see [Q]) and [Law 1], [Law 2], [Law 3] suffice to complete the proof.

LEMMA 6.25. Let $M^{4}$ be a closed 4-manifold and suppose $\alpha_{i}: S^{2} \rightarrow M^{4}$ are either proper $n_{i}$-point immersions or embeddings, $i=1, \ldots, N$, and there exist $\xi_{i} \in H_{2}\left(M, Z_{1}(M)\right)=\pi_{2}(M)$ with $w_{2} H\left(\xi_{i}\right)=0$ such that $\left[\alpha_{i}\left(S^{2}\right)\right] \cdot \xi_{j}=\delta_{i j}$ and in the embedding case $\pi_{1}\left(M-\alpha_{i} S^{2}\right) \rightarrow \pi_{1} M$ is an isomorphism for $i=$ $1, \ldots, N$.

Then: (1) There exist proper $n_{i}$-point immersions $\hat{\alpha}_{i}: S^{2} \rightarrow M^{4} \# k\left(S^{2} \times\right.$ $\left.S^{2}\right)$ for some $k>0, i=1, \ldots, N$, such that (i) each $\hat{\alpha}_{i}$ has a strongly complementary 2-sphere $S_{i} \subset M^{4} \# k\left(S^{2} \times S^{2}\right)$ with all the $S_{i}$ pairwise disjoint and $S_{i} \cap \hat{\alpha}_{j}\left(S^{2}\right)=\varnothing, i \neq j$, and (ii) the $\hat{\alpha}_{i}$ are regularly homotopic (isotopic in the embedding case) to i $\alpha_{i}: S^{2} \rightarrow M^{4} \# k\left(S^{2} \times S^{2}\right), i: M-d^{4} \rightarrow$ $M^{4} \# k\left(S^{2} \times S^{2}\right)$ the obvious map.

(2) Suppose $\alpha_{i}$ above extends to $\alpha_{i}: S^{2} \times D^{2} \rightarrow M^{4}$ and $\lambda\left(\alpha_{i}, \alpha_{j}\right)=\mu\left(\alpha_{i}\right)=0$ all $1 \leqslant i, j \leqslant N$ where $\lambda, \mu$ are the intersection and self-intersection forms defined as in [Wa 3]. Then the $\hat{\alpha}_{i}$ can be taken to be disjoint embeddings. $\hat{\alpha}_{i}$ : $S^{2} \times D^{2} \rightarrow M^{4} \# k\left(S^{2} \times S^{2}\right)$.

(3) Furthermore if in (2) above there exists an embedding $\beta: S^{2} \rightarrow M^{4}$ with strongly complementary 2-sphere such that $\left[\beta\left(S^{2}\right)\right] \cdot\left[\alpha_{1}\left(S^{2}\right)\right]= \pm 1$ and $\left[\beta\left(S^{2}\right)\right] \cdot\left[\alpha_{i}\left(S^{2}\right)\right]=0$ for $i>1$ then there is an isotopy in $M \# k\left(S^{2} \times S^{2}\right)$ of $\beta$ to $\hat{\beta}$ such that $\hat{\beta}\left(S^{2}\right)$ is disjoint from $\hat{\alpha}_{i}\left(S^{2}\right)$ for $i>1$ and intersects $\hat{\alpha}_{1}\left(S^{2}\right)$ transversely in one point. 
(With reference to Lemma 6.25 we note only that to carry out the 'algebraic complement implies stable geometric complement' part of the proof of Theorem 6.22 we need to know that boundary spheres $S$ in the middle level of our cobordism satisfy $\pi_{1}(M-S) \approx \pi_{1}(M)$ to apply part 1 of the lemma. This follows immediately from the fact that such boundary spheres have strongly complementary 2-spheres $S^{\prime}$ in $M \# k\left(S^{2} \times S^{2}\right)$.)

Theorem 6.23 can also be proven using Lemmas 6.24 and 6.25, though there are some additional technical details for which we refer to [Q] and [Law 1], [Law 2], [Law 3].

We conclude this section with a direct proof of Lemma 6.13 (and thereby complete the proof of Theorem 1.2) due to Casson based on the above type of reasoning. In particular noting the way we proved Lemma 6.13 it suffices to prove

THEOREM 6.26. Let $M$ be a connected sum of copies of $S^{2} \times S^{2}$ and $S^{2} \times S^{2}$. Then any isometry of $\left(H_{2}(M), L_{M}\right)$ is induced by a diffeomorphism.

The proof of Theorem 6.26 is based on the following two lemmas whose straightforward proofs we omit.

Lemma 6.27. Suppose $M$ is a 1-connected 4-manifold and $S \subset M$ is an embedded $S^{2}$ with $[S]^{2}=0$ and $S^{\prime}, S^{\prime \prime}$ 2-spheres complementary to $S$ with $\left[S^{\prime}\right]^{2}=\left[S^{\prime \prime}\right]^{2}$. Then there exists an autodiffeomorphism $g$ of $M$ with $g\left(S^{\prime}\right)=S^{\prime \prime}$ and $g(S)=S$.

LEMMA 6.28. Let $M$ be a 1-connected 4-manifold and suppose $x$ is a primitive ordinary element of $H_{2}(M)$ with $x^{2}=0$. Suppose $M=N \# E$ for some 4-manifold $N$ and $E$ an $S^{2}$-bundle over $S^{2}$. Let $S$ be the class in $H_{2}(M)$ of a fiber of $E$. Then there exists an autodiffeomorphism $f$ of $M$ with $f_{*}(S)=x$.

Proof of THEOREM 6.26. Suppose $M=E_{1} \# \ldots \# E_{k}, E_{i}$ an $S^{2}$ bundle over $S^{2}$ where we assume without loss of generality that $E_{i}, i \leqslant t$, is the nontrivial bundle and $E_{i}, i>t$, the trivial one.

(1) If $k=1$ a direct construction demonstrates the truth of the theorem.

(2) If $k>1$ let $S_{i}$ be the class of the fiber of $E_{i}$ in $H_{2}(M)$ and note that $S_{i}$ is a primitive ordinary class. Thus if $x \in H_{2}(M)$ is primitive and ordinary with $x^{2}=0$ there is by Lemma 6.28 an element $g \in \operatorname{DIFF}(M)$ with $g\left(S_{k}\right)=x$. Suppose $\phi$ is an isometry of $H_{2}(M)$. Then taking $x=\phi\left(S_{k}\right)$ we see $g_{*}\left(S_{k}\right)=$ $\phi\left(S_{k}\right)$. Let $S_{k}^{\prime}$ be the class in $H_{2}(M)$ of a cross section of $E_{k}$. Then $\left[g_{*}\left(S_{k}^{\prime}\right)\right]^{2}=$ $\left[\phi\left(S_{k}^{\prime}\right)\right]^{2}$ and both $g_{*}\left(S_{k}^{\prime}\right)$ and $\phi\left(S_{k}^{\prime}\right)$ are complementary to $x$. Thus by Lemma 6.25 we may assume without loss of generality that $g\left(S_{k}^{\prime}\right)=\phi\left(S_{k}^{\prime}\right)$. Thus $g_{*}^{-1} \phi \mid H_{2}\left(\#_{i=1}^{k-1} E_{i}\right)$ and $g_{*}^{-1} \phi \mid H_{2}\left(E_{k}\right)$ are isometries and our proof concludes by induction.

6.8 Flexible handles. Casson [Cas 2], [Cas 3] has devised a different approach to possibly getting around the 4-dimensional difficulties inherent in the Whitney lemma. In essence his idea is to repeatedly attempt to replace cancelling pairs of points by means of Whitney discs even though intersections are introduced. With care though all such intersections can be 'banished to $\infty$ ' and results up to proper homotopy obtained. More precisely we have: 
THEOREM 6.29 (CASSON). Suppose $W^{4}$ is a simply-connected smooth 4-manifold and $\alpha, \beta \in H_{2}(W ; Z)$ with $\alpha^{2}=\beta^{2}=0$ and $\alpha \cdot \beta=1$. Then there exists an open set $V \subset W$ with the proper homotopy type of $S^{2} \times S^{2}-\{\mathrm{pt}\}$ such that $\alpha, \beta \in \operatorname{Im}\left(H_{2}(V)\right)$.

If $V$ above were, in fact, diffeomorphic to $S^{2} \times S^{2}-\{\mathrm{pt}\}$ then one could do 4-dimensional surgery without further restrictions. In particular, many stable 4-dimensional results could be replaced by corresponding absolute results. For example smooth manifolds $M$ with intersection form $L_{M}=E_{8} \oplus$ $E_{8}$ or $L_{M}=E_{16}$ would exist.

To prove Theorem 6.29 we begin by introducing some notation. Thus suppose $W$ is an oriented 4-manifold, not necessarily compact. The element of $H_{2}(W ; \partial W)$ represented by a map $f: D^{2} \rightarrow W$ carrying $S^{1}$ into $\partial W$ will be written $[f]$. Maps $f, g: D^{2} \rightarrow W$ carrying $S^{1}$ to disjoint subsets of $W$ have an intersection number $f \cdot g$. This is invariant under homotopy (rel $S^{1}$ ), and may be obtained by counting the intersections (with signs) of transverse approximations to $f$ and $g$. We can then state:

LEMMA 6.30 (CASSON). Let $W^{4}$ be a smooth simply-connected manifold and suppose $f_{i}: D^{2} \rightarrow W^{4}, i=1, \ldots, n$, are proper maps with

(i) $f_{i} \mid \partial D^{2} \rightarrow \partial W$ disjoint embeddings,

(ii) $\left[f_{i}\right] \cdot\left[f_{j}\right]=0$ for all $i \neq j$,

(iii) There exist $\alpha_{i} \in H_{2}(W)$ with $\alpha_{i}^{2}$ even and $\alpha_{i} \cdot\left[f_{j}\right]=\delta_{i j}$.

Then there exist disjoint open sets $V_{i} \subset W$ such that:

(1) $\left(V_{i}, V_{i} \cap \partial W\right)$ has the proper homotopy type of $\left(D^{2} \times \mathbf{R}^{2}, S^{1} \times \mathbf{R}^{2}\right)$,

(2) $V_{i} \cap \partial W$ is an open regular neighborhood of $f_{i}\left(S^{1}\right)$,

(3) $f_{i}$ is homotopic (rel $S^{1}$ ) to a map into $V_{i}$.

The $V_{i}$ are called flexible handles. If they were in fact diffeomorphic to honest 2-handles then $W$ in Theorem 6.29 would in turn also be diffeomorphic to $S^{2} \times S^{2}-\{\mathrm{pt}\}$.

Proof of Theorem 6.29. Let $\alpha, \beta$ be as in Theorem 6.29. Using the Hurewicz theorem and the fact that $\alpha^{2}=\beta^{2}=0$ we can find immersions $\tilde{f}_{i}$ : $S^{2} \times R^{2} \rightarrow W, i=1,2$ representing $\alpha$ and $\beta$ such that $\tilde{f}_{1}\left(S^{2} \times 0\right)$ intersects $\tilde{f}_{2}\left(S^{2} \times 0\right)$ transversely. Let $x$ be a point of positive intersection and let $B$ be an open 4-ball about $x$ so that $f_{i}=\tilde{f}_{i} \mid f^{-1}(\overline{W-B})$ satisfies (i) $\left[f_{1}\right] \cdot\left[f_{2}\right]=0$, (ii) $f_{i}$ is an immersion of $D^{2} \times \mathbf{R}^{2}$ into $X=\overline{W-B}$ with $f_{i} \mid S^{1} \times \mathbf{R}^{2}$ an embedding. Note that $\alpha, \beta$ pull back to classes $\tilde{\alpha}, \tilde{\beta}$ in $H_{2}(X)$ which satisfy $\tilde{\alpha} \cdot\left[f_{1}\right]=\tilde{\beta} \cdot\left[f_{2}\right]=0, \tilde{\alpha} \cdot\left[f_{2}\right]=\tilde{\beta} \cdot\left[f_{1}\right]=1$ and $\tilde{\alpha}^{2}=\tilde{\beta}^{2}=0$. Then applying Lemma 6.30 we can find $V_{1}, V_{2}$ as in that lemma. Setting $V=B \cup V_{1} \cup V_{2}$ - \{any boundary we see that $V$ has the desired properties.

Before discussing the proof of Lemma 6.30 we give an additional application.

Suppose $W$ is an open manifold. Then

(1) $U$ is a neighborhood of $\infty$ in $W$ if $\overline{W-U}$ is compact.

(2) $W$ is $r$-connected at $\infty$ if every neighborhood $U$ of $\infty$ contains a neighborhood $V$ of $\infty$ such that every map $K^{r} \rightarrow V\left(K^{r}\right.$ an $r$-complex $)$ is null homotopic in $U$.

Then in dimension $n \neq 1,3,4$ we have the Browder-Levine-Livesay-Wall theorem which says: 
THEOREM 6.31 [BLL]. Let $W^{n}$ be a PL-manifold with compact boundary, $n \neq 1,3,4$. Then $W \cup\{\infty\}$ (the one-point compactification of $W$ ) is a PLmanifold if and only if $H_{*}(W)$ is finitely generated and $W$ is $[(n-1) / 2]-$ connected at $\infty$.

As a consequence of Lemma 6.30 we can now prove:

THEOREM 6.32 (CASSON). There exists a smooth open 4-manifold $W$ with the proper homotopy type of either $S^{2} \times S^{2}-\{\mathrm{pt}\}$ or $S^{3} \times R$, but not diffeomorphic to any closed manifold - $\{\mathrm{pts}\}$ (i.e. the ends of $W$ don't have neighborhoods of the form $S^{3} \times R$ ).

Proof. Let $P$ be the Poincaré homology sphere and consider $Y=P \times I$. Let $\alpha$ be a noncentral element of $\pi_{1}(Y)$ (there are 118 such) and surger $Y$ along a smooth $S^{1}$ in $\operatorname{int}(Y)$ representing $\alpha$. Call the resultant manifold $X$ and note that $\pi_{1}(X)=1$ and $\partial X=P \cup(-P)$.

Then using the exact sequence associated to the surgery it is easy to check that $H_{*}(X)=H_{*}\left(S^{2} \times S^{2}-2\right.$ \{pts $\left.\}\right)$. In particular there exists $\alpha, \beta \in$ $H_{2}(X ; \mathbf{Z})$ with $\alpha^{2}=\beta^{2}=0$ and $\alpha \cdot \beta=1$ and so by Theorem 6.29 there exists an open set $V \subset X$ as in that theorem with $\alpha, \beta \in \operatorname{Im}\left(H_{2}(V)\right)$ and $V$ proper homotopy equivalent to $S^{2} \times S^{2}-\{\mathrm{pt}\}$. Then (1) either $V$ is not diffeomorphic to a closed manifold $-\{p t\}$ and we are finished or (2) $V$ is diffeomorphic to some $M-\{\mathrm{pt}\}$. In case (2) the ends of $V$ have a neighborhood diffeomorphic to $S^{3} \times R$.

We can then decompose $X$ as an interior connected sum $Z \# S^{2} \times S^{2}$ where $Z$ is a manifold with $\partial Z=P \cup(-P)$ and $H_{*}(Z)=H_{*}\left(S^{3}\right)$. Joining the ends of $Z$ together we get a manifold homotopy equivalent to $S^{3} \times S^{1}$ and letting $W$ be its universal cover we find that $W$ is proper homotopy equivalent to $S^{3} \times R$. However neither end of $W$ has a neighborhood diffeomorphic to $S^{3} \times R$. For suppose one end had such a neighborhood. Then attaching a $B^{4}$ in the obvious fashion we see that there exists an embedded $P$ in $W$ separating its ends and bounding a contractible manifold. But this contradicts $\mu(P) \neq 0$ so is impossible.

Recently Freedman [Fr 2] has extended Casson's methods to obtain the following more definitive result.

THEOREM 6.32'. (1) There is a manifold $W$ which is proper-homotopy equivalent to $S^{3} \times R$ and not diffeomorphic to it.

(2) There is a manifold $P$, proper-homotopy equivalent but not diffeomorphic to $S^{3} \times S^{1}-\{\mathrm{pt}\}$.

(3) There is a proper-homotopy $\mathbf{R}^{4}, Q$, which admits a smooth $\mathbf{Z}_{2}$ action with fixed point set $=($ Poincaré homology sphere $)-\{\mathrm{pt}\}$. Furthermore either $Q$ has a topologically exotic end (and so is not homeomorphic to $\mathbf{R}^{4}$ ) or there is a topological spin 4-manifold of index 8.

(4) Any homology 3-sphere bounds an open 4-manifold $X^{4}$, such that $X^{4}$ is proper homotopy equivalent to a contractible manifold - $\{$ interior $\mathrm{pt}\}$.

Freedman's result is based on an 'inversion' of Casson's Lemma 6.30. That is he shows that if $V$ is as in Theorem 6.29 it is possible to find a compact 'pseudospine' $C$ which is topologically $S^{2} \vee S^{2}$ and is smoothly embedded in 
$V$, except at the wedge point, such that $V-C$ is proper homotopy equivalent to $S^{3} \times R^{1}$. In particular we have the following result:

THEOREM $6.29^{\prime}$. (1) Suppose $W^{4}$ is a simply-connected compact smooth 4-manifold and $\alpha, \beta \in H_{2}(W, Z)$ with $\alpha^{2}=\beta^{2}=0$ and $\alpha \cdot \beta=1$. Let $H$ be the complement in $H_{2}(W, Z)$ of the subspace generated by $\{\alpha, \beta\}$ and let $L$ be the intersection form of $W$ restricted to $H$. Then there exists a simply-connected open submanifold $X \subset W$ with one end, which is proper homotopy equivalent to $S^{3} \times R$ such that the intersection form of $X$ is isomorphic to $L$.

(2) Let $P$ be any simply-connected (compact) Poincaré space of dimension 4 and suppose $P$ has a manifold 0-skeleton. Let $P^{-}$be $P$ minus a point from the interior of this zero cell. Then there exists a smooth manifold proper-homotopy equivalent to $\mathrm{P}^{-}$.

In particular there exist smooth manifolds $E, E^{\prime}$ each having only one end, which is proper homotopy equivalent to $S^{3} \times R$, such that $L_{E} \approx E_{8} \oplus E_{8}$ and $L_{E^{\prime}} \simeq E_{16}$. We shall reduce the proof of Casson's Lemma 6.30 to a series of sublemmas whose proofs, shown to us by Casson, we give in detail. For the proofs of Freedman's theorems, which use the same methods as those we shall use in proving Lemma 6.30 see [Fr 2], [Sb 5].

Lemma 6.33 (CASSOn [Cas 2], [Cas 3]). Suppose $W^{4}$ is a smooth simply-connected 4-manifold and $f: D^{2} \rightarrow W$ is a proper immersion with $f \mid \partial D^{2}$ an embedding such that there exist $\alpha \in H_{2}(W)$ with $[f] \cdot \alpha= \pm 1$. Then $f$ is regular homotopic rel d to a proper immersion $g: D^{2} \rightarrow W$ with $\pi_{1}\left(W-g\left(D^{4}\right)\right)$ $=1$.

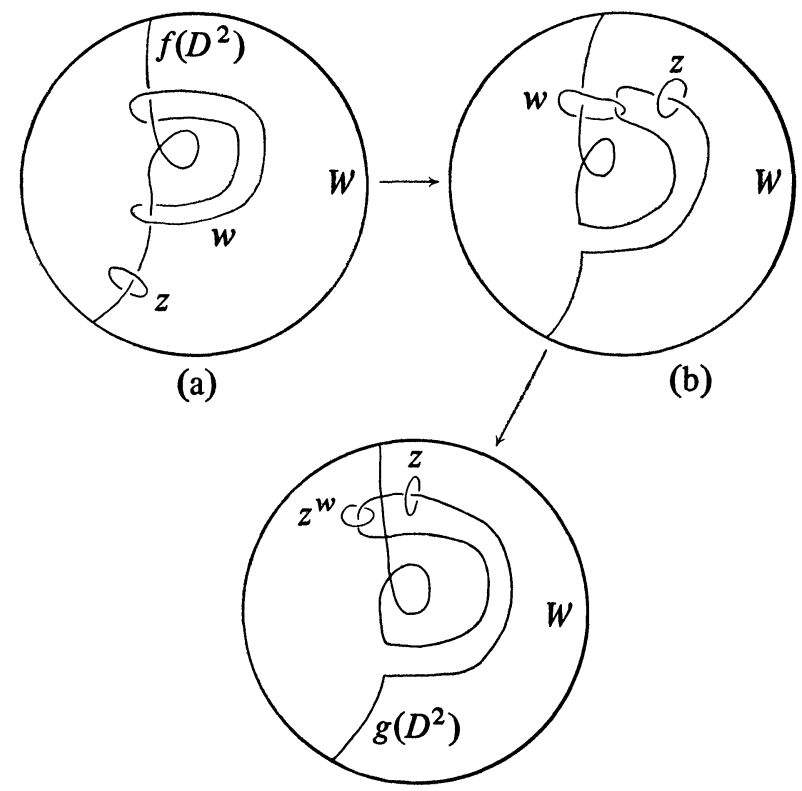

(c)

A proper homotopy of $f\left(D^{2}\right)$ to $g\left(D^{2}\right)$ obtained by pushing along the loop $w$. 
(All immersions are assumed to be $n$-point immersions for some $n$, in this and all succeeding statements.)

Note. Actually the above lemma is true for arbitrary 4-manifolds under the hypothesis that there exists $\alpha \in H_{2}(\tilde{W})$ with $\Sigma_{\mu \in \pi_{1}(W)}[\tilde{f}] \cdot \alpha^{\mu}=1$, where $\tilde{W}$ is the universal cover of $W$ and $\tilde{f}$ is a lift of $f$. In this case the conclusion is $W-g\left(D^{4}\right) \hookrightarrow W$ induces an isomorphism of $\pi_{1}\left(W-g\left(D^{4}\right)\right)$ and $\pi_{1}(W)$.

PROOF OF 6.33. Since $[f] \cdot \alpha= \pm 1$ a straightforward homology calculation shows that $H_{1}\left(W-f\left(D^{4}\right)\right)=0$. Thus $K=\pi_{1}\left(W-f\left(D^{4}\right)\right)$ is perfect. Picking an appropriate basepoint for $\pi_{1}(W-f(D))$, let $Z$ be the element of $K$ represented by a meridian of a tubular neighborhood of $f\left(D^{4}\right)$. Using van Kampen's theorem we see $K$ is normally generated by $Z$. Then using the perfectness of $K$ we obtain that $Z=\left[Z^{u_{1}}, Z^{v_{1}}\right] \ldots\left[Z^{u_{n}}, Z^{v_{n}}\right]$ for some $u_{i}$, $v_{i} \in K$. Thus $K$ is generated by conjugates of $\left[Z, Z^{w_{1}}\right], \ldots,\left[Z, Z^{w_{n}}\right]$, where $w_{i}=v_{i} u_{i}^{-1}$. It thus suffices to show that given any $w \in K$ there will be a regular homotopy (rel $S^{1}$ ) of $f$ to some proper immersion $g: D^{2} \rightarrow W$ such that $Z^{w}$ commutes with $Z$ in $W-g\left(D^{2}\right)$. But if $g$ is constructed by pushing $f$ along $w$ as in Figure 6.4 then it is readily verifiable that $\left[Z, Z^{w}\right]=1$ in a tubular neighbor of $g\left(D^{2}\right)$.

In the obvious fashion we now obtain:

COROLlary $6.33^{\prime}$. Let $W^{4}$ be a smooth simply-connected 4-manifold and the $f_{i}: D^{2} \rightarrow W, i=1, \ldots, n$ proper immersions with $f_{i} \mid \partial D^{2}$ embeddings and $\left[f_{i}\right] \cdot\left[f_{j}\right]=0$ all $i \neq j$ such that there exist $\alpha_{i}, i=1, \ldots, n$, satisfying $\left[f_{i}\right] \cdot \alpha_{j}$ $=\delta_{i j}$. Then the $f_{i}$ are regularly homotopic rel $\partial$ to proper disjoint immersions $g_{i}$ : $D^{2} \rightarrow W$ with $\pi_{1}\left(W-\cup g_{i}\left(D^{2}\right)\right)=\{1\}$.

(Note that, conversely, $\pi_{1}\left(W-g\left(D^{4}\right)\right)=1$ implies there exists $\beta \in$ $H_{2}(W)$ with $[g] \cdot \beta=1$. In fact, this is equivalent to $H_{1}\left(W-g\left(D^{4}\right)\right)=0$.)

Lemma 6.34. Let $W$ be a 4-manifold and let $f: D^{2} \rightarrow W$ be a proper n-point immersion, for some $n$, with $f\left(D^{2}\right)$ having regular neighborhood $N$ in $W$.

Then there are disjoint framed circles $T_{1}, \ldots, T_{n}$ in $\partial N-f\left(S^{1}\right)$ such that if $H$ is obtained from $N$ by attaching 2-handles on $T_{1}, \ldots, T_{n}$ then $\left(H, f\left(S^{1}\right)\right) \simeq$ $\left(B^{4}\right.$, unknotted circle). Further $T_{1}, \ldots, T_{N}$ can be compatible with any given spin structure on $N$.

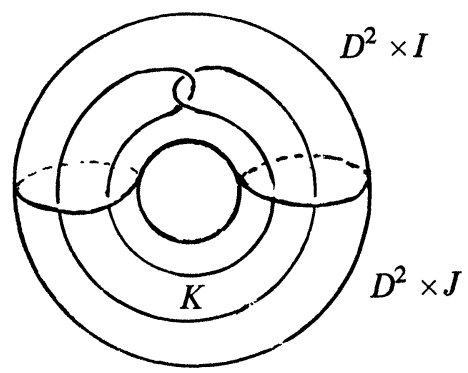

FIGURE 6.5 
Proof. We first note that the diffeomorphism type of $\left(N, f\left(D^{2}\right)\right)$ is completely determined by the number of positive double points $p$, and negative double points $q$, of the immersion $f$. It thus suffices to prove the lemma for the case $n=p=1$ since the more general case can then be taken care of by means of a connected sum and orientation reversal argument.

Thus suppose $f$ has one positive double point. Let $K$ be the knot of $S^{1}$ in $D^{2} \times S^{1}$ shown in Figure 6.5. Identify $I$ with an arc in $S^{1}$ and let $J$ $=\overline{S^{1}-I}$. Let $i: D^{2} \rightarrow \partial D^{3}$ be an embedding, inducing the embedding $i$ : $D^{2} \times S^{1} \rightarrow \partial D^{3} \times S^{1}$.

$K \cap\left(D^{2} \times I\right)$ has two components $K_{0}, K_{1}$, meeting $D^{2} \times 0, D^{2} \times 1$, respectively. Let $A_{0}, A_{1}$ be unknotted arcs in $D^{3} \times \partial I$ such that $\partial A_{t}=i\left(\partial K_{t}\right)$, $(t=0,1)$. Let $B_{0}, B_{1}$ be discs in $D^{3} \times I$ intersecting transversely once, with $\partial B_{t}=A_{t} \cup i\left(K_{t}\right),(t=0,1)$, and such that $D^{3} \times I$ is a regular neighborhood of $B_{0} \cup B_{1}$. Let $B_{2}$ be an unknotted disc in $D^{3} \times J$ such that $\partial B_{2}=A_{0} \cup A_{1}$ $\cup i\left(K \cap\left(D^{2} \times J\right)\right) . B_{2}$ can be chosen so that $B_{0} \cup B_{1} \cup B_{2}$ is the image of a transverse immersion $f_{+}: D^{2} \rightarrow D^{3} \times S^{1}$ with one double point. If the orientation of $D^{3} \times S^{1}$ is compatible with the right-handed orientation in Figure 6.5 the double point of $f_{+}$has positive sign.

Observe that $D^{3} \times S^{1}$ is a regular neighborhood of $f_{+}(D)$. Let $a \in \partial D-$ $i\left(D^{2}\right)$ and let $T=a \times S^{1} \subset \partial\left(D^{3} \times S^{1}\right) ; T$ inherits a framing from the product structure of $\partial\left(D^{3} \times S^{1}\right)$. If $H_{+}$is obtained from $D^{3} \times S^{1}$ by attaching a 2-handle on $T$, then $\left(H_{+}, f_{+}\left(S^{1}\right)\right) \cong\left(B^{4}\right.$, unknot).

But $\left(H_{+}, f_{+}\left(S^{1}\right)\right) \simeq\left(H, f\left(S^{1}\right)\right)$ so our first assertion is verified.

To see that $T$ can be compatible with any given spin structure on $N$, choose a disc neighborhood $B^{4}$ of the double point of $f$ so that $R=\overline{N-B^{4}}$ is a regular neighborhood of $P=\overline{f\left(D^{2}\right)-B^{4}}$. Then $R \sim P \times D^{2}$ and any $D^{2}$ bundle automorphism of $R$ fixing $\partial P \times D^{2}$ extends to an automorphism of $N$ fixing a neighborhood of $f\left(S^{1}\right)$ in $\partial N$. It is easy to choose such an automorphism $h$ for which $h(T)$ is compatible with the given spin structure.

The open sets required for Lemma 6.30 will be constructed as monotone unions of compact submanifolds. These compact submanifolds will be obtained by an inductive process, described in terms of the following definition. Let $S_{1}, \ldots, S_{k}$ be disjoint circles in the boundary of a simply-connected 4-manifold $W$. An envelope for $S_{1}, \ldots, S_{k}$ is a compact submanifold $N$ of $W$ such that

(1) each component of $N$ contains exactly one of $S_{1}, \ldots, S_{k}$,

(2) $N \cap \partial W$ is a regular neighborhood of $\cup_{i \leqslant k} S_{i}$ in $W$,

(3) $S_{1}, \ldots, S_{k}$ are null-homotopic in $N$,

(4) $W-N$ is simply-connected,

(5) there are disjoint framed circles $T_{1}, \ldots, T_{n}$ (called bands) in $\partial N-\partial W$ such that, if $H$ is obtained from $N$ by attaching 2-handles on $T_{1}, \ldots, T_{n}$, then $\left(H, \cup_{i \leqslant k} S_{i}\right) \cong \cup_{i \leqslant k}\left(B^{4}\right.$, unknotted circle),

(6) if $W-N$ has a spin structure, then $T_{1}, \ldots, T_{n}$ are compatible with some spin structure on $W$.

LEMMA 6.35. Let $W$ be a simply-connected 4-manifold and let $f_{1}, \ldots, f_{k}$ : $D^{2} \rightarrow W$ satisfy the hypothesis of Lemma 6.30. There are disjoint transverse immersions $g_{1}, \ldots, g_{k}: D^{2} \rightarrow W$ such that $g_{i}$ is homotopic (rel $S^{1}$ ) to $f_{i}$ and any regular neighborhood of $\cup_{i \leqslant k} g_{i}\left(D^{2}\right)$ is an envelope for $f_{1}\left(S^{1}\right), \ldots, f_{k}\left(S^{1}\right)$. 
Proof. Suppose $f_{1}, \ldots, f_{r-1}$ are homotopic (rel $S^{1}$ ) to disjoint transverse immersions $g_{1}, \ldots, g_{r-1}$ with $X_{r}=W-\cup_{i<r} g_{i}\left(D^{2}\right)$ simply-connected. The induction starts with $r=1$ and $X_{1}=W$. Since $g_{i} f_{r}=f_{i} f_{r}=0$ if $i<r, f_{r}$ represents zero in $H_{2}\left(W, X_{r}\right) \cong \pi_{2}\left(W, X_{r}\right)$, so $f_{r}$ is homotopic (rel $\left.S^{1}\right)$ to a map $h_{r}: D^{2} \rightarrow X_{r}$. By hypothesis, there is an element $\alpha_{r} \in H_{2}(W)$ with $\left[f_{r}\right] \alpha_{r}=1,\left[g_{i}\right] \alpha_{r}=\left[f_{i}\right] \alpha_{r}=0$ if $i<r$. It follows that $\alpha_{r}$ is the image of an element $\gamma_{r} \in H_{2}\left(X_{r}\right)$ with $\left[h_{r}\right] \gamma_{r}=1$. By general position and Lemma $6.33 h_{r}$ is homotopic (rel $S^{1}$ ) to a transverse immersion $g_{r}: D^{2} \rightarrow X_{r}$ with $X_{r+1}=X_{r}$ $-g_{r}\left(D^{2}\right)$ simply-connected. By induction, $f_{1}, \ldots, f_{k}$ are homotopic (rel $S^{1}$ ) to disjoint transverse immersions $g_{1}, \ldots, g_{k}$ with $W-\cup_{i<k} g_{i}\left(D^{2}\right)$ simplyconnected.

Let $N$ be a regular neighborhood of $\cup_{i<k} g_{i}\left(D^{2}\right)$ in $W$. By construction and Lemma 6.34, $N$ satisfies condition (1)-(5) in the definition of envelope. To verify condition (6), suppose that $W-N$ has a spin structure. Let $\beta \in H_{2}(W)$, and put $\gamma=\beta-\Sigma_{i \leqslant k}\left(\left[g_{i}\right]\right) \alpha_{i}$. Then $\left[g_{i}\right] \gamma=0 \quad(i=$ $1,2, \ldots, k)$, so $\gamma$ is in the image of $H_{2}(W-N)$. Since $W-N$ has a spin structure, $\gamma^{2}$ is even. By hypothesis, $\alpha_{i}^{2}$ is even, so $\beta^{2}$ is even for all $\beta \in H_{2}(W)$. This implies that $W$ has a spin structure; by Lemma 6.34 $T_{1}, \ldots, T_{n}$ can be compatible with the induced structure on $N$.

LEMMA 6.36. Let $W$ be a simply-connected 4-manifold, let $S_{1}, \ldots, S_{k}$ be disjoint circles in $\partial W$ and let $N$ be an envelope for $S_{1}, \ldots, S_{k}$ with bands $T_{1}, \ldots, T_{n}$. There are disjoint transverse immersions $g_{1}, \ldots, g_{n}: D^{2} \rightarrow \overline{W-N}$ with $g_{i}\left(S^{1}\right)=T_{i}$ such that any regular neighborhood of $N \cup \cup{ }_{i<n} g_{i}\left(D^{2}\right)$ is an envelope for $S_{1}, \ldots, S_{k}$.

The proof requires the following definition. Let $f: D^{2} \rightarrow W$ be a map such that $f \mid S^{1}$ is an embedding into $\partial W$. For any framing $\phi$ of $f\left(S^{1}\right)$ in $\partial W$, let $W_{\phi}$ be the manifold obtained from $W$ by attaching a 2-handle on $\left(f\left(S^{1}\right), \phi\right)$. Let $\alpha_{\phi} \in H_{2}\left(W_{\phi}\right)$ be represented by $f$ together with the core of the handle. There is a unique choice of $\phi$ (up to homotopy) making $\alpha_{\phi}^{2}=0$; this is called the induced framing $f$. Two framings $\phi, \psi$ have a well-defined difference $\phi-\psi \in$ $\pi_{1}\left(\mathrm{SO}_{2}\right) \cong \mathrm{Z}$. Let $s: S^{2} \rightarrow W$ be a map, and let $f \# s: D^{2} \rightarrow W$ be a connected sum of $f$ and $s$. Then $(f \# s)^{*}-f^{*}=2[f][s]+[s]^{2}$.

Proof of Lemma 6.36. Let $X=\overline{W-N}$; since this is simply-connected, there are maps $f_{1}, \ldots, f_{n}: D^{2} \rightarrow X$ with $f_{i}\left(S^{1}\right)=T_{i}$. The first step is to find elements $\alpha_{1}, \ldots, \alpha_{n} \in H_{2}(X)$ with $\left[f_{i}\right] \alpha_{j}=\delta_{i j}$ and $\alpha_{i} \alpha_{j}=0$.

Let $H$ be the manifold obtained from $N$ by attaching 2-handles on $T_{1}, \ldots, T_{n}$; then $H$ is a disjoint union of balls. Let $T_{i}^{\prime} \subset \partial H$ be the belt of the 2-handle attached on $T_{i}$. Then $\partial N-\partial W-\cup_{j \leqslant n} T_{j} \cong \partial H-\cup_{i<k} S_{i}-$ $\cup_{j<n} T_{j}^{\prime}$. Since $S_{1}, \ldots, S_{k}, T_{1}^{\prime}, \ldots, T_{n}^{\prime}$ bound disjoint singular dises in $H$, the linking number of $T_{j}^{\prime}$ with $S_{i}$ or $T_{i}^{\prime}$ is zero (if defined). It follows that the parallels of $T_{1}^{\prime}, \ldots, T_{n}^{\prime}$ are null-homologous in $\partial H-\cup_{i \leqslant k} S_{i}-\bigcup_{j \leqslant n} T_{j}^{\prime}$. Equivalently, the meridians of $T_{1}, \ldots, T_{n}$ are null-homologous in $\partial N-\partial W$ $-\bigcup_{j \leqslant n} T_{j}$.

This implies the existence of elements $\alpha_{1}^{\prime}, \ldots, \alpha_{n}^{\prime} \in H_{2}(\partial N-\partial W)$ with $\left[T_{i}\right] \alpha_{j}^{\prime}=\delta_{i j}$. Let $\alpha_{j}$ be the image of $\alpha_{j}^{\prime}$ in $H_{2}(X)$; then $\left[f_{i}\right] \alpha_{j}=\delta_{i j}$ and $\alpha_{i} \alpha_{j}=0$. The next step is to adjust $f_{1}, \ldots, f_{n}$ so that $f_{i}^{*}$ coincides with the preferred framing on $T_{i}$, and $f_{i} f_{j}=0$ whenever $i \neq j$. 
By condition (6) in the definition of envelope, if $X$ has a spin structure then the preferred framing $\phi_{i}$ on $T_{i}$ is compatible with some spin structure on $W$. But $f_{i}^{*}$ is compatible with any spin structure on $W$, so $\phi_{i}-f_{i}^{*}$ is even. If $X$ has no spin structure, then there is an element $\beta \in H_{2}(X)$ with $\beta^{2}$ odd. Let $s$ : $S^{2} \rightarrow X$ represent $\beta$; then $\left(f_{i} \# s\right)^{*}-f_{i}^{*}$ is odd. Replace $f_{i}$ by $f_{i} \# s$ if necessary to make $\phi_{i}-f_{i}^{*}$ even. Let $\phi_{i}-f_{i}^{*}=2 m_{i}$, and let $s_{i}: S^{2} \rightarrow X$ represent $\alpha_{i}$. Replace $f_{i}$ by $f_{i} \# m_{i} s_{i}$; since $\left[f_{i}\right] \alpha_{i}=1$ and $\alpha_{i}^{2}=0$, this replaces $f^{*}$ by $f_{i}^{*}+2 m_{i}=\phi_{i}$.

Finally replace $f_{i}$ by $f_{i} \# \Sigma_{j<i}\left(-f_{i} f_{j}\right) s_{j}$. Since $\left[f_{i}\right] \alpha_{j}=\delta_{i j}$ and $\alpha_{i} \alpha_{j}=0$, this makes $f_{i} f_{j}$ zero for $i \neq j$ without altering $f_{i}^{*}$. Observe that $f_{1}, \ldots, f_{n}: D^{2} \rightarrow X$ now satisfy all the hypotheses of Lemma 6.30 .

Let $g_{1}, \ldots, g_{n}: D^{2} \rightarrow X$ be the immersions obtained by applying Lemma 6.35 to $f_{1}, \ldots, f_{n}: D^{2} \rightarrow X$. Let $P$ be a regular neighborhood of $\cup_{i<n} g_{i}\left(D^{2}\right)$ in $X$, so small that $P \cap \partial X \subset \partial N$. Since $g_{i}$ is homotopic (rel $S^{1}$ ) to $f_{i}, g_{i}^{*}=f_{i}^{*}$ is the preferred framing on $T_{i}$.

$N \cup P$ satisfies conditions (1)-(4) for an envelope of $S_{1}, \ldots, S_{k}$. By Lemma 6.35, $P$ is an envelope for $T_{1}, \ldots, T_{n}$ in $X$, and by hypothesis $N$ is an envelope for $S_{1}, \ldots, S_{k}$ in $W$. If $X-P$ has a spin structure, then $X$ and $W$ have spin structures. By Lemma 6.34, bands $U_{1}, \ldots, U_{p}$ can be chosen for $P$ compatible with a spin structure on $W$. If $X-P$ has no spin structure, let $U_{1}, \ldots, U_{p}$ be any bands for $P$.

Let $K$ be obtained from $P$ by attaching 2-handles along $U_{1}, \ldots, U_{p}$; then $\left(K, \cup_{i<n} T_{i}\right) \cong \cup_{i<n}\left(B^{4}\right.$, unknot). Since the framing induced on a circle in $S^{3}$ by any null-homotopy in $B^{4}$ is untwisted, the preferred framing $g_{i}^{*}$ on $T_{i}$ corresponds to the untwisted framing of the unknot.

Let $H$ be obtained from $N \cup P$ by attaching 2-handles on $U_{1}, \ldots, U_{p}$, so $H=N \cup K$. The above description of $\left(K, \cup_{i<n} T_{i}\right)$ shows that $H$ is obtained from $N$ by attaching 2-handles on $T_{1}, \ldots, T_{n}$. Since $N$ is an envelope for $S_{1}, \ldots, S_{k},\left(H, \cup_{i<k} S_{i}\right) \cong \cup_{i<k}\left(B^{4}\right.$, unknot). This verifies conditions (5) and (6), so $N \cup P$ is an envelope for $S_{1}, \ldots, S_{k}$ with bands $U_{1}, \ldots, U_{p}$. The result of Lemma 6.36 follows immediately.

Proof of Lemma 6.30. Suppose $f_{1}, \ldots, f_{k}: D^{2} \rightarrow W$ satisfy the hypotheses of Lemma 6.30. Apply Lemma 6.35 to obtain immersions $g_{1}, \ldots, g_{k}: D^{2} \rightarrow$ $W$, and let $N_{1}$ be a regular neighborhood of $U_{i<k} g_{i}\left(D^{2}\right)$. Suppose an envelope $N_{r}$ for $f_{1}\left(S^{1}\right), \ldots, f_{k}\left(S^{1}\right)$ has been constructed with bands $T_{1}, \ldots, T_{n}$. Apply Lemma 6.36 to obtain disjoint transverse immersions $h_{1}, \ldots, h_{n}: D^{2} \rightarrow W-N_{r}$ with $h_{i}\left(S^{1}\right)=T_{i}$, and let $N_{r+1}$ be a regular neighborhood of $N_{r} \cup \cup{ }_{i<n} h_{i}\left(D^{2}\right)$. Since $N_{r+1}$ is an envelope for $f_{1}\left(S^{1}\right), \ldots, f_{k}\left(S^{1}\right)$, this process yields an infinite sequence $N_{1} \subset N_{2} \subset \cdots$ of envelopes.

Let $V=\cup_{r=1} N_{r}$ and let $V_{i}$ be the component of $V$ containing $f_{i}\left(S^{1}\right)$. Since $N_{r}$ is contained in the interior of $N_{r+1}, V$ is an open set. Clearly $V_{i} \cap \partial W$ is an open regular neighborhood of $f_{i}\left(S^{1}\right)$ and $f_{i}$ is homotopic (rel $\left.S^{1}\right)$ to a map into $V_{i}$. It remains to prove that $\left(V_{i}, V_{i} \cap \partial W\right)$ has the proper homotopy type of $\left(D^{2} \times \mathbf{R}^{2}, S^{1} \times \mathbf{R}^{2}\right)$. Assume for simplicity that $k=1$, so $V$ is connected; in general the components of $V$ can be treated separately. Of course, the envelope $N_{r}$ may still have several bands $T_{1}, \ldots, T_{n}$. 
By condition (5) in the definition of envelope, $\pi_{1}\left(N_{r}\right)$ is generated by conjugates of $\left[T_{1}\right], \ldots,\left[T_{n}\right]$. Since $T_{i}$ is null-homotopic in $N_{r+1}$, inclusion induces the zero map $\pi_{1}\left(N_{r}\right) \rightarrow \pi_{1}\left(N_{r+1}\right)$. By condition (5) again, $H_{i}\left(N_{r}\right)=0$ for $i \geqslant 2$, it follows that $V$ is contractible.

Let $Y_{r, s}=\bar{N}_{s}-N_{r}$ and $M_{r}=\overline{\partial N_{r}-\partial W}$, so $Y_{r, s}$ is a regular neighborhood of the union of $M_{r}$ and a 2-complex. It follows that inclusion induces a surjection $\pi_{1}\left(M_{s}\right) \rightarrow \pi_{1}\left(Y_{r, s}\right)$.

The most important consequence of condition (5) is that the manifold obtained from $M_{r}$ by surgery on $T_{1}, \ldots, T_{n}$ is diffeomorphic to $S^{1} \times D^{2}$. This implies that the quotient of $\pi_{1}\left(M_{r}\right)$ by the subgroups generated by conjugates of $\left[T_{1}\right], \ldots,\left[T_{n}\right]$ is cyclic. The quotient is generated by a meridian $z_{r}$ to $g_{1}\left(S^{1}\right)$ in $\partial M_{r}$, and is infinite since it maps onto $H_{1}\left(H-g\left(D^{2}\right)\right)$. It follows that the map $\pi_{1}\left(M_{r}\right) \rightarrow \pi_{1}\left(Y_{r, s}\right)$ induced by inclusion has infinite cyclic image generated by $z_{r}$.

If $r<s<t$ then $Y_{r, t}=Y_{r, s} \cup Y_{s, t}$; by van Kampen's theorem, the map $\pi_{1}\left(Y_{r, s}\right) \rightarrow \pi_{1}\left(Y_{r, t}\right)$ induced by inclusion has infinite cyclic image generated by $z_{r}$. Let $Y_{r}=\bigcup_{s=r}^{\infty} Y_{r, s}$; then $\pi_{1}\left(Y_{r}\right)$ is infinite cyclic, generated by $Z_{r}$. Observe that $Y_{1} \supset Y_{2} \supset \ldots$ is a base of neighborhoods for the end of $V$. Since $z_{r}$ is homotopic to $z_{r-1}$ in $Y_{r-1}$, inclusion induces an isomorphism $\pi_{1}\left(Y_{r}\right) \rightarrow$ $\pi_{1}\left(Y_{r-1}\right)$. It follows that the end of $V$ has an infinite cyclic fundamental group.

We close this chapter with an alternate construction of the manifold $W$ in Theorem 6.32 using the link calculus which is more geometrically appealing [Kirb 6]. We first construct a manifold $Q$ as follows: Let $X \subset S^{1} \times$ int $B^{2}$ be the Whitehead continuum (see [RIf, p. 182], [W 1]), defined as $X=$ $\cap_{i>0} \alpha^{i}\left(S^{1} \times B^{2}\right)$, where $\alpha: S^{1} \times B^{2} \rightarrow S^{1} \times B^{2}$ is the imbedding shown below ( $\alpha$ should have 0 -twisting about its core)

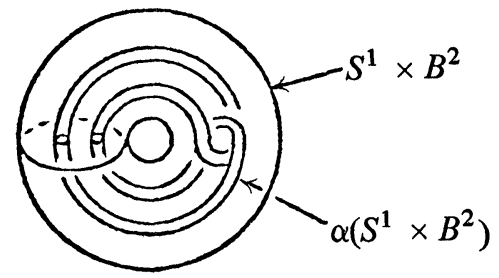

Regarding $S^{2} \times S^{2}$ as the union of a 0-handle $B_{0}^{4}$, two 2-handles $B_{1}^{2} \times D^{2}$, $B_{2}^{2} \times D^{2}$ and a 4-handle $B_{3}^{4}$, define a compact subset $C=B_{3}^{4} \cup c X_{1} \cup c X_{2}$, where each $X_{i}$ is a Whitehead continuum constructed in int $B_{i}^{2} \times \partial D^{2} \subset \partial B_{3}^{4}$, the cocore attaching tube of the $i$ th 2-handle, and where $X_{i}$ is coned off to the center of the 2-handle $B_{i}^{2} \times D^{2}$. Then $Q=S^{2} \times S^{2}-C$.

(The open subset $B^{2} \times$ int $D^{2}-c X_{i}$ of the open 2-handle $B^{2} \times$ int $D^{2}$ is then a flexible 2-handle. In fact it is the simplest possible flexible 2-handle. The manifold $Q$ can then be regarded as the union of an open 0-handle int $B_{0}^{4}$ and two flexible 2-handles.) Now either $Q$ is not diffeomorphic to some $S^{2} \times S^{2}-\{\mathrm{pt}\}$ and we are finished or one can again show that there exists a smooth open manifold $W$ with the proper homotopy type of $S^{3} \times R$ but not diffeomorphic to it. The question of whether or not $Q$ is diffeomorphic or not to $S^{2} \times S^{2}-\{\mathrm{pt}\}$ can be reduced to a question in knot theory as follows. 
Let $L_{0}$ be the Whitehead link as pictured below

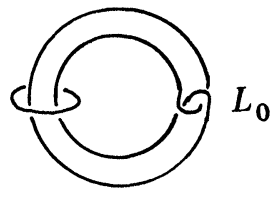

and let $L_{1}$ be its untwisted double

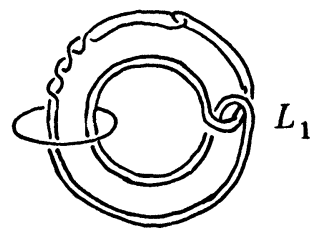

This construction can be iterated by replacing $O$ by $O$ or call the $n$th iterate $W_{n}$.

Then $Q$ is diffeomorphic to $S^{2} \times S^{2}-\{\mathrm{pt}\}$ if and only if for some $n \geqslant 0$, $W_{n}$ is null-concordant. (Recall that a link $L$ in $S^{3}$ is null-concordant if and only if it bounds a disjoint union of 2-discs in $D^{4}$ with $\partial D^{4}=S^{3} \supset L$.)

Note that the compact subset $C=S^{2} \times S^{2}-Q$ is cell-like (i.e., homotopic to a point in any arbitrarily small neighborhood) and satisfies the cellularity criterion (i.e., $Q$ is 1 -connected at $\infty$ ); (see [McM]). However, $C$ is smoothly cellular ( $\equiv$ the intersection of a nested sequence of smooth 4-balls) $\Leftrightarrow$ some $W_{n}$ is null-concordant.

\section{CHAPTER 7. FOUR MANIFOLDS AS SUB AND SUPER-MANIFOLDS}

7.1 Representing 2-dimensional homology classes of 4-manifolds. As we pointed out in the preceding chapter, it was shown by Kervaire, Milnor and Rohlin [KM 1], [R 2] that there exist 1-connected closed smooth 4-manifolds $M$ with $\xi \in H_{2}(M)$ not representable by a smoothly embedded sphere. Although not relevant to the problem of doing surgery in dimension 4 the question of representing such $\xi$ is still of interest.

More particularly we have the following questions.

(I) What is the minimum genus of a smoothly embedded connected orientable surface $F_{g}$ which can represent $\xi$.

(II) How does the allowable minimum genus change if we require that $F_{g}$ be only PL or just topologically (nonlocally flatly) embedded. (Note that by Wall [Wa 6] every locally flat PL embedding $F^{2} \hookrightarrow M^{4}$ is isotopic to a smooth embedding $F^{2} \hookrightarrow M^{4}$.)

In this section we consider the first question and discuss question II in \$7.2. In discussing question 1 we first note that, as a consequence of [Thm] every $\xi$ can be represented by some smoothly embedded orientable surface. (The analogous result is not necessarily true in higher-dimensions. See [Thm].) 
There have been two essentially different approaches to the question of realizing $\xi$. One approach ([Tr], [MM 3], [At 1]) uses the connection between 4-manifolds and algebraic invariants of links and the other (Hsiang and Szczarba, Rohlin) [HSZ, R 2] is a more direct use of cobordism theory and the Atiyah-Singer $G$-signature theorem.

We begin with the second approach to the problem of realizability. In [R 2] Rohlin proves the following result:

THEOREM 7.1. Let $X$ be a smooth connected closed 4-manifold with $H_{1}(X)=$ 0 . Let $F$ be an oriented connected closed two dimensional submanifold of $X$ realizing $\xi \in \mathrm{H}_{2}(X)$ with genus $(F)=g$ and set $b_{2}(X)=\mathrm{rk} H_{2}(X ; Z)$.

Then

(1) if $\xi$ is divisible by 2 then

$$
\left|\xi^{2} / 2-\sigma(X)\right|-b_{2}(X)<2 g
$$

(2) if $p$ is an odd prime and $\xi$ is divisible by $k=p^{n}$, then

$$
\left|\left(\left(k^{2}-1\right) / 2 k^{2}\right) \xi^{2}-\sigma(x)\right|-b_{2}(X) \leqslant 2 g .
$$

(Hsiang and Szczarba proved a somewhat weaker version of the above in [HSZ] by essentially similar methods. We discuss only Rohlin's result.)

As a corollary one can obtain for example:

CoRollary 7.2. (1) Let $X=\mathbf{C} P^{2}$ with $\gamma$ the generator of $H_{2}\left(\mathbf{C} P^{2}\right)$. Suppose $\xi=n \gamma$. Then

(i) if $2 \mid n ; g \geqslant \frac{1}{4} n^{2}-1$ while

(ii) if $h \mid n, h$ an odd prime power, then $g \geqslant\left(\frac{1}{4} n^{2}-1\right)-\left(n^{2} / 4 h^{2}\right)$.

(2) Let $X=S^{2} \times S^{2}$ with $\xi_{1}$, $\xi_{2}$ natural generators of $H_{2}\left(S^{2} \times S^{2}\right)$. Suppose $\xi=n_{1} \xi_{1}+n_{2} \xi_{2}$

(i) if $2 \mid n_{1}$ and $2\left|n_{2} ; g \geqslant \frac{1}{2}\right| n_{1} n_{2} \mid-1$

(ii) if $h\left|n_{1}, h\right| n_{2}, \quad h$ a prime power and $h$ odd then $g>\frac{1}{2}\left|n_{1} n_{2}\right|-1-\frac{1}{2}\left|n_{1} n_{2}\right| / h^{2}$.

Note that in case (1) $\xi$ can be realized by algebraic curves of genus $\frac{1}{2}(n-1)(n-2)$ if $n \neq 0$ and there is no known realization by a surface of lower genus (Thom conjectures that there is no lower realization).

Similarly in case (2) $\xi$ can be realized by algebraic curves of genus $\left(\left|n_{1}\right|-1\right)\left(\left|n_{2}\right|-1\right)$ and no lower genus realization is known. Both in cases (1) and (2) the upper bound on the realization is about twice as large as the lower bound obtained above. One should also note that Theorem 7.1 says nothing about the possibility of representing $\xi$ by a nonlocally flat sphere and one obtains no information about PL realizability of 2-homology classes. Theorem 7.1 is also not a generalization of Theorem 1 of [KM 1] (see our Lemma 1.13) giving more information in some cases and less in others. (In particular Corollary 7.2 gives far more information about realizability for $S^{2} \times S^{2}$ or $\mathbf{C} P^{2}$ than does Corollary 1.13. However for $X=\mathbf{C} P^{2} \# \mathbf{C} P^{2}$ with $\gamma_{1}, \gamma_{2}$ the obvious generators of $H_{2}(X)$, Corollary 1.13 implies that $\gamma_{1}+3 \gamma_{2}$ is not realizable by an embedded sphere while Theorem 7.1 says nothing about this case.) Note that by 6.5 both $\gamma_{1}$ and $3 \gamma_{2}$ are realizable by smoothly embedded spheres. 
The proof of Theorem 7.1 goes as follows.

(a) Let $F$ be a surface realizing $\xi \in H_{2}(X)$. For any $m$ dividing $\xi$ it is possible to construct an $m$-fold cyclic branched covering manifold $Y$ of $X$ branched over $F$. Let $\pi: Y \rightarrow X$ denote the canonical projection.

(b) Let $U=X-T(F)$ and $V=\left.Y\right|_{U}$ the corresponding unbranched $m$ sheeted cover. $(T(F)$ a tubular neighborhood of $F$ in $X$.)

One then computes using only simple homology theory that

$$
\begin{aligned}
& b_{2}(Y)=m b_{2}(X)+2(m-1) g+2 b_{1}(V), \\
& b_{1}(Y)=b_{1}(V) .
\end{aligned}
$$

One can show by algebraic means that if $m=2$ or $m$ is an odd prime power then $b_{1}(V)=0$.

(c) $Y$ is an $m$-fold cyclic branched covering space and thus there is a natural action of $\mathrm{Z}_{m}$ on $Y$ with $Y / \mathrm{Z}_{m}=X$. Let $H=H_{2}(Y ; C)$ and notice that the intersection pairing $\cdot$ on $H_{2}(Y ; Z)$ extends naturally to a Hermitian pairing on $H$. Let $\mathcal{T}: Y \rightarrow Y$ be the natural generator of the group $\mathrm{Z}_{m}$ of covering transformations of $Y(\mathcal{T}$ is the covering transformation which rotates the fibers of the normal disc bundle $\tilde{T}$ of $\tilde{F}=\pi^{-1}(F)$ through an angle of $2 \pi / m)$ and let $\sigma_{*}: H \rightarrow H$ be the induced isometry of $(H, \cdot)$ on itself. Note that $\mathfrak{T}_{*}^{m}=$ id and writing $\omega=e^{2 \pi i / m}$ let $E_{r}$ be the $\omega^{r}$-eigenspace of $\mathcal{T}, 0<r<m$. Then $(H, \cdot)$ decomposes as an orthogonal direct sum

$$
H=E_{0} \oplus \cdots \oplus E_{m-1}
$$

and letting $\sigma_{r}(Y)$ be the signature of $\cdot$ restricted to $E_{r}$ one can obtain (see Rohlin [R 2], Casson and Gordon [CG]).

$$
\sigma_{r}(Y)=\sigma(X)-\frac{2 \xi^{2} r(m-r)}{m^{2}} .
$$

(The proof of (**) relies heavily on the 4-dimensional $G$-signature theorem.)

(d) Again on strictly algebraic grounds one can obtain that

$$
\operatorname{dim}\left(E_{r}\right)=\left\{\begin{array}{l}
b_{2}(X) \text { if } r=0, \\
b_{2}(X)+2 g \text { if } r>0 .
\end{array}\right.
$$

Thus clearly for all $r, 0 \leqslant r<m$.

$$
\left|\sigma(x)-\frac{2 \xi^{2} r(m-r)}{m^{2}}\right| \leqslant b_{2}(X)+2 g
$$

and the desired inequalities follow by taking $r=1$ if $m=2$ and $r=k$ if $m=2 k+1$.

Although rather far away from the problem of finding embedded spheres to represent homology classes it is still of interest to see what kind of estimates one can get on the genus of a nonorientable 2-manifold $F$ representing a homology class. (Recall that if $F$ is nonorientable then $g(F)=2-$ $\chi(F), \chi(F)$ the Euler characteristic of $F$.) In this case Rohlin shows by methods analogous to those he used for the orientable case that: 
THEOREM 7.3. Suppose $X$ is as in Theorem 7.1 and $A$ is a nonorientable surface realizing the zero element of $H_{2}(X ; \mathrm{Z})$.

Then

$$
\left|\frac{1}{2} a-\sigma(X)\right|-b_{2}(X) \leqslant g
$$

where $a$ is the Euler number of the normal bundle of $A$ (the so called 'normal Euler number' of $A$ ).

Corollary 7.3' (SeE [Whit 2], [Mas]). Suppose $F$ is a closed nonorientable 2-manifold and $i: F \hookrightarrow S^{4}$ a smooth embedding.

Then $|a| \leqslant 2 g$, where $a$ is the normal Euler number of $F$. Furthermore, for every integer $n$ with $|n| \leqslant 2 g$ and $n \equiv 2 g(\bmod 4)$ there exists an embedding $i_{n}$ : $F \hookrightarrow S^{4}$ with the normal Euler number of $i_{n}(F)$ equal to $n$.

Proof. The inequality $|a| \leqslant 2 g$ follows from Theorem 7.1' applied to $X=S^{4}$. The realizability follows by noting that (i) If $F=\mathbf{R} P^{2}$ then for any embedding $F \hookrightarrow S^{4}$ we have $a(F)= \pm 2$ depending only on the orientation of $S^{4}$. (ii) Every nonorientable surface $F$ equals $\#_{i=1}^{q}\left(\mathbf{R} P^{2}\right)_{i}$ and by taking connected sums of $\left(S^{4}, \mathbf{R} P^{2}\right)$ and $\left(S^{4},-\mathbf{R} P^{2}\right)$ one can achieve all possible $a_{F}$ in $-2 g,-2 g+4, \ldots, 2 g-4,2 g$ as desired.

One can also relate the Euler number of $a$ to the signature of $M$ and thus prove a nonorientable version of Theorem 1.12.

In particular if $M^{4}$ is an oriented, connected closed 4-manifold with $H_{1}\left(M^{4} ; \mathbf{Z}\right)=0$ and $F^{2}$ is a closed not-necessarily orientable surface realizing a characteristic element in $H_{2}(M ; Z)$ then one can define a quadratic function [Mt 1] $\phi: H_{1}\left(F^{2} ; \mathbf{Z}_{2}\right) \rightarrow \mathbf{Z}_{4}$. [In this context quadratic means $\phi(u+v)=$ $\phi(u)+\phi(v)+2 \mu(u, v), \mu$ the intersection pairing on $H_{1}\left(F^{2} ; \mathbf{Z}_{2}\right)=H$.] Then following E. H. Brown [Brwn], one can define an Arf type invariant $K(\phi) \in$ $\mathbf{Z}_{8}$ associated to $\phi$ by

$$
\sum_{\mu \in H} i^{\phi(u)}=\sqrt{2}^{\operatorname{dim} H}\left(\frac{1+i}{\sqrt{2}}\right)^{K(\phi)} .
$$

One can then prove

(1) $K\left(F^{2}\right)$ is an isotopy invariant of $F^{2}$.

(2) $K\left(F^{2}\right) \equiv \chi\left(F^{2}\right)(\bmod 2)$, where $\chi\left(F^{2}\right)$ is the Euler characteristic of $F^{2}$.

(3) If $F^{2}$ is orientable, $K\left(F^{2}\right)=4 \operatorname{Arf}\left(F^{2}\right)$, where $4: \mathbf{Z}_{2} \rightarrow \mathbf{Z}_{8}$ is the nontrivial homomorphism.

One then has the following theorem of Guillou and Marin [GM]

Theorem 7.4. [NONORIENTABle Rohlin] (COMPare With TheORem 1.12). Let $a\left(F^{2}\right)$ be the normal Euler number of the number $F^{2} \hookrightarrow M^{4}$ then

$$
\sigma\left(M^{4}\right) \equiv a\left(F^{2}\right)+2 K\left(F^{2}\right)(\bmod 16) .
$$

Corollary 7.5. (The Generalized Whitney CONGRUENCE [R 3]).

$$
\sigma\left(M^{4}\right) \equiv a\left(F^{2}\right)+2 \chi\left(F^{2}\right)(\bmod 4)
$$

We now recall the connection between the problem of realizing $\xi$ and numerical link invariants. Thus suppose $X$ is a smooth oriented compact 4-manifold with $H_{1}(X)=0$. Let $\xi \in H_{2}(X)$ and suppose $\bar{F}$ is a connected 
oriented 2-manifold realizing $\xi$. Let $T$ be a regular neighborhood of the 2-skeleton $K$ of $X$ in some cell-decomposition of $X$. Then by general position we may assume that $\bar{F}$ intersects $K$ and $T$ transversely and lies within some slightly larger regular neighborhood $T^{\prime}$ of $K$ such that $\overline{T^{\prime}-T} \approx \partial T \times I$. Then by the assumed transversality of $\bar{F}$ and $T$ we have that $\bar{F} \cap T$ consists of some number of disjoint smoothly embedded discs in $T$ with $\bar{F} \cap \partial T$ their boundary circles. Set $L=\bar{F} \cap \partial T, M=\partial T$ and $F=\bar{F}-\bar{F} \cap T$. Then $F$ is simply $\bar{F}$ with a number of discs removed and so $F$ and $\bar{F}$ have the same genus $g$. Now $L$ is a smooth link in the 3-manifold $M$ and bounds an oriented compact 2-manifold $F \subset M \times I$. Thus the problem of establishing a minimum bound for the genus of $\bar{F}$ is equivalent to determining the minimum genus of an orientable surface $F \subset M \times I$ bounding a given link $L \subset M$. In many cases of interest $X$ has a handle decomposition with no 3-handles. $M$ is then simply $S^{3}$ and $L$ is thus a classical link $L \subset S^{3}$. The problem of whether $\xi$ is representable by a sphere is then exactly equivalent to whether $L$ is a weak slice link in the sense of ([Fox, p. 173-Property 4], [Tr, p. 163]). (By [M 3] $X$ will have such a decomposition whenever it is, for example a complete intersection surface. In particular $\mathbf{C} P^{2}, S^{2} \times S^{2}$ and $V_{4}$ have such handle decompositions. See our discussion in Chapter 3.)

In the classical case (where $M=S^{3}$ ) Tristram obtained some estimates on the minimum genus of a surface $F$ in $S_{+}^{4}=$ (upper hemisphere of $S^{4}$ ) bounding a link $L$ in $S^{3}=\partial S_{+}^{4}$.
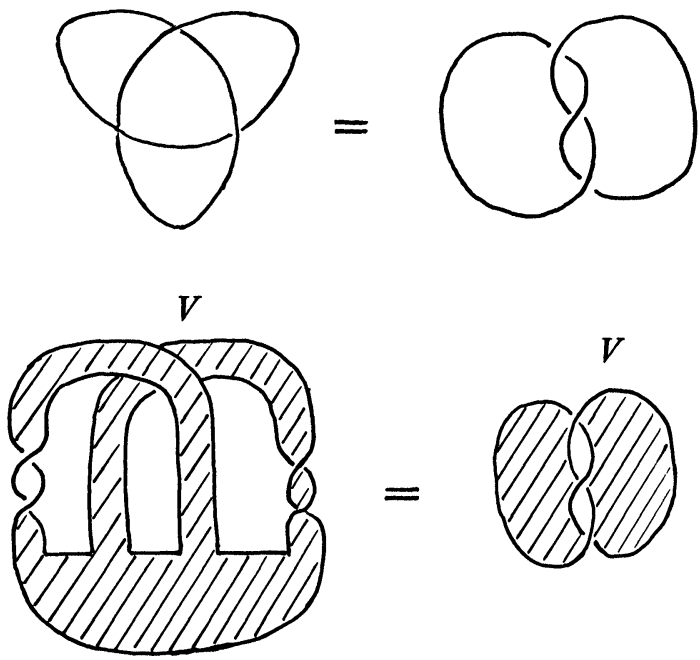

$$
\begin{aligned}
& M_{V} \\
& \| \begin{array}{rr}
-1 & 1 \\
0 & -1
\end{array} \mid
\end{aligned}
$$

FIGURE 7.1 
We recall that if $L$ is a link in $S^{3}$ there will always exist an orientable embedded 2-manifold $V$ in $S^{3}$ with $\partial V=L . V$ is called a Seifert surface for $L$. Furthermore the interior of $V, \dot{V}$ can always be bicollared in $S^{3}$ to obtain an embedding of $\dot{V} \times[-1,1]$ in $S^{3}-L$. Then if $x \in H_{1}(\dot{V})$ is represented by a 1-cycle $x$ in $\dot{V}$ we let $x^{+}$denote the homology class carried by $x \times 1$ in $\dot{V} \times[-1,1]$.

We then define the Seifert form $f_{V}$ of $L$ by

$$
f_{V}(x, y)=l k\left(x, y^{+}\right) \text {for } x, y \in H_{1}(\dot{v}) .
$$

Corresponding to any choice of basis for $H_{1}(\dot{V})$ we also have the Seifert matrix $M_{V}$ of $L$. (Example: If $L$ is the right-handed trefoil knot, then in Figure 7.1 we exhibit a Seifert surface $V$ and associated Seifert matrix $M_{V}$.) (see [Fox]). Note that $M_{V}$ induces a bilinear form on $\mathbf{Z}^{2 g} \times \mathbf{Z}^{2 \boldsymbol{g}} \rightarrow \mathbf{Z}$, where $g$

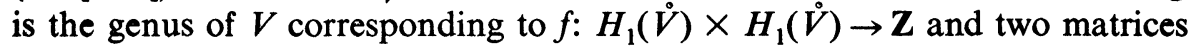
$M_{V}, M_{V^{\prime}}$ correspond to the same form if and only if they are congruent.

Now suppose $\left(l, \mathbf{Z}^{n}, \mathbf{Z}\right)$ is any bilinear form and $\omega$ a complex number with $|\omega|=1$ and $\omega \neq 1$. Let ${ }^{\omega} l$ be the form on $Z^{n} \times Z^{n}$ to $C$ defined by

$$
{ }^{\omega} l(x, y)=(1-\bar{\omega})\{l(x, y)-\omega l(y, x)\} .
$$

(If $M$ is a matrix representative of $l$ then $M+M^{t}-\omega M^{t}-\bar{\omega} M$ will be a matrix representative of ${ }^{\omega} l$.) It is easy to verify that ${ }^{\omega} l$ will always be a Hermitian form and thus have a well-defined signature which we denote by $\sigma_{\omega}(l)$.

Now for any link $L \subset S^{3}$ and Seifert surface $V$ for $L$ we can form ${ }^{\omega} f_{V}$ as above. We let $\sigma_{\omega}(V)$ denote the signature and $n_{\omega}(V)$ denote $1+$ nullity $\left({ }^{\omega} f_{V}\right)$ (nullity ${ }^{\omega} f_{V}=2 g-$ rk $M_{V}$ ). If $\omega$ is a $p^{k}$ th root ( $p$ prime, $k=1$ if $p=2$ ) of unity $\neq 1$ one can show that $\sigma_{\omega}(V), n_{\omega}(V)$ are in fact independent of the choice of Seifert surface $V$ for $L$ and thus for any primitive $p$ th root of unity $\xi$ one has numerical link invariants which we denote by $\sigma_{\omega}(L), n_{\omega}(L)$. (If $\omega=-1$ we write $\sigma_{2}(L), n_{2}(L)$ instead of $\sigma_{-1}(L), n_{-1}(L)$ and if $p=2 m+1$, $\omega=\xi^{m}, \xi=e^{2 \pi i / p}$ then $\sigma_{\omega}(L), n_{\omega}(L)$ are sometimes written as $\sigma_{p}(L), n_{p}(L)$.)

In [Tr] Tristram shows that if $F$ is a smooth connected surface spanning the link $L$ in $S_{+}^{4}$ then

$$
\left|\sigma_{p}(L)\right|+n_{p}(L)-\mu(L) \leqslant 2 g_{F}
$$

where $\mu(L)=$ number of components of $L$ and $g_{F}$ is the genus of $F$. (Actually Tristram's proof shows that for any prime $p$, and any $p^{k}$ th root of unity $\omega \neq 1$ $(k=1$ if $p=2)$

$$
\left.\left|\sigma_{\omega}(L)\right|+n_{\omega}(L)-\mu(L) \leqslant 2 g_{F} .\right)
$$

Thus by reducing the problem of representing $\xi \in H_{2}(X)$ to a problem in link theory one can get estimates on the minimum genus of $\bar{F}$ representing $\xi$. In particular as direct corollaries of (6) one finds the following generalization of Examples 1 and 2 in [KM 1]. 
Corollary 7.2'. (1) Let $a, b$ denote the generators of $H_{2}\left(S^{2} \times S^{2}\right)$ represented by $S^{2} \times *$ and $* \times S^{2}$, respectively. Suppose $\xi=q a+r b \in H_{2}\left(S^{2} \times\right.$ $\left.S^{2}\right)$ with g.c.d. $(q, r)>1$.

Then $\xi$ is not representable by an embedded sphere.

(2) Let $\gamma$ be the generator of $\mathrm{H}_{2}\left(\mathrm{CP}^{2}\right)$.

Suppose $\xi=n \gamma$. Then if $n \geqslant 3, \xi$ is not representable by an embedded sphere.

(Actually, as we shall see shortly, Tristram's inequality contains significantly more information than is contained in this theorem. In fact it also implies Corollary 7.2 of Theorem 7.1.)

Now suppose $M$ is not a 3-sphere but an arbitrary 3-manifold and $L$ is a link homologous to zero in $H_{1}(M)$. Then as in [MM 3] one can show that $L$ has an orientable spanning surface $V$ in $M$. However as opposed to the case when $M=S^{3}$ not all such spanning surfaces will be homologous in $H_{2}(M, L)$ but will differ by elements of $H_{2}(M)$. In [MM 3] it is shown how one can still define a Seifert form $f_{V}$ associated to the Seifert surface $V$ of $L$ and then define $\sigma_{\omega}(V), n_{\omega}(V)$ as the case of classical links. Now however, although $\sigma_{\omega}(V), n_{\omega}(V)$ will be independent of the particular choice of $V$ in a given homology class $\mathcal{T}$ in $H_{2}(M, L)$ it might depend on the choice of homology class $\mathcal{T}$. Thus one can define numerical link invariants devoted by $\sigma_{\omega}(L, \mathcal{T})$, $n_{\omega}(L, \mathscr{T})$.

Analogous to Tristram's inequality one obtains the following result:

THEOREM 7.6 [MM 3]. Let $M$ be a compact oriented 3-manifold with $H_{1}(M)$ free and suppose $L$ is a link in $M$ homologous to zero. Let $F$ be a spanning surface for $L$ in $M \times I$ and let $\mathcal{T}$ denote the image of the homology class of $F$ in $H_{2}(M, L)$. Then if $\omega$ is a $p^{k}$ th root of unity $\neq 1, p$ a prime and $k=1$ if $p=2$ we have

$$
\left|\sigma_{\omega}(L, \mathcal{T})\right|+n_{\omega}(L, \mathcal{T})+b_{1}(M)-\mu(L)-\delta(L)<2 g_{F}
$$

where $b_{1}(M)=$ rk $H_{1}(M, Z)$ and $\delta(L)=$ rank of a minimal generating set of $H_{1}(M, \mathbf{Z}) / i_{*} H_{1}(L, Z)$.

In particular if $H_{1}(L) \stackrel{i}{\rightarrow} H_{1}(M)$ is onto

$$
\sigma_{\omega}(L, \mathscr{T})+n_{\omega}(L, \mathcal{T})+b_{1}(M)-\mu(L)<2 g_{F} .
$$

(Notice that if $M$ is a homotopy 3-sphere ( $\left.7^{\prime}\right)$ becomes (6') again.)

As a consequence of Theorem 7.5 one has in principle a technique for estimating the minimum genus of $\xi \in H_{2}(X)$ for a 4-manifold $X$ with $H_{1}(\partial T)$ free. ( $X$ need not satisfy $H_{1}(X)=0$. Recall $T$ is a regular neighborhood of some 2-skeleton of $X$.) How do the estimates in $\left(6^{\prime}\right)$ and $\left(7^{\prime}\right)$ compare to those of (1) and (2) when $H_{1}(X)=0$ ?

In [MM 1] it is shown that if $M=S^{3}$ then

$$
\sigma_{2}(L)=\sigma(X)-\frac{1}{2} \xi^{2} \text { and } n_{2}(L)=\mu(L)-b_{2}(X) .
$$

Thus (6') implies (1) and both approaches to the problem of estimating the minimal genus come up with the same number in the case $M=S^{3}$.

As an interesting aside on the type of information not provided by either Rohlin's theorem or the results of [Tr], [MM 3] we note the following fact pointed out by Akbulut [At 2]. 
Suppose $K$ is a framed knot in $S^{3}$ with framing $r \in \mathbf{Z}$ and let $K^{r}$ be the 4-manifold it represents. Then using Tristram's theorem (equation (6)) one can show (see [At 1]) that if the $p$-signature $\sigma_{p}(K)$ of $K$ is nonzero for a prime number dividing $r$ then $\mathrm{H}_{2}\left(K^{r}\right)$ cannot be represented by a smoothly embedded sphere. However let $K$ be the knot in Figure 3.10 of Chapter 3. Then it can be shown by direct calculation that $\sigma_{p}(K) \neq 0$ all prime $p$ but the generator of $\mathrm{H}_{2}\left(\mathrm{~K}^{+1}\right)$ is representable by a smoothly embedded sphere! In fact Akbulut shows that the manifolds $K^{+1}$ and $R^{+1}$, where $R$ is also pictured in Figure 3.10 of Chapter 3 are the same and that $R$ is a slice knot. Thus the generator of $\mathrm{H}_{2}\left(R^{+1}\right)=$ the generator of $\mathrm{H}_{2}\left(K^{+}\right)$is representable by a smooth sphere even though $\sigma_{p}(K) \neq 0$. Thus, for example if $M^{4}$ is a 4-manifold and $F^{2}$ is a 2-homology class with $F^{2} \cdot F^{2}= \pm 1$ which is representable by a PL embedded sphere then the knot cobordism invariants of the link of the singularity of $F^{2}$ in $M^{4}$ can't be obstructions to representing $F^{2}$ by a smoothly embedded sphere. We note however that by [CS 10] if $\sigma_{p}(K) \neq 0$ for any $p$, then the generator of $\mathrm{H}_{2}\left(K^{0}\right)$ is not representable by a smoothly embedded sphere. Thus if $F^{2}$ above satisfies $F^{2} \cdot F^{2}=0$ the knot cobordism invariants are almost exactly the obstructions to representing $F^{2}$ by a smoothly embedded sphere. See [CS 5], [CS 8], [CS 10], [CS 13] for details.

7.2 PL-representations. We now consider question II. That is, how much better can we do if we allow PL (nonlocally flat) embeddings to represent our homology classes? The first result in that direction is also in [KM 1]. We have:

THEOREM 7.7. In $S^{2} \times S^{2}$ or $\mathbf{C P}^{2}$ every 2-dimensional homology class can be represented by a PL-embedded sphere.

We give an algebraic proof for $\mathbf{C} P^{2}$. A similar proof can be given for $S^{2} \times S^{2}$. Thus let $\gamma$ be the canonical generator of $\mathbf{C} P^{2}$ and suppose $\xi=n \gamma$ for some $n \geqslant 1$. (If $n$ is negative we construct a spherical representative for $-n \gamma$ and then simply change orientation.) Then if $[x: y: z]$ are projective coordinates for $\mathbf{C} P^{2}$ it is easily verified that the curve $C: X^{n}+Y^{n-1} Z=0$ is a PL-embedded sphere representing $n \gamma$. (Note that $X^{n}+Y^{n-1} Z$ can be thought of in the following way: Decompose $C P^{2}$ as the +1 disc bundle $U$ over $S^{2}$ and a 4-disc $V$ (i.e., $D^{4}$ with a 2-handle attached via $\bigcirc_{+1}$ and an additional 4-handle). Then one can check that $K=C \cap \partial U=C \cap \partial V$ is an $(n, n-1)$-torus knot in $S^{3}, C \cap U=D_{U}$ is a smooth 2-disc in $U$ with $\partial D_{U}=K$ and $C \cap V$ is the cone on $K$ in $V=D^{4}$. Thus $C$ has one bad point, namely the vertex of the cone $C \cap V$.)

(We point out that although the above theorem shows that all classes in $\mathrm{H}_{2}\left(S^{2} \times S^{2}: \mathrm{Z}\right)$ can be represented by PL-spheres we have no information at all on the minimum genus of a smooth representative of $2 a+3 b$ in $\mathrm{H}_{2}\left(\mathrm{~S}^{2} \times\right.$ $\left.S^{2} ; \mathbf{Z}\right)$ ! If we translate this question into the corresponding link-theoretic question we obtain a link $L_{k, l}$ such as in Figure 7.2A. Note that if $l=0$ or $k \leqslant 1$ then the Tristram inequality (6) rules out the possibility that $L_{k, l}$ bounds a punctured 2-sphere in $B^{4}$. The next simplest case is $k=2, l=1$, pictured in Figure 7.2B. Even for this simple case the minimum genus of the punctured surface it bounds in $B^{4}$ is unknown!) 


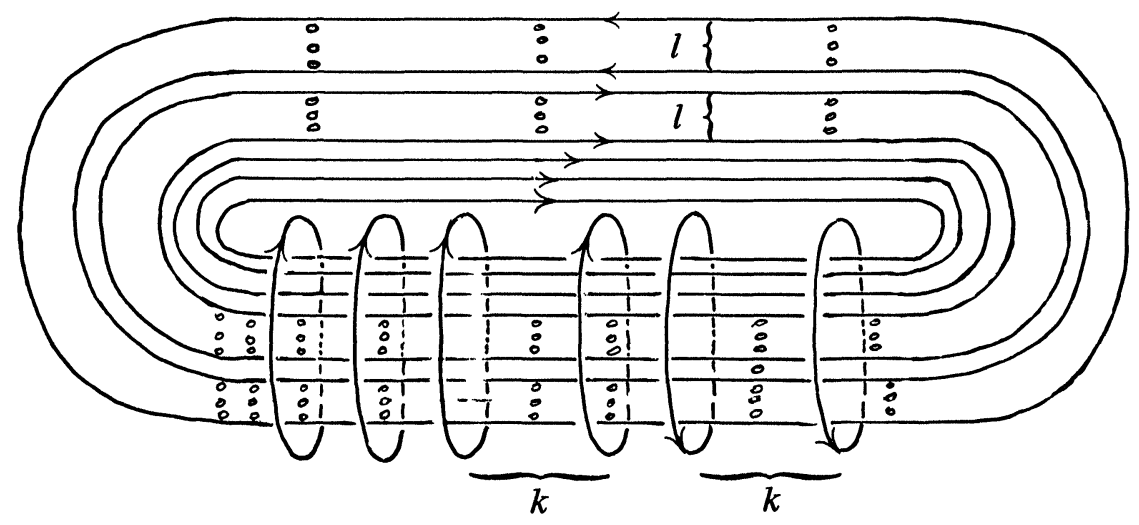

FIGURE 7.2A

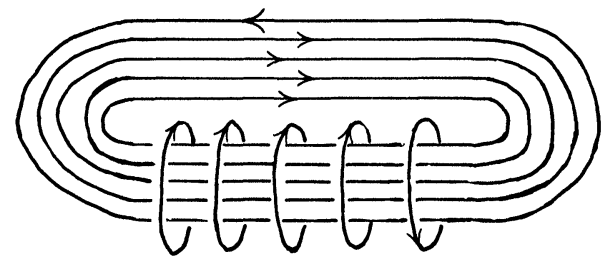

FIGURE 7.2B

Returning to question of PL-embeddings we have the following more general theorem about PL-representations.

Proposition 7.8. Let $X$ be a closed PL 4-manifold containing a 2-dimensional subcomplex $K$ such that

(1) $K$ is acyclic.

(2) The boundary of a regular neighborhood $T$ of $K$ is $S^{3}$. Then every homology class $\xi \in \mathrm{H}_{2}(M ; Z)$ can be represented by a PL-embedded sphere.

Note that if $X$ has a handlebody decomposition with no 3-handles then it clearly satisfies the hypothesis of the above proposition. Clearly then if $X$ has a special handlebody decomposition (in the sense of Chapter 3) it satisfies the requisite hypothesis. (It is as yet unknown whether every 1-connected 4-manifold has a handlebody decomposition with no 3-handles.) To prove Proposition 7.8 we recall by our discussion earlier in this section that if $F$ is any smooth manifold representing $\xi$ we can by a general position argument insure that $F \cap T$ is a finite number of discs $D_{1}, \ldots, D_{\mu}$ and $F \cap \partial T$ a link in $S^{3}$ with disjoint components $K_{1}, \ldots, K_{\mu}$. By connecting the components by disjoint bands we can without altering the homology class of $F$ connect up the various $D_{i}$ to produce a 2-cycle $F^{*}$ homologous to $F$ with $F^{*} \cap T$ a 2-disc and $\partial\left(F^{*} \cap T\right)=F^{*} \cap \partial T$ a knot $K$ in $S^{3}$. Coning off $K$ in a regular neighborhood $N$ of $\partial T$ in $M-T$ provides us with the requisite PL 2-sphere homologous to $F^{*}$ and thus representing $\xi$. 
The analogue of Proposition 7.8 for 4-manifolds with boundary $\neq S^{3}$ is not true! In fact Akbulut [At 2] proves:

Proposition 7.9. There exists a compact 4-manifold $W^{4}$ (with boundary) which is homotopy equivalent to $W_{0}^{4} \# \mathbf{C} P^{2}\left(W_{0}^{4}\right.$ a $Q$-homology ball) such that the generator $\xi \in H_{2}(W ; Z) \approx Z$ cannot be represented even by a PL-embedded sphere.

We will not give the proof of this theorem but simply note that it is based on use of the Casson-Gordon invariant of [CG]. Full details can be found in [At 2]. In Figure 7.3 we give link pictures of the manifolds $W_{0}, W$, and indicate which homology classes are not representable.

Similar in spirit to the above is an example of Matsumoto and Venema, [Mt V], of an open subset $W^{\prime}$ of $\mathbf{R}^{4}$ which has the homotopy type of $S^{2}$ but such that no nontrivial class in $H_{2}\left(W^{\prime} ; Z\right)$ can be represented by a PL-embedded 2-sphere. (A standard basis of $H_{2}\left(W^{\prime} \# S^{2} \times S^{1} ; Z\right)$ can, however, even be represented by smoothly embedded 2 -spheres.)

We note that the same failure of the Whitney trick which does not allow us to represent 2-dimensional homology classes of a 1-connected 4-manifold $X$ by embedded spheres can also prevent one from geometrically separating two smooth spheres in $X$ which are algebraically separated.

More precisely there exists a compact closed 1-connected 4-manifold $X$ and $\xi_{1}, \xi_{2} \in H_{2}(X)$ such that $\xi_{1} \cdot \xi_{2}=0, \xi_{1}, \xi_{2}$ are representable by smoothly embedded 2-spheres but if $S_{1}, S_{2}$ are any such 2-spheres then $S_{1} \cap S_{2} \neq \varnothing$.
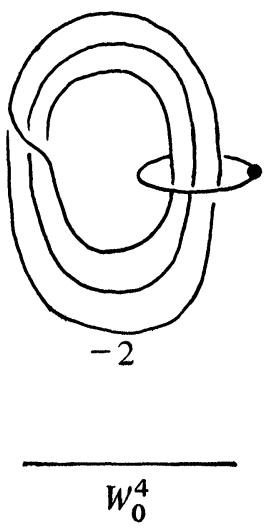



$w=w_{0}^{4} \# \mathbf{C} P^{2}$

Note: $H_{*}\left(W_{0}^{4} ; \mathbf{Q}\right)=H_{*}\left(D^{4} ; \mathbf{Q}\right)$. The homology class $[\xi]$, corresponding to the unknot $\xi$ above generates $H_{2}(W ; Z)$ and is not representable by a PL-embedded sphere. 
In fact we have the following example of [KM 1].

Let $\gamma_{1}, \gamma_{2}$ be the canonical generators of $H_{2}\left(\mathbf{C} P^{2} \# \mathbf{C} P^{2} ; \mathbf{Z}\right)$. Then by [Bord], $3 \gamma_{2}$ can be represented by a smoothly embedded sphere while clearly $\gamma_{1}$ can be so represented. However although $\gamma_{1} \cdot 3 \gamma_{2}=0$ it is never possible to separate smooth spheres representing them! (Suppose such a separation were possible. Then if $S_{1}^{*}$, represents $\gamma_{1}$ and $S_{2}$ represents $3 \gamma_{2}$ we can pipe $S^{*}$ and $S_{2}$ together to get a smoothly embedded 2 -sphere $S$ representing $\gamma_{1}+3 \gamma_{2}$. But $\gamma_{1}+3 \gamma_{2}$ is characteristic for $\mathbf{C} P^{2} \# \mathbf{C} P^{2}$ and thus by the KervaireMilnor theorem cannot be so represented.)

Similar results can be obtained even if we reduce our demands to having the relevant homology classes represented by only PL (nonlocally flat)-embedded spheres. The same methods used to prove Proposition 7.9 also give:

Proposition 7.10 (AKBULUT) [At 2]. There exists a compact spin 4-manifold $M^{4}$ (with boundary) which is homotopy equivalent to $M_{0}^{4} \# S^{2} \times S^{2}$ where $M_{0}^{4}$ is a homology $S^{1} \times D^{3}$ such that:

(i) $\tilde{M}^{4}$ is $\mathrm{Z}[\mathrm{Z}]$-homology equivalent to $\left(M_{0}^{4} \# S^{2} \times S^{2}\right)$ (where $\sim$ denotes infinite cyclic cover) and

(ii) there are hyperbolic classes $\xi_{1}, \xi_{2} \in H_{2}(M, Z[Z])$ that cannot be represented by a PL-embedded $S^{2} \vee S^{2}$. In fact there is no embedded homology $S^{2} \times S^{2}-$ Int $B^{4}$ in the interior of $M^{4}$ representing $\left(\xi_{1}, \xi_{2}\right)$.

The manifolds $M_{0}, M$ are exhibited (via framed link pictures) in Figure 7.4.

In particular any choice of PL-embedded spheres $S_{1}, S_{2}$ representing $\xi_{1}, \xi_{2}$ must then have card $\left(S_{1} \cap S_{2}\right)>1$. (A similar proposition has been proven by Freedman in [Fr 1] using somewhat different methods.)
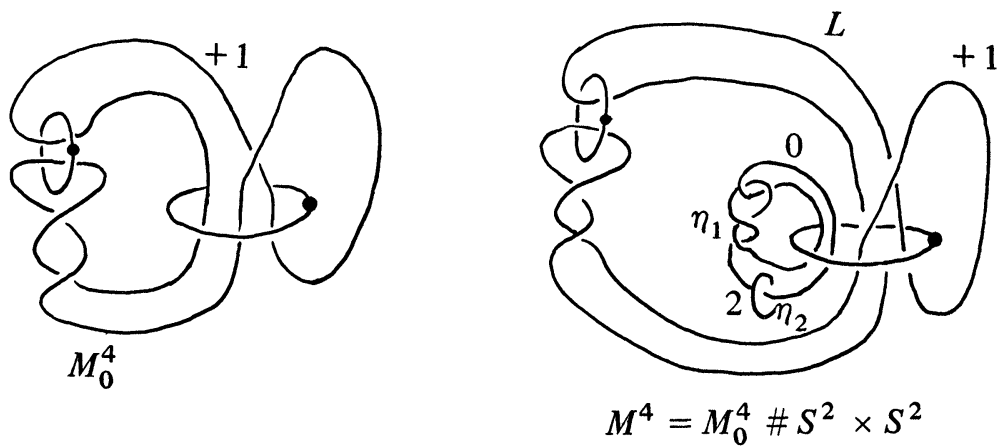

Let $\xi_{1}, \xi_{2}$ be the homology classes corresponding to the components $\eta_{1}, \eta_{2}-\eta_{1}$ of the link $L$ above. It is easy to see that $\left\{\xi_{1}, \xi_{2}\right\}$ are a hyperbolic pair and it can be shown that they are not even PL-representable by $S^{2} \times S^{2}$. Clearly both $\eta_{1}, \eta_{2}$ are individually representable by $S^{2}$ 's.

\section{FIGURE 7.4}

Proposition 7.10 is in some sense more devastating to any hope of mimicing higher-dimensional surgery techniques in dimension 4 than the nonembedding results cited earlier, as it shows that 'surgery kernels' cannot always be PL, let alone smoothly, represented by geometrically hyperbolic sphere pairs, even though the individual homology classes can be represented. 
Similar examples of algebraically but not geometrically disjoint homology classes can also be constructed. We recall that in Chapter 1 we defined the Arf invariant of any characteristic element of $\mathrm{H}_{2}(W), W$ a 1-connected compact smooth 4-manifold. Then Matsumoto [Mt 4] has shown by straightforward geometric calculations of the appropriate Arf invariants that:

Proposition 7.11 (Matsumoto, [Mt 4]). Suppose $W$ is a simply-connected compact smooth 4-manifold with

(i) $X_{1}, \ldots, X_{n} \in H_{2}(W ; Z)$ characteristic,

(ii) $X_{i}+X_{j}$ characteristic for all $i<j$,

(iii) $\Sigma X_{i}$ characteristic.

(1) If the $X_{i}$ are represented by disjointly embedded PL 2-spheres, then $\operatorname{Arf}\left(\Sigma X_{i}\right) \equiv \Sigma \operatorname{Arf}\left(X_{i}\right)(\bmod 2)$.

(2) If the $X_{i}$ are represented by continuous maps with pairwise disjoint images, then

$$
\operatorname{Arf}\left(\sum_{i=1}^{n} X_{i}\right) \equiv \sum_{i<j} \operatorname{Arf}\left(X_{i}+X_{j}\right)+n\left(\sum_{i=1}^{n} \operatorname{Arf}\left(X_{i}\right)\right)
$$

EXAMPLE. (1) In Figure 7.5 let $\left[X_{1}\right],\left[X_{2}\right]$ be the 2-homology classes corresponding to the components $X_{1}, X_{2}$ of the framed link $L$ representing a 4-manifold $W$. It is easy to see that $\left[X_{1}\right],\left[X_{2}\right],\left[X_{1}\right]+\left[X_{2}\right]$ are characteristic. Now $l k\left(X_{1}, X_{2}\right)=0$ so $\left[X_{1}\right] \cdot\left[X_{2}\right]=0$. But $\operatorname{Arf}\left(\left[X_{1}\right]\right)=\operatorname{Arf}\left(\left[X_{2}\right]\right)=0$ and $\operatorname{Arf}\left(\left[X_{1}\right]+\left[X_{2}\right]\right)=1$ so $\left[X_{1}\right],\left[X_{2}\right]$ cannot be represented by disjointly embedded PL-spheres.

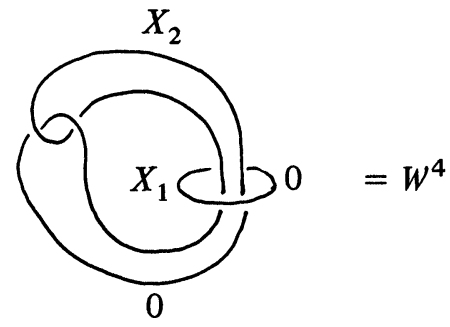

FiguRE 7.5

(2) Let $L_{n}$ be the framed link of $n$ components pictured in Figure 7.6 and suppose $M_{n}$ is the associated 4-manifold. Let $\xi_{i}=\left[X_{i}\right]$ be the elements of $H_{2}\left(M_{n}\right)$ corresponding to the components $X_{i}$ of $L$. that

Then $\xi_{i}, \xi_{i}+\xi_{j}, \Sigma \xi_{i}$ are all characteristic and an explicit calculation shows

$$
\begin{gathered}
\operatorname{Arf}\left(\xi_{1}+\cdots+\xi_{n}\right)= \begin{cases}1, & n=3, \\
0, & n \geqslant 4 .\end{cases} \\
\operatorname{Arf}\left(\xi_{i}\right)=\operatorname{Arf}\left(\xi_{i}+\xi_{j}\right)=0 \quad(i<j) .
\end{gathered}
$$

Thus if $n=3, \xi_{1}, \xi_{2}, \xi_{3}$ cannot be represented by continuous maps with pairwise disjoint images, even though $\xi_{i} \cdot \xi_{j}=l k\left(X_{i}, X_{j}\right)=0$ for all $i, j$. (If $n \geqslant 4$ it is not clear what the situation will be. In [Kob] K. Kobayashi proves a homotopic version of the Whitney trick and uses it to show that two homology classes $\xi_{1}, \xi_{2} \in H_{2}(M), M$ 1-connected, with $\xi_{1} \cdot \xi_{2}=0$ can always 
be homotopically separated. Thus $n=3$ is the minimum number for the above phenomena.)

We give one last example [Mt 4] illustrating the mess arising in 4-manifold theory due to the failure of the Whitney trick.
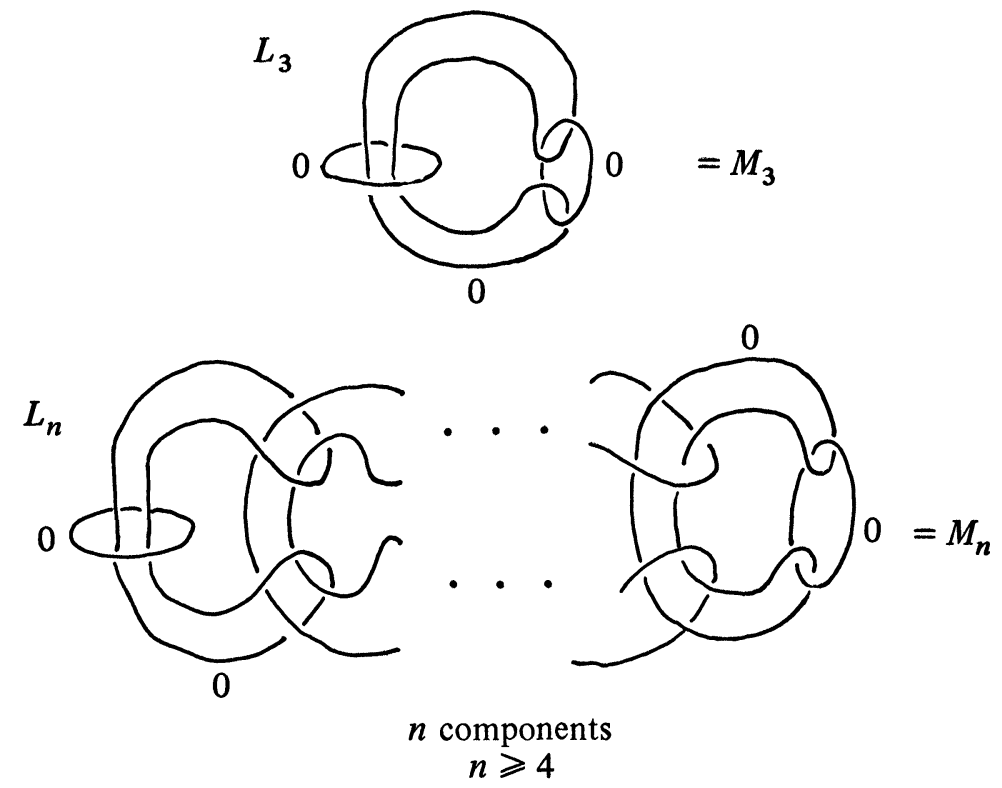

FIGURE 7.6

Example (Matsumoto, [Mt 4]). We recall that the Kummer surface $V_{4}$ can be constructed by taking $T^{4} / \sigma$, where $\sigma$ is the canonical involution $\sigma\left(Z_{1}, Z_{2}, Z_{3}, Z_{4}\right)=\left(\bar{Z}_{1}, \bar{Z}_{2}, \bar{Z}_{3}, \bar{Z}_{4}\right)$ and resolving the 16 singular points $p_{i}$, as described in Chapter 2. ( $V_{4}$ is $W_{1}$ in the notation used there.) In the process of resolving the singularities of $T^{4} / \sigma$ we introduce 16 smoothly embedded 2-spheres $L_{i}$ (i.e. the zero-sections of the -2 disc bundles used to replace neighborhoods of the $\left.p_{i}\right)$ such that $L_{i} \cdot L_{j}=-2 \delta_{i j}$. Now $b_{2}\left(V_{4}\right)=22$ and there clearly exist homology classes $0 \neq \xi \in H_{2}\left(V_{4}\right)$ with $\xi \cdot L_{i}=0$ all $i$ [simply lift a class in $T^{4} / \sigma$ which does not contain the $p_{i}$ ]. Now $V_{4}$ is simply connected so $\xi$ can always be represented by a continuous map $f: S^{2} \rightarrow V_{4}$.

Claim. There exists no homotopy of $f$ to a map $g$ such that $g\left(S^{2}\right) \cap$ $\left(\cup_{i=1}^{16} L_{i}\right)=\varnothing$.

Proof. Suppose such a map $g$ existed. Now $V_{4}-\cup L_{i}=T^{4} / \sigma-$ $\left\{p_{1}, \ldots, p_{16}\right\}$ so $g$ would lift to a map $\tilde{g}: S^{2} \rightarrow T^{4}-\{16$ points $\}$. But $\pi_{2}\left(T^{4}\right.$ - points $)=0$ so $\tilde{g}$ must be null homotopic contradicting $\xi \neq 0$.

7.3 Surgery and embedding phenomena. One of the major contributions of surgery theory is to provide new tools for answering when one can embed a manifold $M^{m}$ into another manifold $V^{m+q}$. To translate this problem into terms amenable for surgery calculations suppose that $f: M^{m} \rightarrow V^{m+q}$ is an embedding. For simplicity let us temporarily assume that $f$ is smooth. Let $D$ be a closed tubular neighborhood of $M$ in $V$ and let $E$ be the closure of its 
complement. Note that $D$ is essentially the disc bundle $D(\nu)$ of the normal bundle $\nu$ of $M$ in $V$, and we clearly then have a diffeomorphism of $E \cup D(\nu)$ and $V$.

If we wish to translate these data into homotopy theoretic terms we could say that any embedding $f: M^{m} \rightarrow V^{m+q}$ determines

(1) a $(q-1)$-spherical fibration over $M(S(\nu)$ in our case),

(2) a finite CW complex $E \supset S(\nu)$,

(3) a simple homotopy equivalence $h: E \cup D(\nu) \rightarrow V$.

Note that $D(\nu)$ is of course diffeomorphic to the mapping cylinder $M(p)$ of $p: S(\nu) \rightarrow M$.

This motivates the following definition.

Definition (PE) Suppose $M^{m}, V^{m+q}$ are compact manifolds. Then a Poincare embedding $\theta$ of $M$ in $V$ is

(1) a $(q-1)$-spherical fibration $\xi=(S, M, P)$ with projection $p: S \rightarrow M$,

(2) a (finite) CW-complex $E \supset S$,

(3) a simple homotopy equivalence $h: E \cup M(p) \rightarrow V$

where $M(p)$ is the mapping cylinder of $p$, and $E \cap M(p)=S$. We will let $\xi_{\theta}$, $E_{\theta}, h_{\theta}$ denote the $\xi, E, h$ associated to a Poincaré embedding $\theta$.

(If $q=2$ then since $G(2) / O(2)$ is contractible we can replace (1) by the existence of a 2-plane bundle $\xi$ over $M$. The parts of $S$ and $M(p)$ are then of course played by the sphere bundle $S(\xi)$ and disc bundle $D(\xi)$ associated to $\xi$.) If $\xi$ is an oriented bundle we will say the Poincare embedding is oriented. Although the motivation for our definition above shows only that smooth embeddings determine Poincare embedding one can show that PL or TOP embeddings do also. (See [Wa 3, §11] for the PL or TOP locally-flat case and [CS 5] for general PL embeddings if $q=2$.)

We note that the concept of a Poincare embedding is a homotopy theoretic concept and could as well have been defined on the level of Poincaré complexes. (We could define a relative version for embeddings $(M, \partial M) \rightarrow$ $(V, \partial V)$ in the obvious fashion, but suppress such considerations for simplicity. We point out however that all the theorems we shall quote have relative analogues. See [Wa 3, §11], for details if $q \geqslant 3$ and [Cap 3], [CS 5] if $q<2$.)

The fundamental questions we now discuss are

(1) Suppose there exists a Poincaré embedding $\theta$ of $M^{m}$ into $V^{m+q}$. When is $\theta$ induced by a smooth or PL embedding $f: M^{m} \hookrightarrow V^{m+q}$.

(2) Suppose $f: M^{m} \rightarrow V^{m+q}$ is a homotopy equivalence. When is $f$ homotopic to an embedding?

To answer 1 and 2 above we consider the related splitting problem for simple homotopy equivalences.

Thus suppose that $f: M^{n} \rightarrow X^{n}$ is a simple homotopy equivalence between closed manifolds and $Y^{m} \subset X$ is a connected locally-flat submanifold.

We say that $f$ is splittable (actually $s$ or simply-splittable) along $Y$ if $f$ is homotopic to a map $g$ transverse to $Y$ such that $g \mid g^{-1} Y: g^{-1} Y \rightarrow Y$ is a simple homotopy equivalence. We say $g$ is (simply) split (along $Y$ ).

(In the above definition we have assumed $\partial X=\partial M=\partial Y=\varnothing$. We could have admitted boundaries at the cost of adding some complications to the definition. Henceforth we assume all our manifolds are unbounded. We could also have stated the definition and subsequent theorems under the weaker 
assumption that $X, Y$ were a Poincaré complex with $Y$ having a linear normal bundle in $X$.)

Now suppose $f: M^{n} \rightarrow X^{n}$ and $Y^{m}$ are as above. We can by general position always assume that $f$ is already transverse regular to $Y$. Then as in [Wa 3, §11] there is a natural bundle map $b$ with domain the stable normal bundle of $f^{-1} Y$, covering $f \mid f^{-1} Y$. Let $f^{1}=f \mid f^{-1} Y$.

Let $\Sigma(f) \in L_{m}^{5}\left(\pi_{1}(Y)\right)$ denote the surgery obstruction associated to the normal cobordism class of $\left(f^{1}, b\right)$. We call $\Sigma(f)$ the abstract surgery obstruction of $f$. Then it can be shown ([Br 2], [Br 3], [Wa 3]) that for problems of codimension $(q) \geqslant 3$ the vanishing of $\Sigma(f)$ is a necessary and sufficient condition for the splittability of $f$. (It is also necessary to assume $\operatorname{dim} V \geqslant 5$.) Furthermore, (1) in such codimensions a Poincaré embedding of PL (resp., TOP) manifolds is always induced by a unique (up to isotopy) locally-flat PL (resp., TOP) embedding. (The smooth case is a bit trickier. See [Br 2], [Wa 3].) (2) Any homotopy equivalence $F: M^{M} \rightarrow V^{m+q}$ is homotopic to an embedding provided $M, V$ are compact and $q \geqslant 3, m+q \geqslant 5$.

As far as 4-dimensional topology go these results give no new information about embedding 1-manifolds into 4-manifolds. All such results are easily handled by general position arguments. One can however get some useful information about embeddings of 4-manifolds into manifolds of higher dimension. Clearly $M^{4} \rightarrow V^{8}$ is always homotopic to an embedding by a general position argument. Thus the only cases of interest are questions about maps $M^{\mathbf{4}} \rightarrow V^{7}$. Interesting theorems in that direction are then the following results of Hirsh [Hi 3] and Boéchat and Haefliger [BH]. (Both results can actually be proven entirely without the use of surgery theory.)

THEOREM 7.12 [Hi 3]. Let $M^{4}$ be a compact, connected, unbounded orientable PL 4-manifold. Then there is a (locally-flat) PL embedding of $M^{4}$ into $S^{7}$. (Note that there exist locally-flat PL embedding of $M^{4}$ into $S^{7}$ which are not concordant to smooth embeddings [Hi 3], [Hi 4], [BH]).

THEOREM 7.13 [BH]. Let $M^{4}$ be a compact, connected, unbounded orientable smooth 4-manifold.

Then $M^{4}$ smoothly embeds in $S^{7}$ if and only if there exists a class $W \in$ $H_{2}(M ; Z)$ such that $W$ is characteristic and $W^{2}=\sigma(M)$. (It is not known whether such $a W$ always exists or not. One can always find a characteristic element in $H_{2}(M ; Z)$ and for any characteristic element $W, W^{2} \equiv \sigma(M)$ $(\bmod 8)$.

We now turn to codimension $q<3$. As the codimension 2 situation is the most complicated we first consider $q=1$ and then turn our attention to $q=2$. The simplest form of the codimension 1 splitting problem can be phrased as follows

Suppose $W$ is homotopy equivalent to a nontrivial connected sum, is $W$ itself a nontrivial connected sum. (We say $W$ is a nontrivial connected sum if $W=M \# N$, with $M$ and $N$ not homotopy spheres.)

Clearly this is just the special case, $Y=S^{n-1}$, of the codimension 1 
splitting question which asks: If $\bar{f}: W^{n} \rightarrow X^{n}$ is a (simple) homotopy equivalence and $Y^{n-1} \subset X^{n}$ is a submanifold (to which we can assume without loss of generality $f$ is transverse) then is $\bar{f}$ homotopic to a map $f$ with $f \mid f^{-1}(Y)$ : $f^{-1}(Y) \rightarrow Y$ a (simple) homotopy equivalence.

For the connected sum question one has the following results in dimension $\neq 4$.

THeOREM 7.14. Let $n \neq 4$ and suppose $W, M, N$ are closed connected $n$ manifolds.

Suppose $W^{n}$ is homotopy equivalent to the nontrivial connected sum $M \# N$. If neither $\pi_{1}(M)$ nor $\pi_{1}(N)$ have elements of order 2 then $W^{n}$ is itself a nontrivial connected sum $M^{\prime} \# N^{\prime}$ with $\left(M^{\prime}, N^{\prime}\right)$ homotopy equivalent to $(M, N)$.

(The cases $n \geqslant 5$ will follow from a theorem of Cappell [Cap 1] which we will quote as Theorem 7.17. The case $n=3$ is true with no conditions on $\pi_{1}(M)$ and $\pi_{1}(Q)$ by a result of Stallings [St 2] while for $n \leqslant 2$ they follow from the classification of 1- and 2-manifolds.)

Even if $n \neq 4$ some condition on $\pi_{1}(P), \pi_{1}(Q)$ is necessary as the following theorem of Cappell [Cap 2] shows.

THEOREM 7.15. There is a closed differentiable $4 k+1$-manifold $W$, simple homotopy equivalent to $\mathbf{R} P^{4 k+1}$ \# $\mathbf{R} P^{4 k+1}, k \geqslant 1$, which is not as a differentiable, PL or even as a topological manifold a nontrivial connected sum. (It is however normally cobordant to $\mathbf{R} P^{4 k+1} \# \mathbf{R} P^{4 k+1}$.)

The proofs of Theorems 7.14 and 7.15 depend (if $n \geqslant 5$ in Theorem 7.14) on high-dimensional surgery techniques and have no analogues in dimension 4. Notice that the extension of Theorem 7.14 to dimension 4 would imply that all simply-connected 4-manifolds $M$ with indefinite intersections forms of type I can be completely decomposed as $M=k \tilde{P} \# l \tilde{Q}$ where $\tilde{P}$ is homotopy equivalent to $P$ and $\tilde{Q}$ is homotopy equivalent to $Q$. (This would show that the conjectures of [MM 1] fail) and would, of course, also show that all simply-connected 4-manifolds $M$ are $A C D$ (actually either $M$ or $(-M)$ would be $A C D$ ).

Theorem 7.14 is a consequence of the general codimension 1 splitting theorem of Cappell [Cap 1]. Cappell's theorem gives very general conditions on when a homotopy equivalences splits along a codimension 1 submanifold.

In particular, one has:

THEOREM 7.16. Let $n \geqslant 5$ and $H=$ DIFF, TOP or PL. Suppose $X$ is $a$ closed $H$-manifold of dimension $n+1$ with $\pi_{1}(X)=G$ and suppose $Y$ is a closed codimension 1 submanifold with trivial normal bundle, $\pi_{1}(Y)=K$, such that $K$ is square-root closed in $G$.

Suppose $X-Y$ has two components (resp., one component) with fundamental groups $G_{i}, i=1,2$ (resp., group $J$ ).

Then if the natural map $\mathrm{Wh}\left(G_{1}\right) \oplus \mathrm{Wh}\left(G_{2}\right) \rightarrow \mathrm{Wh}(G)$ (resp. $\mathrm{Wh}(J) \rightarrow$ $\mathrm{Wh}(G))$ is surjective every homotopy equivalence $f: W \rightarrow X$ is splittable. 
Note. Actually if $n$ is even, one need only assume that the image of the torsion of $f, \mathcal{T}(f) \in \mathrm{Wh}(G)$, is zero in $\mathrm{Wh}(G) / \mathrm{Wh}\left(G_{1}\right) \oplus \mathrm{Wh}\left(G_{2}\right)$ (resp., $\mathrm{Wh}(G) / \mathrm{Wh}(J))$. For $n$ odd an additional invariant of the homotopy class of $f, \theta(f)$ must vanish. For all $n \geqslant 5$ if $f$ is a simple homotopy equivalence it is splittable. (A general discussion of what happens in the more general case when $K$ is not-square-root closed can be found in [Cap 4], [Cap 5].)

TheOREM 7.17. Let $H=$ DIFF, TOP or PL.

Let $f: W^{n+1} \rightarrow X^{n+1}$ be a homotopy equivalence with $W, X$ closed $H$-manifolds. Suppose $Y^{n}$ is a simply-connected closed submanifold of $X^{n+1}$.

Assume also $n \geqslant 4$ and either $\pi_{1}(X)$ has no elements of order 2 or one of the components of $X-Y$ is simply connected. Then $f$ is splittable along $X$.

Notice that Theorem 7.17 (as opposed to Theorem 7.16) does give information about splitting homotopy equivalences along codimension 1 manifolds $Y^{4}$ of dimension 4. Also the cases $n \geqslant 5$ of Theorem 7.14 are special cases of the corresponding results in Theorem 7.17.

Actually Theorem 7.16 (and the note succeeding it) is extendable to the case when $\operatorname{dim} Y=4$ as well.

There are two alternative approaches. One can either emulate Theorem 6.4 and add the conditions that $H_{2}\left(\pi_{1}(Y) ; Z_{2}\right)=L_{5}\left(\pi_{1}(Y)\right)=0$ and obtain the same conclusions as in Theorem 7.17 or settle for a stable splitting result. (That this was bound to occur follows from our general discussion of the problems of 4-dimensional surgery in Chapter 6.)

We state these results as:

THEOREM 7.18. (1) Theorem 7.16 (and the note succeeding it) is also true if $n=4$ provided $L_{5}\left(\pi_{1}\left(Y^{4}\right)\right)=0$ and $H_{2}\left(\pi_{1}(Y) ; Z_{2}\right)=0$ [Cap 1].

(2) In general if $n=4$ in Theorem 7.16 then the hypotheses of Theorem 7.16 (and the succeeding note) guarantee that $f$ is splittable along a submanifold $Y^{\prime}$ isomorphic to $Y \# k\left(S^{2} \times S^{2}\right)$, for some $k \geqslant 0$, obtained by adding on copies of $S^{2} \times S^{2}$ to $Y$, each $S^{2} \times S^{2}$ lying in some neighborhood $\Delta \times I \subset X, \Delta a$ 4-simplex of $Y$. By a small homotopy of $f$ it can always be assumed that $f\left|f^{-1}\left(Y^{\prime}\right)=f\right| f^{-1}(Y) \# k\left(\operatorname{id}_{S^{2} \times s^{2}}\right)[$ CS 1].

The foregoing show that one can therefore handle problems associated with 4-dimensional submanifolds of 5-manifolds in a large number of cases.

What about extensions to $n=3$, that is questions about codimension 1 submanifolds of 4-manifolds?

Here almost nothing is known! At the present time we have no general techniques which handle such problems!

The extent of our lack of knowledge about problems of 3-manifolds embedded in 4-manifolds is amply illustrated by our failure to prove the 4-dimensional PL Schoenflies conjecture!

We recall

PL Schoenflies Conjecture (SC $(n)$ ). Let $S^{n-1}$ be a PL-embedded submanifold of $S^{n}$. Then $S^{n}-S^{n-1}$ consists of two components each of which has closure a PL $n$-disc. 
Remarks. By duality theory $S^{n}-S^{n-1}$ always has exactly two components. If $n=1, \operatorname{SC}(1)$ is of course trivial. If $n=2, \operatorname{SC}(2)$ is true even if we assume $S^{1}$ is only topologically embedded. In fact $\mathrm{SC}(2)$ follows readily from the Jordan curve theorem for $S^{2}$. (Note that $\mathrm{SC}(2)$ immediately implies the 2-dimensional annulus conjecture!)

If $n=3$ then the PL hypothesis is, of course, necessary, as shown by the existence of the Alexander and Antoine horned spheres! (See [RIf] for an entertaining exposition.) Under the hypothesis stated above $\mathrm{SC}(3)$ was proven by Alexander in 1924 [Ax 2].

If $n=4$ then SC(4) is still open.

One can separate the question inherent in $\mathrm{SC}(n)$ into two questions (see [RS, p. 47]).

Suppose $S^{n-1} \subset S^{n}$ as above and $T_{n}$ is the closure of some component.

Then

$\left(1_{n}\right)$ Is $T_{n}$ a PL-manifold?

(2n) If $T_{n}$ is a PL-manifold is it a PL $n$-disc?

Note that $T_{n}$ is a manifold if and only if the other closure is a manifold [RS, Theorem 3.14]. Furthermore looking at links of a point in $S^{n-1}$ we see that $\left(1_{n}\right)$ is equivalent to $\operatorname{SC}(n-1)$.

To separate $\left(1_{n}\right)$ and $\left(2_{n}\right)$ we recall that a proper embedding $M^{m} \hookrightarrow N^{m+q}$ is locally flat if each point $p \in M$ has a neighborhood $U$ in $N^{m+q}$ such that $(N, M) \cap U$ is isomorphic to $\left(D^{m} \times D^{q}, D^{m} \times 0\right)$.

Then in $\mathrm{SC}(n)$, the embedding of $S^{n-1} \hookrightarrow S^{n}$ is PL locally flat if and only if $T_{n}$ is a PL-manifold!

We thus divide $\mathrm{SC}(n)$ into two statements:

$\mathrm{SC}(n)_{1}$. Every PL embedding of $S^{n-1}$ into $S^{n}$ is PL locally flat. [This is, as we noted previously, equivalent to $\operatorname{SC}(n-1)$.]

$\mathrm{SC}(n)_{2}$. If $S^{n-1} \hookrightarrow S^{n}$ is PL locally flat then $T_{n}$ is a PL disc.

If $n \neq 4$ the answer to $\operatorname{SC}(n)_{2}$ is always positive. That is:

THEOREM 7.19. Let $S^{n-1} \hookrightarrow S^{n}$ be a PL locally-flat embedded submanifold. Then if $n \neq 4, T_{n}$ is a PL $n$-disc.

PRoof. If $n \geqslant 3$ this of course follows from the truth of $\operatorname{SC}(n)$ for those cases.

In $n \geqslant 5$ we use the generalized (PL) Poincare theorem in dimension $\geqslant 5$ to deduce that since $T \cup_{\partial} B^{n}$ is a homotopy sphere, $T$ must then be an $n$-ball.

Note then the truth of $\mathrm{SC}(n)_{2}$ for $n=4$ would imply that $\mathrm{SC}(n)$ is true for all $n$.

As it is we do not even know if the closures of the components of $S^{5}-i\left(S^{4}\right)$ are manifolds if $i$ is not locally flat!

We do have the following results though if we either strengthen our hypotheses or weaken our expectations.

TheOREM 7.20 (SEe [RS, p. 47], [Bron]). Let $S^{n-1} \hookrightarrow S^{n}$ be a TOP locallyflat embedded submanifold ( $n$ arbitrary). Then $T_{n}$ is a topological $n$-ball.

Note that Theorem 7.20 implies the weak annulus theorem in all dimensions. That is: 
THEOREM 7.21 (WEAK ANNULUS THEOREM). Let $K_{1}, K_{2}$ be disjoint locally-flat $(n-1)$-spheres topologically embedded in $S^{n}$. Let $U$ denote the open region between them. Then $U$ is homeomorphic to $S^{n-1} \times(0,1)$ and the union of $U$ with either $K_{1}$ or $K_{2}$ is homeomorphic to $S^{n} \times[0,1)$.

(As we mentioned previously the annulus theorem of Kirby [Kirb 4] asserts that if $n \neq 4$ then the closure of $U$ is homeomorphic to $S^{n-1} \times[0,1]$. This is still unknown if $n=4$. If our $K_{i}$ are PL then [RS §3] $U$ is in fact PL-homeomorphic to $S^{n-1} \times[0,1]$. See [Kirb 4] for a complete discussion and further references.)

We now consider the codimension 2 situation. Here the possibilities are more exciting since for 4-dimensional manifolds $M^{4}$ there are many unanswered questions both about maps $F^{2} \rightarrow M^{4}$ (as we saw in Chapter 6) and about maps $M^{4} \rightarrow V^{6}$.

The techniques for studying codimension 2 splitting and embedding questions are due to Cappell and Shaneson and developed in the series of papers [CS 5], [CS 6], [CS 7], [CS 8], [CS 9], [CS 10], [CS 11]. The basic techniques used are (1) a subtle generalization of surgery theory to the problem of finding homology equivalences, and (2) a new classifying space for codimension 2 regular neighborhoods. We refer the reader to the above papers for details. For our use we extract the following theorems.

THEOREM 7.22 [CS 5, ChAPTER 3]. Suppose $f: M^{n} \rightarrow X^{n}$ and $Y^{m} \subset X^{n}$ are as in the discussion following Definition (PE) and let $\Sigma(f)$ be the abstract surgery obstruction of $f$ as defined there.

Then if $n-m=2, m \geqslant 5$ and $n$ is odd, $f$ is (simply) splittable if and only if $\Sigma(f)$ is zero.

In the even case the corresponding theorem is false unless additional restrictions are put on $f$. As we are primarily interested in dimension 4 manifolds we are forced to consider these added technical difficulties as well.

As we mentioned above, Cappell and Shaneson in [CS 5] introduced functors $\Gamma_{n}$, analogous to Wall's $L_{n}$, which measure the obstruction to a map being a homology equivalence. More precisely corresponding to any ring homomorphism $\mathscr{F}: \mathbf{Z}\left[\pi_{1}\right] \rightarrow \Lambda$ they produce functors $\Gamma_{n}[\mathscr{F}]$ to abelian groups, which are periodic of order 4 , and a map of pointed sets $\mathscr{T}_{n}(X) \stackrel{\sigma}{\rightarrow} \Gamma_{n}[\mathscr{F}]$ such that $(f, b): M \rightarrow X$ is normally cobordant to a (simple) $\Lambda$-homology equivalence if and only if $\sigma(f, b)=0$. Then we have:

THEOREM 7.23 [CS 5]. Suppose in 7.22 above we have $m \geqslant 4$ and $n$ even.

Then $f$ is (simply) splittable if and only if $\Sigma(f)=0$ and a well-defined obstruction in a factor group $\Gamma_{n+1}(\phi)$ vanishes. In particular if $\pi_{1}(X-Y)=\mathbf{Z}$ or 0 and $n \geqslant 8, f$ is (simply) splittable if and only if $\Sigma(f)=0$.

Theorem 7.24 [CS 8]. Let $\theta$ be an oriented Poincaré embedding of $M^{n}$ in $W^{n+2}, M$ a closed PL-manifold, $W$ a connected compact PL-manifold and $n \geqslant 3$. If $n$ is even suppose $\pi_{1}\left(E_{\theta}\right) \stackrel{\left(h_{\theta}^{-1}\right)}{\rightarrow} \pi_{1}(W)$ is an isomorphism or $\pi_{1}(W)=$ 0 and $\pi_{1}\left(E_{\theta}\right)$ cyclic.

Then there exists a PL embedding $f$ of $M$ in $W$ realizing $\theta$ ( $f$ is not necessarily locally flat). 
THEOREM 7.25 [CS 6], [CS 8]. Let $f: M^{n} \rightarrow W^{n+2}$ be a homotopy equivalence, $M$ a closed PL-manifold, $W$ a compact connected PL-manifold, $n \geqslant 3$ with $w_{1}(M)=f^{*} w_{1}(W)$. If $n$ is even suppose either that (1) $M, W$ are oriented, $H_{2}\left(\pi_{1}(W) ; Z\right)=0$ and $\chi(W)$, the Poincare dual of $f_{*}[M] \in H_{n}(W)$, is a primitive generator of $H^{2}(W) \approx H^{2}(M)$ or (2) that $\pi_{1}(W)=0$.

Then $f$ is homotopic to a PL-embedding.

Corollary 7.26. Let $M^{n}$ be a closed connected oriented PL-manifold with $n \geqslant 3$. Suppose that the Hurewicz homomorphism $\pi_{n+1}(\Sigma M) \rightarrow H_{n+1}(\Sigma M)$ is surjective. Then there exists a PL-embedding of $M$ in $S^{n+2}$.

Note that the hypotheses of Corollary 7.26 are satisfied by any spin 4-manifold.

We have in actuality:

COROLlARY 7.27 [CS 11]. Let $M^{4}$ be a closed connected oriented PL or TOP 4-manifold then

(1) There exists a PL (TOP) embedding of $M$ into $S^{6}$ if and only if $w_{2}\left(M^{4}\right)=0$.

The embedding can be chosen to have only one non-locally-flat point $x$ which is the vertex of a cone on a knot $K \subset S^{5}$ with $\sigma\left(S^{5}, K\right)=\sigma(M)$.

(2) There exists a locally-flat PL (TOP) embedding of $M$ into $S^{6}$ if and only if $w_{2}\left(M^{4}\right)=\sigma\left(M^{4}\right)=0$.

One can actually get results corresponding to Theorems 7.25 and Corollary 7.26 above for homotoping $f$ to an immersion as well. For dimension 4 manifolds one obtains

Proposition 7.28. Let $M^{4}$ be as in Corollary 7.27 above. Then

(1) There exists a PL (TOP) immersion of $M^{4}$ into $S^{6}$ if and only if $\chi(M)$ is even [CS 11].

(2) There exists a smooth (PL or TOP locally-flat) immersion of $M^{4}$ into $S^{6}$ if and only if there exists $X \in H^{2}(M)$ with $X$ characteristic and $X^{2}=3 \sigma(M)$ [Hi 5].

We assert that if $n$ is even, some assumptions on $\pi_{1}\left(M^{n}\right)$ in Theorems 7.25 and 7.26 are indeed necessary. Recall that if $W^{m}$ is a compact PL-manifold with boundary then a spine of $W$ is a PL embedding $\phi: M^{n} \rightarrow W^{m}, M$ a closed PL-manifold, that is a homotopy equivalence.

Then in [CS 9], [CS 10] Cappell and Shaneson proved:

THEOREM 7.29. Let $n \geqslant 4$ be even.

Suppose $M^{n}$ is a closed PL-manifold such that $\pi_{1}\left(M^{n}\right)$ is finite and has a central subgroup with nontrivial abelian quotient. Then there are infinitely many manifolds $W$ simple homotopy equivalent to $M$ such that any PL-map $\phi:$ $M^{n} \rightarrow W^{n+2}$ which is a simple homotopy equivalence is not homotopic to a PL-embedding. $\phi$ will however always be homotopic to a PL-immersion.

Thus there exist totally spineless manifolds in even dimensions greater than 4. In particular our assertion concerning the necessity of restriction on $\pi_{1}\left(M^{2 k}\right)$ in Theorems 7.25 and 7.26 follows from Theorem 7.29. 
All of the above theorems have assumed that $n \geqslant 3$ or $n \geqslant 4$. Thus we can assert that the theory of maps of $M^{4} \rightarrow W^{6}$ is completely explained by high-dimensional surgery-type techniques and does not differ from the general theory of maps $M^{2 n} \rightarrow M^{2 n+2}$.

The methods used in establishing Theorems 7.23 through 7.29 also give a great deal of information on high-dimensional knotting phenomena and the high-dimensional knot cobordism groups. (See [CS 5], [CS 13].) Essentially what is of relevance to us is that knottings of $S^{4}$ in $S^{6}$ are also higher-dimensional phenomena with an associated theory entirely equivalent to the general theory of knottings of $S^{2 m} \hookrightarrow S^{2 m+2}$. This is however not precisely true for knottings of $S^{2} \hookrightarrow S^{4}$. We discuss these in greater detail in the next section.

It is also clear by our discussion in $\$ 1$ and $\$ 2$ that the analogues of Theorems 7.22 through 7.29 are not true for maps $F^{2} \hookrightarrow M^{4}$. An adequate theory to explain the possible phenomena associated with such maps is not yet available! Not surprisingly, however, the counterexamples of Theorem 7.24 can be extended to lower dimensions. In [Mt 2], Matsumoto has shown that 4-dimensional manifolds also need not necessarily have spines. More particularly we have:

THEOREM 7.30 [Mt 2]. There exists a compact 4-dimensional PL-manifold $W^{4}$ with boundary such that

(1) $W^{4}$ is homotopy equivalent to $T^{2}$.

(2) No homotopy equivalence $T^{2} \rightarrow W^{4}$ is homotopic to a PL-embedding.

As Matsumoto's construction is so pretty we sketch it here.

Construction of $W^{4}$. Let $h: S^{1} \rightarrow S^{1} \times D^{2}$ be the embedding indicated in Figure 7.7. Essentially the same embedding $S^{1} \rightarrow S^{1} \times S^{2}$ was used by Mazur [Mz] to construct a contractible 4-manifold. Extend $h$ to a framed embedding $\bar{h}: S^{1} \times D^{2} \rightarrow S^{1} \times D^{2}$ in such a way that $\bar{h}$ followed by the natural inclusion $S^{1} \times D^{2} \rightarrow S^{3}$ is isotopic to a trivial knot with a trivial framing. Our manifold $W^{4}$ is the mapping torus of the framed embedding $\bar{h}$. More precisely, $W^{4}$ is obtained from a product $S^{1} \times D^{2} \times[0,1]$ by identifying $(x, \xi) \times\{1\}$ with $\bar{h}(x, \xi) \times\{0\}$ for each $(x, \xi) \in S^{1} \times D^{2}$. Since $h$ is homotopic to the zero cross section $S^{1} \times\{0\} \rightarrow S^{1} \times D^{2}, W^{4}$ is homotopically equivalent to $T^{2}$.

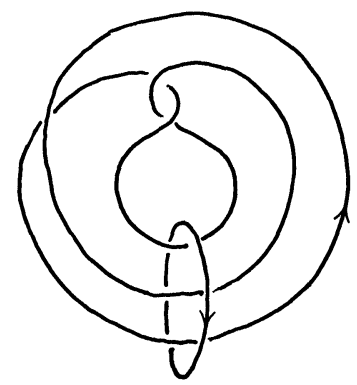

Figure 7.7 Mazur's embedding. 
For a proof of assertion 2 in Theorem 7.23 we refer the reader to [Mt 2]. We recall that Mazur's construction [Mz], [Z 4], [AK 2] has had a long history of furnishing raw material for the construction of counterexamples or possible counterexamples in 4-dimensional topology. For further discussions of

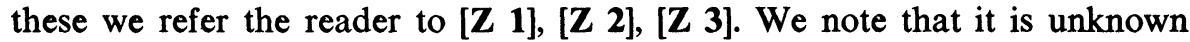
whether a similar example exists with $T^{2}$ replaced by $S^{2}$.

7.4 Codimension two phenomena: Knotting. In the previous section we mentioned that the knot theory of embeddings of $S^{4}$ in $S^{6}$ is entirely analogous to embeddings of $S^{2 m}$ in $S^{2 m+2}$ for $m \geqslant 3$ and displays no intrinsically 4-dimensional phenomena. This is not completely true about knottings of $S^{2}$ in $S^{4}$. Although most of the properties that such knots enjoy are also characteristic of higher-dimensional knots as well, the fact that the 4-dimensional Poincaré conjecture is still unsettled does lead to some complications!

We recall that $K$ is a knot in $S^{n}$ if and only if $K$ is the image of an embedding $i: S^{n-2} \hookrightarrow S^{n}$. For simplicity we shall assume that $i$ is always a PL-locally-flat map. (Thus all our knots will be tame in the sense of [Fox].) We say two knots $K_{1}, K_{2}$ in $S^{n}$ are equivalent if there is a (PL)-homeomorphism $h: S^{n} \rightarrow S^{n}$ with $h\left(K_{1}\right)=K_{2}$. The equivalence class of a knot is called its knot-type. The equivalence class of the standard embedding of $S^{n-2} \hookrightarrow S^{n}$ is called the trivial knot-type.

Generally most attempts to study the knot-type of a given knot $K$ are based on studying algebraic invariants of the knot complement, $S^{n}-K$. For example, if $K$ is a knot in $S^{3}$ then $K$ has the trivial knot-type if and only if $\pi_{1}\left(S^{3}-K\right)=\mathbf{Z}$ ([RIf, Chapter 4]). However if $n>5$, then there exist infinitely many inequivalent knots $K$ in $S^{n}$ with $\pi_{1}\left(S^{n}-K\right)=\mathbf{Z}$ and $K$ not equivalent to the trivial knot [RIf, Chapter 7]. Furthermore even if $n=3$ there exist inequivalent knots $K_{1}, K_{2}$ in $S^{3}$ with $\pi_{1}\left(S^{3}-K_{1}\right)$ isomorphic to $\pi_{1}\left(S^{3}-K_{2}\right)$. For example the square knot and granny knot (see Figure 7.8) are inequivalent [RIf] but their complements are homotopy equivalent [RIf].

We are thus led to inquire into the extent to which the knot complement determines the knot-type. More specifically suppose $K$, is a knot in $S^{n}$. If $T(K)$ is a tubular neighborhood of $K$ with interior $\dot{T}(K)$, then $S^{n}-\dot{T}(K)$ is called the exterior of the knot $K$ and denoted by $X(K)$. It is a compact manifold with boundary $K \times S^{1}$ and its interior is isomorphic to the knot complement $S^{n}-K$.

Now suppose $K_{1}, K_{2}$ are knots in $S^{n}$. As the example of the square and granny knots shows it is possible for inequivalent knots to have homotopy equivalent complements. However if we examine the exteriors of the square and granny knots it is possible to show (see [RIf]) that there does not exist any homotopy equivalence rel $\partial$ between them. (Equivalently no homotopy equivalence of the exteriors preserves peripheral structure in the sense of [Hemp].) We are thus led to ask:

(1) Suppose $K_{1}, K_{2}$ are knots in $S^{n}$ with $X\left(K_{1}\right)$ homotopy equivalent rel $\partial$ to $X\left(K_{2}\right)$. Is $K_{1}$ then equivalent to $K_{2}$ ?

In the case of classical knots of $S^{1}$ in $S^{3}$, Waldhausen [Wald] has shown that if $X\left(K_{1}\right)$ is homotopy equivalent rel $\partial$ to $X\left(K_{2}\right)$ then they are homeomorphic. One thus breaks up question (1) into two questions as follows: 
Let $K_{1}, K_{2}$ be knots in $S^{n}$. then

(1A) Does $X\left(K_{1}\right)$ is homotopy equivalent to $X\left(K_{2}\right)$ rel $\partial$ imply that $X\left(K_{1}\right)$ is homeomorphic to $X\left(K_{2}\right)$ ?

(1B) Does $X\left(K_{1}\right)$ is homeomorphic to $X\left(K_{2}\right)$ imply that $K_{1}$ is equivalent to $\mathrm{K}_{2}$ ?

A negative answer to (1A) was obtained by Cappell in [Cap 3]. In particular he exhibits an infinite number of pairs of inequivalent knots $K_{i}, K_{i}^{\prime}$ in $S^{7}$ such that $X\left(K_{i}\right)$ is simple homotopy equivalent rel $\partial$ to $X\left(K_{i}^{\prime}\right)$ but $X\left(K_{i}\right)$ is not homeomorphic to $X\left(K_{i}^{\prime}\right)$.

$$
\pi_{1}\left(S^{3}-K_{2}\right)=G_{2}=\langle x, y, z: x y x=y x y, x z x=z x z\rangle=G_{1} \text {. }
$$

FIGURE 7.8

(The knots $K_{i}$ constructed by Cappell are obtained via a process of '3-super-spinning' a knot pair $\left(\Sigma^{4}, K_{i}\right)$, where $\Sigma^{4}$ is a homotopy 4-sphere. Thus $\pi_{1}\left(S^{7}-K_{i}\right)=\pi_{1}\left(\Sigma^{4}-K_{i}\right)$ and so the $K_{i}$ are the 'knot groups' of some knotted $S^{2}$. Quinn has shown that if $n \geqslant 6$ and $\pi$ is the 'knot group' of a classical knot $S^{1} \hookrightarrow S^{3}$ and $\pi_{1}\left(S^{n}-K_{1}\right)=\pi_{1}\left(S^{n}-K_{2}\right)=\pi$ then $X\left(K_{1}\right)$ is homotopy equivalent rel $\partial$ to $X\left(K_{2}\right)$ implies $X\left(K_{1}\right)$ is homeomorphic to $X\left(K_{2}\right)$.)

Returning to Question (1B), we note that in [Glk] Gluck showed that if $n=4$ there could be at most two inequivalent knots $K, K^{\prime}$ in $S^{n}$ with homeomorphic knot complements. In [Kato], [Br 4], [LS 2] this result was extended to all $n \geqslant 5$. The actual realization of two inequivalent knots with homeomorphic (in fact diffeomorphic) complements is given in [CS 12]. The Cappell and Shaneson construction goes as follows.

Let $A \in \mathrm{SL}(n+1 ; \mathrm{Z})$ be such that

(i) if $A^{(r)} \in \mathrm{GL}\left(\left(\begin{array}{c}n+p \\ r\end{array}\right)\right.$; Z) is the $r$-fold exterior product of $A$ with itself then $\left|\operatorname{det}\left(I-A^{(r)}\right)\right|=1$ for $1<r \leqslant n, n \geqslant 2$, and

(ii) $\operatorname{det}(A-\lambda I)>0$ for $\lambda<0$. 
Let $\phi(A)$ be the obvious diffeomorphism of $T^{n+1}=R^{n+1} / Z^{n+1}$ inducing $A$ on $\pi_{1} T^{n+1} \simeq Z^{n+1}$ and denote the mapping torus of $\phi(A)$ by $M_{A} \cdot\left(M_{A}=\right.$ $\left(T^{n+1} \times I\right) /(x, 0) \sim(\phi(A)(x), 1)$.) Denote the image of the origin in $T^{n+1}$ by $\theta$ and let the embedded circle $C \subset M_{A}$ be the image of $\theta \times I$ in $M_{A}$.

Now let $D^{n+1} \times C \subset M_{A}$ be a tubular neighborhood of $C=0 \times C$. Let $N_{A}=M_{A}-\left(D^{n+1} \times C\right)$ with $\partial N_{A}=D^{n+1} \times C$. Suppose $\mu$ is the nontrivial bundle map of $D^{n+1} \times C$ corresponding to the nonzero element of $\pi_{1}(\mathrm{SO}(n$ $+1)$ ) and let $\bar{\mu}=\mu \mid \partial D^{n+1} \times C$. Write $C=\partial D^{2}$.

Set $\Sigma_{0}=N_{A} \cup \partial D^{n+1} \times D^{2}$ and let $\Sigma_{1}=N_{A} \cup_{\bar{\mu}} \partial D^{n+1} \times C$ (i.e. identify $x \in \partial N_{A}$ with $\bar{\mu}(x) \in \partial D^{n+1} \times C$ to obtain $\Sigma_{1}$ ). Then by [CS 12, Proposition 1.B] both $\Sigma_{0}$ and $\Sigma_{1}$ are smooth homotopy $(n+2)$-spheres.

Let $K_{i} \subset \Sigma_{i}$ be the smoothly-embedded $n$-sphere

$$
\partial D^{n+1}=\partial D^{n+1} \times 0 \subset \partial D^{n+1} \times D^{2} \subset \Sigma_{i} \text {. }
$$

Then Cappell and Shaneson show [CS 12, Proposition 3] that there is no diffeomorphism from $\Sigma_{0}$ to $\Sigma_{1}$ that throws $K_{0}$ onto $K_{1}$. To actually produce inequivalent knots with the same complement it suffices to show that $A \in$ $\operatorname{SL}(n+1 ; \mathbf{Z})$ with the desired properties actually exist. This is done for $n=2,3,4$ in [CS 12]. (It is reasonable to suppose that such $A$ actually exist for all $n \geqslant 2$, but in addition to the cases $n=2,3,4$ exhibited in [CS 12] this has, at present, only been verified for $n=5$.) In fact an infinite family of acceptable matrices $A$ is produced. If $n \geqslant 3$ the truth of the Poincare conjecture in dimension larger than 4 shows that $\Sigma_{i}$ is PL-homeomorphic to $S^{n+2}$ and thus provides us with:

THEOREM 7.31 [CS 12]. For $n=3,4$, there are infinitely many pairs of inequivalent spherical $n$-knots in $S^{n+2}$ such that each pair consists of two inequivalent knots with diffeomorphic exteriors. (The pairs are distinguished by their first Alexander polynomials.)

[A knot $K \in S^{n+2}$ is called spherical if $K$ is smoothly embedded in $S^{n+2}$ and diffeomorphic (rather than just PL-homeomorphic) to $S^{n}$.]

The above method does not extend directly to 2 -knots in $S^{4}$. However using a somewhat different construction Gordon [Gor 2] manages to do just that! Thus suppose $K$ is a knot in $S^{n}, n \geqslant 4$. Let $D^{2} \times K$ be a tubular neighborhood of $K$ in $S^{n}$ and set $X=S^{n}-D^{2} \times K$. Let $\bar{\mu}$ be the restriction of the nontrivial bundle map of $S^{1} \times D^{n-1}$ to $S^{1} \times K=\partial X$. Then set $\Sigma(K)=X \cup_{\bar{\mu}} D^{2} \times S^{n-2}$ and $K^{*}=0 \times S^{n-2} \subset \Sigma(K)$. Gordon then proves [Gor 2]:

THEOREM 7.32. Let $K$ be a knot of $S^{1}$ in $S^{3}$. Let $l$ be a positive integer and let $K^{(l)}$ be the l-twist spin of $K$ in the sense of $[\mathbf{Z} 4]$.

Then $\Sigma\left(K^{(l)}\right)$ is homeomorphic to $S^{4}$.

Lemma 7.33. Let $K$ be a knot of $S^{n-2}$ in $S^{n}, n \geqslant 3$, and suppose $l$ is an odd integer. Let $K^{(l)}$ be the l-twist spin of $K$. If the universal cover of the l-fold branched cyclic cover associated to $K$ is $R^{n}$, then the knots $K^{(l)}, K^{(l)^{*}}$ are inequivalent.

In particular one notes that if $K$ is a $(p, q)$ torus-knot with $1 / l+1 / p+$ $1 / q \leqslant 1$ then the universal cover of the $l$-fold branched cyclic cover 
associated to $K$ is $R^{3}$ [Gor 2]. (This is also true if $K$ is any nontrivial doubled knot (in the sense of [RIf]) and $l>1$.) Thus Theorem 7.31 extends to knottings of $S^{2}$ in $S^{4}$ and we can state

THEOREM 7.34 (GoRDON, [Gor 2]). There exist infinitely many pairs of inequivalent knots of $S^{2}$ in $S^{4}$ such that each pair consists of inequivalent knots with homeomorphic exteriors.

Having answered both (1A) and (1B) in the negative we can lower our sights and ask:

(2) For which class of knots does (1A) or (1B) hold?

More particularly:

(2') Suppose $K$ is a knot in $S^{n}$ with $X(K)$ homotopy equivalent to $S^{1}$. Does $X(K)$ have the trivial knot-type?

As we pointed out above if $n \geqslant 5$ then there exist knots $K$ in $S^{5}$ with $\pi_{1}\left(S^{5}-K\right)=\mathbf{Z}$ but $K$ not having trivial knot-type. Furthermore by a result of Lashof and Shaneson [LS 2] if $n \geqslant 6$ then $X\left(K_{1}\right)$ homotopy equivalent to $X\left(K_{2}\right)$ and $\pi_{1}\left(X\left(K_{1}\right)\right)=\mathbf{Z}$ implies that $X\left(K_{1}\right)$ is in fact homeomorphic to $X\left(K_{2}\right)$. Thus one can ask:

$\left(2^{\prime \prime}\right)$ Suppose $K_{1}, K_{2}$ are knots in $S^{n}$ with $X\left(K_{1}\right)$ homeomorphic to $X\left(K_{2}\right)$ and $\pi_{1}\left(X\left(K_{1}\right)\right)=\mathbf{Z}$. Is $K_{1}$ equivalent to $K_{2}$ ?

Presently the answer to ( $\left.2^{\prime \prime}\right)$ is unknown, for all $n \geqslant 4$. If $n=3$ or $n \geqslant 5$ then $\left(2^{\prime}\right)$ has a positive answer. If $n=4$ then (as usual) essentially nothing is known. That is we do not know if there exist nontrivial knots $K$ in $S^{4}$ such that $\pi_{1}\left(S^{4}-K\right)=\mathbf{Z}$ or whether $S^{4}-K \approx S^{1}$ implies that $K$ is unknotted!

The proof that (2') has a positive answer if $n \geqslant 5$ goes as follows.

Let $K$ be a knot in $S^{n}$ with exterior $X(K)$. Let $C$ be an $S^{1}$ in the interior of $X(K)$ linking $K$ once and let $N(C)$ be an open tubular neighborhood of $C$ in $X(K)$. Let $W=X(K)-N(C)$. Then $W$ is a cobordism between $C \times \partial D^{n-1}$ $\approx S^{1} \times S^{n-2}$ and $\partial X(K)=S^{1} \times S^{n-2}$. If $X(K)$ has the homotopy type of an $S^{1}$ then a direct calculation shows that $W$ is in fact an $s$-cobordism inducing a diffeomorphism between its ends. Then by the $s$-cobordism theorem if $n \geqslant 6$ or by the results of Shaneson and Wall ([Sh 1], [Wa 3], see also Chapter 6) if $n=5$, we can conclude that $W=S^{1} \times S^{n-2} \times I$ and so $X(K)=S^{1} \times D^{n-1}$. But then $K$ must be unknotted! Notice that if we knew that every $s$-cobordism between $S^{1} \times S^{2}$ and $S^{1} \times S^{2}$ which induced a diffeomorphism on its ends was a product then $\left(2^{\prime}\right)$ would be true for $n=4$ as well. Conversely the failure of $\left(2^{\prime}\right)$ for $n=4$ would imply that the $s$-cobordism theorem is false for 4-dimensional $s$-cobordisms!

Although the theory of knots in $S^{4}$ is not identical to higher-dimensional knot theory it differs even more radically from the theory of classical knots, i.e., $K \subset S^{3}$. Perhaps the most basic results in the theory of classical knots are the unknotting and asphericity theorems whose statements we recall.

Unknotting theorem (PAPAKyriakopoulos). Let $K$ be a knot in $S^{3}$. Then $K$ has the trivial knot-type if and only if $\pi_{1}\left(S^{3}-K\right)=\mathbf{Z}$.

(If $K$ is not $\mathrm{PL}$ then it may be nontrivial and still have $\pi_{1}\left(S^{3}-K\right)=\mathrm{Z}$. See [RIf]. As we mentioned at the beginning of this section all our knots will be PL and thus tame.) 
ASPHERICITY TheOREM (PAPAKYRIAKopoulos). If $K$ is a knot in $S^{3}$, then $\pi_{i}\left(S^{3}-K\right)=0$ for all $i \geqslant 2$.

The unknotting and asphericity theorems were first proved by Papakyriakopoulos in [Papa 1], [Papa 2].

The unknotting theorems is a direct consequence of Dehn's lemma which was proved by Papakyriakopoulos in 1957 [Papa 1], [Papa 2]. (Dehn's lemma was first enunciated by M. Dehn [Dehn] in 1910 but his 'proof' contained serious gaps.) In the same paper in which he proved Dehn's lemma Papakyriakopoulos also stated and proved the sphere theorem which in turn implies that classical knot complements are aspherical.

The statements of Dehn's lemma and the sphere theorem (in its simplest version) are:

DehN's Lemma. Suppose $M^{3}$ is a 3-manifold and $f: D^{2} \rightarrow M^{3}$ is a map of a disc with no singularities on $\partial D^{2}$ (i.e., $x \in \partial D^{2}, x \neq y \in D^{2} \Rightarrow f(x) \neq f(y)$ ). Then there exists an embedding $g: D^{2} \rightarrow M^{3}$ with $g(\partial D)=f(\partial D)$.

SPHere Theorem. Suppose $M$ is an orientable 3-manifold with $\pi_{2}(M) \neq 0$. Then there is a 2-sphere $S$ embedded in $M$ which is not contractible in $M$.

(In addition to [Papa 1], [Papa 2] one can find newer proofs of Dehn's lemma and the sphere theorem in [St 2] and [Hemp].)

In dimension 4 both Dehn's lemma (i.e. replace $M^{3}$ by $M^{4}$ ) and the asphericity of knots are false!

To show that Dehn's lemma is false in dimension 4 it clearly suffices to produce a compact 4-manifold with boundary $M$ and a knot $K$ in $\partial M$ such that $K$ is null-homotopic in $M$ but does not bound an embedded disc (even a nonlocally-flat embedded disc) in $M$. This is done in [AK 3]. In particular if $W_{0}^{4}$ is the manifold with framed link diagram given in Figure 7.3 and $\xi$ is the knot in $\partial W_{0}^{4}$ drawn there, then Akbulut shows that $\xi$ is null homotopic in $W_{0}^{4}$ but does not even bound a nonlocally-flat PL disc embedded in $W_{0}^{4}$ !

We note that $W_{0}^{4}$ is a compact rational homology ball but is not contractible. In [Z 3], Zeeman asked for a compact contractible counterexample to Dehn's lemma in dimension 4. Matsumoto and Venema in [Mt V] give an example of an open subset $W_{0}^{\prime}$ of $\mathbf{R}^{4}$ which is contractible and satisfies $\partial W_{0}^{\prime} \approx S^{1} \times \mathbf{R}^{2}$ but such that no homotopically essential loop on $\partial W_{0}^{\prime}$ bounds a PL-embedded disc in $W_{0}^{\prime}$. However $W_{0}^{\prime}$ is of course not compact and a compact such example is still as yet unknown. If $M^{4}$ is assumed to have special properties, such as $M=N \# S^{2} \times S^{2}$ or $\partial M=\#_{i=1}^{k}\left(S^{1} \times\right.$ $\left.S^{2}\right)_{i}$ then Norman [Nor] showed that the 4-dimensional analogue of Dehn's lemma is true. More generally Fenn [Fenn] showed that if $M$ has a 2-dimensional spine and $\partial M \neq 0$ then any proper map $f: D^{2} \rightarrow M$ is properly homotopic to a locally-flat embedding!

The existence of a knot $K \subset S^{4}$ with $\pi_{2}\left(S^{4}-K\right) \neq 0$ was demonstrated by Andrews and Curtis in [AC]. Their $K$ was a 1-spun trefoil knot. (See [RIf, Chapter 3] for a discussion of spun knots and a presentation of the Andrews and Curtis proof that $\pi_{2}\left(S^{4}-K\right) \neq 0$.)

In [Ep] Epstein showed that if $K$ is any nontrivial spun knot in $S$ then $\pi_{2}(S-K)$ is free abelian of infinite rank. This would suggest that although 
$\pi_{2}$ of a higher-dimensional knot complement is not zero it still might not contain any useful information. Actually as Fox pointed out in [Fox] it is more useful to consider $\pi_{2}\left(S^{4}-K\right)$ as a $\mathbf{Z}\left[\pi_{1}\left(S^{4}-K\right)\right]$-module and calculate its module structure. This was recently accomplished by Lomonaco who in [Lom] gives a procedure for computing $\pi_{2}\left(S^{2}-K\right)$ as a $\mathbf{Z}\left[\pi_{1}\left(S^{4}-K\right)\right]-$ module, for any knot $K \subset S^{4}$. That $\pi_{2}\left(S^{4}-K\right)$ actually gives information about $K$ not already contained in $\pi_{1}\left(S^{4}-K\right)$ was demonstrated by Gordon [Gor 3] who constructed three twist spun knots with the same $\pi_{1}\left(S^{4}-K\right)$ but differing second homotopy modules. It is thus fairly clear that the theory of knots in $S^{4}$ differs radically from knots in $S^{3}$ and is indeed more closely related to higher-dimensional knot theory.

Before leaving the question of knots in $S^{4}$ we consider two other types of questions about knots in $S^{4}$.

(I) We have until now been restricting our attention to PL-knots. In [CS 13] Cappell and Shaneson showed that for each $n ; n \geqslant 5$ there exist infinitely many inequivalent locally-flat TOP-knots $K^{n-2} \subset S^{n}$ which are not topologically equivalent to any PL-knot. Their proof is based on surgery-theoretic results not known in lower dimensions. We can thus ask:

Question. (i) Does there exist a locally-flat topological knot $K^{2} \subset S^{4}$ which is not topologically equivalent to a PL-knot?

(ii) Is there a classical knot $K \subset S^{3}$ which is topologically a slice knot (i.e. bounds a locally-flat topological disc $D^{2} \hookrightarrow D^{4}$ ) but not PL-slice.

(II) Suppose $V$ is an almost completely decomposable 4-manifold. Thus by 'blowing up' a point of $V$ we obtain the decomposable manifold $\tilde{V}=V \# P$. Let $\sigma: \tilde{V} \rightarrow V$ be the 'blowing down' map and set $L=\sigma^{-1}(p)$. Thus $L$ is a smoothly embedded 2 -sphere in $\tilde{V}$. Since $\tilde{V}$ is completely decomposable we can without loss of generality suppose it arose by blowing up $S^{4}$ at a finite set of points $x_{1}, \ldots, x_{k}$. (We use blow up to refer to either $\sigma$ or $\bar{\sigma}$-processes as defined in Chapter 2.) Let $\sigma: \tilde{V} \rightarrow S^{4}$ be the blowing down map. Thus $\phi \mid:$ $\tilde{V}-\phi^{-1}\left\{x_{1}, \ldots, x_{n}\right\} \rightarrow S^{4}-\left\{x_{1}, \ldots, x_{n}\right\}$ is a diffeomorphism and without loss of generality we can suppose that $\phi(L)$ is a smoothly immersed 2 -sphere in $S^{4}$. We thus see that to any almost completely decomposable 4-manifold $V$ we can associate an immersed sphere $K \subset S^{4}$. Conversely given any immersed sphere $K \subset S^{4}$ we can construct a minimal (in some sense) decomposable manifold in which the strict image of $K$ embeds. We are thus led to inquire about the existence of an appropriate equivalence relation on immersed $S^{2}$ s in $S^{4}$ which would correspond to their arising from the same almost completely decomposable 4-manifold. In particular which 'singular knots' in $S^{4}$ correspond to nonsingular hypersurfaces of $\mathbf{C} P^{3}$ and more specifically which 'singular knot' corresponds to $V_{4}$ ?

7.5 Codimension two phenomena. II: THE ZARISKI CONJECTURE. There is an algebraic geometric analogue of the knotting phenomena associated with embeddings of $S^{n} \hookrightarrow S^{n+2}$. Thus suppose $V_{n-1}$ is a projective algebraic hypersurface in $\mathbf{C} P^{n}$. What is $\pi_{1}\left(\mathbf{C} P^{n}-V_{n-1}\right)$ ? What would a complete set of topological invariants be for the embedding of $V_{n-1} \hookrightarrow \mathbf{C} P^{n}$ ? One can motivate this question as follows

Let $a_{1}, \ldots, a_{n}$ be points in $\mathbf{C} P^{1}$ and let $\pi=\pi_{1}\left(\mathbf{C} P^{1}-\left\{a_{1}, \ldots, a_{n}\right\}\right)$. Then Riemann showed that for any homomorphism $\phi: \pi \rightarrow S_{r}$ there exists a 
Riemann surface $V$ and a holomorphic map $f: V \rightarrow \mathbf{C} P^{1}$ which is branched precisely over $a_{1}, \ldots, a_{n}$ such that the monodromy group of $f: V \rightarrow \mathbf{C P} P^{1}$ is precisely $\operatorname{Im} \phi$. Since the structure of $\pi$ above is quite simple one has an attractive vehicle for studying the nature of mappings $f: V \rightarrow \mathbf{C P}$.

Now suppose $V$ is a nonsingular projective algebraic surface. Then as mentioned in Chapter 2 we can find a generic projection of $V$ into $\mathbf{C} P^{3}, \pi$ : $V \rightarrow \mathbf{C} P^{3}$ such that $V^{*}=\pi(V)$ has only ordinary singularities. Furthermore a generic projection $p: V^{*} \rightarrow \mathbf{C} P^{2}$ will have a branch curve $\Gamma$ whose only singularities are nodes and cusps. We can now pose the following two questions.

(1) Suppose $C$ is an algebraic plane curve. What is $\pi_{1}\left(\mathbf{C} P^{2}-C\right)$ ?

(2) Suppose $C$ is an algebraic plane curve with $\pi=\pi_{1}\left(\mathbf{C} P^{2}-C\right)$. Let $\phi$ : $\pi \rightarrow S_{r}$ be a homomorphism. Does there exist a hypersurface $V^{*} \subset \mathbf{C} P^{3}$ with projection $p: V^{*} \rightarrow \mathbf{C} P^{2}$ such that $C$ is the branch curve of $p$ and $V^{*} \stackrel{p}{\rightarrow} \mathbf{C} P^{2}$ has monodromy group $\operatorname{Im} \phi$ ?

Clearly it suffices to study only $C$ such that the singularities of $C$ are only nodes ( $=$ ordinary double points) and cusps.

In [ENR], Enriques answered question (2) in the affirmative and we are left only with question (1). The first results in this direction are:

THEOREM 7.35 [Zar 1], [Zar 3]. Let $V_{n}$ be an irreducible algebraic curve of degree $n$ in $\mathbf{C} P^{2}$.

Then

(1) $\pi_{1}\left(\mathbf{C} P^{2}-V_{n}\right)$ is abelian implies $\pi_{1}\left(\mathbf{C} P^{2}-V_{n}\right) \approx \mathbf{Z} / n \mathbf{Z}$.

(2) $V_{n}$ is nonsingular implies $\pi_{1}\left(\mathbf{C} P^{2}-V_{n}\right)$ is abelian.

It is not true, however, that all irreducible curves have complements with abelian fundamental groups. For example the curve $C:\left(y^{2}-z^{2}\right)^{2}+x^{3}(x-$ $z)=0$ in $\mathbf{C} P^{2}$ is an irreducible quartic with exactly three cusps and no other singularities. Then $\pi_{1}\left(\mathbf{C} P^{2}-C\right)=\left\langle a, b: a^{2}=b^{2}, a^{4}=1,(a b)^{3}=a^{2}\right\rangle$ which is nonabelian. This is the simplest example of a curve whose complement has a nonabelian fundamental group. We in fact have the following theorem.

TheOREM 7.36 [Zar 3]. Let $C$ be a curve of degree $n$ in $\mathbf{C} P^{2}$ having $p$-nodes and q-cusps and no other singularities. Then if $n \leqslant 4$ and $q \leqslant 2$ then $\pi_{1}\left(\mathbf{C} P^{2}-\right.$ C) is abelian.

The example above clearly shows that if one allows cusps one can come up with nonabelian fundamental groups. We thus ask the following question.

(3) Suppose $C$ is an irreducible curve in $\mathbf{C} P^{2}$ whose only singularities are nodes. Is $\pi_{1}\left(\mathbf{C} P^{2}-C\right)$ abelian?

In [Zar 3], Zariski claims to have shown that the answer to question (3) is affirmative. The idea behind Zariski's proof was the following:

Let

$$
F_{t}(x, y, z)=\sum_{i+j+k=n} a_{i j, k}(t) x^{i} y^{j} z^{k}=0
$$

be a family of curves of degree $n$ in $\mathbf{C} P^{2}$ where $t \in[0,1]$ and suppose for $t \neq 0$ that all the $F_{t}$ have the same type of singularities. We shall call $F_{0}$ the limiting curve of the system $F_{t}$. 
Then (i) $t_{1}, t_{2} \neq 0 \Rightarrow F_{t_{1}}$ is ambiently isotopic to $F_{t_{2}}$ and thus $\pi_{1}\left(\mathbf{C} P^{2}-F_{t_{1}}\right)$ $=\pi_{1}\left(\mathbf{C} P^{2}-F_{t_{2}}\right)$.

(ii) In [Zar 3], Zariski shows that if $F_{0}$ has no multiple components then for $t \neq 0, \pi_{1}\left(\mathbf{C} P^{2}-F_{t}\right)$ is a quotient group of $\pi_{1}\left(\mathbf{C} P^{2}-F_{0}\right)$. In particular if $\pi_{1}\left(\mathbf{C} P^{2}-F_{0}\right)$ is abelian then so is $\pi_{1}\left(\mathbf{C} P^{2}-F_{0}\right)$.

(iii) Let $K_{n}$ be $n$ lines in general position in $\mathbf{C} P^{2}$. Then $\pi_{1}\left(\mathbf{C} P^{2}-K_{n}\right)$ is abelian [Zar 3].

As a consequence of (i)-(iii) one sees that any curve $F \subset \mathbf{C} P^{2}$ which can be embedded in a system $F_{t}$ of curves with $K_{n}$ as its limiting curve must have a complement with abelian fundamental group.

In [Sev 2], Severi claimed to have shown that any plane curve of degree $n$ with only nodes belongs to a system with limit $K_{n}$. Severi's proof is, however, erroneous and this is not known to be true.

Until recently one was thus left with:

ZARISKi ConJeCture. Let $C$ be a plane curve in $\mathbf{C} P^{2}$ with only node singularities. Then $\pi_{1}\left(C P^{2}-C\right)$ is abelian!

This past spring Fulton [F 1] using a powerful connectedness theorem proved by him and Hansen [FH] was able to show that if $C$ is an algebraic plane curve in $\mathbf{P}^{2}(k), k$ an algebraically closed field of arbitrary characteristic, whose only singularities are nodes then $\pi_{1}^{\text {alg }}\left(\mathbf{P}^{2}(k)-C\right)$ is abelian. By means of a beautiful modification of Fulton's argument Deligne [Del 1] (see also [F 2], [Del 2]) showed how a proof that $\pi_{1}\left(\mathbf{P}^{2}(\mathbf{C})-C\right)$ is abelian could be obtained. Thus Zariski's Conjecture is now a theorem.

We note that solving the problem of the fundamental groups of complements of plane curves would essentially solve it for all hypersurfaces in $\mathbf{C} \boldsymbol{P}^{n}$ since Zariski has shown [Zar 4]:

THEOREM 7.37 [Zar 4]. Let $V_{n-1}$ be an algebraic hypersurface in $\mathbf{C} P^{n}$. Then there exists an algebraically embedded image $H$ of $\mathbf{C P} P^{2}$ in $\mathbf{C P} P^{n}$ such that $\pi_{1}\left(\mathbf{C} P^{n}-V_{n-1}\right)=\pi_{1}\left(H-H \cap V_{n-1}\right)$.

As it is known that for any $d \leqslant \frac{1}{2}(n-1)(n-2)$ there exists an irreducible plane curve of degree $n$ with $d$-nodes which can be degenerated continuously to $K_{n}$, an alternative way to prove Zariski's conjecture for irreducible curves would have been to show that if $\Gamma_{1}, \Gamma_{2}$ are any two irreducible curves of degree $n$ in $\mathbf{C} P^{2}$ with $d$-nodes then there exists an autohomeomorphism $\phi$ of C $P^{2}$ with $\phi\left(\Gamma_{1}\right)=\Gamma_{2}$. (This is true if $n<3$.) However this seems quite difficult to do. If we allow our curves to have cusps as well then it is known that there exist two irreducible curves $\Gamma_{1}, \Gamma_{2}$ of degree six with six cusps such that $\pi_{1}\left(C P^{2}-\Gamma_{1}\right) \neq \pi_{1}\left(C P^{2}-\Gamma_{2}\right)$ [Zar 1]. Thus the placement problem for algebraic plane curves with node singularities is still open.

\section{AFTERWORD}

Having scattered various unsolved problems and plausible conjectures throughout the text of the article we refrain from repeating them now. For a very comprehensive collection of unsolved problems in low-dimensional topology we refer the reader to Kirby's exhaustive collection [Kirb 2]. The most pressing open problem of a more general sort is of course to find a 4-dimensional substitute for the Whitney lemma or means to avoid using it. 
The best current candidate for the former approach seems to be that of Casson [Cas 2], [Cas 3] and Freedman [Fr 2] while the best results obtained by avoiding any reliance on the Whitney lemma seem to be the ACD results of [MM 1], [MM 2], [Man 1], [Man 2], [Msh] and the fake $R P^{4}$ results of [CS 2]. New methods remain to be discovered and with them perhaps the key to a satisfactory understanding of what is happening in dimension 4. It is ironic that the worlds about which we know the least are our worlds: the 3-dimensional world of physical geometry and the 4-dimensional world of space-time. It is hoped that in the preceeding pages we have clarified that which is within the grasp of our knowledge and delineated that which remains to be understood.

\section{REFERENCES}

[Ax 1] J. W. Alexander, Note on Riemann spaces, Bull. Amer. Math. Soc. 26 (1920), 370-377.

[Ax 2] __, On the subdivision of a 3-space by a polyhedron, Proc. Nat. Acad. Sci. U.S.A. 10 (1924), 6-8.

[At 1] S. Akbulut, On 2-dimensional homology classes of 4-manifolds, Math. Proc. Cambridge Philos. Soc. 82 (1977), 99-110.

[At 2] 193-198. , On representing homology classes of 4-manifolds, Invent. Math. 49 (1978),

[At 3] __ Private communication.

[AK 1] S. Akbulut and R. Kirby, An exotic involution of $S^{4}$, Topology 18 (1979), 75-81.

[AK 2] __ Mazur manifolds, Michigan Math. J. (to appear).

[AK 3] _, Branched coverings of surfaces in 4-manifolds (preprint).

[AK 4] _ A simply connected 4-manifold with $b_{2}=22$ and $\sigma=16$ (preprint).

[AK 5] , Private communication.

[AF] A. Andreotti and T. Frankel, The 2nd Lefschetz Theorem on hyperplane sections, Global Analysis, Princeton Univ. Press, Princeton, N. J., 1969, pp. 1-20.

[AC] J. J. Andrews and M. L. Curtis, Knotted 2-spheres in the 4-sphere, Ann. of Math. (2) 70 (1959), 565-571.

[Bak] A. Bak, The computation of surgery groups of odd torsion groups, Bull. Amer. Math. Soc. 80 (1974), 1113-1116.

[Bd] D. Barden, $h$-cobordisms between 4-manifolds, Lecture notes, Cambridge Univ., 1964.

[BE] I. Berstein and L. Edmonds, The degree and branch set of a branched covering, Invent Math. 45 (1978), 213-220.

[Bord] J. M. Boardman, Some embeddings of 2-spheres in 4-manifolds, Proc. Cambridge Philos. Soc. 60 (1964), 354-356.

[BH] J. Boéchat and A. Haefliger, Plongements Differentiables des variétés orientes de dimension 4 dans $R^{7}$, Essays on Topology and Related Topics, Springer, New York, 1970, pp. 156-166.

[Bom] E. Bombieri, Canonical models of surfaces of general type, Publ. Math. Inst. Hautes Études Sci. 42 (1973), 447-495.

[Bo] W. W. Boone, The word problem, Ann. of Math. (2) 70 (1959), 207-265.

[BHP] W. W. Boone, W. Haken and V. Poenaru, On recursively unsolvable problems in topology and their classification, Cont. to. Math. Logic (Hanover Coll. 1966), pp. 37-74, North-Holland, Amsterdam, 1968.

[Br 1] William Browder, Surgery on simply-connected manifolds, Ergebnisse der Math., Band 65, Springer-Verlag, Berlin and New York, 1972.

[Br 2] __ Embedding smooth manifolds, Proc. Internat. Congr. Math., Moscow, 1966, Izdat. "Mir", 1968, pp. 712-719.

[Br 3] __ Manifolds and Homotopy Theory, Manifolds-Amsterdam 1970, Lecture Notes in Math., vol. 197, Springer-Verlag, Berlin and New York, 1971, pp. 17-35.

[Br 4] __, Diffeomorphisms of 1-connected manifolds, Trans. Amer. Math. Soc. 128 (1967), $155-163$.

[BLL] W. Browder, J. Levine and G. R. Livesay, Finding a boundary for an open manifold, Amer. J. Math. 87 (1965), 1017-1028. 
[Brwn] E. H. Brown, Abstract homotopy theory, Trans. Amer. Math. Soc. 119 (1968), 79-85.

[Bron] M. Brown, A proof of the generalized Schoenflies theorem, Bull. Amer. Math. Soc. 66 (1960), 74-76.

[Ca 1] S. S. Cairns, The manifold smoothing problem, Bull. Amer. Math. Soc. 67 (1961), 237-238.

[Ca 2] __ Introduction of a Riemannian geometry on a triangulable 4 manifold, Ann. of Math. (2) 45 (1944), 218-219.

[Ca 3] _ Homeomorphisms between topological manifolds and analytic manifolds, Ann. of Math. (2) 41 (1940), 796-808.

[Can] J. W. Cannon, Shrinking cell-like decompositions of manifolds: Codimension three, Ann. of Math. 110 (1979), 83-112.

[Cap 1] Sylvain E. Cappell, $A$ splitting theorem for manifolds, Invent. Math. 33 (1976), 69-170.

[Cap 2] ___, On connected sums of manifolds, Topology 13 (1974), 395-400.

[Cap 3] _ Superspinning and knot complements, Topology of Manifolds, Markham, Chicago, Ill., 1970, pp. 358-383.

[Cap 4] _ Manifolds with fundamental group a generalized free product. I, Bull. Amer. Math. Soc. 80 (1974), 1193-1198.

[Cap 5] _ Unitary nilpotent groups and hermitian K-theory, Bull. Amer. Math. Soc. 80 (1974), 1117-1122.

[CLS] S. Cappell, R. Lashof and J. Shaneson, A splitting theorem and the structure of 5-manifolds, Istituto Nazionale di Alta Matematica, Symposia Mathematica, Vol. 10, Academic Press, 1972, pp. 47-58.

[CS 1] S. Cappell and J. Shaneson, On four dimensional surgery and applications, Comment. Math. Helv. 46 (1971), 500-528.

[CS 2] __ Some new four-manifolds, Ann. of Math. (2) 104 (1976), 61-72.

[CS 3] __, Invariants of 3-manifolds, Bull. Amer. Math. Soc. 81 (1975), 559-562.

[CS 4] , Branched cyclic coverings, Ann. of Math. Studies, No. 84, Princeton Univ. Press, Princeton, N. J., 1975, pp. 165-173.

[CS 5] _ The codimension two placement problem and homology equivalent manifolds, Ann. of Math. (2) 99 (1974), 277-348.

[CS 6] __ Fundamental groups, $\Gamma$-groups, and codimension two sub-manifolds, Comment. Math. Helv. 51 (1976), 437-446.

[CS 7] ___ Submanifolds of codimension two and homology equivalent manifolds, Ann. Inst. Fourier (Grenoble) 23 (1973), 19-30.

[CS 8] __ Piecewise linear embeddings and their singularities, Ann. of Math. (2) 103 (1976), 163-228.

[CS 9] _ On the classification of singularities and immersions, Ann. of Math. (2) 103 (1976), 163-228.

[CS 10] __ Totally spineless manifolds, Illinois J. Math. 21 (1977), 231-239.

[CS 11] __ Embedding and immersion of four-dimensional manifolds in $\mathbf{R}^{6}$, Geometric Topology Proceedings of the 1977 Georgia Topology Conference, Academic Press, New York, 1979, pp. 301-305.

[CS 12] _, There exist inequivalent knots with the same complement, Ann. of Math. (2) 103 (1976), 349-353.

[CS 13] ___ Topological knots and knot cobordism, Topology 12 (1973), 33-40.

[Cas 1] A. J. Casson, Lectures on 4-manifolds (Lectures given at Cambridge University 1976), Private notes.

[Cas 2] __, Lecture at Institute for Advanced Study, 1976.

[Cas 3] _ Private communication.

[CG] A. J. Casson and C. McA. Gordon, On slice knots in dimension three, Proc. Sympos. Pure Math., vol. 32, Amer. Math. Soc., Providence, R. I., 1978, pp. 39-54.

[Cerf 1] J. Cerf, Sur les diffeomorphismes de la sphère de dimension trois $\left(\Gamma_{4}=0\right)$, Lecture Notes in Math., vol. 53, Springer-Verlag, Berlin and New York, 1968.

[Cerf 2] , Groupes d'automorphismes et groupes de difféomorphismes des variétés compactes de dimension 3, Bull. Soc. Math. France 87 (1959), 319-329.

[CR 1] P. E. Conner and F. Raymond, Deforming homotopy equivalences to homeomorphisms in aspherical manifolds, Bull. Amer. Math. Soc. 83 (1977), 36-85. 
[CR 2] , Actions of compact Lie groups on aspherical manifolds, Topology of Manifolds

(Proc. Inst., Univ. of Georgia, 1969), Markham, Chicago, Ill., 1970, pp. 227-264.

[CR 3] _ Holomorphic Seifert fiberings, Proc. Second Conf. on Compact Transformation Groups, Part II, Lecture Notes in Math., vol. 299, Springer-Verlag, Berlin and New York, 1972, pp. 109-123.

[Con] J. H. Conway, An enumeration of knots and links and some of their algebraic properties, Computational Problems in Abstract Algebra, Pergamon, Oxford, 1970, pp. 329-358.

[Crg] R. Craggs, Stable equivalence in and surgery presentations for 3-manifolds (preprint).

[Dehn] M. Dehn, Über die Topologie des dreidimensionalen Raumes, Math. Ann. 69 (1910), 137-168.

[Del 1] P. Deligne, Letter to W. Fulton, July 3, 1979.

[Del 2] ___ Le groupe fondamental du complement d'une courbe plane n'ayant que des points doubles ordinaires est abelian, Séminaire Bourbaki, November 1979.

[Durf] A. Durfee, 14 characterizations of rational double points (to appear).

[Ed 1] R. D. Edwards, The double suspension of a certain homology 3-spheres is $S^{5}$, Notices Amer. Math. Soc. 22 (1975), Abstract \# 75 T-G33.

[Ed 2] , Shrinking cell-like decompositions of manifolds (to appear).

[EK] J. Eells and N. H. Kuiper, An invariant for certain smooth manifolds, Ann. Mat. Pura Appl. (4) 60 (1962), 93-110.

[ENR] F. Enriques, Sulla construzione delle funzioni algebriche di due variabili . . . , Ann. Mat. Pura Appl. IV s. Vol. I. (1923), 185-198.

[EP] D. B. A. Epstein, Linking spheres, Proc. Cambridge Philos. Soc. 56 (1960), 215-219.

[Fenn] R. Fenn, On Dehn's lemma in 4 dimensions, Bull. London Math. Soc. 3 (1971), 79-81.

[FR] R. Fenn and C. Rourke, On Kirby's calculus of links, Topology 18 (1979), 1-15.

[Fin 1] R. Fintushel, Circle actions on simply-connected 4-manifolds, Trans. Amer. Math. Soc. 230 (1977), 147-171.

[Fin 2] __ Classification of circle actions on 4-manifolds (preprint).

[FP] R. Fintushel and S. P. Pao, Identification of certain 4-manifolds with group actions (preprint).

[Ford] L. Ford, Automorphic functions, 2nd ed., Chelsea, New York, 1951.

[Fox] R. H. Fox, A quick trip through knot theory, Topology of 3-Manifolds and Related Topics, Prentice-Hall, Englewood Cliffs, N. J., 1962, pp. 120-167.

[Frk] D. Franks, The signature Mod 8, Comment. Math. Helv. 48 (1973), 520-524.

[Fr 1] M. Freedman, Knot theory and 4-dimensional surgery (to appear).

[Fr 2] _ A fake $S^{3} \times R$, Ann. of Math. 110 (1979), 177-201.

[FK] M. Freedman and A. Kirby, Geometric proof of Rohlin's theorem, Proc. Sympos. Pure. Math. vol. 32, Amer. Math. Soc., Providence, R. I., 1978, pp. 85-97.

[F 1] W. Fulton, On the fundamental group of the complement of a node curve (preprint).

[F 2] _ Notes on connectivity in algebraic geometry (preprint).

[FH] W. Fulton and J. Hanson, A connected theorem for projective varieties with applications to intersections and singularities of mappings, Ann. of Math. 110 (1979), 159-166.

[GS 1] D. Galewski and R. Stern, Classification of simplicial triangulations of topological manifolds, Bull. Amer. Math. Soc. 82 (1976), 916-918.

[GS 2] (preprint). , Classification of simplicial triangulation of topological manifolds, Ann. of Math.

[GR] M. Gerstenhaber and O. Rothaus, The solution of sets of equations in groups, Proc. Nat. Acad. Sci. U.S.A. 48 (1962), 1531-1533.

[Glk] H. Gluck, The embeddings of 2-spheres in the 4-spheres, Bull. Amer. Math. Soc. 67 (1967), 586-589.

[Gor 1] C. McA. Gordon, Knots, homology spheres, and contractible 4-manifolds, Topology 14 (1975), 151-172.

[Gor 2] __ Knots in the 4-sphere, Comment. Math. Helv. 51 (1976), 585-596.

[Gor 3] _ Some higher-dimensional knots with the same homotopy groups, Quart. J. Math. Oxford Ser. (2) 24 (1973), 411-422.

[GM] L. Guillou and A. Marin, Une extension d'un théorème de Rohlin sur la signature, C. R. Acad. Sci. Paris Sér A-B 285 (1977), A95-A98.

[Har] J. Harer, Hypersurfaces in $\mathrm{C}^{3}$ and $\mathrm{CP}^{3}$, Math. Ann. 238 (1978), 51-58.

[HC] J. Harer and A. Casson (to appear). 
[HKK] J. Harer, A. Kas and R. C. Kirby (to appear).

[Hemp] J. Hempel, 3-manifolds, Ann. of Math. Studies No. 86, Princeton Univ. Press, Princeton, N.J.; Univ. of Tokyo Press, Tokyo, 1976.

[Hd] A. Hilden, Every closed orientable 3-manifold is a 3-fold branched covering space of $S^{3}$, Bull. Amer. Math. Soc. 80 (1974), 1243-1244.

[Hi 1] M. W. Hirsch, Obstruction theories for smoothing manifolds and maps, Bull. Amer. Math. Soc. 69 (1963), 352-356.

[Hi 2] , On combinatorial submanifolds of differentiable manifolds, Comment. Math. Helv. 36 (1961), 103-111.

[Hi 3] __ PL embedding $M^{4}$ into $R^{7}$, Proc. Cambridge Philos. Soc. 61 (1965), 657-658.

[Hi 4] __ On embedding differentiable manifolds in euclidean space, Ann. of Math. (2) 73 (1961), 566-571.

[Hi 5] __ Immersions of manifolds, Trans. Amer. Math. Soc. 93 (1959), 242-276.

[HM] M. W. Hirsch and B. Mazur, Smoothings of piecewise linear manifolds, Ann. of Math. Studies, No. 80, Princeton Univ. Press, Princeton, N. J.; Tokyo Univ. Press, Tokyo, 1974.

[Hirz 1] F. Hirzebruch, Topological methods in algebraic geometry, Springer-Verlag, New York, 1966.

[Hirz 2] F. Hirzebruch, W. D. Neumann and S. S. Koh, Differentiable manifolds and quadratic forms, Marcel Dekker, New York, 1971.

[HS 1] W. Hsiang and J. L. Shaneson, Fake tori, Topology of Manifolds, Markham, Chicago, Ill., 1970, pp. 18-51.

[HS 2] _ Fake tori, the annulus conjecture, and the conjectures of Kirby, Proc. Nat. Acad. Sci. U.S.A. 62 (1969), 687-691.

[HSZ] W. Hsiang and R. Szczarba, On embedding surfaces in four manifolds, Proc. Sympos. Pure Math., vol. 22, Amer. Math. Soc., Providence, R. I., 1970, pp. 97-103.

[Hus] D. Husemoller, Fiber bundles, McGraw-Hill, New York, 1966.

[JS] W. Jaco and P. Shalen, Seifert fibered spaces in 3-manifolds, Mem. Amer. Math. Soc. 220, 1979.

[Kap] S. J. Kaplan, Constructing framed 4-manifolds with given almost framed boundaries, Trans. Amer. Math., Soc. 254 (1979), 237-263.

[Kas 1] A. Kas, Weierstrass normal forms and invariants of elliptic surfaces, Trans. Amer. Math. Soc. 225 (1977), 259-266.

[Kas 2] _ On deformation types of regular elliptic surfaces, Complex Analysis and Algebraic Geometry, Cambridge Univ. Press, New York and London, 1977, pp. 107-112.

[Kato] M. Kato, A concordance classification of PL homeomorphisms of $S^{p} \times S^{q}$, Topology 8 (1969), 371-383.

[KM 1] M. Kervaire and J. W. Milnor, On 2-spheres in 4-manifolds, Proc. Nat. Acad. Sci. U.S.A. 47 (1961), 1651-1657.

[KM 2] __ Groups of homotopy spheres. I, Ann. of Math. (2) 77 (1963), 504-537.

[Kirb 1] R. C. Kirby, A calculus for framed links in $S^{3}$, Invent. Math. 45 (1978), 35-56.

[Kirb 2] _ Problems in low dimensional manifold theory, Proc. Sympos. Pure Math., vol. 32, Amer. Math. Soc., Providence, R. I., 1978, pp. 273-312.

[Kirb 3] __ Some conjectures about four-manifolds, Actes Congrès Internat. Math., 1970, Tome 2, pp. 79-84.

[Kirb 4] (1963), 504-537.

[Kirb 5] _ Private notes.

[KS 1] R. C. Kirby and L. C. Siebenmann, Foundational essays on topological manifolds, smoothing and triangulation, Princeton Univ. Press, Princeton, N. J., 1977.

[KS 2] __ Some theorems on topological manifolds, Manifolds-Amsterdam 1970, Lecture Notes in Math., vol. 197, Springer-Verlag, Berlin and New York, 1971, pp. 1-7.

[Kob] K. Kobayashi, On a homotopy version of the 4-dimensional Whitney lemma, Math. Sem. Notes Kobe Univ. 5 (1977), 109-116.

[K 1] K. Kodaira, On compact analytic surfaces. I, II, III, Ann. of Math. (2) 71 (1960), 111-152; 77 (1963), 563-626; 78 (1963), 1-40.

[K 2] _ On the structure of compact complex analytic surfaces. I, II, IV, Amer. J. Math. 86 (1964), 781-798; 88 (1966), 682-721; 90 (1968), 1048-1066. 
[K 3] , On homotopy $\mathrm{K} 3$ surfaces, Essays on Topology and Related Topics, Springer, New York, 1970, pp. 58-69.

[Lg] S. Lang, Introduction to differentiable manifolds, Interscience, New York, 1962.

[Lash] R. Lashof, The immersion approach to triangulation and smoothing, Proc. Adv. Study Inst. Alg. Top. Aarhus 1970, 282-355.

[LR] R. Lashof and M. Rothenberg, Triangulation of manifolds. I, II, Bull. Amer. Math. Soc. 75 (1969), 750-757.

[LR 2] _ Microbundles and smoothing, Topology 3 (1965), 357-388.

[LS] R. Lashof and J. Shaneson, Smoothing four-manifolds, Invent. Math. 14 (1971), 197-210.

[LS 2] 171-175.

[LP] F. Laudenbach and V. Penaru, A note on 4-dimensional handlebodies, Bull. Soc. Math. France 100 (1972), 337-347.

[L] H. Laufer, Normal two dimensional singularities, Ann. of Math. Studies no. 61, Princeton Univ. Press, Princeton, N. J., 1968.

[Law 1] T. Lawson, Trivializing h-cobordisms by stabilization, Math. Z. 156 (1977), 211-215.

[Law 2] (preprint).

[Law 3] __ Decomposing 5-manifolds as doubles, Tulane, Univ., 1977 (preprint).

[Lees] J. Alexander Lees, The surgery obstruction groups of C.T.C. Wall, Advances in Math. 11 (1973), 113-156.

[Lef] S. Lefschetz, $L$ analysis situs et la géometrie algèbrique, Paris, 1924.

[Lick] W. B. R. Lickorisch, A representation of orientable combinatorial 3-manifolds, Ann. of Math. (2) 76 (1962), 531-540.

[Lom] S. J. Lomonaco, The homotopy groups of knots. I, II (to appear).

[McM] D. R. McMillan, A criterion for cellularity in a manifold, Ann. of Math. (2) 79 (1964), 327-337.

[Mah] M. Mahowald, The order of the image of the J-homomorphism, Bull. Amer. Math. Soc. 76 (1970), 1310-1313.

[Man 1] R. Mandelbaum, On irrational connected sums, Trans. Amer. Math. Soc. 247 (1979), 137-156.

[Man 2] —, Studies in algebraic topology, Advances in Math. Supplem. Studies vol. 5 (1979), 143-166.

[Man 3] __ On special handlebody decompasitions, Proc. Amer. Math. Soc. (to appear).

[Man 4] _ Decomposing analytic surfaces, Geometric Topology Proceedings of the 1977 Georgia Topology Conference, Academic Press 1979, pp. 147-218.

[Man 5] _ (to appear).

[MM 1] R. Mandelbaum and B. Moislezon, On the topological structure of non-singular algebraic surfaces in $\mathrm{CP}^{3}$, Topology 15 (1976), 23-40.

[MM 2] __, On the topology of algebraic surfaces, Trans. Amer. Math. Soc. (to appear).

[MM 3] _ _ Numerical link invariants (preprint).

[Mas] W. S. Massey, Proof of a conjecture of Whitney, Pacific J. Math. 31 (1969), 143-156.

[Mt 1] Y. Matsumoto, An elementary proof of Rohlin's Signature Theorem and its extension by Guillou and Marin, Notes, IAS Geometric Topology Seminar.

[Mt 2] __, A 4-manifold which admits no spine, Bull. Amer. Math. Soc. 81 (1975), 467-470.

[Mt 3] _ Some wild embeddings of $S^{1} \times S^{m+1}$ into $R^{m+4}$ (to appear).

[Mt 4] - Secondary intersectional properties of 4-manifolds and Whitney's trick, Proc. Sympos. Pure Math., vol. 32 Amer. Math. Soc., Providence, R. I., 1978, pp. 99-110.

[Mt V] Y. Matsumoto and G. A. Venema, Failure of the Dehn lemma on contractible 4-manifolds, Invent. Math. 51 (1979), 205-218.

[Mat] T. Matumoto, Variétés simpliciales d'homologie et variétés topologiques metrisable, Thèse Univ. de Paris-Sud Orsay, 1976.

[Mz] B. Mazur, A note on some contractible 4-manifolds, Ann. of Math. (2) 73 (1961), 221-228.

[M 1] J. Milnor, On simply-connected 4-manifolds, Symp. Internat. de Top. Alg. (Mexico 1956) Mexico, 1958, pp. 122-128.

[M 2] _ Differentiable manifolds which are homotopy spheres (Unpublished Notes, Princeton, 1959). 
[M 3] _ A procedure for killing the homotopy groups of differentiable manifolds, Proc.

Sympos. Pure Math., vol. 3, Amer. Math. Soc., Providence, R. I., 1961, pp. 39-55.

[M 4] __ Lectures on the $h$-cobordism theorem, Princeton Mathematical Notes, 1965.

[M 5] _ Whitehead torsion, Bull. Amer. Math. Soc. 72 (1966), 358-426.

[MH] J. Milnor and J. Husemoller, Symmetric bilinear forms, Ergebnisse der Mathematik und ihrer Grenzgebiete, Band 73, Springer-Verlag, Berlin and New York, 1973, pp. 1-147.

[MK] J. Milnor and M. Kervaire, Bernoulli numbers, homotopy groups and a Theorem of Rohlin, Proc. Internat. Congr. Math. Edinburgh, 1958, pp. 454-458.

[MS] J. Milnor and J. D. Stasheff, Characteristic classes, Ann. of Math. Studies no. 76, Princeton Univ. Press, Princeton, N. J., 1974.

[My] Y. Miyaoka, On the Chern numbers of surfaces of general type, Invent. Mathematicae $\mathbf{4 2}$ (1977), 225-237.

[Moise] E. Moise, Affine structures on 3-manifolds, Ann. of Math. (2) 56 (1952), 96-114.

[MSb] T. Matumoto and L. Siebenmann, The topological s-cobordism theorem fails in dimension 4 or 5, Math. Proc. Cambridge. Philos. Soc. 84 (1978), 85-87.

[Msh] B. Moishezon, Complex surfaces and connected sums of complex projective planes, Lecture Notes in Math., vol. 603, Springer-Verlag, Berlin and New York, 1977.

[Mont 1] J. M. Montesinos, A representation of closed-orientable 3-manifolds as 3-fold branched coverings of $S^{3}$, Bull. Amer. Math. Soc. 80 (1974), 845-846.

[Mont 2] _, 4-manifolds, 3-fold covering spaces and ribbons, Trans. Amer. Math. Soc. 245 (1978), 453-467.

[Mont 3] __ Heegard diagrams for closed 4-manifolds, Geometric Topology, Proceedings of the 1977 Georgia Topology Conference, Academic Press, 1979, pp. 219-238.

[Mont 4] _ 3 manifolds as 3-fold branched coverings of $S^{3}$, Quart. J. Math. Oxford Ser. (2) 27 (1976), 85-97.

[Most] G. D. Mostow, Strong rigidity of locally symmetric spaces, Ann. of Math. Studies, no. 78, Princeton Univ. Press, Princeton, N. J., 1973.

[Mun 1] J. Munkres, Elementary differential topology, Ann. of Math. Studies no. 54 Princeton Univ. Press, Princeton, N. J., 1966.

[Mun 2] __ Concordance is equivalent to smoothability, Topology 5 (1966), 371-389.

[Nor] R. A. Norman, Dehn's Lemma for certain 4-manifolds, Invent. Math. 7 (1969), 143-147.

[N] S. P. Novikov, Homotopically equivalent smooth manifolds. I (in Russian), Izv. Akad. Nauk. SSR Ser. Mat. 28 (2) (1964), 265-374; English Transl., Amer. Math. Soc. Transl. (2) no. 48 (1965), 271-396.

[Orl] P. Orlik, Seifert manifolds, Lecture Notes in Math., vol. 291, Springer-Verlag, Berlin and New York, 1972.

[OR 1] P. Orlik and F. Raymond, Actions of the torus on 4-manifolds. I, Trans. Amer. Math. Soc. 152 (1970), 531-559.

[OR 2] __, Actions of the torus on 4-manifolds. II, Topology 13 (1974), 89-112.

[Papa 1] C. D. Papakyriakopoulos, On Dehn's lemma and the asphericity of knots, Ann. of Math. (2) 66 (1957), 1-26.

[Papa 2] ___ On solid tori, Proc. London Math. Soc. 7 (1957), 281-299.

[Pao 1] P. Pao, The topological structure of 4-manifolds with effective torus action. I, Trans. Amer. Math. Soc. 227 (1977), 279-317.

[Pao 2] _ The topological structure of 4-manifolds with effective torus action. II, Illinois J. Math. 21 (1977), 883-894.

[Pit] H. Pittie, Complex and almost complex manifolds, Complex Analysis and its Applications, Vol. III, Internat. Atomic Energy Agency, Vienna, 1976, pp. 121-131.

[Pon] L. S. Pontryagin, Smooth manifolds and their applications in homotopy theory, Amer. Math. Soc. Transl. (2) no. 11 (1959), 1-114.

[Q] F. Quinn, $A$ stable Whitney trick in dimension four (to appear).

[Ram] C. P. Ramanujam, A topological characterization of the affine plane as an algebraic variety, Ann. of Math. (2) 94 (1971), 69-88.

[Ran] A. Ranicki, On algebraic L-theory. Parts I, II, Proc. London Math. Soc. 27 (1973), pp. 101-125; 126-158; Part III, Lecture Notes in Math., vol. 343, Springer-Verlag, Berlin and New York, 1973.

[Reid] M. Reid, Article On Bogomolov's Theorem (preprint). 
[RIf] D. Rolfsen, Knots and links, Lecture Notes, Univ. of British Columbia, Vancouver, 1975.

[R 1] V. A. Rohlin, New results in the theory of 4-dimensional manifolds, Dokl. Akad. Nauk SSSR 84 (1952), 221-224. (Russian)

[R 2] _ Two-dimensional submanifolds of four-dimensional manifolds, Funktional. anal. i

Priložen. 5 (1971), 48-60 (Russian); English transl., Functional Anal. Appl. 5 (1971), 39-48.

[R 3] __ Proof of Gudkov's hypothesis, Funkcional. anal. i Priložen. 6 (1972), 62-64 (Russian); English Transl., Functional Anal. Appl. 6 (1972), 136-138.

[RS] C. P. Rourke and B. J. Sanderson, Introduction to piecewise linear topology, Ergebnisse der Mathematik und ihrer Grenzgebiete, Band 69, Springer-Verlag, Berlin and New York, 1972.

[Rb] J. H. Rubinstein, Private correspondence.

[Rd] L. Rudolph, $A$ Morse function for a surface in $\mathrm{CP}^{3}$, Topology 14 (1975), 301-303.

[Sá] E. C. de Sá, A link calculus for 4-manifolds, Topology of Low-Dimensional Manifolds, Lecture Notes in Math., vol. 722, Springer-Verlag, Berlin and New York, 1979, pp. 16-31.

[Sch 1] M. Scharlemann, Constructing strange manifolds with the dodecahedral space, Duke Math. J. 43 (1976), 33-40.

[Sch 2] __, Equivalence of 5-dimensional S-cobordisms, Proc. Amer. Math. Soc. 53 (1975), 508-510.

[Sch 3] __ Transversality theories at dimension 4, Invent. Math. 33 (1976), 1-14.

[Sf] H. Seifert, Topologie dreidimensionaler gefaserter Räume, Acta. Math. 60 (1933), 147-238.

[Ser 1] J. P. Serre, Cours d'arithmetique, Presses Universitaires de France.

[Ser 2] __ Formes bilinéaires symétriques entières à discriminant \pm 1 , Séminaire Henri Cartan 14e Année (Topologie differentielle), 1961/62, exp. 14-15.

[Sev 1] Francesco Severi, Intorno ai punti doppi improprî di una superficie generale dello spazio a quattro dimensioni, e a' suoi punti tripli apparenti, Rend. Circ. Mat. Palermo 15 (1901), 33-51; correction, ibid., p. 160.

[Sev 2] - Sulla deficienza della serie caratteristica di un sistema lineare di curve appartenenti ad una superficie algebrica, Atti. Reale Accad. Lincei Rend. (5) 12 (1903), no. 2, 250-257.

[Shaf 1] I. R. Shafarevitch, Basic algebraic geometry, Springer-Verlag, Berlin and New York, 1975.

[Shaf 2] _ Algebraic surfaces, Proc. Steklov Inst. Math. 75 (1965), Amer. Math. Soc., Providence, R. I., 1967.

[Sh 1] J. L. Shaneson, Non-Simply connected surgery and some results in low dimensional topology, Comment. Math. Helv. 45 (1970), 333-352.

[Sh 2] __, On non-simply connected manifolds, Proc. Sympos. Pure Math., vol. 22, Amer. Math. Soc., Providence, R. I., 1970, pp. 221-229.

[Sh 3] _ Wall's surgery obstruction groups for $G \times Z$, Ann. of Math. (2) 90 (1969), 296-334.

[Sh 4] _ Hermitian K-theory in Topology, Lecture Notes in Math., vol. 343, "Hermitian K-Theory and geometric applications" 1-41 Springer (1973).

[Sb 1] L. C. Siebenmann, Disruption of low-dimensional handlebody theory by Rohlin's Theorem, Topology of Manifolds, Markham, Ill., 1970, pp. 57-76.

[Sb 2] __ Are nontriangulable manifolds triangulable? Topology of Manifolds, Markham, III., 1970, pp. 77-84.

[Sb 3] _ Topological manifolds, Proceedings Internat. Congr. Mathematics, Nice (1970), vol. 2, Gauthier-Villars, 1971, pp. 143-163.

[Sb 4] __ Lecture at the Institute of Advanced Study, 1977.

[Sb 5] __ Amorces de la chirugie en dimension quatre: Un $S^{3} \times R$ exotique, [d'après A. H.

Casson and M. H. Freedman] Séminaire Bourbaki 1978/79 Feb. 1979, pp. 536-01 to 536-25.

[Sm] S. Smale, Generalized Poincaré's conjecture in dimensions greater than 4, Ann. of Math. (2) 74 (1961), 391-406.

[Spr 1] E. H. Spanier, Algebraic topology, McGraw-Hill, New York, 1966.

[Spr 2] —_, On the homology of Kummer manifolds, Proc. Amer. Math. Soc. 7 (1956), $155-160$.

[St 1] J. R. Stallings, Notes on polyhedral topology, Tata Institute of Fundamental Research, Bombay, 1968.

[St 2] __, On the loop theorem, Ann. of Math. (2) 72 (1960), 12-19. 
[St 3] Conn., 1972. , Group theory and three dimensional manifold, Yale Univ. Press, New Haven,

[Stn] N. Steenrod, The topology of fibre bundles, Princeton Univ. Press, Princeton, N. J., 1951.

[S 1] D. Sullivan, Thesis, Princeton, 1965.

[S 2] , Triangulating and smoothing homotopy equivalences and homeomorphisms, Geometric Topology Seminar Notes, Princeton Univ. 1967.

[Thm] R. Thom, Quelques propriétés globales des variétés differentiables, Comment. Math. Helv. 28 (1954), 17-86.

[Tr] A. G. Tristram, Some cobordism invariants for links, Proc. Cambridge Philos. Soc. 66 (1969), 251-264.

[Wald] F. Waldhausen, On irreducible 3-manifolds which are sufficiently large, Ann. of Math. (2) 87 (1968), 56-88.

[Wa 1] C. T. C. Wall, Diffeomorphisms of 4-manifolds, J. London Math. Soc. 39 (1964), 131-140.

[Wa 2] , On simply-connected 4-manifolds, J. London Math. Soc. 39 (1964), 141-149.

[Wa 3] , Surgery on compact manifolds, Academic Press, London and New York, 1970.

[Wa 4] , Foundations of algebraic $L$-theory, Algebraic $K$-theory. III, Lecture Notes in Math., vol. 343, Springer-Verlag, Berlin and New York, 266-300.

[Wa 5] _ On homotopy tori and the annulus theorem, Bull. London Math. Soc. 1 (1969), 95-97.

[Wa 6] __ Locally flat PL submanifolds with codimension two, Proc. Cambridge Philos. Soc. 63 (1967), 5-8.

[Wa 7] Some L groups of finite groups, Bull. Amer. Math. Soc. 39 (1973), 526-529.

[Wa 8] __ On the orthogonal groups of unimodular quadratic forms, Math. Ann. 147 (1962), 328-338.

[Wlc] A. Wallace, Homology theory on algebraic varieties, Pergamon Press, New York, 1958.

[W 1] J. H. C. Whitehead, A certain open manifold whose group is unity, Quart. J. Math. 6 (1935), 268-279.

[W 2] __, On simply connected 4-dimensional polyhedra, Comment. Math. Helv. 22 (1949), 48-92.

[Whit 1] H. Whitney, The self-intersection of a smooth n-manifold in $2 n$-space, Ann. of Math. (2) 45 (1944), 220-246.

[Whit 2] H. Whitney, On the topology of differentiable manifolds, Lectures in Topology, Michigan Univ. Press, Ann Arbor, Mich., 1940.

[Y] S. T. Yau, Calabi's conjecture and some new results in algebraic geometry, Proc. Nat. Acad. Sci. U.S.A. 74 (1977), 1798-1799.

[Zar 1] O. Zariski, An introduction to the theory of algebraic surfaces, (2nd ed.), Lecture Notes in Math., vol. 83, Springer-Verlag, Berlin and New York, 1972.

[Zar 2] __ Introduction to the problem of minimal models in the theory of algebraic surfaces, Publ. Math. Soc. Japan, no. 4, Math. Soc. Japan, Tokyo, 1958.

[Zar 3] __, On the problem of existence of algebraic function of two variables, . . , Amer. J. Math. 51 (1929), 305-328.

[Zar 4] , A theorem on the Poincaré group of an algebraic hypersurface, Ann. of Math. (2) 38 (1937), 131-141.

[Z 1] E. C. Zeeman, Seminar on combinatorial topology, Mimeo, I.H.E.S., Bures-Sur-Yvette and Univ. of Warwick, 1963.

[Z 2] The Poincaré conjecture for $N>5$, Topology of 3-manifolds, Prentice-Hall, Englewood Cliffs, N. J., 1962, pp. 198-203.

[Z 3] __, On the dunce hat, Topology 2 (1964), 341-358.

[Z 4] __, Twisting spun knots, Trans. Amer. Math. Soc. 115 (1965), 471-495.

Department of Mathematics, University OF Rochester, Rochester, New YoRk 14627 
